\title{
Kapitel 6 \\ Die Ausdrucks-Seite narrativer Vertextungen bei Paulus
}

\section{Lexikalisches Aktionsartpotenzial}

\subsection{Verbklassen und Aktionsart in GGNT}

Wie bereits erläutert, besteht der Aussage-Kern einer Proposition in der Regel aus einem Vorgangskonzept (z. B. ,reden'), kann aber auch durch ein Beziehungskonzept (z. B. ,glauben') oder ein Zustandskonzept (z. B. ,sein') vertreten sein. Für das Verständnis des Erzählten ist eine möglichst präzise Erfassung dieses Konzepts von elementarer Bedeutung. Die Rede von der Handlung einer Erzählung setzt voraus, dass die einzelnen Elemente des Plots identifiziert und klassifiziert werden können. Es ist daher von grundsätzlicher Bedeutung für die narratologische Analyse, die Aussage-Kerne der Propositionen, aus welchen sich eine Erzählung inhaltlich zusammensetzt, korrekt zu beschreiben.

Auf der Ebene der Grammatik-Seite des Textes werden diese Konzepte typischerweise durch Verben ausgedrückt, welche die syntaktische Rolle des Prädikats innehaben. ${ }^{1}$ Den verschiedenen Propositionskernen entsprechen dabei unterschiedliche Klassen an Verben, wobei die Unterteilung der jeweiligen Verbsemantik folgt. ${ }^{2}$ Heinrich von Siebenthal differenziert zwischen folgenden Verbklassen, die sich wohl durch die in der Abb. 27 ergänzten linguistischen Kriterien differenzieren lassen:

\begin{tabular}{|c|c|c|c|c|}
\hline \multicolumn{2}{|c|}{ Verbsemantik } & \multirow[t]{2}{*}{ Beispiel } & \multicolumn{2}{|c|}{ Kriterien } \\
\hline & & & Durativität & Agentivität \\
\hline \multicolumn{2}{|l|}{ Zustände } & 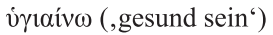 & + & - \\
\hline \multirow[t]{3}{*}{ Geschehnisse } & Ereignisse & $\pi i ́ \tau \tau \omega$ (,fallen') & - & - \\
\hline & Vorgänge & 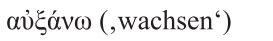 & + & - \\
\hline & Handlungen & 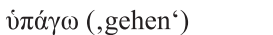 & + & + \\
\hline
\end{tabular}

Abb. 27: Unterteilung der griechischen Verben nach Heinrich von Siebenthal mit ergänzten Unterscheidungskriterien. Zustände und Geschehnisse lassen sich durch das Kriterium der Stativität unterscheiden. Es ist für die trennscharfe Differenzierung zwischen den Unterkategorien jedoch nicht notwendig.

1 Vgl. GGNT 22f.

2 GGNT $22 \mathrm{f}$. 
Zwei Beobachtungen können hierzu angestellt werden: Erstens ist es aus narratologischer Perspektive ein grundsätzliches Problem an der Übersicht von Heinrich von Siebenthal, dass der Terminus „Ereignis“ für ein recht spezifisches Phänomen verwendet wird, während die hier zugrundegelegte Ereignis-Konzeption so weit gefasst ist, dass sie sogar Geschehnisse und Zustände umfasst. Es sollen daher im Rest des Abschnitts noch einige andere Möglichkeiten bedacht werden, wie Aktionsarten differenziert werden können. Gerade auch für die später (Abschnitt 2) erfolgende Hinzunahme des Aspekts ist dies eine notwendige Grundlage.

Zweitens ist auffallend, dass Heinrich von Siebenthal an dieser Stelle nicht den Terminus „Aktionsart“ für die Verbsemantik verwendet noch auf den entsprechenden Paragraphen verweist, während andererseits die dort gebotene Definition offensichtlich eine Nähe der Konzeption zu den oben gelisteten Verbklassen aufweist: ${ }^{3}$

Unter Aktionsart versteht man die Art, wie der (durch das Lexikon/Wörterbuch standardmäßig zugeordnete) Verbinhalt normalerweise verwirklicht wird, und zwar natürlicherweise, unabhängig von der subjektiven Betrachtungsweise der Sprechers/Schreibers, als fester Teil der (lexikalischen) Bedeutung des jeweiligen Verbs.

Dieser Zusammenhang zwischen dem vom Sprecher gewählten Lexem und der „Aktionsart“ soll im Folgenden genauer diskutiert werden.

\subsection{Aktionsart als Situationstyp}

Heinrich von Siebenthal spricht im Hinblick auf die Aktionsart vom „Verbinhalt.“ Genauer genommen ist das Prädikat mitsamt seinen Ergänzungen - also der Prädikatsverband ${ }^{4}$ - für die Beurteilung der Aktionsart relevant, da Verben häufig mit mehreren Satzmustern/Satzplänen verbunden sind und die Aktionsart dabei variieren ${ }^{5}$ kann. „Christoph rannte einen Kilometer“ beinhaltet gegenüber „Christoph rannte“ beispielsweise einen Endpunkt. ${ }^{6}$

3 GGNT 194m.

4 Siehe zu den verschiedenen hier aufgerufenen Konzepten GGNT 254c.

5 Variation kann auch durch nicht-obligatorische Elemente erfolgen. Beispielsweise bringen die Sätze „Bill knew the truth.“ und „Suddenly Bill knew the truth.“ unterschiedliche Situationstypen zum Ausdruck („State“ vs. „Achievement“). Smith, Parameter, 8-9 unterscheidet hier zwischen einem „basic-level“ Situationstyp, welcher der Verbkonstellation immer zur Verfügung steht, und einem abgeleiteten Situationstyp, welcher durch zusätzliche Information (Adverbien etc.) aktiviert werden muss. Vgl. auch S. 48-53.

6 Siehe Thomson, „Aspect,“ 30. Horrocks, „Envoi,“ 629 differenziert zwischen verschiedenen Beschreibungsebenen: Telizität sei eine Eigenschaft des Prädikatsverbandes (und der dadurch 
Da im Rahmen der konkreten Äußerung unter anderem durch die Wahl des Aspekts $^{7}$ eine Modifikation des Gemeinten erfolgt (siehe unten, Abschnitt 2.3), ist es sinnvoll, in der Notation den rein lexikalisch-semantischen Beitrag zum Kommunikat abzuheben. Smith schreibt etwa Subjekt und Prädikatsverband als „Verbkonstellation“ in eckigen Klammern, also [Christoph rennen] bzw. [Christoph rennen einen Kilometer]. ${ }^{8}$ Davon $\mathrm{zu}$ unterscheiden sei dann nochmals das dadurch (bei Smith in geschweiften Klammern markierte) ausgedrückte Konzept \{Christoph rennen\} bzw. \{Christoph rennen einen Kilometer\}, welches durch die Verbkonstellation vermittelt wird und im konkreten Satz mit Zeitbezug erscheint. ${ }^{9}$

Zu beachten ist, dass in der Literatur eckige Klammern analog zu Smiths geschweiften Klammern auch für die deskriptive Bedeutung von Sätzen verwendet werden (Propositionen; siehe oben, Kapitel 4, Abschnitt 5.1) werden. Sowohl als Proposition als auch als Verbkonstellation kann die Notation [Christoph rennen] auch für die Sätze „Rennt Christoph?“ und „Christoph, renne!“ stehen. [Christoph rennen] in der propositionalen (konzeptuellen) Verwendung von [Christoph

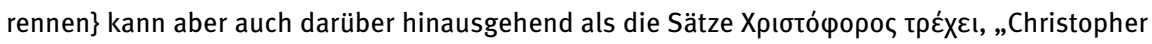
runs“ oder eventuell auch „Christoph joggt“ erscheinen. Im Hinblick auf die Verbkonstellation

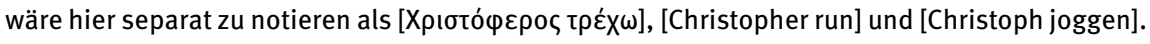

Bei den vermittelten Konzepten (d. h. $\{$... $\}$ bei Smith) handelt es sich ganz allgemein um „Situationen,“ welche (unter anderem) ${ }^{10}$ im Hinblick auf ihre temporale

bezeichneten Situationen), im Hinblick auf Aktionsart im Sinn des lexikalisch-semantischen Beitrags des Verbs könne man hingegen nur „terminative vs. nonterminative“ gegenüberstellen: „Thus ,wash“ in isolation denotes a nonterminative activity, but ,washes cars for a living، is atelic and ,washes the cars in half an hour' is telic.“

7 Mit „Aktionsart“ bezeichnen einige ältere Grammatiken (wie etwa BDR) das, was hier als „Aspekt“ bezeichnet wird und im nächsten Abschnitt (Abschnitt 2) besprochen wird. Vgl. zur Terminologie GGNT 192a. Thomson, „Aspect,“ 28-31 diskutiert sechs verschiedene Konzepte, die in der Forschung mit dem Ausdruck „Aktionsart“ (auch in der englischsprachigen Literatur) verbunden sind.

8 So kann im Englischen die Verbkonstellation [The ship move] als Aktivität („The ship moved.“) oder Zustand („The ship was moving.“) dargestellt werden. Siehe Smith, Paramter, 7. Aufgegriffen von Thomson, „Aspect,“ 48. Vgl. auch die ähnliche Praxis bei Crellin, „Semantics,“ 435.

9 Smith, Parameter, 17: ,The situation type of a sentence is conveyed by the verb and its arguments, the verb constellation.“

10 Vgl. Smith, Parameter, 6. Thomson, „Aspect,“ 49 spricht vom „procedural character“ der Situationstypen und bevorzugt die Bezeichnung als „procedural class,“ was in der Tat gut zum Ausdruck bringt, dass die Typisierung sich spezifisch auf ,sets of temporal characteristics communicated by the verb constellation“ bezieht. 
Konfiguration verschiedenen Typen zugeordnet werden können. ${ }^{11}$ Nochmals zu unterscheiden von der konzeptuellen Ebene sind die Situationen, wie sie sich in der realen oder erzählten fiktiven Welt ereignen. ${ }^{12}$ Wir sprechen dann von „Sachverhalten“13 bzw. schlicht von „tatsächlichen Situationen“ der realen oder fiktiven Welt der Erzählung.

Die Rede von z. B. „punktuellen tatsächlichen“ Ereignissen setzt allerdings bereits die Bewertung innerhalb eines psychologischen Systems voraus. Der Sachverhalt wird also bereits durch die Wahrnehmung und das Verständnis des Sprechers aufgegriffen. Streng genommen bezieht sich die Charakterisierung einer „Aktionsart“ beziehungsweise ,actionality“14 also auf eine Beschreibungsebene, bei welcher die konzeptuellen Referenten eines sprachlichen Ausdrucks im Zentrum stehen. ${ }^{15}$

Auf jeden Fall nur mit einer Qualifikation zu verstehen ist die Rede von „punktuellen“ Verben etc., die hier der Einfachheit halber teilweise beibehalten wird: Verben bzw. auch Verbkonstellationen verfügen streng genommen lediglich über ein „aktionales Potenzial.“16 In der Lexikalisierung schlägt sich die Typologisierung von Situationen durch eine Sprechergemeinschaft nieder. Die Wahl eines bestimmten Lexems kann daher als erster Anhaltspunkt dafür angesehen werden, welche Aktionsart (welcher Situationstyp) dargestellt werden soll. Die entsprechenden Eigenschaften kommen letztlich aber den konzeptualisierten Sachverhalten zu. Entsprechend ist der in diesem Abschnitt analysierte Beitrag zu Erzählungen auch unter der (in der exegetischen Literatur recht unüblichen, letztlich aber sehr treffenden) Überschrift des „lexikalischen Aktionsartpotenzials“ diskutiert. Der Fokus liegt auf dem Gebrauch bestimmter Lexeme, das dabei verfolgte Interesse hat jedoch die - unter anderem durch die Lexik - kommunizierten Situation(styp)en im Blick.

11 Siehe etwa Bache, Study, 119. Vgl. zu „Situation“ als „catchall for actions, events, activities, and states“ (Thomson, „Aspect,“ 45). Die in der erzähltheoretischen Literatur vorgenommenen Klassifikationen sind vielfältig und knüpfen nicht immer an die hier herangezogenen grundlegenden linguistischen Studien an. Siehe etwa Herman, „Event and Event-Types,“ 151-152 ohne Nennung von Vendler, „Verbs and Times.“ Der Versuch von Herman, Story Logic, 27-51 in gröBerer Kontinuität zu Vendler narrative Genres auf der Grundlage von Situationstypen zu unterscheiden wird von Hühn, „Event and Eventfulness“ abgelehnt.

12 Siehe dazu detaillierter unten, Abschnitt 1.5.5.

13 Vgl. etwa Breindl, „Grundlagen,“ 121.

14 Es ist zu beachten, dass etwa der Duden unter „Aktionalität“ etwas anderes versteht. Siehe unten, Abschnitt 1.3.

15 Vgl. ausführlich Bache, Study, 217.

16 Bache, Study, 221: ,actional potential.“ 


\subsection{Differenzierung im Duden}

Wenn im Folgenden in Anlehnung an den Duden im Einzelfall von den Aktionsarten von „Verben“ die Rede ist, so ist damit also eigentlich auf die „Zusammenhänge zwischen dem vom Verb [bzw. der Verbkonstellation] bezeichneten Geschehen oder Sachverhalt und dem damit verbundenen internen Zeitverlauf“ Bezug genommen, der sich auf lexikalischer Ebene in (teilweise auch morphologisch) abgrenzbaren Verbklassen widerspiegelt. ${ }^{17}$

Der Duden differenziert grundsätzlich zwischen telischen und atelischen Verben, ${ }^{18}$ wobei die Charakterisierung als „punktuell oder notwendig zeitbegrenzt“ mit der erstgenannten Kategorie verbunden und mit dem Vorhandensein eines expliziten Kulminations- oder Endpunktes verbunden wird. ${ }^{19}$

Als deutlichste Vertreter der telischen Verben werden die „transformativen Verben“ betrachtet, welche „einen Wechsel von einem Zustand (leben, wach sein, an einem bestimmten Ort X wohnen) oder das Verursachen eines solchen Wandels bezeichnen.“20 Die entsprechenden Verben können intransitiv („sterben“), reflexiv („sich öffnen“) oder transitiv (,,jemanden töten“) sein.

Innerhalb der atelischen Verben differenziert der Duden zwischen statischen und dynamischen Verben, ${ }^{21}$ also zwischen „Zuständen“ und „Relationen“ auf der einen Seite und „Vorgängen,“ „Prozessen“ und „Aktivitäten“ auf der anderen Seite. Als spezielle Klasse werden im Duden außerdem die „kontinuativen Verben“ genannt, welche „explizit das Nichteintreten einer Zustandsänderung beschreiben,“ wie etwa „bleiben“ oder „behalten.“22

17 Heinold, Tempus, 25 sieht hier einen Hauptunterschied zwischen der Duden-Tradition und der englischsprachigen Literatur zu Zeitschemata im Gefolge Vendlers. Siehe aber auch Duden 567. Bereits in $\S 565$ werden Akkusativobjekte mit angegeben.

18 Duden 565.

19 Vgl. Duden 566: „Dass atelische Verben keinen Kulminationspunkt verlangen, bedeutet nicht, dass die entsprechenden Sachverhalte in Wirklichkeit von unbegrenzter Dauer sind. Man kann aber feststellen, dass jemand z. B. zu einer bestimmten Zeit schläft/schlief, ohne den Anfang oder das Endes des Schlafes mit einbeziehen zu müssen. Bei den telischen Entsprechungen einschlafen und aufwachsen hingegen gehört der Umschlagpunkt, der Übergang von einem in den anderen Zustand, oder die Vorstellung davon mit dazu, damit man sagen kann, dass jemand zu einer bestimmten Zeit einschläft/einschlief oder aufwacht(e).“

20 Duden 565. Bei Bezug auf das Eintreten des neuen Zustands werden sie „ingressiv“ bzw. „,inchoativ“ genannt, bei Bezug auf das Aufhören des vorhergehenden Zustandes „egressiv.“

21 Bei Aubrey ist „dynamicity“ ein zusätzliches Kriterium zur Durativität, welche er durch das Gegenstück ,punctual“ aufgreift.

22 Duden 566. 
Zwischen Vorgängen (auf die wohl auch mit dem Terminus „Prozess“ Bezug genommen wird) auf der einen Seite und Aktivitäten auf der anderen Seite kann dann aber nur unterschieden werden, indem weitere Aspekte berücksichtigt werden, die „vor allem an der semantischen Rolle des Subjektaktanten“ orientiert sind. ${ }^{23}$ Der Duden verwendet hierfür das Etikett der „Aktionalität,“ weil er diesen Parameter als über die Aktionsart hinausgehend ansieht.

Auf diese Weise können „Vorgangsverben“ abgegrenzt werden: „sie beschreiben Sachverhalte, die nicht statisch sind und die auch nicht unter der Kontrolle eines Agens stehen.“ Im letztgenannten Punkt unterscheiden sie sich von Aktivitätsverben, welche „dem Subjekt eine typische Agensrolle zu[weisen].“24 Die Aktivitätsverben haben als telisches Gegenstück die „Handlungsverben.“ Es ergibt sich aufgrund des bisher Angesprochenen folgende Übersicht (Abb. 28):

\begin{tabular}{|l|l|l|l|}
\hline \multirow{2}{*}{ Verbklasse } & \multicolumn{2}{|l|}{ Aktionsart } & Aktionalität \\
\cline { 2 - 4 } & Telisch & Statisch & Agentiv \\
\hline Handlungsverben & + & - & + \\
\hline Vorgangsverben & $+/-$ & - & - \\
\hline Tätigkeitsverben & - & - & + \\
\hline Zustandsverben & - & + & - \\
\hline
\end{tabular}

Abb. 28: Übersicht über die Verbklassen im Duden.

Zusätzlich werden unter der Überschrift der Aktionalität auch noch die kausativen Verben diskutiert, bei welchen „der Subjektaktant einen Vorgang - im typischen Fall eine Zustandsveränderung - verursacht, der den Objektaktanten als Patiens involviert. “25 Kausative Verben sind häufig (agentive) Handlungsverben. ${ }^{26}$ Dem Subjekt kann bei manchen Verben aber auch schlicht die Rolle des „nicht (willentlich) handelnden Auslösers der Zustandsänderung zufallen,“ wie dies etwa bei folgendem kausativem Vorgang der Fall ist: „Die Sonne trocknete die Kleider.“ “27

23 Duden 570.

24 Duden 570.

25 Duden 572.

26 Duden 572.

27 Duden 572. 


\subsection{Differenzierung in „The Greek Verb Revisited“}

\subsubsection{Die Zeit-Schemata nach Vendler}

Grundlegend für die Differenzierung zwischen verschiedenen Aktionsarten in der angelsächsischen neutestamentlichen Wissenschaft ist die etwa von den meisten Autoren in The Greek Verb Revisited übernommene ${ }^{28}$ Differenzierung von Vendler zwischen verschiedenen von Verben vorausgesetzten „time schemata,“29 welche sich anhand von drei Parametern differenzieren lassen.

Die folgende Abb. $29^{30}$ stellt diese dar, wobei außerdem noch die Erweiterung um „semelfactives,“ die Mike Aubrey im selben Band aufnimmt, integriert wurde: ${ }^{31}$

\begin{tabular}{|l|l|l|l||l|}
\hline Aktionsart & \multicolumn{3}{|c||}{ Kriterien } & Beispiele \\
\hline & Stativität & Durativität & Telizität & \\
\hline State & + & + & - & {$[$ [Christoph lieben Theresa $]$} \\
\hline Activity & - & + & - & {$[$ [Christoph rennen] } \\
\hline Accomplishment & - & + & + & {$[$ [Christoph rennen einen Kilometer] } \\
\hline $\begin{array}{l}\text { Achievement (mit } \\
\text { und ohne ,preface“) }\end{array}$ & - & - & + & $\begin{array}{l}\text { [Christoph gewinnen Marathon }] \\
\text { [Christoph zerbrechen Glas] }\end{array}$ \\
\hline Semelfactives & - & - & - & {$[$ Christoph werfen Ball] } \\
\hline
\end{tabular}

Abb. 29: Übersicht über Aktionsarten in Anlehnung an Vendler.

28 In Fresch und Runge, Hg., Greek Verb etwa Thomson, „Aspect,“ 49, Crellin, „Semantics, “ 436, Moser, „Tense and Aspect,“ 449. Siehe auch bereits Fanning, Verbal Aspect, 128-163. Dort finden sich auch zahlreiche (teilweise diskussionswürdige) Beispiele.

29 Vendler, „Verbs and Times,“ 143.

30 Die Tabelle orientiert sich an Thomson, „Aspect,“ 49. Teilweise werden Vendlers Kategorien auch an anderen Kriterien festgemacht. So werden recht häufig etwa Zustände und Aktivitäten als gemeinsam „homogen“ (d. h. beliebig fortsetzbar) gesehen, aber dadurch unterschieden, dass nur Aktivitäten (zusammen mit den „accomplishments“) auch „phasisch“ (d.h. in Phasen zerlegbar) seien. Terminologisch und was die Darbietung der Beispiele angeht, wurde die Tabelle hier jedoch der besseren Verständlichkeit wegen direkt an die hier vorausgesetzte Terminologie angepasst. Crellin, „Semantics,“ 436 unterscheidet außerdem noch zwischen schwacher und starker Homogenität, wobei letztere meint, dass „an eventuality is infintely subdividable into events of the same character.“ Diese Eigenschaft kommt dann nur Zuständen zu.

31 Aubrey, „Prohibitions,“ 498-500. 
„States“ und „activities“ - Zustände und Aktivitäten (bzw. im Deutschen wohl besser „Prozesse“ im Fall eines Agens ohne Bewusstsein) - haben keinen natürlichen Endpunkt und sind nicht-punktuell, ${ }^{32}$ unterscheiden sich aber darin, dass nur Aktivitäten dynamisch sind.

„Accomplishments“ und „Achievements“ hingegen weisen beide einen Kulminationspunkt auf - vor dessen Erreichen kann also eine entsprechende Proposition nicht wahrheitsgetreu ausgesagt werden. Sie unterscheiden sich darin, dass „achievements“ (mehr oder weniger) punktuell sind: Der Martathon wird im Moment des Überschreitens der Ziellinie gewonnen, das Rennen der Strecke von einem Kilometer wird hingegen längere Zeit in Anspruch nehmen. ${ }^{33}$ Eine durchgängig etablierte deutsche Terminologie für diese beiden Kategorien Vendlers gibt es leider nicht. ${ }^{34}$

\subsection{2 „Semelfactives“}

Die Einführung der „semelfactives“ als weitere Kategorie geht auf Smith zurück, die sie wie folgt beschreibt: „Semelfactives are single-stage events with no result or outcome ... Semelfactives are the simplest type of event, consisting only in the occurrence." ${ }^{35}$ Die semelfaktive Aktionsart wird von anderen Autoren, die im ursprünglichen Rahmen von Vendler bleiben, den ,achievements“ zugerechnet. So schreibt Thomson: „Some achievements, for example [the door open], involve the subject ending up in a new resulting state. Others, such as [the light flash] do not.“" ${ }^{36}$ Da sie in der linguistischen Literatur jedoch als nicht-telisch gelten, werden sie hier separat behandelt - aber in die die ursprünglichen Kategorien integrierende Tabelle eingefügt, da sie sich anhand der vorgegebenen Merkmalskominatorik abgrenzen lassen.

32 Vgl. die etwas andere Aufteilung bei Crellin, „Semantics,“ 437.

33 Crellin, „Semantics,“ 437 unterscheidet vom Situationstyp ,accomplishment“ die von ihm als „complex events“ bezeichnete Situation, in welcher zwar vom Subjekt ein neuer Zustand erreicht wird, aber zugleich gilt: „This state has no set endpoint.“ Beispiel: [Banane verrotten].

34 Breindl, „Temporalität, Aspektualität und Modalität,“ 222 übersetzt etwa „achievements“ mit „Ereignissen“ und „accomplishments“ mit „Handlungen.“ Letztere Bezeichnung ist problematisch im Fall, dass keine bewusst agierende Entität involviert ist (z. B.: „Das Eis schmolz“). Die erste Glosse ist in unserem Kontext problematisch, da erzähltheoretisch meist von „Ereignissen“ im allgemeineren Sinn von ,Situationen` die Rede ist (vgl. dazu auch oben, Abschnitt 1.1 zur Terminologie von Heinrich von Siebenthal).

35 Smith, Parameter, 29.

36 Thomson, „Aspect,“55-56. Hervorhebung hinzugefügt. 


\subsection{3 „Achievements“ mit und ohne „preface“}

Eine weitere Differenzierung innerhalb der „achievements“ betrifft die Frage, ob das eigentlich punktuelle Ereignis mit einem „preface“ ${ }^{\text {“37 }} \mathrm{zu}$ denken ist: [Christoph finden Mantel] impliziert beispielsweise einen länger anhaltenden Prozess des Suchens, auch wenn das Finden selbst punktuell ist. ${ }^{38}$ Bei [Christoph zerbrechen Glas] ist hingegen in der Regel nicht an eine vorangehende Aktivität gedacht, es liegt lediglich eine sehr punktuelle Transformation des Glases von einem Zustand in einen anderen vor. Im Anschluss an Fanning nennt Thomson diese Unterkategorie „climaxes.“"39

\subsection{4 „Active accomplishments“}

Aubrey fügt außerdem - nicht in der Tabelle enthalten - die Kategorie der ,active accomplishments“ hinzu, ${ }^{40}$ die er von Van Valin übernimmt, der hier den telischen Gebrauch von Aktivitätsverben versteht. ${ }^{41}$ Während [Christoph rennen] eine Aktivität darstellt, ist in [Christoph rennen einen Kilometer] ein Kulminationspunkt enthalten: Vor erreichen dieser Strecke wäre die Proposition nicht wahr.

Die Kategorie ist nicht mit in die Tabelle übernommen worden, da sie sich nicht anhand der bestehenden Kriterien etablieren lässt. Thomson, der im Rahmen der ursprünglichen Kategorisierung von Vendler arbeitet, weist entsprechend darauf hin, dass manche ,accomplishments“ durch Hinzufügen von Endpunkten zu Aktivitäten gebildet werden..$^{42}$ Lagert man mit Van Valin solche Fälle als separate Kategorie aus, so betrifft dies auch Verbkonstellationen wie [Noah bauen Arche], da das Vorgangskonzept auch hier zusammengesetzt ist. ${ }^{43} \mathrm{Nicht}$ betroffen von Van Valins Klassifizierungssystem wäre etwa die nicht-transitive

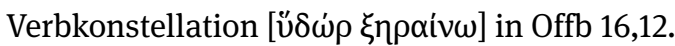

Um die „active accomplishments“ von den „accomplishments“ zu trennen, muss ein weiteres Kriterium hinzugefügt werden, das der „Dynamizität,“ welches nicht so intuitiv zu fassen ist, wie die anderen Kriterien: „The feature ,dynamic’

37 Smith, Parameter, 31 spricht von einem „preliminary stage.“

38 Thomson, „Aspect,“ 58.

39 Fanning, Verbal Aspect, 129.

40 Aubrey, „Prohibitions,“ 499.

41 Van Valin, Syntax, 32: „This general pattern relates activity verbs of motion (e.g. run), consumption (e.g. eat) and creation (e.g. paint) to the corresponding active accomplishment verbs.“

42 Vgl. Thomson, „Aspect,“53-55.

43 Vgl. Vendler, Syntax, 33 mit Beispielen für Verben des Konstruierens und Konsumierens. 
refers to whether the situation involves action or not." ${ }^{44}$ Auf diese Eigenschaft hin kann getestet werden, indem man das Verb mit Adverbien wie „violently“ versuchsweise ergänzt. Damit soll aber nicht Agentivität eingeführt werden, wie Van Valin explizit festhält. ${ }^{45}$ Es muss beachtet werden, dass in der Literatur Dynamizität häufig schlicht das Gegenstück zur Stativität darstellt. ${ }^{46}$

\subsubsection{Kausative Varianten}

Außerdem merkt Aubrey im Anschluss an Van Valin an, dass zu jeder Kategorie kausative Varianten gebildet werden können (Abb. 30):47

\begin{tabular}{|l|l|l|}
\hline Aktionsart & Beispielsatz & Kausative Variante \\
\hline State & Tucker was terrified. & Pierre terrifies Tucker. \\
\hline Activity & $\begin{array}{l}\text { Dave walked around the } \\
\text { park. }\end{array}$ & $\begin{array}{l}\text { Dave walked his dog in the } \\
\text { park. }\end{array}$ \\
\hline Accomplishment & The door opened abruptly. & $\begin{array}{l}\text { Rachel opened the door } \\
\text { slowly. }\end{array}$ \\
\hline Active achievement & $\begin{array}{l}\text { The soldiers marched to } \\
\text { the park. }\end{array}$ & $\begin{array}{l}\text { The captain marched the } \\
\text { soldiers to the park. }\end{array}$ \\
\hline Achievement & $\begin{array}{l}\text { The car crashed into the } \\
\text { barrier. }\end{array}$ & $\begin{array}{l}\text { Dave crashed the car into } \\
\text { the barrier. }\end{array}$ \\
\hline Semelfactives & $\begin{array}{l}\text { The lightening flashed in } \\
\text { the night. }\end{array}$ & $\begin{array}{l}\text { Henry flashed his } \\
\text { headlights at another car. }\end{array}$ \\
\hline
\end{tabular}

Abb. 30: Kausative Varianten der klassischen Aktionsarten.

Die kausativen Verben weisen eine komplexe Struktur auf: Es werden hier zwei Prädikate kombiniert - eines (in der Regel eine Aktivität), welches das verursachende Geschehen anzeigt und eines, welches die resultierende Situation identifiziert. ${ }^{48}$ Die ursprünglichen Vendler'schen Kategorien sind für solche zusammengesetzten Prädikatstypen nur wenig geeignet (vgl. die im Duden daher angeführten transitiven Transformationsverben bzw. Kausativität als Faktor der Aktionalität). Entsprechend umstritten ist die Integration kausativer Varianten

44 Van Valin, Syntax, 33.

45 Van Valin, Syntax, 36. Die verwendeten Adverbien „that code dynamic action“ müssen daher mit „involunaty verbs“ verbindbar sein, (anders als „carefully“ etc.).

46 Vgl. Duden 566.

47 Die Tabelle basiert auf den Beispielen von Aubrey, „Prohibitions,“ 50, der sich wiederum an Van Valin, Syntax, 34. Für den entsprechenden Test für die Feststellung der Kausativität siehe Van Valin, Syntax, 38-39.

48 Van Valin, Syntax, 42. 
in der Literatur, die mit dem ursprünglichen Set an Situationstypen arbeitet. Annäherungsweise lassen sie sich in der Regel jedoch der Kategorie zuordnen, von welcher sie abgeleitet sind (d.h., ein ,active achievement“ kann als spezielle Ausprägung eines ,achievement“ verstanden werden etc.). ${ }^{49}$

Gerade für den Resultativ ist die Kategorie der Transitivität von zentraler Bedeutung. Zugleich soll die Darstellung hier jedoch nicht unnötig verkompliziert werden. Was Durativ und Aorist angeht, lässt sich das Zusammenspiel von grammatischem Aspekt und dem Situationstyps-Potenzial der Verben befriedigend anhand der ursprünglichen Kategorien Vendlers explizieren. Auf den Zusammenhang zwischen der im Resultativ dargestellten Situation und der (u. U. kausativen) Rolle eines Agens in dieser Situation soll daher gesondert eingegangen werden (siehe unten, Abschnitt 2.2.3), die kausativen Varianten werden ansonsten jedoch nicht als Kategorien vorausgesetzt.

\subsection{Auswertung}

\subsubsection{Synthese}

Für die narratologische Analyse von verschiedenen im Text zur Sprache kommenden Situationen ist die am Duden orientierte Differenzierung zwischen Aktivitäten, Zuständen, Vorgängen und Handlungen hilfreich. Die Zuordnung von Aktivitäten zu Vendlers ,activities“ und Zuständen zu „states“ ist dabei unproblematisch.

Handlungen und Vorgänge unterscheiden sich aber anhand der Gegenüberstellung agentiv/nicht-agentiv und umfassen bei Vendler ,accomplishments“ und „achievements,“ welche dort anders, nämlich über das Gegensatzpaar „durativ/punktuell,“ differenziert werden. Auch die gegenüber „achievements“ nicht-telischen (aber ebenso punktuellen) und gegenüber „accomplishments“ nicht-durativen (aber ebenso atelischen) „semelfactives“ sind entsprechend hier einzuordnen. Da kausative Verben meist (agentive) Handlungen darstellen, können zumindest die kausativen Varianten der Vendler'schen Klassen tendenziell mit der Duden-Kategorie der Handlungsverben assoziiert werden.

Für die Merkmalskombination + telisch, - statisch, - durativ, - agentiv, - wohl Heinrich von Siebenthals „Ereignis“ (z. B. [Tasse fallen zu Boden]) - fehlt leider in beiden Systemen eine Kategorie. Bei diesem Erzählungen durchaus häufigen Situationstyp handelt es sich also um - wie man umständlich sagen müsste „nicht-agentive achievements“ bzw. um „telische (und punktuelle) Vorgänge.“ Es zeigt sich hier zweifellos, was der Duden in einer Fußnote zur Thematik einräumt:

49 Vgl. die Distribution der Testergebenisse bei Van Valin, Syntax, 39. 
„Generell ist die Fachterminologie zum Thema Aktionsart sehr uneinheitlich und verwirrend. “50

\subsubsection{Handlungsverben und Erzählungen}

Bei allen Schwierigkeiten, die bei der Differenzierung vom erzählten Situationen bestehen bleiben, ergibt sich aus narratologischer Sicht doch ein deutlich erkennbarer Nutzen der hier angerissenen Erwägungen, da sich vor diesem Hintergrund die oben problematisierte Ereignis-Bedingung (Kapitel 3, Abschnitt 3.4.3.3) nun konkreter erfassen lässt.

Unter Bezugnahme auf die Aktionsart der Verben lässt sich nun für eine prototypische Erzählung nach Ryan spezifischer die Bedingung ableiten, dass ein Text auf seiner grammatisch-lexikalischen Ebene (mit den Duden-Kategorien gesprochen) auch Handlungsverben oder Tätigkeitsverben aufweisen muss. Für die minimalistische Definition von Köppe und Kindt gilt dies - wie schon das Beispiel des fallenden Apfels zeigt - nicht.

Erst in der Stilisierung des Geschehens zum „kleine[n] persönliche[n] Drama“ des Apfels („Erst hielt sich der Apfel noch am Baum, dann stürzte er, von seinen Kräften verlassen, ab.“)51 kommen Handlungsverben ins Spiel. Aufschlussreich ist, dass Köppe und Kindt diese Umformulierung im Rahmen der Frage nach den Kriterien für Erzählungen im gehaltvolleren Sinn vornehmen, um nämlich die Bedingung der „Ereignisreferenz“ zu illustrieren. Handlungsverben stellen auf jeden Fall ein wichtiges Mittel zu deren Realisierung dar. Absolut notwendig scheinen sie jedoch nicht. Ein ähnlicher Effekt kann durch Verweis auf mentale Zustände erzielt werden („Er sorgte sich um den Apfel ...“). Wenn Köppe und Kindt schreiben, dass die Umformulierung klarmache, „dass es sich, aus der Perspektive des Protagonisten [d.h.: des Apfels], um ein kleines persönliches Drama handelt, "52 so gilt das natürlich auch für ein Erzählen der Situation mit interner Fokalisierung auf Erzählfiguren, die dem Geschehen beiwohnen.

\subsubsection{Zustandsverben und Erzählungen}

Etwas schwieriger ist die Beurteilung der Zustandsverben im Hinblick auf die Ereignis-Bedingung. Das Beispiel der Minimalerzählung über einen Apfel zeigt, dass

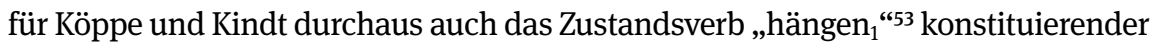

50 Duden 566.

51 Köppe und Kindt, Erzähltheorie, 64-65.

52 Köppe und Kindt, Erzähltheorie, 65.

53 Das Verb hat die Bedeutung $(e W D G)$, an einer bestimmten Stelle oben befestigt und nach unten schwebend sein.‘ Es ist natürlich nicht zu verwechseln mit „hängen ${ }_{2}$,“ welches ,etw., 
Teil einer Erzählung sein kann. Auch ein Text, der ausschließlich aus Zustandsverben besteht, könnte sinnvoll als Erzählung aufgefasst werden, wenn der Wechsel zwischen den Zuständen nachvollziehbar bleibt. Eine derartige Umformulierung der Apfel-Geschichte scheint in der Tat möglich: „Erst hing der Apfel am Baum. Dann lag er auf dem Boden." 54

\subsubsection{Ein Verb, mehrere Ereignisse?}

An dieser Stelle kann die Forderung nach mindestens „zwei Ereignissen,“ von denen ein Text $\mathrm{zu}$ handeln habe, um als Erzählung gelten zu können, unter Rückgriff auf die Verbsemantik spezifiziert werden. Auf der Ebene der Erzählung (des „discourse“) kann in der Tat ein einzelnes Verb genügen, wenn dieses einen „Erzählschritt“ zum Ausdruck bringt - welchen der Duden als „Transformation eines Ausgangszustands zu einem Endzustand ..., wobei die beiden Zustände eine inhaltlich bedeutsame Differenz unterscheidet“ definiert. ${ }^{55}$

Dies ist zweifellos bei transformativen Verben der Fall, da hier der ins Gegenteil führende Zustandswechsel definitionsgemäß signifikant ist (z. B.: „Er schnitt den Apfel ab.“). Transformative Verben können sowohl transitiv als auch intransitiv sein, also auch sowohl agentiv wie auch nicht-agentiv - und damit zu den Kategorien der Handlungen und (telischen) Vorgänge gehören. ${ }^{56}$ Auch der Satz „Der Apfel fiel vom Baum herunter.“ impliziert somit das ins Gegenteil verkehrte vorherige Hängen und kann als Erzählung gewertet werden.

In der Vendler'schen Kategorisierung kommen einzelne Sätze, die ,accomplishments“ und „achievements“ (ohne „semelfactives“) zum Ausdruck bringen, in Frage, da ihre Telizität einen Zustand der Abgeschlossenheit impliziert. Bei Achievements kann die Existenz eines impliziten „preface“ (z. B. Suchen vor dem Moment des Findens) die Wahrnehmung eines entsprechenden Einzelsatzes als Erzählung erleichtern. ${ }^{57}$ Dieser kann jedoch auch wieder dem Ausgangszustand entsprechen. ${ }^{58}$

jmdn. an einer bestimmten Stelle oben befestigen und nach unten schweben lassen ' bedeutet und damit zu den kausativen Verben gehört (Duden 572).

54 Hier wird natürlich die Möglichkeit offen gelassen, dass das in der erzählten Welt die beiden Zustände verbindende Geschehen nicht in einem Fallen besteht, sondern z. B. in einem Pflücken und Ablegen.

55 Duden 1925.

56 Vgl. Duden 565.

57 Wobei ohne Kontext die implizierte vorausgehende Aktivität nicht unbedingt gegeben ist: „Christoph fand einen Apfel.“ Der Finder kann auch zufällig über ihn gestolpert sein. Vgl. Thomson, „Aspect,“ 58.

58 Vgl. Thomson, „Aspect,“ 54, welcher das Rennen einer Meile mit voraus- und nachfolgendem „rest state“ einträgt. Identisch sind die Zustände allerdings nicht, da das Agens nach Ende 
Die Entscheidung ist letztlich vom Ausmaß der erzielten Veränderung abhängig,59 wobei das erzielte Resultat (vgl. Abb. 31) ganz unterschiedlicher Art sein kann: ${ }^{60}$

\begin{tabular}{|l|l|l|}
\hline Resultatstyp & Accomplishments & Achievements \\
\hline Affected object & $\begin{array}{l}\text { [bend an iron bar], [wrinkle } \\
\text { a dress], [break a pot] }\end{array}$ & [break a cup], [tear a paper] \\
\hline Constructed object & $\begin{array}{l}\text { [build a house], [write a } \\
\text { letter] }\end{array}$ & $\begin{array}{l}\text { [imagine a city], [define a } \\
\text { parameter] }\end{array}$ \\
\hline Consumed object & $\begin{array}{l}\text { [destroy a house], [drink a } \\
\text { glass of wine] }\end{array}$ & [explode a bomb] \\
\hline Affected experiencer & [amuse Mary] & [see a comet] \\
\hline Path-Goal & $\begin{array}{l}\text { [walk to lake], [work from } \\
\text { 2 to 3] }\end{array}$ & $\begin{array}{l}\text { [reach the top], [arrive in } \\
\text { Boston] }\end{array}$ \\
\hline
\end{tabular}

Abb. 31: Verschiedene Typen an erzielten Resultaten.

\subsubsection{Tatsächliche Situationen und Situationstypen}

Über die definitorische Frage hinaus ist für die narratologische Aufgabe die Diskussion zur Aktionsart auch noch von anderer ganz grundsätzlicher Bedeutung: Die Einteilungen in verschiedene Typen an Situationen „do not classify states of affairs objectively existing in the real world, or conceptions of states of affairs, but sets of of temporal characteristics communicated by the verb constellation. "61

Es besteht einerseits durchaus ein Zusammenhang zwischen der Wahrnehmung tatsächlich erfahrener Situationen und den im sprachlichen Bereich möglichen Kategorisierungen. ${ }^{62}$ Unter anderem aufgrund dieser Verankerung in der

der Situation zumindest die Eigenschaft aufweist, besagte Situation als Teil seines „Erfahrungsschatzes“ vorweisen zu können. Ein „Olympionike“ ist man erst nach der Teilnahme an einer Olympiade, auch wenn man danach wieder zum selben Trainingsprogramm zurückkehrt. Vgl. unten, Abschnitt 2.2.3 beim Resultativ zu dieser Eigenschaft des Agens.

59 In der Darstellung von Thomson, „Aspect,“55-59 ließe sich dies durch Variation der Entfernung der beiden Balken markieren.

60 Vgl. Smith, Parameter, $27 \mathrm{zu}$,accomplishments.“

61 Thomson, „Aspect,“ 49.

62 Smith, Parameters, 6-7: „The situation types of a language represent idealized situations which are grammaticized in the language ... The situation types are idealizations, drawn from the temporal classifications of actual situations that people make on perceptual and cognitive grounds.“ Vgl. S. 27: „I will use the names Stative, Activity etc. to refer to idealized situation types, to sentences associated with them, and to situations in the world. Thus in talking about an actual situation that I perceive as Stative, I may utter a Stative sentence; the linguistic forms are associated with the stereotype and temporal schema of the Stative situation type. The situation types are realized by constellations of lexical morphemes consisting of a verb and its arguments, including the subject (I assume that the subjects are verb arguments.“ Vgl. auch bereits Bache, 
Erfahrung gilt auch der übereinzelsprachliche Anspruch ${ }^{63}$ der Klassifizierungen - und ergibt sich die oben kurz angerissene Modifikation der ursprünglichen Einteilung nach Vendler.

Auf der anderen Seite ist das Verhältnis von tatsächlicher Situation und Situationstyp über den Sprecher vermittelt. Der Sprecher präsentiert ${ }^{64}$ eine tatsächliche Situation als Exemplar eines bestimmten Situationstyps durch die Wahl einer mit dem Situationstyp korrelierenden Verbkonstellation. ${ }^{65}$ Dabei greift der Sprecher auf Kategorien zurück (Telizität, Durativität etc.), welche nicht objektiv in der realen Situation vorliegen, sondern dieser innerhalb eines bestimmten psychologischen Bewertungssystemes zugeschrieben werden. ${ }^{66}$ Auch im Fall universeller Übereinstimmungen ${ }^{67}$ über die Beschreibung und Klassifizierung von tatsächlichen Situationen (z. B. als punktuell) besteht der Bezug letztlich auf eine konzeptuelle Ebene, nicht direkt auf die Realität. ${ }^{68}$

Die mit der Aktionsart (bzw. dem ,lexical aspect“ im Gegenüber zum „grammatical aspect“) teilweise verbundene Bezeichnung als „objektiv“ im Gegensatz zum „subjektiven“ Aspekt ist daher zumindest missverständlich. So etwa in der CGCG: „As opposed to grammatical aspect, lexical aspect thus has to do with the objective properties of an action, rather than with subjective ways of presenting that action." 69 Beispielsweise kann auf dieselbe tatsächliche (z. B. beobachtete) Situation mit den folgenden beiden Sätzen Bezug genommen werden: „The ship moved.“ und „The ship was in motion. “70 Die Sätze realisieren zwei grundsätzlich

„Aspect and Aktionsart,“ 61: „... in our conception of the world ... “ Spezifischer S. 70: „These notions [d. h.: Gegensatzpaare wie telisch/atelisch etc.] are not to be regarded as physically measurable, ,objective characteristics of situations but rather as psychological classifications of (objective and other) situations based on intuitive belief or conception.“

63 Vgl. Smith, Paramter, 2. Vgl. auch S. XV.

64 Vgl. grundsätzlich Bache, „Aspect and Aktionsart,“ 64-71 für diese Dichotomie, die zumindest um ein „quasi-“ ergänzt werden muss (S. 71). Zum „objektiven“ Charakter der Aktionsart: "[It] is rather of a psychological nature, involving the speaker/writer's conception of situations and corresponding semantic properties of verbs.“

65 Smith, Parameter, 7: „The speaker links an actual situation to a meaning by talking about the situation with a particular set of linguistic forms, a verb constellation. The relation between situations and sentences is indirect: it is mediated by the speaker. The speaker, not the situation, determines aspectual choice. “ Smith meint mit ,aspectual choice“ the Kombination aus Situationstyp (Aktionsart) und Viewpoint (Aspekt).

66 Siehe Bache, Study, 53-54.

67 Im Rahmen hochspezifischer Betrachtungsweisen (siehe unten zur Physik) ist grundsätzlich immer mit abweichenden Konzeptualisierungen zu rechnen.

68 Bache, Study, 55-56.

69 Vgl. CGCG 33.8 (ursprünglich fette Hervorhebung kursiv gesetzt).

70 Smith, Parameter, 6. 
verschiedene Situationstypen (Aktivität vs. Zustand), ohne dass jedoch einer der beiden unangemessen oder irreführend ${ }^{71}$ wäre.

Zugleich kann aber durchaus zwischen unkonventioneller und konventioneller Wahl unterschieden werden: „The former is the conventional choice, according to the properties that are salient perceptually and functionally. The latter has a special effect: it freezes the motion."72 Die Wortwahl des Erzählers kann also für dieselbe erzählte Situation verschiedene Situationstypen evozieren, wobei unkonventionelle Entscheidungen auf spezifische Erzählinteressen hindeuten, beispielsweise auf die Konzeptualisierung eines Geschehens als eines anhaltenden Hintergrundes für ein im Vordergrund stehendes Ereignis. ${ }^{73}$

Die kulturelle Konventionalisierung betrifft auch die Detail-Ebene, auf welcher der Zugriff auf ein Ereignis gewöhnlich erfolgt: Die tatsächliche Situation eines Niesens wird normalerweise als ein einzelnes Ereignis verstanden und entsprechend durch die Verbkonstellation [S niesen] dargestellt. Eine detailliertere Auflösung der Situation ist gegenüber dieser „basic-level“ Kategorisierung aber grundsätzlich möglich und dann „markiert.“74 Die Konventionen sind natürlich auch kontext- und damit genreabhängig. Selbst ein normalerweise als „punktuell“ konzeptualisiertes Ereignis wie ein Blitz (ein „semelfactive“) kann z. B. in einem Physik-Lehrbuch in verschiedene Phasen zerteilt vorliegen.

\section{Grammatischer Aspekt}

\subsection{Hinführung}

Die Rede vom grammatischen Aspekt erfolgt oft unter Bezugnahme auf visuelle Metaphern, wie etwa „Viewpoint“ oder „Perspektive.“75 Das hat in der neutestamentlichen Exegese teilweise zu dem Missverständnis geführt, die Kategorie des Aspekts habe keine zeitliche Komponente. ${ }^{76}$ Thomson kommentiert diesen Sprachgebrauch daher wie folgt:

71 Smith, Parameter, 7: „Note that the receivers will not be misled by unconventional choice.“ 72 Smith, Parameter, 7.

73 Siehe auch unten, Abschnitt 3.3.4 für die Rolle des grammatischen Aspekts für diese „Reliefgebung.“

74 Smith, Parameter, 10-11 mit dem Beispiel „Daniel is walking.“ vs. „Daniel is taking steps.“

75 Vgl. Duden 569 zu „Außen- und Binnenperspektive.“ Die Autoren verzichten dafür aber auf die Rede vom „Aspekt.“

76 Siehe Thomson, „Aspect,“ 38-48 für eine entsprechende Kritik. 
If one must use the language of ,view“ then aspect is in fact more to do with what is in view whether the entire course of a situation (perfective aspect) or a phase excluding endpoints (imperfective aspect) - than the position or viewpoint one views it from. But the language of ,viewing“ is itself problematic, because a speaker or writer does not use language to view situations, but rather to speak about them. To be sure, language sometimes creates a mental picture. But that is a pragmatic effect arising from the way it is used in context, and aspectual forms can be used in abstract prose that does not create any mental picture whatsoever.

Es wird unten (Abschnitt 3) im Hinblick auf das Konzept des ,Tempus‘ noch auf das Verhältnis von Sprechzeit und Ereigniszeit ${ }^{77}$ eingegangen werden. Die Kategorie des Aspekts bezieht sich demgegenüber auf das Verhältnis von Ereigniszeit auf der einen Seite und Betrachtzeit (,,reference time“; in den Abbildungen: RT; andere verwenden die Bezeichnung ,topic time“"78) auf der anderen. ${ }^{79}$ Auch der grammatische Aspekt hat also etwas mit Zeit zu tun, aber eben mit der „situationsinternen.“80

Auf der Inhaltsseite des Textes entspricht der grammatischen Kategorie des Aspekts das inhaltliche Gegenstück der „Aspektualität.“ Auch das Deutsche, welches keine Aspekt-Sprache ist, kann Aspektualität sehr wohl zum Ausdruck bringen, muss dies jedoch durch (a) Umschreibung oder (b) durch lexikalische Stützung tun. ${ }^{81} \mathrm{Im}$ Englischen wird durch die Unterscheidung von „simple past“ und „continuous past“ wie im Griechischen eine Aspekt-Unterscheidung vorgenommen. ${ }^{82}$

Im Folgenden sollen die drei im Griechischen vorhandenen Aspektkategorien kurz vorgestellt werden. ${ }^{83}$ Dabei soll auch expliziert werden, wie die Interaktion von Aspekt und Situationstyp ${ }^{84}$ zu spezifischen „Aspektnuancierungen“"85 führt. ${ }^{86}$

77 Es ist dies hier immer im allgemeinen Sinn von Situationszeit gebraucht.

78 Crellin, „Semantics,“ 438.

79 Thomson, „Aspect,“ 35 mit Verweis auf Johnson, „Temporal Theory.“

80 Comrie, Aspect, 5.

81 GGNT 192e mit Beispielen. Vgl. Duden 569. Im „sprechzeitbezogenen Präsens atelischer Verben“ liegt beispielsweise standardmäßig eine Binnenperspektive vor, wobei der „Vorgang ... von einem Punkt aus gesehen [wird], der zeitlich in ihr enthalten ist.“ Telische Verben hingegen erlauben es nicht ohne Weiteres, den Kulminationspunkt auszuklammern, sodass hier im Deutschen „spezifische Mittel der Verlaufsform nötig“ sind, wie etwa: „Als Friederike nach Hause kam, war Hans am Aufstehen.“ Zur Formulierung „am Lesen“ in der Widmung vgl. einerseits Duden 569 und 594 zur nicht abgeschlossenen Grammatikalisierung. Siehe andererseits aber auch Gárgyán, „Progressiv im Deutschen.“

82 Siehe GGNT 192c für Beispiele und Unterschiede, was die Aspektualität der Sprachen angeht. 83 Zur gegenüber Modus und Tempus überwiegenden Bedeutung des grammatischen Aspekts im Griechischen siehe Ellis, „Aspect-Prominence.“

84 Bzw. „grammatical aspect“ und „lexical aspect.“

85 GGNT $194 \mathrm{~m}$.

86 Diese werden in der Exegese teilweise als vom Exegeten recht frei wählbare „Aspektbedeutungen“ aufgefasst, was sicherlich auch mit dem nach wie vor unbefriedigenden Stand der 
Dieses Zusammenspiel ist gerade auch für die narratologische Analyse von höchster Bedeutung, da sich die vom Erzähler realisierte Darstellung der Ereignisse primär aus diesen beiden Faktoren ergibt.

\subsection{Die Aspekte im Überblick}

\subsubsection{Durativ}

Der Durativ stellt die Situation aus einer Binnenperspektive heraus dar, also ohne zeitliche Begrenzung. ${ }^{87}$ Mit anderen Worten: Teile der Ereigniszeit reichen über die Betrachtungszeit hinaus. ${ }^{88}$ Der sprachübergreifend als „imperfektive“ bezeichnete Aspekt hat somit einen eventuellen Kulminationspunkt nicht im Blick (bzw. nicht-metaphorisch: bezieht sich nicht auf diesen). ${ }^{89}$ Diese Bezeichnung wird hier jedoch aufgrund des Tempus des Imperfekts (siehe unten, Abschnitt 3.3) eher gemieden. Auch wird aus analogen Gründen der entsprechende Stamm bevorzugt nicht als „Präsens-Stamm“ bezeichnet und stattdessen die Rede vom Durativ bzw. durativen Aspekt/Stamm gewählt..$^{90}$ Dass in der etablierten Kombination mit Modus-Angaben (z. B. „Konjunktiv Präsens“) der Stamm und nicht das Tempus gemeint ist, versteht sich von selbst.

Auch wenn eine telische Verbkonstellation gewählt wird, ist durch die im Durativ implizierte limitierte Betrachtzeit folglich eine Konzentration auf den Verlauf/das Andauern möglich, es liegt also eine „lineare“ Aspektnuancierung vor. ${ }^{91}$ In Kombination mit einem imperfektiven Aspekt kann die Verbkonstellation [S rennen einen Kilometer] auch dann als Satz wahrheitsgetreu ausgesagt werden, wenn die spezifizierte Strecke nicht vollendet wurde. Graphisch lässt

Forschung zur Klassifikation von Verbkonstellationen im Hinblick auf Situationstypen zurückgeführt werden kann. Vgl. weiterhin grundlegend Fanning, Verbal Aspect, 128-163.

87 GGNT 192c.

88 Thomson, „Aspect,“ 35-56.

$89 \mathrm{Vgl}$. CGCG 33.6: „The present stem presents an action as incomplete, focusing on one or more of its intermediate stages, but leaving its boundaries (beginning and end) out of focus.“

90 GGNT 194a. Thomson, „Aspect,“ 39 meint, „durativity“ sei zwar eine notwendige Implikation des imperfektiven Aspektes, aber nicht das charakterisierende Merkmal, welches vielmehr „the lack of reference to endpoints“ sei. Genau das ist aber wohl bei GGNT 194a gemeint (,etwas Nichtabgeschlossenes, sich noch Entwickelndes, Fortdauerndes“). Vgl. auch Thomson, „Aspect,“ 66-67: Der imperfektive Aspekt benötige eine nicht-punktuelle Situation und impliziere daher „duration.“ Jedoch: „Durativity is not generally considered to be the defining characteristic of the imperfective, since it cannot be used to speak of a durative situation including its endpoints.“ 91 GGNT 194b. Vgl. das Beispiel in 194n. 
sich dieser Zusammenhang von Situationstyp (Handlung) ${ }^{92}$ und Aspekt wie folgt in Abb. 32 darstellen: ${ }^{93}$

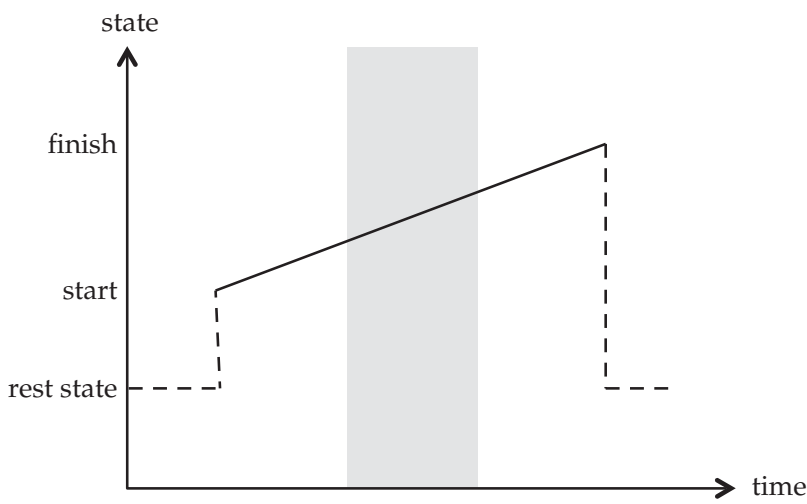

Abb. 32: Verbkonstellation mit durativem und telischem Aktionsartpotential im Durativ.

Die Schräge der Linie zeigt an, dass ein Prozess vorliegt, der auf ein Ziel zuläuft. ${ }^{94}$ Grau unterlegt ist die Betrachtzeit, auf welche sich der Sprecher bezieht. Entspre-

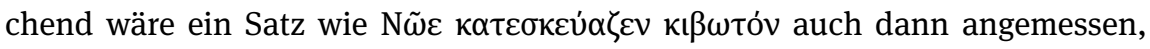
wenn Noah die Arche nicht fertig konstruiert hätte. ${ }^{95}$

Eine konative Aspektnuancierung ergibt sich, wenn der anvisierte ${ }^{96}$ Endpunkt „trotz geeigneter Anstrenungen nicht erreicht“ wird. ${ }^{97}$ Entsprechend kommentiert Thomson Gal 1,13:98

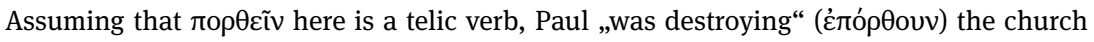

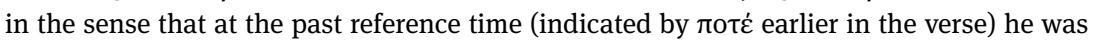
pursuing a project which, if carried on to completion as he intended, would culminate in the extinction of the church.

92 Es handelt sich dabei um ein „accomplishment“ bzw. um ein „,active accomplishment“ (eine Aktivität mit Zielpunkt).

93 Thomson, „Aspect,“ 61.

94 Thomson, „Aspect,“ 54.

95 Thomson, „Aspect,“ 61.

96 Vgl. Thomson, „Aspect,“ 62 zum Problem telischer Vorgänge, d. h. ohne bewussten Agenten.

97 GGNT 194 n.

98 Thomson, „Aspect,“ 62. 
Diese Nuance der Aspektualität ist im Griechischen durch $\rceil \eta \tau \varepsilon \dot{\varepsilon} \omega$ als Modalverb (+ Inf. $)^{99}$ auch lexikalisch realisiert. ${ }^{100}$

Iterative Aspektnuancierung, also die Darstellung einer Aktserie (,die wiederholte Verwirklichung des Verbinhalts“) ${ }^{101}$ ergibt sich hingegen, wenn im Griechischen eine Verbkonstellation mit nicht-durativem Situationstyp im Durativ erscheint. Thomson führt etwa die englische Verbkonstellation [the light flash] an (ein „semelfactive“), ${ }^{102}$ die grundsätzlich als einzelnes Aufleuchten oder als Serie von Blitzen verstanden werden kann: Der imperfektive (bzw. im Englischen: progressive) Aspekt kann im Normalfall nicht für ein Einzelereignis verwendet werden, da es nicht möglich ist, eine Betrachtzeit zu finden - egal wie engmaschig sie gewählt wird - die nicht auch den Situationsendpunkt beinhalten würde. Entsprechend muss sich ein Satz wie „The light was flashing.“ auf ein wiederholtes Geschehen beziehen, wie die folgende Darstellung Abb. 33 demonstriert: ${ }^{103}$

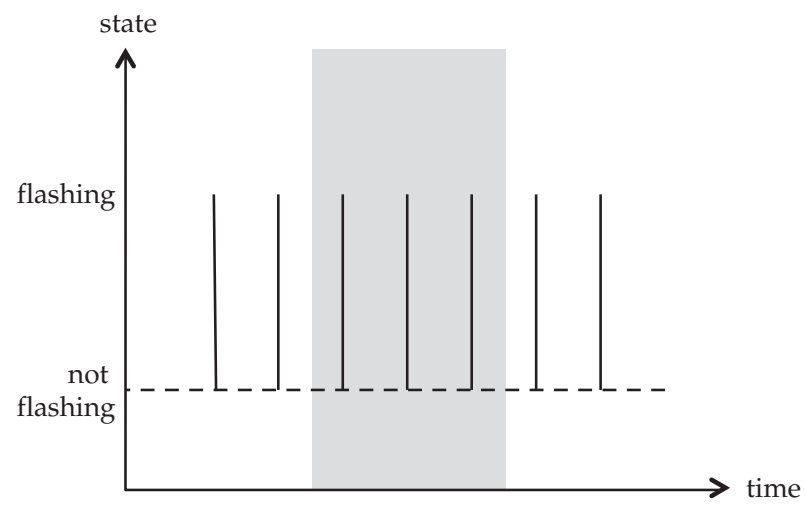

Abb. 33: Verbkonstellation mit punktuellem Aktionsartpotential im Durativ.

Die griechische Aspektunterscheidung liefert in diesem Punkt also einen durchaus wichtigen Beitrag zur Charakterisierung der grammatischen Textstruktur narrativer Texte: Der Aspekt kann (im Zusammenhang mit bestimmten Situationstypen) verwendet werden, um das in einer Proposition ausgedrückte Vorgangskonzept

99 Vgl. mit îva 1. Kor 14,12.

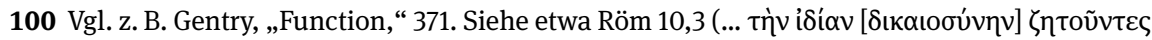

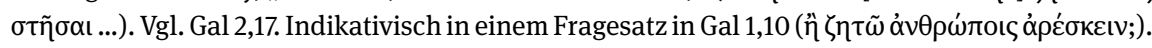
101 GGNT 194c.

102 Analoges gilt für „achievements.“ Vgl. hierzu GGNT 194n mit Verweis auf Apg 7,11: [oi

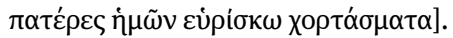

103 Thomson, „Aspect,“ 65. 
als mehrfach realisiert darzustellen. Das Griechische verfügt damit über eine grammatische Möglichkeit, um einen Verbinhalt als Ereignisbündel ${ }^{104}$ auszudrücken. Auch eine einzelne Verbform kann folglich mehrere Einzelereignisse zum Ausdruck bringen - und damit die Erfordernisse einer Minimalerzählung erfüllen.

Im Fall von ,achievement“ mit „preface“ („climaxes“) ist die Situation etwas anders gelagert. Auch hier ist der Situationstyp grundsätzlich ja punktuell, sodass die Betrachtungszeit nicht kleiner als die Ereigniszeit sein kann. Der Durativ ist daher in diesen Fällen auf das „preface“ bezogen und führt so zu einer Transformation des Situationstyps zu einem nicht-punktuellen „accomplishment,“ welches Dauer aufweist und somit auch einen Endpunkt, der gemäß der Aspektbedeutung dann wieder ausgeschlossen werden kann, sodass sich eine lineare Aspektnuancierung und somit eine Aktivität ergibt. Thomson stellt das Gemeinte

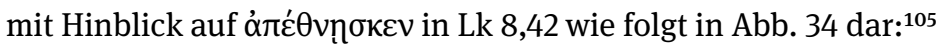

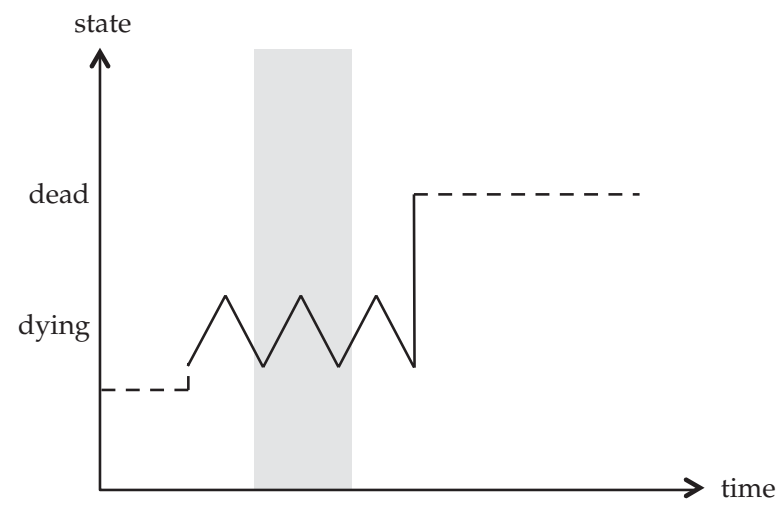

Abb. 34: „Climaxes“ im Durativ.

\subsubsection{Aorist}

Der Aorist bringt den „Verbinhalt schlechthin“ zum Ausdruck. ${ }^{106}$ Er wird in der Regel mit der Metapher der „Außenperspektive“ verbunden, womit gemeint ist, dass die Situation zeitlich begrenzt (das Ganze von außen betrachtet) wahrgenommen/dargestellt wird. ${ }^{107}$ Sprachübergreifend wird ein solcher Aspekt als ,perfektiv“ (in Opposition zum „imperfektiven“ Aspekt) bezeichnet, was in Arbeiten zum

104 Siehe zu diesem ,iterativen Erzählen“ oben, Kapitel 3, Abschnitt 4.2.

105 Thomson, „Aspect,“ 66.

106 GGNT 194e.

107 GGNT 192c. Vgl. Duden 569. 
Neuen Testament aber aufgrund der etablierten Rede vom Perfekt teilweise unterlassen wird. ${ }^{108}$ Der Aorist bezeichnet also „die Handlung schlechthin als geschehen und zum Abschluss gelangt."109

Heinrich von Siebenthal hält fest, der Aorist sei „der grundsätzlich unauffällige bzw. ,unmarkierte“ Aspekt. "110 Er sage anders als die beiden anderen Stämme nichts über Verlauf oder Ergebnis einer Situation aus und sei in dieser Hinsicht, seiner Etymologie entsprechend, „unbestimmt.“111 Eine Gefahr dieser Charakterisierung ist, dass zu wenig über den Beitrag ausgesagt wird, den der Aorist zur Nuancierung der kommunizierten Situation beiträgt. ${ }^{112}$ Anders als das Futur ist der Aorist nicht aspekt-neutral. ${ }^{113}$ Die Rede von der Außenperspektive und vom Verbinhalt als Ganzem impliziert entsprechend, dass auch ein etwaiger Endpunkt der Situation mit im Blick ist (bzw. nicht-metaphorisch: mit ausgedrückt wird). Im aoristischen Aspekt ist also die Betrachtzeit identisch mit der Ereigniszeit - und beinhaltet dementsprechend auch einen eventuell vorliegenden Endpunkt. ${ }^{114}$

Wie interagieren telische Aktionsart und aoristischer Aspekt? Die folgende Abbildung von Thomson ${ }^{115}$ (Abb. 35) kann als Darstellung der Handlung [S rennen einen Kilometer] verstanden werden. ${ }^{116}$

Ein mit perfektivem Aspekt formulierter Satz der Verbkonstellation [S rennen einen Kilometer] kann daher auch nur dann wahr sein, wenn der Läufer die volle

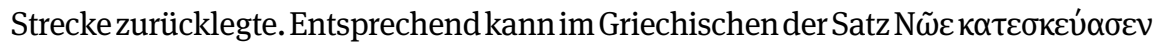

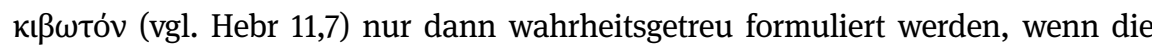
Konstruktion (zumindest im Rahmen der erzählten Welt) auch zum Abschluss kam.

Meist wird hier zwischen einer konstatierend-komplexiven Nuance des Aspekts auf der einen Seite und einem effektiven (bzw. „resultativen“/,perfektiven“) Gebrauch

108 CGCG 33.6 beschreitet einen Mittelweg und spricht vom Aoriststamm aber vom perfektiven Aspekt.

109 KG 386.

110 GGNT 194e.

111 GGNT 194e mit Verweis auf BR 77.

112 Vgl. Wallace, Grammar, 500. Der Aorist ist im Vergleich zum imperfektiven Aspekt eben doch für temporale Begrenztheit markiert. Vgl. auch die sehr hilfreichen Anmerkungen von Wilson und Aubrey, „Markedness“ zu verschiedenen - nicht immer kompatiblen - Konzepten von Markiertheit. 113 Vgl. Thomson, „Aspect,“ 60 zu diesem Konsensus. Die Rede von der fehlenden Codierung für „Andauern ... oder Nichtandauern“ (GGNT 194e) könnte hier missverstanden werden. Gemeint ist jedoch wohl nicht das Erreichen/Nichterreichen des zeitlichen Endpunktes der Situation, sondern der Fokus auf Linearität (vgl. GGNT 194b) im Durativ.

114 Thomson, „Aspect,“ 60.

115 Thomson, „Aspect,“ 60.

116 Es handelt sich dabei um ein „accomplishment“ bzw. um ein „active accomplishment“ (eine Aktivität mit Zielpunkt). 


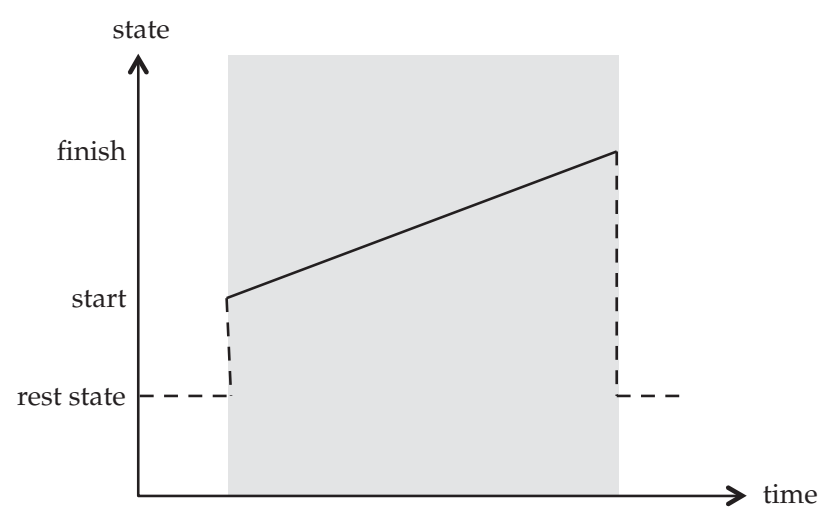

Abb. 35: Verbkonstellation mit durativem und telischen Aktionsartpotential im Aorist.

unterschieden: Im ersten Fall wird schlicht die „Verwirklichung des Verbinhalts“ ausgesagt, ${ }^{117}$ im letzteren steht der Endpunkt des zielorientierten Geschehens im Fokus. ${ }^{118}$ KG schreiben, dass „,der Aorist ... als Form der abgeschlossenen Handlung die Erreichung des Ziels nachdrücklich hervor[hebt]." ${ }^{119}$ Bei Heinrich von Siebenthal handelt es sich um eine durch den Verbinhalt ermöglichte, letztlich durch den Kontext wahrscheinlich gemachte Fokussierung. ${ }^{120}$

Bei atelischen Verbkonstellationen ${ }^{121}$ stellt hingegen der Anfangspunkt die einzige innerhalb der Betrachtzeit auszumachende Begrenzung (,boundary“) der Situation dar. Entsprechend liegt hier auch häufig eine auf diesen Punkt fokussierende ingressive Verwendung des Aorists vor. ${ }^{122}$ Auch hier sieht Heinrich von Siebenthal eine kontextuell bedingte Fokussierung gegenüber dem mit derselben Verbkonstellation ebenso möglichen konstatierend-komplexiven Gebrauchsweise. ${ }^{123}$ Während allerdings beim effektiven Aorist ein in der telischen Situation enthaltener Endpunkt schlicht hervorgehoben wird, geht der ingressive Gebrauch des Aorists mit einer Transformation des Situationstyps einher. Es steht also

117 GGNT $194 \mathrm{f}$.

118 GGNT 194j.

119 KG 386e.

120 GGNT 195 f.

121 Vgl. GGNT 194h und CGCG 33.29.

122 Vgl. GGNT 194i. Vgl. KG 386e (Hervorhebung nicht im Original): „Bei Verben, deren Präsens einen dauernden Zustand oder eine fortgesetzte Handlung bezeichnet, ist der Aorist, und zwar nicht bloss im Indikativ, sondern auch in allen übrigen Formen, oft durch Wendungen zu übersetzen, die das Eintreten in diesen Zustand, den Beginn der Handlung ausdrücken.“

123 GGNT 195e. 
nicht einfach der Anfangspunkt eines Zustands „im Fokus.“124 Vielmehr wird der Übergang zwischen zwei Zuständen selbst betrachtet - und dieser Übergang ist (anders als der erreichte Zustand) telisch.

Die Deckungsgleichheit von Betrachtzeit und Ereigniszeit erlaubt, dass im Aorist auch Einzel-Situationen zum Ausdruck gebracht werden können, die als punktuell gelten, also nicht weiter in Phasen zerteilt werden können, sodass eine Betrachtzeit gefunden werden könnte, die einen Endpunkt des Geschehens nicht enthielte. Während, wie oben gezeigt, im Durativ ein iteratives Verständnis von ,semelfactives“ bzw. ein den Situationstyp transformierendes lineares Verständnis von ,achievements with prefaces“ notwendig ist, kann der Aorist den Moment der Realisierung des Verbgeschehens selbst umfassen, wie Abb. 36 und Abb. 37 verdeutlichen. ${ }^{125}$

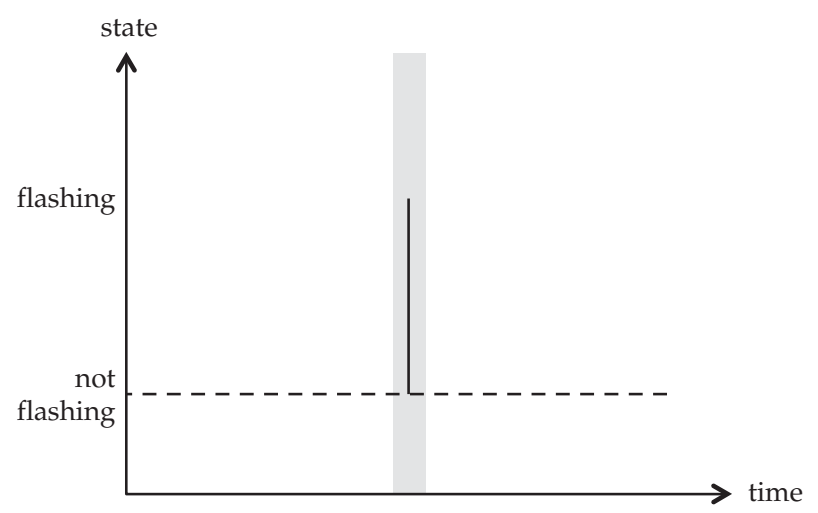

Abb. 36: „Semelfactives“ im Aorist (nicht-komplexiv).

Der Aorist eignet sich also, um Verben, die „von Natur aus (Aktionsart) einen einzigen Akt mit kaum wahrnehmbarer zeitlicher Ausdehnung bezeichnen“ ohne weitere Nuancierung zum Ausdruck zu bringen. ${ }^{126}$ Zugleich - und laut Heinrich von Siebenthal „nicht weniger häufig“ - kann der Aorist konstatierend-komplexiv auch Serien von Situationen zusammenfassen, sodass „der Redende mit dem Aoriste die ganze Handlung in einen einzigen Punkt zusammendrängt." ${ }^{27}$ Es werden dann also die innerhalb der Betrachtzeit liegenden Situationen als

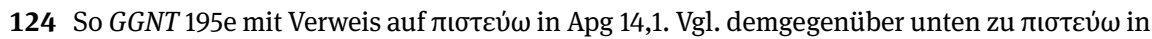
der Diskussion zum Resultativ.

125 Thomson, „Aspect,“ 65-66.

126 GGNT 194g.

127 KG 386e. Vgl. auch: Der Aorist „bezeichnet ... an sich nicht die momentane [d. h. „punktuelle“] Handlung im gewöhnlichen Sinne, sondern jede vergangene Handlung auch von der längsten Dauer wird durch den Aorist ausgedrückt, wenn sie einfach als geschehen konstatiert wird.“ 


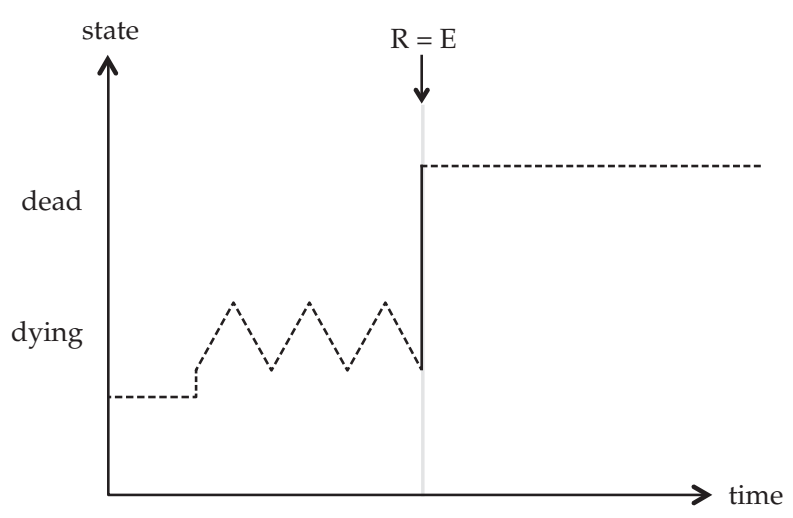

Abb. 37: „Climaxes“ im Aorist (nicht-komplexiv).

Einheit betrachtet/dargestellt (,an entire period ... viewed as a complete whole from beginning to end, without any interest in its component parts“). ${ }^{128}$

\subsubsection{Resultativ}

\subsubsection{Eine vorläufige Definition}

Laut Heinrich von Siebenthal kennzeichnet der Resultativ „ein Geschehen als etwas im Ergebnis Vorliegendes.“129 Andere betonen die „gegenwärtige Relevanz“ als die grundlegendste Eigenschaft. ${ }^{130}$ Wie schwierig dieser Aspekt zu fassen ist, zeigt die heterogene Charakterisierung in der CGCG: ${ }^{131}$,[It] presents an action as a state resulting from a preceding completed action, or it signifies that the effects of the completed action are somehow still relevant.“

Über die Relation von Betrachtzeit und Ereigniszeit lässt sich allerdings auch hier zumindest eine vorläufige Definition aufstellen: resultativer Aspekt liegt dementsprechend vor, wenn die Gesamtheit der Ereigniszeit der Betrachtzeit vorausgeht. ${ }^{132}$ Mit diesem Verständnis des resultativen Aspekts lassen sich viele Konsequenzen für die Aspektnuancierung nachvollziehen. Es treten jedoch auch Probleme auf, die eine Konretisierung erfordern. Die Problematik und eine von Crellin gebotene Lösungsmöglichkeit werden im Folgenden grob skizziert.

128 CGCG 33.30.

129 GGNT 194k.

130 Allan, „Tense,“113: „current relevance.“

131 CGCG 33.6.

132 Thomson, „Aspect,“ 36. 


\subsubsection{Der Resultativ mit transformativen Verben}

Sehr gut lassen die Unterschiede zwischen den Aspekten mit Bezug auf diese Definition anhand von „achievements with preface“ verdeutlichen, also dem

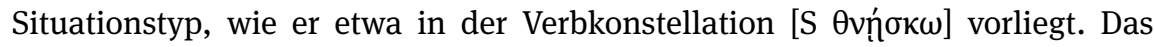
eigentliche Sterben ist punktuell, doch geht dem (meist) auch ein Prozess des Sterbens voraus. ${ }^{133}$ Im Aorist kann tatsächlich der (a) Moment des Umschlagens vom Zustand des Lebens zum Zustand des Totseins betrachtet werden. Der durative Aspekt erfordert einen Fokus auf das (b) „preface,“ also den zum tatsächlichen Todeszeitpunkt führenden Prozess. Im Resultativ ist demgegenüber (z. B.

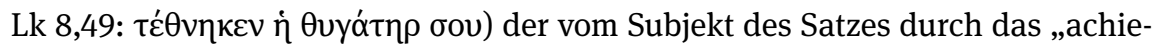
vement“ erreichte (c) Zustand im Blick, wie dies Abb. 39 illustriert: ${ }^{134}$

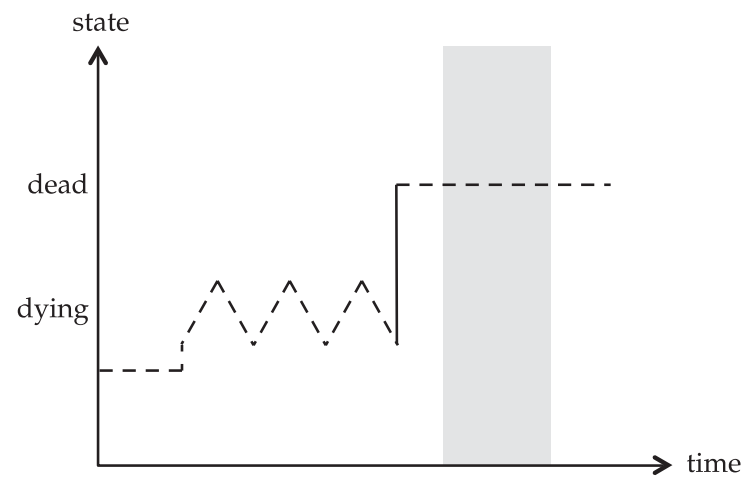

Abb. 38: „Climax“ im Resultativ.

[S $\theta v \eta n ́ \sigma \kappa \omega]$ ist ein intransitives Verb, die darin ausgedrückte Situation involviert lediglich das Subjekt. Es kann daher im Resultativ auch nur der vom Subjekt erreichte Zustand im Blick sein. Anders gesagt: Bei intransitiven Verben wie

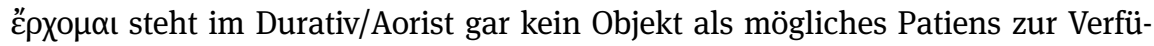
gung, auf das in der Betrachtzeit fokussiert werden könnte. Abb. 39 zeigt, wie sich intransitive Transformationsverben in den verschiedenen Aspekten verhalten.

\begin{tabular}{|c|c|c|c|c|}
\hline & Subjekt & Prädikat & Lokale Angabe/Ergänzung & Akkusativobjekt \\
\hline Aorist & 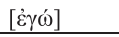 & $\tilde{\eta} \lambda \theta$ ov & 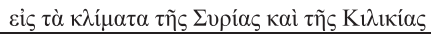 & - \\
\hline Durativ & 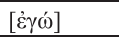 & $\varepsilon \dddot{\rho} \chi \rho \mu \alpha 1$ & $\pi \rho \grave{s} \varsigma \dot{v} \mu \tilde{\alpha} \varsigma$ & - \\
\hline Resultativ & $\tau \grave{\alpha} \kappa \alpha \tau^{\prime} \dot{\varepsilon} \mu \grave{\varepsilon}$ & $\dot{\varepsilon} \lambda \dot{\eta} \lambda v \theta \varepsilon v$ & 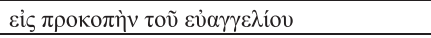 & - \\
\hline
\end{tabular}

Abb. 39: Intransitive transformative Verben in den verschiedenen Aspekt-Stämmen (Aorist nach Gal 1,21, Durativ nach 2. Kor 13,1, Resultativ nach Phil 1,12).

133 Thomson, „Aspect,“ 59.

134 Thomson, „Aspect,“ 68. 
Dieser Gebrauch des Resultativs mit Verbkonstellationen, welche einen resultierenden Zustand für das Subjekt involvieren, stellt die frühste Phase der sehr umstrittenen Entwicklung des Aspekts dar. ${ }^{135}$

Grundsätzlich bleibt der resultative Aspekt überwiegend transformativen Verben vorbehalten, wobei sich der Gebrauch entlang des Grads an Transitivität der Verbkonstellation ausdifferenziert. ${ }^{136}$ In prototypisch-transitiven Verbkonstellationen sind (a) Subjekt und Objekt maximal von einander unterschieden (anders als im Satz „Ich wasche meine Hände.“), (b) bringt das als Agens auftretende Subjekt die Situation willentlich voran (vgl. [S anschauen AkkO] vs. [S sehen AkkO]) und (c) ist lediglich das als Patiens auftretende Objekt betroffen. ${ }^{137}$

Verben mit niedriger Transitivität interagieren ähnlich mit dem Resultativ wie das im Fall der schon besprochenen intransitiven Verben der Fall ist. Zwar hat das Verb auch im Resultativ ein Akkusativobjekt, doch ist es die Situation des Subjekts, welche im Zentrum steht. Ein Beispiel wäre etwa $\mu \alpha v \theta \alpha \dot{\alpha} \omega \omega$, wobei hier das Gelernte in seinem ursprünglichen Zustand bleibt, der Lernende jedoch einen Zustandwechsel erreicht, also - entgegen dem prototypischen Fall von Transitivität - das Patiens auf das Agens einwirkt. Betrachtet wird folglich dieser erreichte Zustand des Gelernthabens = Wissens. Die folgende Übersicht in Abb. 40 stellt die verschiedenen Konstruktionen vergleichend dar:

\begin{tabular}{|c|c|c|c|}
\hline & Subjekt & Prädikat & Akkusativobjekt \\
\hline Aorist & $\dot{\varepsilon} \gamma \grave{\omega}$ & $\varepsilon \ddot{\mu} \mu \alpha \theta \mathrm{ov}$ & 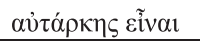 \\
\hline Durativ & {$[\alpha \tilde{v} \tau \alpha 1]$} & $\mu \alpha v \theta \alpha ́ v o v \sigma \mathrm{I} v$ & $\dot{\alpha} \rho \gamma \alpha \hat{~[\varepsilon \tilde{v} v \alpha 1]}$ \\
\hline Resultativ & $\begin{array}{l}\dot{\eta} \gamma \lambda \tilde{\omega} \sigma \sigma \alpha \\
\alpha \tilde{v} \tau \tilde{\omega} \nu\end{array}$ & $\mu \varepsilon \mu \alpha \dot{\theta} \theta \eta \kappa \varepsilon v$ & $\lambda \alpha \lambda \varepsilon \tilde{i} v \psi \varepsilon v \delta \delta \tilde{\eta}$ \\
\hline
\end{tabular}

Abb. 40: Verben mit geringer Transitivität in den verschiedenen Aspekt-Stämmen (Aorist nach Phil 4,11, Durativ nach 1. Tim 5,13, Resultativ nach Jer 9,4).

Einen mittleren Grad an Transitivität weisen Verbkonstellationen auf, die nicht nur ein Akkusativobjekt beinhalten, sondern wo darüber hinaus sowohl Agens als auch Patiens von der Handlung betroffen sind. Aubrey differenziert hier zwischen einem Einfluss der Handlung auf die physische (Verben der verursachten

135 Vgl. Allan, „Tense,“ 100-114 zu den verschiedenen Stufen der Entwicklung. Auf umstrittene Entwicklungen wird im Laufe der Diskussion jeweils nur kurz verwiesen werden.

136 Aubrey, „Perfect Tense.“

137 Aubrey, „Perfect Tense“ folgt hier Næss, Prototypical Transitivity, 33-36. Siehe auch unten, Abschnitt 4 im Kontext der Diskussion der Diathese zu verschiedenen (aoristischen) MediumFormen und ihrem Verhältnis zum transitiven Prototyp. 
Bewegung, der Übertragung, der Produktion, des Erreichens etc.) und die kognitive Sphäre (Verben der Kognition und Kommunikation), wobei sich die entsprechende Resultativ-Form gleich verhält. ${ }^{138}$

Auch hier findet keine Reduktion der an der Situation Beteiligten statt. Agens und Patiens erscheinen beide auch in der Formulierung im Resultativ, wie folgendes Beispiel in Abb. 41 zeigt:139

\begin{tabular}{|c|c|c|c|}
\hline & Subjekt & Prädikat & Akkusativobjekt \\
\hline Aorist & 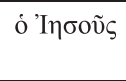 & $\dot{\varepsilon} \tau \varepsilon ́ \lambda \varepsilon \sigma \varepsilon V$ & 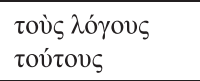 \\
\hline Durativ & {$[\dot{0} \mu \varepsilon \bar{\varsigma} \zeta]$} & $\tau \varepsilon \lambda \varepsilon i \tau \varepsilon \varepsilon$ & بópous \\
\hline Resultativ & {$[\dot{\varepsilon} \gamma \omega \dot{\omega}]$} & $\tau \varepsilon \tau \varepsilon \dot{\lambda} \varepsilon \varepsilon \kappa \alpha$ & 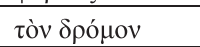 \\
\hline
\end{tabular}

Abb. 41: Verben mit mittlerer Transitivität in den verschiedenen Aspekt-Stämmen (Aorist nach Mt 7,28, Durativ nach Röm 13,6, ${ }^{140}$ Resultativ nach 2. Tim 4,7).

Aubrey meint, hier könne sowohl der „Resultat-Zustand“141 als auch die vorausgehende „Ereignis-Vollendung“142 im Blick sein. Auch Heinrich von Siebenthal meint zum Ind. Perf., es trete „neben dem gegenwärtigen Zustand auch das dazu führende vergangene Geschehen (mehr oder weniger deutlich) ins Blickfeld.“ In der obigen Definition nach Thomson müsste man einen solchen Fokus vermutlich durch eine Rückverschiebung der Betrachtzeit erklären, sodass auch das Ende der Situationszeit von ihr erfasst wird. ${ }^{143}$

138 Antike Konzeptionen (vgl. z. B. Heilig, Paul’s Triumph, 182-184) mögen hier in der Tat unterschiedliche Zuordnungen vorgenommen haben, es ergeben sich allerdings in der Verwendung des Resultativs anscheinend keine Unterschiede, da beide Fälle gleich mit dem Aspekt interagieren.

139 Aubrey, „Perfect Tense“ merkt an, dass bei Verben der Kommunikation das Akkusativobjekt fehlen kann: „This is a natural result of the fact that the perfect is being used immediately after finishing communicating the content.“

140 Die Semantik ist hier anders, fällt aber auch in die große Kategorie der physischen Betrof-

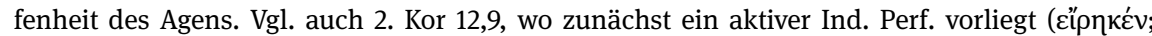
die Funktion des Ind. Perf. ist hier sehr umstritten, siehe dazu unten, Abschnitt 3.3.2.2, Abschnitt 3.3.2.3 und Abschnitt 3.5.), auf den dann aber im mediopassiven Ind. Präs. diese Aussage

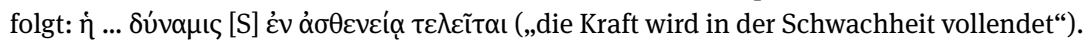

141 Aubrey, „Perfect Tense,“: „result-state.“

142 Aubrey, „Greek Perfect“: „Event-Completion.“

143 Siehe Crellin, „Semantics,“ 453, der hierin die Ursache für die Verschmelzung mit dem Ind. Aor. sieht. 
Ob sich die Betrachtung der resultierenden Situation an Agens oder Patiens orientiert, ist nach Aubrey rein kontextuell zu erschließen. Er verweist auf Joh 17,22, wo Gott als „Geber“ hervorgehoben scheint, in Joh 19,22 sei dementsprechend das „Geschriebene“ zentral.

$\mathrm{Zu}$ einer Reduktion der Beteiligten kommt es allerdings beim hier möglichen medialen Gebrauch des Stammes, wobei sich dann der Fokus vom Abschluss des Ereignisses zum Resultat, ohne Berücksichtigung des Vorangehenden, ver-

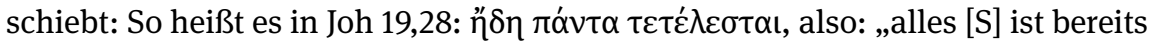
vollendet.“

Bei Verben mit hoher Transitivität stellt sich die Situation anders dar: Im Durativ und Aorist tritt das Subjekt als Agens und das Akkusativobjekt als Patiens auf, wobei lediglich das Patiens von der Handlung betroffen ist. Im Resultativ kommt es dann zu einer Reduktion der Beteiligten: Subjekt der intransitiven ${ }^{144}$ resultativen Verbform ist nun das von der Handlung im Aorist/Durativ betroffene Patiens.

Die folgende Gegenüberstellung in Abb. 42 von aoristischer und resultativer Verbkonstellation mit ávoíy $\omega$ zeigt dies:

\begin{tabular}{|c|c|c|c|c|}
\hline & Subjekt & Prädikat & Dativobjekt & Akkusativobjekt \\
\hline Aorist & 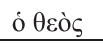 & 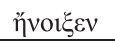 & 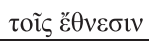 & $\theta \dot{v} \rho \alpha v$ \\
\hline Resultativ & $\theta \dot{v} \rho \alpha$ & 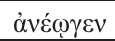 & $\mu \mathrm{ol}$ & - \\
\hline
\end{tabular}

Abb. 42: Verben mit hoher Transitivität im Aorist (nach Apg 14,27) und Resultativ (nach 1. Kor 16,9).

Dasselbe Muster liegt auch der folgenden Abb. 43 zugrunde, wobei dieser Fall bereits dadurch von prototypischer Transitivität abweicht, dass das im Aorist und Durativ betroffene Patiens Teil des Agens ist (,ihr/mein/unser Mund“):

\begin{tabular}{|c|c|c|c|}
\hline & Subjekt & Prädikat & Akkusativobjekt \\
\hline Aorist & $\dot{\eta} \gamma \tilde{\eta}$ & $\eta \dddot{n} v 01 \xi \varepsilon v$ & $\tau o ̀ ~ \sigma \tau o ́ \mu \alpha \alpha v ่ \tau \tilde{\eta} \varsigma$ \\
\hline Durativ & $\mathrm{I} \omega \beta$ & àvoízદl & $\tau o ̀ ~ \sigma \tau o ́ \mu \alpha \alpha \dot{v} \tau o \tilde{v}$ \\
\hline Resultativ & 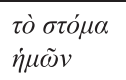 & 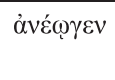 & - \\
\hline
\end{tabular}

Abb. 43: Verben mit betroffenem Patiens als Teil des Agens (Aorist nach Offb 12,16, Durativ nach ljob 35,16, Resultativ nach 2. Kor 6,11).

144 Entsprechend bilden manche Verben (z. B. í $\sigma \tau \eta \mathrm{\iota})$ ausschließlich aktive Resultativformen, andere (z. B. $\kappa \lambda \alpha \dot{\alpha} \omega)$ ausschließlich mediale. 


\subsubsection{Der Resultativ mit Zustandsverben}

Mit transformativen Verben, die naturgemäß einen Zustandswechsel beinhalten, lässt sich die Funktion des resultativen Aspekts also gut anhand verschiedener Grade an Transitivität nachvollziehen. Mit Verbkonstellationen, die selbst bereits einen reinen Zustand ausdrücken, verträgt sich der Resultativ hingegen nur schwer: „Thus far, we have seen that the concepts of event completion and result state are integral to the perfect's usage. Yet, the basic idea of stativity is contrary to the very concepts of completion \& result." ${ }^{145}$ Vor dem Hintergrund der obigen Definition des resultativen Aspekts nach Thomson lässt sich das Problem analog beschreiben als das Fehlen einer Ereigniszeit, die vor Einsetzen der Betrachtzeit zu einem Ende kommen würde.

Eine erste Option ${ }^{146}$ besteht in der für das fragliche Verb geschehenden Bildung eines Situationstyps, der einen Eintritt in einen Zustand beinhaltet. Die

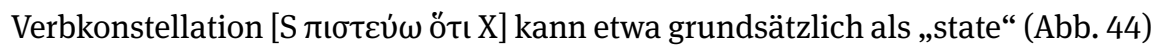
und als „achievement“ (Abb. 45) verstanden werden. ${ }^{147}$ Der Gebrauch des resultativen Aspekts kann dementsprechend als deutlicher Hinweis darauf verstanden werden, dass der Situationstyp ,achievement“ zugrunde liegt: ${ }^{148}$

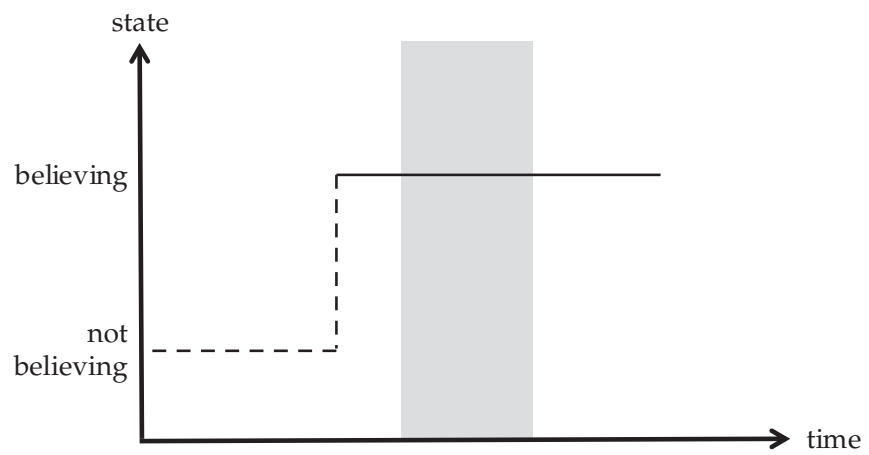

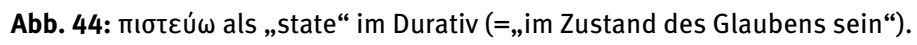

145 Aubrey, „Greek Perfect.“ Großschreibung in kursive Hervorhebung umgewandelt.

146 Es ist die einzige bei Thomson, „Aspect“ diskutierte Option. Aubrey, „Greek Perfect“ führt sie wie folgt ein: „A speaker can convert the state and retain the change-of-state semantics.“ Bei Crellin, „Semantics“ erscheint sie als grundsätzliche (für die diskutierten Verben jedoch abgelehnte) Option in zwei (duplizierten) Fußnoten auf S. 432 und 440.

147 Thomson, „Aspect,“ 53 und 57, wobei er im ersten Fall eine umfassendere Verbkonstellation identifiziert.

148 Thomson, „Aspect,“ 53 (modifiziert) und 68. 


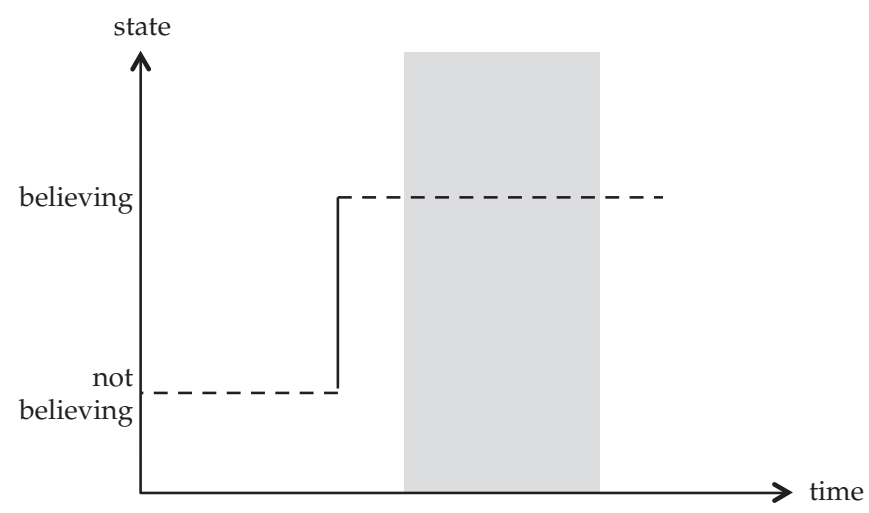

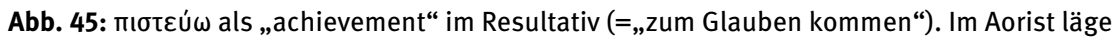
derselbe Situationstyp vor, aber eine andere Betrachtzeit.

Es gibt allerdings durchaus Fälle, in welchen der Resultativ mit Verbkonstellationen verbunden wird, welche Zustände zum Ausdruck bringen, wobei dann der Zustand ,concurrent with the reference time of the clause with no reference to any prior event“ ist. ${ }^{149}$ Ein Beispiel hierfür wäre die Verbkonstellation [S $\dot{\varepsilon} \lambda \pi i \zeta \omega$

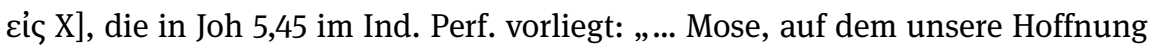

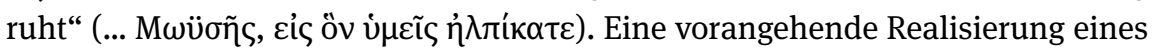
Ereignisses scheint nicht im Blick. ${ }^{150}$ Die Bezeichnung des Aspekts als „Resultativ" ist hier in der Tat suboptimal. ${ }^{151}$

149 Crellin, „Semantics,“ 432.

150 Vgl. GGNT 194k: ,... der gemeinte Zustand [steht] so stark im Fokus ..., dass ein allenfalls dazu führendes Geschehen so gut wie ausgeblendet bleibt.“ Vgl. CGCG 33.36 zu Fällen mit und ohne (z. B. oĩ $\delta \alpha)$ korrespondierendem Durativ-Stamm. Siehe auch KG 384.

151 Andere Grammatiken sprechen schlicht vom „perfect stem“ und entsprechend auch vom „perfect-stem aspect“ (CGCG 33.6 und 33.12). Manche Autoren bevorzugen die sich noch weiter von herkömmlicher Terminologie abhebende Bezeichnung als „,combinative aspect.“ Siehe dazu Ellis, „Aspect-Prominence,“ 142-143 (,reflecting the perfective nature of the verbal event and the imperfective nature of its ongoing relevance”) und Buth, „Morphology,“ 423 („So I will stay with the abstract analysis of the Greek perfect as \{+ perfective, + imperfective $\}$ as long as one allows the definition of ,imperfective“ to be a subset definition of ,continuing relevance.“”). Der Resultativ ist somit auch nicht grundsätzlich „statisch“ - vielmehr ergibt sich diese Nuance aus dem Zusammenspiel mit dem Situationstyp, „the stative effect depends on the procedural character of the verb in question“ (Thomson, „Aspect,“ 69). Siehe dazu unten zu Fällen, in welchen der Situationstyp keinen Zustand für das Subjekt anzeigt. Hingegen erscheint „retrospective“ (vgl. Thomson, „Aspect,“ 67) unangebracht in Fällen, in welchen ein Zustand in der Verbkonstellation zum Ausdruck kommt und überhaupt nicht „zurück“ geschaut wird. 
Um dieses Problem zu lösen, fokussiert Crellin in seinem Definitionsversuch auf die Zeit nach dem Ereignis/der Situation, die „Situations-Nachzeit,“152 in welche die Betrachtungszeit „properly included“ ist. ${ }^{153}$ Er räumt zunächst ein, dass es im Fall von Zuständen oft schwierig sei, einen Endpunkt der Situation zu identifizieren, nach welchem die Betrachtzeit angesetzt werden könnte. ${ }^{154}$ Crellin stellt daher in einem weiteren Schritt die Gemeinsamkeiten von reinen Zuständen, Resultatszuständen, ,and the situation pertaining after an event" heraus. ${ }^{155}$ Ihnen sei gemeinsam, dass sie homogen und atelisch seien. Er nimmt statt einer Situations-Nachzeit daher nun eine Situationszeit an, in welche die Betrachtzeit eingebettet sei. ${ }^{156}$ Für diese werde im Resultativ „a homogeneous atelic eventuality“ - also eine homogene und atelische Situation ${ }^{157}$ - vom Prädikat für das grammatische Subjekt abgeleitet. ${ }^{158}$ Im Fall von Verbkonstellationen, die für das Subjekt keinen Zustand mit sich bringen, gelte daher: „the only means that the perfect has of deriving a homogeneous atelic eventuality from the predicate is by asserting that a participant property holds at TTop [Betrachtzeit], namely the property of having once done the event described by the predicate." 159

Auf diese Weise lässt sich nun die Aspektnuancierung der verschiedenen Situationstypen durch den Resultativ vollständig erfassen: ${ }^{160}$ Bei Verbkonstellationen, welche (I) eine Zuständsänderung des Subjekts beinhalten, greift der Resultativ für die Situationszeit (die Situations-Nachzeit des ursprünglichen Definitionsversuches) diesen resultativen Zustand auf und integriert die Betrachtzeit in

152 Crellin, „Semantics,“ 442: „For any event there exists a set of times after the event itself has terminated, the posttime of that event,“ die „TPostSit.“ Mit Bezug auf Klein, „Present Perfect Puzzle."

153 Crellin, „Semantics,“ 452.

154 Crellin, „Semantics,“ 449.

155 Crellin, „Semantics,“ 449.

156 Crellin, „Semantics,“ 451.

157 Breindl, „Grundbegriffe,“ 122 hält die Eindeutschung „Eventualität“ für „unglücklich.“ Wir bleiben daher bei „Situation.“

158 Crellin, „Semantics,“ 451. Hervorhebung nicht im Original.

159 Crellin, „Semantics,“ 451. Siehe auch Crellin, Syntax and Semantics, etwa S. 10 für Beispiele außerhalb des NT für den Resultativ „describing a no longer existing entity.“

160 Vgl. die Zusammenfassung bei Crellin, „Semantics,“ 454: „Where a predicate itself describes a state for the subject (state predicates and change of state predicates), the perfect may simply return this state. By contrast, where a predicate does not describe or give rise to a state for the subject, the perfect derives a homogeneous atelic eventuality for the subject, a property of the subject based on the subject having previously participated in the event described by the predicate." Vgl. auch die Wiedergabe mit englischen Beispielen bei Horrocks, „Envoi,“ 633. 
diese Situationszeit. Im Indikativ wird dann zusätzlich die Orientierungszeit als von der Betrachtzeit umfasst spezifiziert. ${ }^{161}$ Dies ist etwa der Fall bei der indikati-

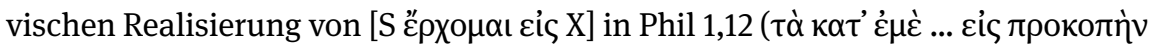

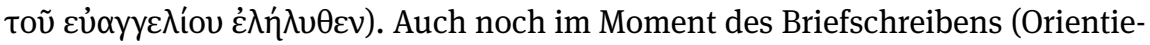
rungszeit) gilt ein Zustand, der in der Vergangenheit erreicht wurde. ${ }^{162}$ Vergleich-

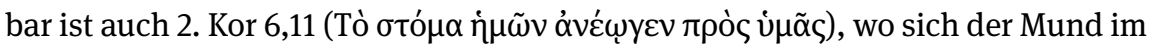
geöffneten Zustand befindet. ${ }^{163}$

Im Fall (II) des Situationstyps Zustand ist eine Möglichkeit (a), dass für die Betrachtzeit schlicht der im Prädikat enthaltene Zustand selbst herangezogen wird: So ist in Röm 5,2 das „Haben“ des Zugangs zu Gott der Zustand, welcher

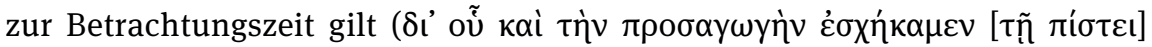

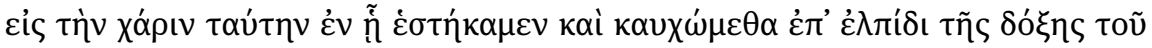
$\theta \varepsilon o \tilde{v})$. So ist nun auch Joh 5,45 sinnvoll zu verstehen und die identische Verb-

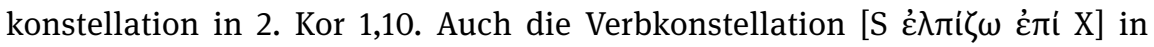

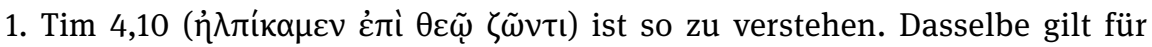
die auf Gott hoffende Witwe in 5,5, wobei hier interessanterweise direkt im nächsten Vers mit $\tau \dot{\varepsilon} \theta v \eta \kappa \varepsilon v$ der zuvor genannte Fall einer Situation mit neuem Zustand für das Subjekt vorliegt.

Auch Aubrey diskutiert letztlich diese Option: „A speaker can ... convert the change-of-state semantics and retain the stativity of the verb. "164 Dabei definiert er jedoch spezifischer Crellins „homogeneous atelic eventuality“ als ein der Intensität nach gesteigerter Grad des Zustands. ${ }^{165}$

Nach Crellin besteht letztlich aber auch noch die weitere Möglichkeit (b), dass „the state no longer holds at TTop, but once did, and the participant property of this state now holds at TTop." 166 Entsprechend könnte Röm 5,2 also rein theoretisch auch zum Ausdruck bringen, dass in der Gegenwart des Briefschreibens die Konstellation besteht, dass ,wir“ uns in der Situation befinden, solche zu sein, die einst Zugang zu Gott hatten. Notwendig ist ein solches Verständnis etwa in Mk 5,15, wo der ehemals Besessene zur Betrachtzeit Dämonen gehabt hatte. ${ }^{167}$

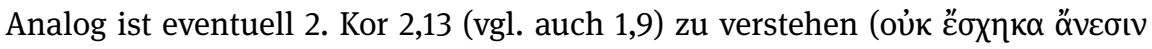

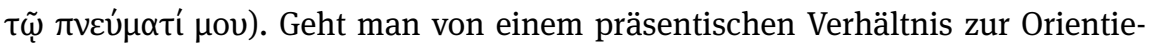

161 Vgl. Crellin, „Semantics,“ 453.

162 Vgl. Crellin, „Semantics,“ 441 mit dem Beispiel Lk 5,23. Vgl. auch 1. Thess 2,8.

$163 \mathrm{Vgl}$. Auch 1. Kor 16,9.

164 Aubrey, „Greek Perfect.“

165 Vgl. GGNT 200c zu den „Intensiv-Perfekta.“

166 Crellin, „Semantics,“ 451.

167 Crellin, „Semantics,“ 452. 
rungszeit aus, ${ }^{168}$ so muss man die Stelle dennoch nicht so verstehen, als würde Paulus für die Gegenwart aussagen, dass er keine Ruhe im Geist habe. ${ }^{169}$ Vielmehr könnte man den Vers auch so verstehen, dass Paulus hier von sich aussagt, dass für ihn in der Gegenwart gilt, dass er einer ist, der früher keine Ruhe im Geist hatte. ${ }^{170}$

\subsubsection{Die modifizierte Definition und nicht-transformative Verben}

Die letztgenannte Option gilt dann nach Crellin auch für all jene Situationstypen, welche (III) keinen Zustand (weder einen ursprünglichen, noch einen resultativen) involvieren. Hier ist die einzige homogene und atelische Eventualität der Umstand, dass das Subjekt sich in der Situation befindet, den Prädikatsinhalt bereits verwirklicht $\mathrm{zu}$ haben. Sehr instruktiv ist, was in 2. Tim 4,7

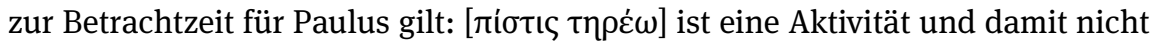

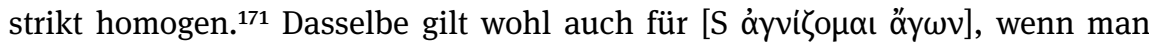
es nicht als „accomplishment“ einordnen möchte (bzw. als „active accomplis-

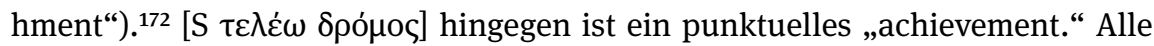
drei Verbkonstellationen involvieren keine Situation, in welcher für das Subjekt ein Zustand erreicht wird. Für die Zeit des Briefschreibens ist also schlicht ausgesagt, dass Paulus einer ist, der durch die Erfahrung reicher ist, ${ }^{173}$ „den guten Kampf gekämpft zu haben, den Lauf vollenden zu haben, den Glauben bewahrt zu

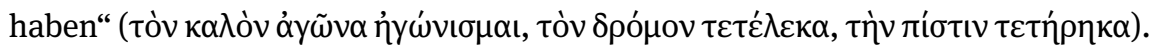

Aus Aubreys Perspektive könnte man hier allerdings kritisch anmerken, dass diese mit Akkusativobjekt konstruierten, aber nicht prototypisch transitiven Verben mit einem betroffenen Agens einhergehen, sich also durchaus sinnvoll ein resultierender Zustand identifizieren lässt, der über die bloße „participation property“ hinausgeht. ${ }^{174}$

168 Vgl. auch unten, Abschnitt 3.2.2.2 zum Ind. Perf. im späteren Narrationstyp.

169 Vgl. dazu unten, Abschnitt 3.3.2.3.

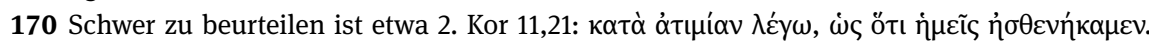
Paulus und Mitarbeiter sind zum Zeitpunkt des Schreibens solche, die entweder schwach sind oder aber die Eigenschaft mit sich tragen, zuvor schwach gewesen zu sein.

$171 \mathrm{Vgl} . \pi \alpha \rho \eta \kappa о \lambda$ oú $\because$ $\kappa \alpha \varsigma$ ([S folgen DatO]) in 1. Tim 4,6.

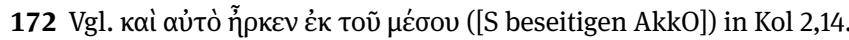

173 Vgl. Crellin, „Semantics,“ 454 zur erfahrungsbezogenen Interpretation der „property of the subject based on the subject having previously participated in the event described by the predicate.“

174 Entsprechend müsste auch die von Allan, „Tense,“ 108-112 skizzierte Phase 2 in der Entwicklung des griechischen Perfekts neu überdacht werden. Man vergleiche Aubreys Entwurf etwa mit Allan, „Tense,“ 110: „The emergence of the current relevance meaning of the perfect can be described as a form of subjectification: the objective (event-oriented) mental or physical 
Auch innerhalb von The Greek Verb Revisited bleibt Crellins Entwurf nicht gänzlich ohne Kritik. So bezieht sich Horrocks auf den Fall einer für das Subjekt nicht-transformativen Verbkonstellationen wie [S schreiben Buch]. Hier würde entsprechend für die Betrachtungszeit gelten, dass sich die durch das grammatische Subjekt repräsentierte Person in der Situation befindet, ein Buch geschrieben $\mathrm{zu}$ haben und diese Eigenschaft nun mit sich trägt. ${ }^{175}$ Horrocks merkt dazu an, dass dadurch die Rolle des Subjekts ,more passive or experiential“ uminterpretiert würde: Während in einem Satz wie „I am writing/wrote a book“ das durch das Subjekt bezeichnete Individuum klar als Agens auftritt, sei er/sie nun „primarily the carrier of a property. “176 Daraus leitet er ab, dass der Resultativ nicht auf derselben Ebene wie Aorist und Durativ anzusiedeln sei: „It is ... a facultative option that may be deployed for clarity and explicitness rather than a true third choice in the grammaticalized system of viewpoint aspect." ${ }^{177}$ Diese Einschätzung des Resultativs als Mittel der Verfeinerung bzw. Modifikation ${ }^{178}$ demonstriert den nach wie vor großen Klärungsbedarf zu dieser Thematik.

\subsection{Aspektfunktion und subjektive Aspektwahl}

\subsubsection{Aspektfunktion}

Der Aspekt stellt einen wichtigen Parameter der grammatischen Seite der Textstruktur dar. Die vorausgehende schlaglichtartige Betrachtung zeigt, wie die Kombination von Aspekt eine (teilweise recht umfassende) „Nuancierung“ des in der Verbkonstellation zum Ausdruck gebrachten Situationstyps bewirkt. Beispielsweise bewirkt die Präsentation einer eigentlich telischen Situation im durativen Aspekt eine Transformation des Situationstyps: ${ }^{179}$

[W]hile perfective representation with its focus on the boundaries of the situation is compatible with telicness, imperfective representation with its focus on the internal progression of the situation is incompatible. The result of imposing an imperfective focus on a telic situation is therefore that we conceive of the situation as a different type of situation.

property of the subject has bleached away and is replaced by the subjective (speaker-oriented) feature of current relevance.“

175 Horrocks, „Envoi,“ 633.

176 Horrocks, „Envoi,“ 634.

177 Horrocks, „Envoi,“ 634.

178 Vgl. Horrocks, „Envoi,“ 634: „The Greek perfect ... has features in common with Englishstyle refinements and modifications of aspectual character.“

179 Bache, Study, 268. 
Die neutralere Bezeichnung der „Aspektfunktion“ bietet sich an, um auch dieses Ende des Spektrums der Interaktion von Aspekt und Aktionsart zu erfassen. ${ }^{180}$

\subsubsection{Verbformen des griechischen Verbalsystems als Wahlmöglichkeiten}

Um das Erzählte einer griechisch verfassten Erzählung korrekt zu erfassen, ist ein angemessenes Verständnis der Verbsemantik und der Interaktion mit der Aspektbedeutung daher unerlässlich. Narratologisch relevant ist jedoch nicht nur, welche Situationstypen im Text zur Darstellung kommen, sondern auch, welchen Aspekt der Erzähler wählt, wo ihm tatsächlich vom Erzählten her mehrere Optionen zur Verfügung stehen. Dieses Konzept muss im Folgenden jedoch noch näher entfaltet werden.

Grundsätzlich sind es die drei Stämme, die zur Wahl stehen. Auch im Indikativ schlägt sich die Aspekt-Prominenz des griechischen Verbalsystems (in der Morphologie) nieder, wie Abb. 46 veranschaulicht: ${ }^{181}$

\begin{tabular}{|l|l|l|l|l|l|l|}
\cline { 2 - 6 } \multicolumn{2}{c|}{ Aorist-Stamm } & \multicolumn{2}{l|}{ Durativ-Stamm } & \multicolumn{2}{l|}{ Resultativ-Stamm } \\
\hline Zeitbezug & Vergangenheit & $\begin{array}{l}\text { Nicht- } \\
\text { Vergangenheit }\end{array}$ & Vergangenheit & $\begin{array}{l}\text { Nicht- } \\
\text { Vergangenheit }\end{array}$ & Vergangenheit & $\begin{array}{l}\text { Nicht- } \\
\text { Vergangenheit }\end{array}$ \\
\hline Tempus & Aorist & Futur & Imperfekt & Präsens & Plusquamperfekt & Perfekt \\
\hline
\end{tabular}

Abb. 46: Der durchgängige Einfluss des Aspekts im Indikativ.

Liegt im Text - wie bei Erzählungen üblich (siehe dazu unten, Abschnitt 3.2) eine absolute zeitliche Orientierung vor, so ergeben sich entsprechend die in Abb. 47 ersichtlichen grundsätzlichen (indikativischen) „Wahlmöglichkeiten, “ um auf die jeweilige Relation zur Sprechzeit Bezug zu nehmen:

180 Bache, „Aspect and Aktionsart,“ 67 gebraucht die Terminologie der „aspectual function“ als Gegenüber zur „pure aspectual opposition,“ auf die hier durch die Explizierung der Rede von der „Wahl“ des Aspekts eingegangen wird.

181 Angelehnt an Ellis, „Aspect-Prominence,“ 136. Vgl. Fresch, „Typology,“ 393-394 für zwei vor dem Hintergrund sprachübergreifender Untersuchungen möglicher Auflösungen des Verbsystems. Siehe AGG 202, Fußnote 50 für die Neutralisierung der Aspekt-Bedeutung im Futur. 


\begin{tabular}{|l|l|l|l|}
\cline { 2 - 3 } \multicolumn{1}{c|}{} & Vergangenheit & Gegenwart & Zukunft \\
\hline Aorist-Stamm & Aorist & -182 & Futur $^{183}$ \\
\hline Durativ-Stamm & Imperfekt & Präsens & \\
\hline Resultativ-Stamm & Plusquamperfekt & Perfekt & (Perfektfutur) $^{184}$ \\
\hline
\end{tabular}

Abb. 47: Indikativische Optionen bei vorausgesetztem Verhältnis zur Orientierungszeit.

\subsubsection{Realisierung von Durativ und Aorist bei Verben der Koine}

Zunächst ergibt sich die Möglichkeit einer Wahl zwischen verschiedenen Aspekt-Stämmen bzw. - bei absolutem Zeitbezug - verschiedenen, die Aspekte vertretenen Tempora ganz grundsätzlich aus dem bereits im Hinblick auf die Aktionsart festgehaltenen Spielraum, welcher ein Sprecher hinsichtlich der Darstellung tatsächlicher Situationen hat: ${ }^{185}$

[W]hile viewpoint aspect is indeed an essentially „temporal“ category, it is one that has little or nothing to do with the actual temporal properties of the situation described, whether in terms of its location in time or of its duration/punctuality; even momentary events, after all, may be viewed imperfectively, cf. ,he only coughed for a split second, but while he was coughing ..." It is instead a matter of the grammatically obligatory, but always subjective, choice of a speaker or writer to describe a given eventuality with either a perfective or an imperfective verb form that requires his/her audience to view that eventuality, respectively, either as „bounded“ (i. e., with a beginning and end in time, thus focusing on its completeness and by implication eliding its middle) or „unbounded“ (i. e., without a beginning or end in time, thus focusing on its incompleteness and by implication its undelimited middle).

Entsprechend weist auch die große Mehrheit der Verben sowohl einen aoristischen als auch einen durativen Stamm auf, was nach Horrocks demonstriere, dass ,these two aspectual notions ... indeed fundamentally different and very

182 KG 381: „Für die momentane Aktion existiert keine Form der Gegenwart, weil eine gegenwärtige Handlung nicht als abgeschlossen in dem oben besprochenen Sinne gelten kann, sondern stets in ihrem Verlaufe angeschaut wird.“ Vgl. 386e: „Der Indikativ des Aorists ist eine historische Zeitform, wie aus dem Augmente erhellt. Wenn für die momentane Aktion nur eine Zeitform der Vergangenheit ausgeprägt worden ist, nicht auch eine solche der Gegenwart, so hat dies seinen Grund darin, dass eine Handlung, die in die Gegenwart des Redenden fällt, nicht als wirklich abgeschlossen erscheint, sondern in der Regel in ihrem Verlaufe, also durativ, angeschaut wird.“ Vgl. Gentry, „Function,“ 370.

183 Vgl. James, „Imperfects,“ 187 für den Ausdruck der Aspektualität in der Koine. Moser, „Tense and Aspect.“

184 Für das NT siehe GGNT 201d. Siehe CGCG 33.46 zum schon klassisch seltenen Gebrauch.

185 Horrocks, „Envoi,“ 630. Hervorhebung nicht im Original. 
largely independent of one another“ seien. ${ }^{186}$ Empirisch untermauern lässt sich dies beispielsweise durch eine Studie von Buth, welcher sämtliche mindestens 10 Mal im NT dokumentierten Verben untersuchte, wobei er indikativische Formen und Partizipien ausschloss, um „purely aspectual choice“ (d.h. ohne weitere pragmatische Faktoren) zu untersuchen. ${ }^{187}$ Von den 418 Verben $^{188}$ wiesen 329 (d. h.: 78,7\%) beide Stämme auf. 189

\subsubsection{Einschränkung der Aspektwahl durch den gemeinten Situationstyp}

Dies bedeutet jedoch nicht, dass der Autor im konkreten Einzelfall in seiner Wahl vollständig frei gewesen wäre. ${ }^{190}$ Der obige schlaglichtartige Durchgang durch die Aspekte zeigt vielmehr, dass unter der Voraussetzung der Kommunikation eines bestimmten Situationstyps die Wahl des Aspekts bei manchen Verbkonstellationen obligatorisch ist. ${ }^{191}$ Unter Umständen ist diese Wahlfreiheit sogar auf der Ebene der Grammatik eingeschränkt, d. h., die Wahl ist sogar „formally obligatory,“ da alternative Verbformen in ungrammatischen Sätzen resultieren würden. ${ }^{192}$ Unter Umständen besteht im Verbsystem auch eine Lücke, die sicht schlicht aus den bisher etablierten Realisierungen eines Verbs in einer Sprache ergibt, wobei die Füllung in Übereinstimmung mit der Grammatik der Sprache wäre. ${ }^{193}$ Entgegen der unter Exegeten ${ }^{194}$ teilweise verbreiteten Annahme ist die Wahl des Aspekts also keine rein „subjektive“ Sache. ${ }^{195}$

Die Situation ist hier ganz ähnlich wie im Englischen, wo es möglich ist, zu sagen „Bloggs was drowning, but the lifeguard rescued him.“ aber nicht *,Bloggs drowned, but the lifeguard rescued him." ${ }^{196}$ Exemplarisch sei etwa auf das Verb $\varphi \varepsilon u ́ y \omega$ verwiesen, welches oft in Grammatiken als telisches Beispielverb ange-

186 Horrocks, „Envoi,“ 631.

187 Buth, „Verbs,“ 187.

188 Buth, „Verbs,“ 186.

189 Buth, „Verbs,“ 188.

190 Damit ist nicht im Alltagsverständnis gemeint, der Sprecher hätte hier seinen „freien Willen“ nicht wirken lassen können. Vgl. Bache, Study, 109: „In many instances, the selection of a particular member of a grammatical category may at the same time be both optional and obligatory - optional in the sense that the native speaker usually has a good deal of freedom to choose what to say and how to say it, obligatory in the sense that in a particular context, and relative to a particular conceptual unit, there may be only one well-formed expression.“

191 Vgl. Thomson, „Aspect,“ 32-33.

192 Bache, Study, 118.

193 Vgl. Bache, Study, 132 zu diesen ,systematic gaps.“

194 Siehe Thomson, „Aspect,“ 41 mit Bezug auf Porter, Verbal Aspect.

195 Thomson, „Aspect,“ 63 und 67.

196 Vgl. Thomson, „Aspect, 63.“ 
führt werden. ${ }^{197}$ Es ist im TLG-Korpus gut belegt und weist sowohl durative als auch aoristische Formen auf (von den gut 33.700 Vorkommnissen machen ca. 18.500 vom Präsens- und 11.800 vom Aorist-Stamm Gebrauch). Die Wahl des Aspekts durch den Erzähler ist deswegen aber sicherlich nicht einfach als „frei“ $\mathrm{zu}$ bezeichnen. Ebenso falsch wäre es zu sagen, es bestehe eine grundsätzliche „Tendenz“ des Verbs für die Darstellung durch einen bestimmten Aspekt. ${ }^{198}$ Vielmehr spiegelt die Statistik lediglich wider, wie verschiedene Plots zahlenmäßig im untersuchten Korpus vertreten sind. ${ }^{199}$

Denn der Ind. Aor. von $\varphi \varepsilon u ́ y \omega$ steht in der Regel dann, wenn in der Erzählung von einer Flucht die Rede ist, welche zum Abschluss kommt. ${ }^{200}$ (Vgl. etwa Xenophon, Hell. 1.2.16 mit Ipf. von $\delta ı \omega ́ \kappa \omega$ für die nicht erfolgreich zum Abschluss ${ }^{201}$ kommende Verfolgung.) Analog kann das Verb im Ipf. auch dann erscheinen, wenn

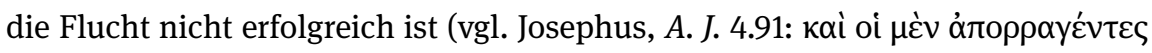

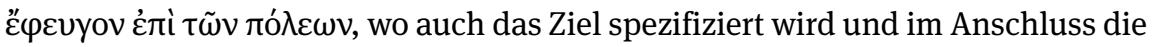
Vereitlung des Plans erzählt wird). ${ }^{202}$ Der Gebrauch des Ind. Aor. von $\varphi \varepsilon u ́ y \omega$ für

197 Z. B. GGNT $194 \mathrm{n}$.

198 GGNT 195j merkt an, dass es für „,solide Ergebnisse“ in der Textdeutung nötig sei, „mit Hilfe eines wissenschaftlichen Wörterbuches (besser noch mit Hilfe einer elektronischen Datenbank) [zu] klären, welche Aspektformen für das jeweilige Verb tatsächlich bezeugt sind bzw. welche Wahlmöglichkeiten der Sprecher/Schreiber gehabt haben dürfte." Dies ist eine sehr wichtige Feststellung (vgl. unten, Abschnitt 3.3.2.2, für den evtl. aoristischen Perfekt), darf jedoch nicht auf Mengenverteilungen der Belege für die verschiedenen Stämme ausgeweitet werden.

199 Wohl aus diesem Grund schließt Buth, „Verbs,“ 183 Indikativ-Formen aus seiner Analyse aus. Auch die Wahl des Aspekts von Infinitiven in Erzählungen dürfte aber vom Erzählten abhängig sein, wenn etwa in substantivierten Konstruktionen eine Referenz auf zuvor erzählte Situationen vorgenommen wird (vgl. 1. Kor 11,25: $\mu \varepsilon \tau \dot{\alpha}$ тò $\delta \varepsilon ı \pi v \tilde{\eta} \sigma \alpha \mathrm{l})$. Es ist daher sehr fraglich, inwiefern die Analyse zeigt, welchen Aspekt bestimmte Verben „bevorzugen.“

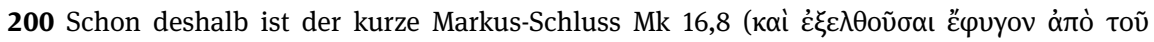

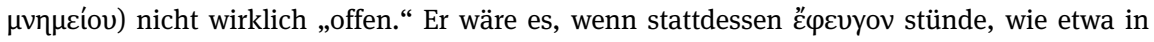

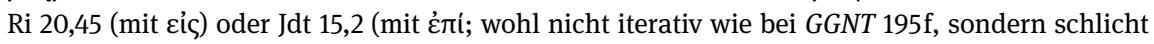

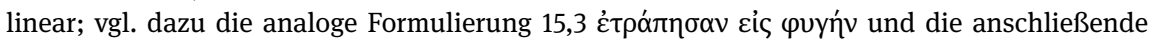

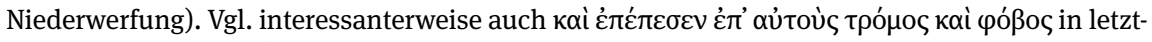

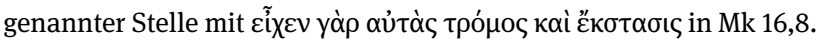

201 Was den telischen Status von $\delta เ \omega ́ \kappa \omega$ angeht, so gilt dieser wohl nicht für die Bedeutung, wie sie in Gal 1,13 vorliegt (BDAG 2059,2: ,to harass someone‘; vgl. demgegenüber die konativ zu interpretierende Ipf.-Form غ́тópӨouv im selben Vers), sondern nur in der Bedeutung (BDAG 2059,4): ,to follow in haste in order to find someth. 'Auf jeden Fall zu beachten ist: Natürlich kann auch Ipf. von $\delta \iota \omega ́ \kappa \omega$ für eine sich letztlich als erfolgreich herausstellende Flucht stehen, die an dieser Stelle des Erzählens aber noch als offen dargestellt werden soll (z. B. Homer, Ilias 22.158). 202 Vgl. Jdt 15,2-3. 
eine Flucht, die ihr Ziel nicht erreicht, erscheint demgegenüber problematisch wie „Bloggs drowned.“ in einer Erzählung, die mit Bloggs’ Rettung endet. ${ }^{203}$

Eine „Wahl“ zwischen Durativ und Aorist hat ein Erzähler also oft in dem Sinn, dass es meist Formen beider Stämme (seltener jedoch vom Resultativ) desselben Verbs gibt. Allerdings bringen sie dann nicht selten andere Situationstypen zum Ausdruck, sodass die Freiheit eben gerade nicht besteht, wenn man sie auf die Wahl einer sprachlichen Realisierung für ein bestimmtes Konzept bezogen versteht.

\subsubsection{Wahl der dargestellten Situation für die tatsächliche Situation}

Die Rede von der Wahlfreiheit ist hingegen wiederum legitim, wenn man damit lediglich sagen möchte, dass der Erzähler einen bestimmten Situationstyp bevorzugte, um ein tatsächliches Geschehen darzustellen. Denn die genannten, aspektuell bedingt variierenden Situationen können unter Umständen verschiedene Möglichkeiten sein, dieselbe tatsächliche Situation zu kommunizieren. ${ }^{204}$

203 Auffällig sind demgegenüber etwa Ri 8,12 und 2. Kön 9,27, wo auch $\delta เ \omega \dot{\kappa} \omega \omega$ aoristisch für die Verfolgung vorkommt. In gewisser Sicht ist die Flucht aber auch hier „erfolgreich,“ insofern ein Ziel erreicht wird, auch wenn dies keine Sicherheit gewährleistet. Sehr aufschlussreich ist in dieser Hinsicht Josephus, A. J. 12.272, wo zunächst von einem Fliehen „in die Wüste“ im Ind. Aor. die Rede ist (

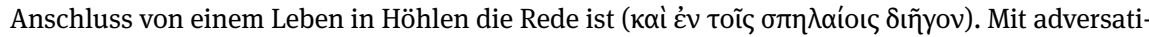
vem $\delta \varepsilon \dot{~ w i r d ~ d a n n ~ j e d o c h ~ f o r t g e f a h r e n ~ u n d ~ i m ~ I n d . ~ A o r . ~ e i n e ~ V e r f o l g u n g ~ e r z a ̈ h l t, ~ w i e d e r ~ „ i n ~ d i e ~}$

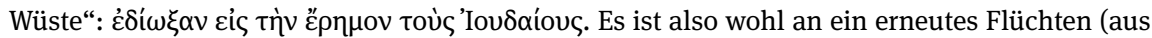
den Höhlen) gedacht. Dass das aoristische Verfolgen erfolgreich ist, zeigt 273. Nimmt man an, dass $\varphi \varepsilon v ́ y \omega$ das Verlassen eines Ortes A und das Erreichen eines Ortes B beinhaltet, so besteht immer noch eine gewisse Bandbreite in der Verwendung, da die Definition von B, dem Zielpunkt, relativ flexibel ist. Alternativ ist aber auch mit der Möglichkeit zu rechnen, dass $\varphi \varepsilon v ́ y \omega$ mit atelischer Semantik zu denken ist (den Hinweis verdanke ich Mike Aubrey), das Verb also gar nicht ein Erreichen von B impliziert und dieser Eindruck sich lediglich aus der Tatsache ergibt, dass in Erzählungen oft „die Flucht“ - dann wohl im Sinne eines bedrohten Unterwegsseins - im Aorist als Ganzes erscheint und die Referenz auch ein erreichtes Ziel beinhaltet. In diese Richtung könnte Josephus, A. J. 13.178 weisen: Zwar sagt hier 13.179 explizit, dass die Verfolgung nicht zum Ziel kommt (ov̉ $\mu \varepsilon ́ v \tau o l ~ \varphi \theta \alpha ́ v \varepsilon ı ~ \kappa \alpha \tau \alpha \lambda \alpha \beta \varepsilon \tilde{v}$ ), in 13.178 wird das Flüchten jedoch im Resultativ

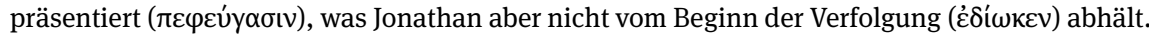
Umgekehrt ließe sich aber in Bezug auf dieselbe Stelle eventuell auch ein umgekehrtes Argument formulieren: Die Präsentation von atelischen Aktivitäten im Resultativ scheint selbst äußerst problematisch (колเó $\omega$ in Gal 4,11 ist in dieser Hinsicht sehr auffällig). Eventuell wäre hier also das Verlassen von A selbst als der auf ein Ziel (die äußere Grenze von A) zulaufendes Geschehen gedacht. Inwiefern der Resultativ einen solchen Situationstyp erzwingt oder dieser Gebrauch andersherum eine grundsätzlich telische Verbsemantik impliziert, muss hier aber offen bleiben. 204 Vgl. oben, Abschnitt 1.5.5 zu „The ship was in motion“ und „The ship moved.“ 


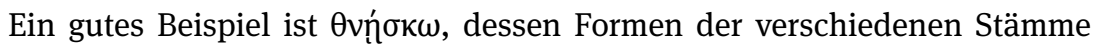
einem antiken Erzähler sehr durchgängig zur Verfügung standen. ${ }^{205}$ Für eine (zumindest innerhalb der erzählten Welt) tatsächliche Situation des Sterbens können Indikativ-Formen aller drei Stämme verwendet werden. Mit ع̋ $\theta \alpha v \varepsilon v$ kann auf den Moment des Sterbens Bezug genommen werden, wobei diskutiert werden kann, ob auch ein Verständnis als ,,accomplishment“ möglich ist, sodass der Ind. Aor. den zum Tod führenden Prozess inklusive des Umschlagens vom Leben in

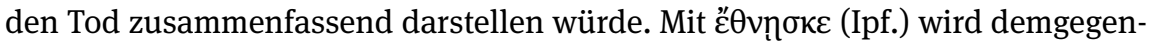
über ein Verhältnis der Betrachtzeit zu dieser Situationszeit gewählt, welches diesen Moment nicht beinhaltet - das Subjekt kann in einem weiteren Erzählschritt also überleben, muss es aber nicht. Überhaupt nicht mit dieser Betracht-

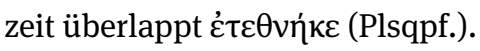

Es werden also verschiede Ereignisse erzählt, sowohl konzeptuell als auch was die Referenz angeht, liegen hier deutliche Unterschiede vor. ${ }^{206}$ Trotzdem können sie jeweils gewählt werden, um innerhalb des Erzählten dasselbe Element des Plots („der Tod des Helden“ etc.) zum Ausdruck bringen. Dabei ist dann allerdings erzähltheoretisch durchaus auffällig, wenn etwa nur der Prozess des Sterbens im Ipf. erzählt wird und der eigentliche Todeszeitpunkt übergangen wird. ${ }^{207}$

\subsubsection{Der Indikativ des Aorists als „unmarkierte“ Standard-Option der Narration}

In diesem sehr spezifischen Sinn ${ }^{208}$ ist es sicherlich mit Hinblick auf die meisten Verbkonstellationen $^{209}$ zutreffend, dass der Ind. Aor. in einer Erzählung die unmarkierte Option darstellt, also die natürlichste Wahl ist, um eines von vielen Ereignissen als Teil einer Erzählung zu kommunizieren:210

Durativ und Resultativ dagegen ... sind auch in ihrer Betrachtungs- bzw. Darstellungsweise spezifischer; sie sind auffällig bzw. „markiert“ und daher für die Textdeutung von besonderer Relevanz.

205 James, „Imperfects,“ 188 schreibt: „Speakers and writers of Greek had a choice about whether to present a process in the imperfect or an event in the aorist (both in contrast to a state in the perfect) in past time.“

206 Vgl. Bache, Study, 117.

207 Vgl. unten, Kapitel 8, Abschnitt 3 zur Ellipse.

208 Thomson, „Aspect,“ 73 warnt vor zu allgemeinen Aussagen darüber, „that a particular aspect always has the same markedness.“ Er verweist auf Comrie, Aspect, 121 für die Tendenz von der Unmarkiertheit des perfektiven Aspekts im Hinblick auf die Vergangenheit (und des imperfektiven Aspekts für die Gegenwart).

209 Dies gilt grundsätzlich nicht für Zustandsverben. Siehe unten, Abschnitt 3.4.2.3 zur perfektiven Darstellung von Verben ohne inhärenten Endpunkt.

210 GGNT 194. 
Die Signifikanz dieser ,aspektuellen Betonung “ kann natürlich auch genau darin bestehen, ein Geschehen als „lediglich“ im Vorlauf befindlich darzustellen, um es somit als Erzählhintergrund auszuweisen. ${ }^{211}$

\subsubsection{Subjektive Aspektwahl als Phänomen der Konzeptualisierung}

Auch wenn man die Kategorie des Aspekts daher gegenüber der Aktionsart als „subjektiv“ charakterisieren möchte, muss doch zumindest festgehalten haben, dass der grammatische Aspekt „,various ,objectively“ determined functions“ ausübt. ${ }^{212}$ Hiervon unterscheidet Bache die ,pure aspectual opposition, ${ }^{213}$ also eine tatsächlich subjektive Wahl zwischen „two ways of representing the situation,“214 die nicht bereits dadurch vorgegeben ist, dass der Sprecher einen bestimmten Situationstyp ausdrücken möchte und dies einen bestimmten Aspekt erfordert. $^{215}$

Im Folgenden soll „Aspektwahl“ in diesem Sinn folglich als ein Parameter der Konzeptualisierung verstanden werden, also als Entscheidung für eine von mehreren Möglichkeiten, Aussagen über Situationen zu „,konstruieren“ (vgl. „construal“). ${ }^{216}$ Es bleibt bei Wahlmöglichkeiten in diesem strikten Sinn der propositionale Gehalt des Satzes identisch, während dennoch semantische Unterschiede festgestellt werden können. ${ }^{217}$ Die Bedingungen, unter welchen sich die Proposition als wahr erweist, bleiben hier also trotz der Variation des Aspekts gleich. ${ }^{218}$

Diese rein aspektuelle Oppositon soll unten (Abschnitt 3.3 und 3.4) im Kontext der verschiedenen indikativischen Erzähltempora besprochen werden. Grundsätzlich beachtet werden muss, dass selbst die an und für sich mögliche

211 Vgl. unten Abschnitt 3.3.4 zur Reliefbildung.

212 Bache, „Aspect and Aktionsart,“ 67. Das Adverb steht in Anführungsstrichen, da die von einer Sprachgemeinschaft als akzeptabel angesehenen „verschiedenen Auffassungen ... [eines] Tatbestandes“ zu großen Teilen konventionell sind (vgl. Bache, „Aspect and Aktionsart,“ 68 mit Zitat von Jacobsohn, „Aspektfragen,“316).

213 Bache, „Aspect and Aktionsart,“ 68.

214 Bache, „Aspect and Aktionsart,“ 70-71.

215 Das volle Zitat bei Bache, „Aspect and Aktionsart,“ 70-71 (Hervorhebung ursprünglich in Kapitälchen) lautet: „Sometimes the speaker/writer has a ,subjective choice“ between two ways of representing the situation (in cases of pure aspectual opposition), sometimes he must choose one or the other way of representation (in cases where the aspects function in different ways in relation to tense and Aktionsart).“

216 Der Terminus „construal“ wird oft auch in der deutschsprachigen kognitiv-linguistischen Forschung beibehalten. Vgl. Blomberg und Jessen, „Einführung,“ 33-41. Siehe online z. B. Suñer Muñoz und Roche, „Konzeptualisierung.“

217 Bache, Study, 127.

218 Bache, Study, 126-130. Vgl. S. 130-131 zur nur eingeschränkt gegebenen Koreferrentialität. 
freie Aspektwahl im konkreten kommunikativen Kontext oft noch weiter eingeschränkt ist: „there may sometimes be little or no real choice between the aspects because of contextual or emotional considerations.“219 Auch soll hier darauf hingewiesen sein, dass die genaue Definition der griechischen Aspekt (nicht nur im Resultativ) noch immer Gegenstand teils heftiger Diskussion sind und sich in Abhängigkeit von der gewählten Lösung durchaus Unterschiede für den Umfang der Fälle ergeben, in denen tatsächlich freie Wahl zwischen den Aspekten zur Kommunikation derselben Situation besteht. ${ }^{220}$

\section{Tempus}

\subsection{Temporalität und griechische Tempora}

Das inhaltliche Gegenstück der grammatischen Tempora bildet die Kategorie der ,Temporalität. ${ }^{221}$ Temporalität - die gemeinte zeitliche Relation zwischen Äußerungsakt und dem zum Ausdruck gebrachten Sachverhalt - muss nicht notwendigerweise grammatisch (also durch die Tempora) ausgedrückt sein, sondern kann beispielsweise auch lexikalisch realisiert werden (z. B. durch Adjektive: „Mein gestriger/heutiger/morgiger Arbeitseinsatz ...").222

„Tempora“ im strikten Sinne 223 - d. h. als Untergruppen derjenigen grammatischen Kategorie, welche „die zeitliche Relation zwischen der Äußerungszeit und dem gemeinten Sachverhalt kennzeichnet“ - sind im Griechischen ausschließlich die Indikativ-Formen ${ }^{224}$ der drei Aspekt-Stämme Durativ, Aorist und Resultativ, 225 also Indikativ Präsens und Imperfekt, Indikativ Aorist, Indikativ

219 Bache, „Aspect und Aktionsart,“ 69.

220 Es wird unten, Abschnitt 3.3.3.2 im Zusammenhang mit der Wahl zwischen komplexivem Ind. Aor. und linearem Ipf. im Hinblick auf Tätigkeitsverben auf diese Diskussion kurz eingegangen.

221 GGNT 192b.

222 Siehe GGNT 192b, von wo auch das Beispiel übernommen ist.

223 Mit der Rede von „Tempora“ ist oft in einem weiteren Sinne die Kombination aus Tempus und Aspekt gemeint. Da diese Ausdrucksweise missverständlich ist, wird hier auf diese erweiterte Verwendung des Terminus verzichtet. Vgl. auch GGNT 192a.

224 Die Bezeichnungen „Indikativ Imperfekt“ und „Indikativ Plusquamperfekt“ sind tautologisch und werden daher in dieser Arbeit vermieden. So auch CGCG S. xli.

225 Entsprechend ist die Rede von „Tempusstämmen“ außerhalb des Indikativs eigentlich auch unpräzise (siehe GGNT 193a). Auch die Bezeichnung des Durativ-Stamms als „PräsensStamm“ und des Resultativ-Stamms als „Perfekt-Stamm“ ist so gesehen irreführend (vgl. GGNT 192g). Die Bezeichnung des Aorist-Stamms als „punktueller“ Stamm ist aus anderen Gründen 
Perfekt und Plusquamperfekt, sowie alle Formen des Futur-Stamms (der ein tatsächlicher und reiner ${ }^{226}$ Tempus-Stamm ist). ${ }^{227}$

In dieser Arbeit wird davon ausgegangen, dass die Indikativ-Formen des Altgriechischen - anders als die infiniten Formen - tatsächliche (absolute) Zeitbedeutung zum Ausdruck bringen. Das Augment hat die Funktion eines Vergangenheits-Zeichens, das aufgrund redundanter morphologischer Markierung $^{228}$ des Zeitbezuges allerdings auch fehlen kann. ${ }^{229}$

Dieses Verständnis widerspricht der im angelsächsischen Bereich ${ }^{230}$ vor allem von den Neutestamentlern Porter und Campbell vertretenen Position, wonach im Altgriechischen auch im Indikativ lediglich Aspektbedeutung vorliegt und der absolute Zeitbezug (ähnlich dem relativen Zeitbezug) kontextuell zu erschließen ist. ${ }^{231}$ Diese These beruht jedoch auf linguistisch problematischen Vorannahmen

problematisch, nämlich weil dadurch ein falsches Verständnis der durch den Aspekt ausgedrückten Perspektive nahegelegt wird.

226 D. h.: Der Futurstamm hat keine Aspektbedeutung. Dennoch kann im Hinblick auf Zukünftiges natürlich unterschiedliche Aspektualität vorliegen (siehe oben, Abschnitt 2.1). Im Neugriechischen ist diese - anders als in der Koine - auch grammatisch ausdrückbar.

227 Vgl. GGNT 192g. Als Sprache mit Aspektprominenz ist dabei natürlich zu beachten, ,that the nonindicative verbs have not ,lost" their tense markedness, but rather that only in the indicative mood do the verbforms gain tense“ (Ellis, „Aspect-Prominence,“ 144).

228 Vgl. Gentry, „Function,“ 369.

229 GGNT 64d. Gentry, „Function,“ 374 fasst die diachrone Entwicklung des Augments wie folgt gut zusammen: „The function of the augment in Hellenistic Greek must be understood from a diachronic perspective which includes a history of both aspect and tense in the verbal system. The system of the verb was originally aspectual that gradually became overlayered with tense as well. The addition of iota to morphemes marking only person and voice created the primary endings and present tense. This yielded a present/nonpresent arrangement. Then the creation of a future tense left the set of unmarked personal endings associated only with past tenses in the indicative. An adverbial prefix that originally had a deictic or spatial value developed into a marker with temporal semantic value. By 500 bc this was part of the morphology of the indicative past tenses, especially since apophonic reduction was no longer the sole means of marking the aorist. Within 150 years this double marking system deteriorated due to changes in pronunciation of vowels, accent and other phonological phenomena. By Byzantine and Modern times, only the syllabic augment was partially retained owing to education and stress." Vgl. auch Allan, „Tense“ im selben Band.

230 Siehe aber auch unten, Abschnitt 3.3.4.1 zu Weinrich, der meint (Tempus, 280): „Alle Tempora des Griechischen sind zeitlos.“

231 Porter, Idioms; Campbell, Indicative Mood, Non-Indicative Verbs und Basics. 
im Hinblick auf Kategorisierungen der Sprache ${ }^{232}$ und widerspricht dem Konsens unter Gräzisten. ${ }^{233}$

Anders als im Deutschen (und etwa auch dem Lateinischen) ist demgegenüber die relative Zeitbedeutung im Griechischen - d. h. das Verhältnis verschiedener Sachverhalte in der Vergangenheit untereinander/das verschiedentliche Verhältnis dieser Sachverhalte zur Äußerungszeit - grundsätzlich kontextuell zu erschließen und nicht Teil des durch die Verbformen Kommunizierten. ${ }^{234}$

Dies gilt natürlich auch für das Plusquamperfekt, welches naturgemäß oft auftritt, um Zustände zu bezeichnen, die bereits in der Vergangenheit realisiert waren (allerdings aufgrund des Aspekts nicht für andere Situationstypen der Vorvergangenheit). ${ }^{235}$ Nicht-indikativische Verbformen drücken zusätzlich auch kein absolutes Zeitverhältnis aus. Es lässt sich aber eine Korrelation zwischen verwendetem Stamm und zeitlichem Verhältnis zum übergeordneten finiten Verb feststellen (z. B. Ptz. im Durativ und Gleichzeitigkeit). ${ }^{236}$

\subsection{Deixis}

\subsubsection{Das Verhältnis von Betracht- und Orientierungszeit im Satz}

Der grundsätzlich deiktische Charakter der Tempora ist unbestritten. Das heißt, auch wenn hier von „absoluter“ Zeitbedeutung die Rede ist, ist dies immer in Rückbindung an die Äußerungszeit gemeint. ${ }^{237}$ Es ist weiterhin aber auch wichtig,

232 Vgl. GGNT 193a. Der prototypische Charakter der Vergangenheitstempora wird ausführlich behandelt von Fresch, „Typology,“ 404. Vgl. auch Allan, „Tense,“ 96-97 zur „deictic distance“ als vereinendes Merkmal des variierenden Gebrauchs der Tempora. S. 97: „In the prototypical case, distance is interpreted in terms of temporal distance.“ Auf einzelne Verwendungsweisen der Tempora, die nicht dem prototypischen Gebrauch entsprechen, wird im Laufe der Diskussion jeweils eingegangen. Es handelt sich dabei nicht um „Ausnahmen,“ sondern in der Regel (Fresch, „Typology,“ 404) um „typologically expected and pragmatically or lexically motivated deviations from the prototypical usage.“

233 Die Diskussion des von Porter, Idioms und Fanning, Verbal Aspect präsentierten Materials bei Caragounis, Development, 316-336 fällt wohl etwas zu engagiert aus. Auffällig ist jedoch in der Tat die fehlende Wahrnehmung in manchen exegetischen Arbeiten, dass es sich bei den Positionen von Porter und Campbell um Minderheitsmeinungen handelt.

234 GGNT 193b.

235 Siehe CGCG 33.40. Vgl. KG 385.

236 Generell hierzu GGNT 206c-i. Vgl. CGCG 33.57-66.

237 Vgl. hierzu Zifonun, „Textkonstitutive Funktionen,“ 318. 
$\mathrm{zu}$ beobachten, dass durch die Wahl der Tempora nicht einfach unbestimmte Ereigniszeiten für die Aussage festgelegt werden. ${ }^{238}$

Dies zeigt etwa der folgende Beispielsatz: „Hans arbeitete gestern nicht.“ Das Präteritum kann sich hier nicht schlicht auf den gesamten der Sprechzeit vorausgehenden Zeitraum beziehen. Es wird lediglich ausgesagt, dass für den durch „gestern“ markierten zeitlichen Rahmen kein Intervall gefunden werden kann, für welchen das Arbeiten des Hans' wahrheitsgetreu ausgesagt werden kann. ${ }^{239}$ Entsprechend wird eine tatsächliche Unbeschränktheit der durch das Tempus gebotenen Betrachtzeit im Text oft auch explizit spezifiziert (vgl. etwa 1. Kor 8,13:

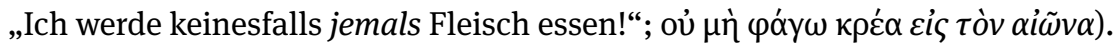

Zugleich wäre es aber auch zu kurz gegriffen, wenn man nun annähme, dass das Tempus schlicht auf die durch den linguistischen oder extra-linguistischen Kontext bereitgestellte Betrachtzeit Bezug nehme. Denn in Sätzen wie „Hans arbeitete heute nicht.“ liefert „heute“ eine Betrachtzeit, die über den Zeitpunkt der Äußerung hinausreicht - sodass keine Deckungsgleichheit mit dem auf Vorzeitigkeit beschränkten Zeitintervall des Tempus vorliegen kann. Zifonun schlussfolgert daher, „daß temporaladverbiale Betrachtzeit und tempusrelevante Betrachtzeit nicht in einem Verhältnis der Identität, sondern der Überlappung stehen."240 Tempora liefern also Betrachtzeiten in Abhängigkeit zu Sprechzeiten. Diese müssen mit den eventuell satzintern durch Temporaladverbialia bereitgestellten Betrachtzeiten überlappen - welche wiederum „mit der Ereigniszeit der Proposition überlappen.“241

Beispielsweise wird in Gal 1,19 durch den Vorvers eine Betrachtzeit festgelegt, die sich über 15 Tage erstreckt und auf ein bestimmtes Jahr datiert ist. Dieser Zeitraum ist zum Zeitpunkt des Briefschreibens bereits abgeschlossen, da sich an besagtes Jahr noch weitere Zeitintervalle anschließen (vgl. 1,18 und 2,1). Die durch den Ind. Aor. bereitgestellte Betrachtzeit umfasst somit diese im Kontext spezifizierte Betrachtzeit. Es gibt nun keine Ereigniszeit für den propositionalen Gehalt

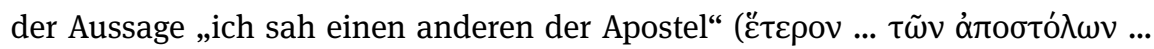

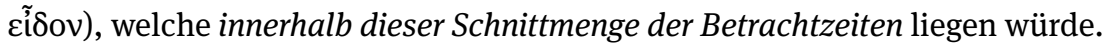

238 Das folgende Beispiel ist Zifonun, „Textkonstitutive Funktionen,“ 318 entnommen und die Erläuterung der Signifikanz lehnt sich eng an ihre Darlegung auf S. 318-319 an.

239 Ohne adverbiale Modifikation ist beim verneinten Ind. Aor. in der Tat häufig gemeint, ,that an event did not take place at any time anterior to speech time, “ was das Fehlen des Augments in solchen Fällen bei Homer erklärt: „The deictic relation between the speaker's deictic center and the temporal location of the state of affairs is therefore rather diffuse“ (Allan, „Tense“ 89).

240 Zifonun, „Textkonstitutive Funktionen,“ 319.

241 Zifonun, „Textkonstitutive Funktionen,“ 319. 


\subsubsection{Das Verhältnis von Sprech- und Orientierungszeit}

Bei zusammengesetzten Zeiten wie dem deutschen oder neugriechischen Futurperfekt wird zusätzlich zur Sprechzeit ein Hilfszeitpunkt eingeführt: In Sätzen

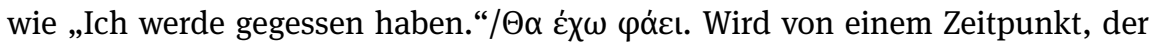
von der Sprechzeit aus in der Zukunft liegt, auf eine vollendete Situation zurückgeblickt. Diese „Orientierungszeit““242 kann im Text natürlich auch spezifiziert

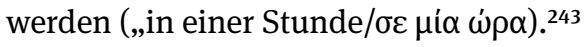

Man kann hier davon sprechen, dass zusätzlich zur Sprechzeit ein „,sekundäres deiktisches Zentrum“ im Text markiert ist. Hiervon zu unterscheiden ist der Fall, dass eine von der Sprechzeit abweichende Orientierungszeit diese als primäres deiktisches Zentrum ersetzt, es also zu einer Deixisverschiebung kommt. ${ }^{244}$ Dies soll im Folgenden gemeint sein, wenn von einer „Verschiebung der Orientierungszeit“ die Rede ist.

Der offensichtlichste Fall von Deixisverschiebung innerhalb eines Textes liegt vor, wenn an die Stelle der Sprechzeit des primären Sprechers (d.h. bei Erzählungen: des Erzählers der Rahmenerzählung) die Sprech(- oder Denk-)zeit einer anderen oder derselben Person (in einer Erzählung: einer Erzählfigur inklusive des erzählten Ichs) tritt. ${ }^{245} \mathrm{Im}$ Griechischen steht dabei auch in der indirekten Rede (im indikativischen Nebensatz) dasselbe Tempus wie in der entsprechenden direkten Rede, sodass lediglich Pronomina ${ }^{246}$ und die Anpassung der Person ${ }^{247}$ den Wechsel verrät. ${ }^{248}$

Auch das historische Präsens (siehe unten, Abschnitt 3.3.2.1) ist als Stilmittel $\mathrm{zu}$ verstehen, welches aus der Verschiebung der Orientierungszeit vom Standpunkt des Erzählers in die erzählte Zeit erklärt werden kann. Das Phänomen der „epistolarischen Tempora“ kann analog erklärt werden: Da im Medium des Briefes, anders als in der direkten mündlichen Kommunikation, Sprech-/Schreibzeit und Lesezeit auseinanderfallen, resultiert die Verschiebung des deiktischen

242 Zum in der Forschung teilweise verworrenen Verhältnis der Termini „Referenzzeit,“ „Evaluationszeit,“ „Betrachtzeit“ und „Orientierungszeit“ siehe Fabricius-Hansen, „Tempus,“ 737-738.

243 Siehe Duden 710.

244 Siehe Welke, Tempus, 17 zur Differenzierung.

245 Vgl. ähnlich Welke, Tempus, 17.

246 Vgl. aber 1. Kor 11,24, wo zusätzlich kein ötı steht.

247 Etwa Röm 9,11, 2. Kor 6,16; vgl. dazu ambivalentes Gal 5,2 und 1. Tim 4,1.

248 GGNT 206b. Vgl. GGNT 274cd. Entsprechend steht auch häufig ein ötı-Satz als Explikation

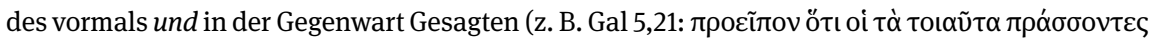
$\beta \alpha \sigma \iota \lambda \varepsilon i ́ \alpha v \theta \varepsilon o \tilde{~ o u ̉ ~ \kappa \lambda \eta р o v o \mu n ́ \sigma o v \sigma ı v) . ~}$ 
Zentrums auf die Lesezeit ${ }^{249}$ in einer Verwendung der Tempora, die nicht synchron mit dem auf die Schreibezeit bezogenen Gebrauch verläuft.

Am bekanntesten ist zweifelsohne der Fall, dass ein Vergangenheitstempus (meist Ind. Aor.) einen Sachverhalt zum Ausdruck bringt, dessen Situationszeit in der Zukunft der Äußerungszeit, aber eben im Vorfeld der Lesezeit, liegt (siehe unten, Abschnitt 3.4.2.1). Ebenso möglich ist jedoch der Gebrauch eines Gegenwartstempus für ebenfalls aus Sprecherperspektive Zukünftiges, wenn die Betrachtzeit mit der Lesezeit als neuer Orientierungszeit korreliert. Vor einigen Jahren hat Evans den in diesem Kontext relevanten „epistolarischen Perfekt“ einer vernichtenden Kritik unterzogen, auf die hier kurz eingegangen werden muss, da sie bisher keinen Widerspruch gefunden zu haben scheint.

Evans meint, es handle sich bei dieser Kategorie um eine „ghost function,“ die er in Analogie zu „ghost words“ versteht, welche in Wörterbüchern erschienen und immer wieder weitergegeben würden, obwohl sie im echten Sprachgebrauch nicht aufträten: ,[t] he original entry is usually the result of a mistake of some kind, but once accepted it continues to appear in lexica until someone has the patience to re-examine the evidence." ${ }^{250}$

Ironischerweise ist es Evans, der hier (trotz aller Geduld in der Auswertung der Evidenz) einen Fehler gemacht hat. Seiner Meinung nach erfordert die Rede vom „epistolarischen Perfekt,“ dass dieses im Sinn des Ind. Aor. als Vergangenheitstempus auftreten kann, was Evans jedoch für die Zeit des Neuen Testaments noch nicht als realisiert ansieht (siehe zu dieser Entwicklung unten, Abschnitt 3.3.2.2):251

The Ancient Greek perfect is a primary tense, while epistolary tense usage is a matter of historic tenses re-placing primary tenses, so as to suspend deictic simultaneity. The alleged epistolary perfect could only be a valid function if it contained some aoristic (and so historic) force.

Dabei übersieht Evans jedoch, dass es zu einem Auseinanderklaffen von Sprechund Orientierungszeit kommt, ${ }^{252}$ unabhängig davon, welche Betrachtzeit durch das Tempus (und adverbiale Bestimmungen) gewählt wird. ${ }^{253} \mathrm{Zu}$ keiner Anachronie kommt es bei entsprechenden Äußerungen von den beiden temporalen Stand-

249 Vgl. ähnlich Welke, Tempus, 17.

250 Zitat von Chadwick, Lexicographica, 13 bei Evans, „Ghost,“ 194.

251 Evans, „Ghost,“ 219.

252 Von Evans, „Ghost,“ 195-196 völlig richtig dargestellt.

253 Evans, „Ghost,“ 206: „Without the component of historic value we have a tense form which cannot replace a primary tense so as to suspend deictic simultaneity. Thus we do not have a tense capable of epistolary function.“ 
punkten aus nur im Hinblick auf auch zum Schreibzeitpunkt bereits Geschehenes und im Fall von auch zum Lesezeitpunkt noch Ausstehendes.

Anscheinend ist Evans dabei von der Darstellung von Mandilaras beeinflusst, der das epistolarischen Perfekt mit Aorist-Bedeutung in Verbindung bringt. ${ }^{254}$ Der ebenfalls von ihm angeführte Mayser gibt jedoch klar zu Protokoll, dass das „Perfektum im Briefstil präsentisch [steht],“ also mit Bezug auf „gegenwärtig[e] Handlungen, die der Briefschreiber erst vom Standpunkt des Adressaten als vollendet darstellt. “255 Dies scheint ganz in der Linie mit etwa dem, was Heinrich von Siebenthal zur Verwendung schreibt, dass sich das Perfekt nämlich ,auf einen (aus der Sicht des Sprechers/Schreibers) zukünftigen Zustand“ beziehe. ${ }^{256}$ Vielleicht sitzt Evans hier einem Missverständnis auf, da Mayser die Formulierung in

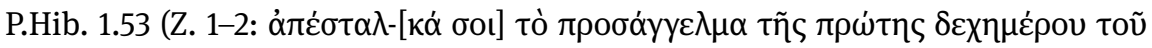
AӨù $\rho)$ mit ,,ich schicke dir hiermit“ übersetzt - was natürlich auch eine angemessene Auflösung des Ind. Aor. wäre.

In seinem Bemühen, die entsprechenden Verbformen als nicht-aoristisch zu erweisen und sie vor dem Hintergrund der „regulären“ Aspekt- und Tempusbedeutung $\mathrm{zu}$ verstehen, ${ }^{257}$ gelangt Evans $\mathrm{zu}$ einigen nicht haltbaren Interpretationen. Dies zeigt überdeutlich die Diskussion des Beispiels P.Petr. 2.2.2 an anderer Stelle. ${ }^{258}$ Der Brief datiert auf den 5. Pharmouthi (bzw. 21. Mai) $222 \mathrm{v}$.

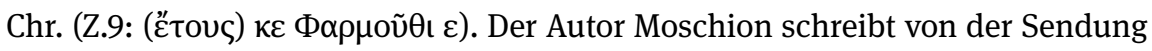
eines gewissen Dionysios zum Empfänger des Briefes, dem Strategen Diophanes, zur Schlichtung eines Streites. In Z. 5-6 erscheint dieses Ereignis mit folgen-

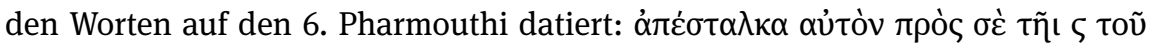
Фр$\mu$ ог̃ $\theta$. Evans meint daher, die Sendung finde erst am Tag nach Abfassung

254 Evans, „Ghost,“ 198 zitiert Mandilaras, Verb, § 475. Ich verstehe nicht, was mit „expressing the sense of the aoristic perfect combined with the meaning of the resultative perfect" gemeint sein soll. Kryptisch bleibt auch der wie folgt beschriebene pragmatische Effekt des epistolaren Perfekts gegenüber dem epistolaren Aorist: „it removes not only the time of writing to the time of reading, but also the writer himself to the side of the reader." Der Verweis auf abhängige futurische Konstruktionen (§ 477) legt auf jeden Fall nahe, dass Mandilaras dasselbe Phänomen in Blick hat wie Mayser.

255 Mayser, Grammatik, 2.1.183. Fanning, Verbal Aspect, 304 wird ebenfalls von Evans, „Ghost,“ 196 explizit zitiert. Obwohl Fanning nur in einer Fußnote auf den epistolaren Perfekt eingeht, ist doch deutlich, worin genau die Gemeinsamkeit mit dem epistolaren Aorist besteht: „This [use] has the same origin as the epistolary aorist discussed earlier, since it takes the viewpoint of the reader and locates the sense of the perfect in that time rather than in the time of the writer.“

256 GGNT 200h. Hervorhebung ursprünglich gesperrt.

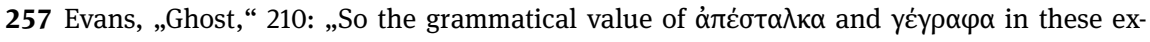
amples appears to be quite regular.“

258 Evans, Verbal Syntax, 164. 
des Briefes statt und das Verb könne daher keinen Vergangenheitsbezug haben. Offensichtlich ist das deiktische Zentrum hier aber einfach die Lesezeit: Zum Zeitpunkt, da Diophanes den Brief liest, gilt, dass die Sendung bereits erfolgte und der Betroffene unterwegs (beziehungsweise auch schon eingetroffen) ${ }^{259}$ ist.

Der Ind. Perf. hat also in der Tat seine „reguläre“ Tempusbedeutung, insofern hier sicherlich kein in die Vergangenheit des Schreibers weisender aoristischer Gebrauch vorliegt (ansonsten hätte Dionysos ja vor Abfassung ausgesandt werden müssen). Auch gibt es keinen Grund zur Annahme, der Ind. Perf. stehe

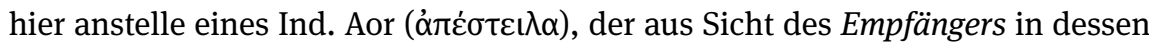
Vergangenheit zurückblicken würde. Dennoch liegt das Geschehen eindeutig in der Zukunft des Schreibers - und in der grammatisch völlig korrekt ausgedrückten Gegenwart des Lesers. Evans hingegen verharrt in der irrigen Annahme: „The focus of the perfect indicative is on Moschion's present state. " ${ }^{260}$ Er übersetzt entsprechend mit „I am sending him to you“261 - was aber eindeutig den durativen

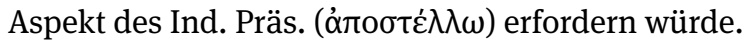

Fanning meint, der epistolare Perfekt sei im NT nicht belegt. ${ }^{262}$ Allerdings ist in Apg 15,27 innerhalb eines Briefes von der Sendung von Judas und Silas die

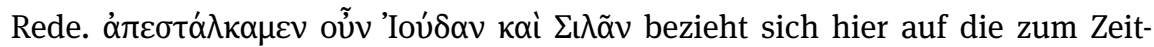
punkt des Schreibens noch ausstehende Sendung (vgl. für den Entschluss V. 22), welche dann in V. 30 partizipial aufgegriffen wird, ebenso wie das Lesen in V.

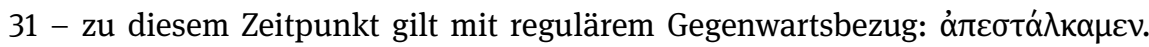

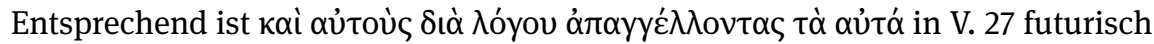
als „auch sie werden euch mündlich dasselbe sagen“ zu überstzen. ${ }^{263}$ Die Imple-

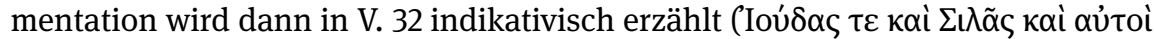

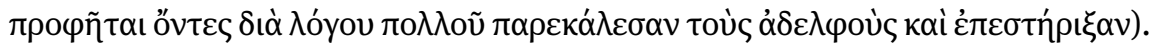

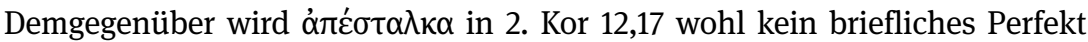
sein. Im Hintergrund steht die Frage, ob Paulus die Korinther indirekt - durch

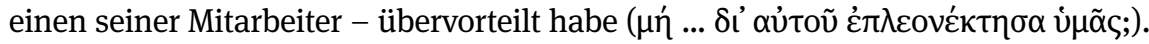
Die Frage ist, auf wen sich Paulus bezieht, wenn er dieses Verhalten als von „einem

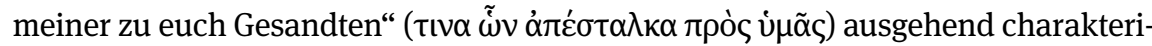

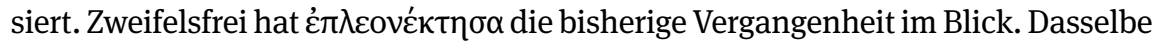

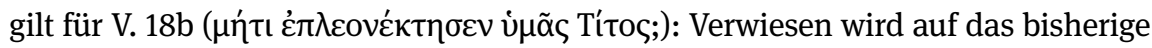

259 Auf der Außenseite ist der Brief auf den Folgetag datiert. Wie Mayser, Grammatik, 2.1.183 unmissverständlich festhält, „fällt die Absendung des Mannes mit der des Briefes zusammen.“ Für die Temporalangabe vgl. unten, Abschnitt $3.5 \mathrm{zu}$ 1. Kor 15,4.

260 Evans, Verbal Syntax, 164.

261 Vgl. Evans, „Ghost,“ 204 für eine ähnliche Paraphrase.

262 Fanning, Verbal Aspect, 304.

263 Zum Partizip siehe Robertson, Grammar, 891. 
Verhalten des Titus im Umgang mit den Korinthern (vgl. auch den komplexiven Aor.

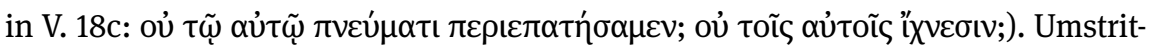

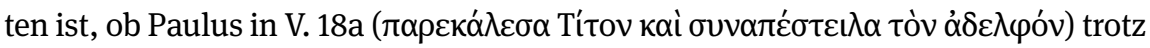
der Erwähnung nur eines Bruders nochmals auf die in 2. Kor 8,18.22 erwähnte (zum Zeitpunkt des Schreibens noch ausstehende) Sendung von Titus samt zweier Glaubensgeschwister verweist. Theoretisch wäre natürlich ein Verweis in V. 18a mit epistolarem Aorist und in V. 17 mit epistolarem Perfekt auf die noch ausstehende Sendung möglich. Gemeint wäre dann: „Hat euch denn einer derer, die ich jetzt zu euch schicken werde - bei einem früheren Besuch - schoneinmal übers Ohr gehauen?“ Doch werden die besagten Geschwister in Kapitel 8 als den Korinthern noch Unbekannte eingeführt. Gemeint ist durch den Ind. Aor. in V. 18a also wohl tatsächlich ein früherer Besuch (vgl. auf einer Linie damit 2. Kor 8,6). ${ }^{264}$ Der Ind. Perf. $\alpha \dot{\prime} \varepsilon \tau \sigma \alpha \kappa \alpha$ in V. 17 scheint demnach auf die Schreibzeit bezogen: Es geht um diejenigen, die von Paulus (bisher) gesandt worden sind und mit denen die Korinther dementsprechend bereits Erfahrungen haben sammeln können.

\subsubsection{Narrationstypen}

Ereignisse können grundsätzlich in einem vorzeitigen, gleichzeitigen und nachzeitigen Verhältnis zur Sprecher-Position stehen. Dem entsprechen nach Genette vier „Narrationstypen“:265

(1) Die klassische spätere Narration (in welcher ein Erzähler auf Ereignisse zurückblickt),

(2) die frühere Narration (etwa in der Prophetie),

(3) die simultane Narration und

(4) die sogenannte „eingeschobene“ Narration, bei welcher sich spätere und gleichzeitige Narration vermischen.

In graduelle Konzeptionen von Narrativität stellt das spätere Erzählen das prototypische Zentrum im Spektrum von Erzählungen dar, weil in diesem Fall im Sprechakt das tatsächliche Eintreten von Ereignissen am uneingeschränktesten zum Ausdruck kommen kann. ${ }^{266}$ Für die Analyse der Grammatik-Seite der Textstruktur sind dann diejenigen Tempora von Relevanz, welche Vergangenheitsbezug ausdrücken können.

264 Das genaue Verständnis der Passage hängt natürlich von der Frage nach der literarischen Eigenständigkeit von 2. Kor 10-13 ab und davon, wie man ein solches Fragment gegenüber dem in 2. Kor 8 angekündigten Besuch datiert. Vgl. Schmeller, Brief II, 351-352 (vgl. auch S. 72-74), der die Evidenz für letztlich nicht ausschlaggebend hält.

265 Genette, Erzählung, 140.

266 Vgl. Prince, Narratology, 150. 
Das Erzählen ist jedoch nicht auf die spätere Narration beschränkt. ${ }^{267} \mathrm{Im}$ Rahmen der simultanen Narration kommen daher auch Gegenwartstempora in Betracht. Im Hinblick auf letzteren Aspekt ist zu beachten, dass sich die Gegenwart „um den Sprechzeitpunkt herum [lagert]“ und nur „vage begrenzt“ ist: „Sie umfasst aus Sicht des Sprechers sein Jetzt und Geschehen, die zu dem Zeitpunkt schon begonnen haben oder ablaufen.“268 „Simultane Narration“269 muss in diesem Verständnis also nicht bedeuten, dass - wie etwa im Live-Kommentar zu einem Fußballspiel - eine (weitestgehend) durchgehende Deckungsgleichheit von Erzählzeit und erzählter Zeit der punktuellen Geschehnisse vorliegen muss. ${ }^{270}$ Selbst in diesem strikteren Verständnis von Simultaneität liegt jedoch ein gewisser temporaler Versatz zwischen Geschehen, Wahrnehmung und verbaler Bezugnahme - es sei denn, der Kommentator nimmt das noch ausstehende Ende einer Situation sprachlich bereits vorweg (und muss dann unter Umständen korrigieren). In diesem Fall geht die simultane Narration in die frühere oder „prädiktive“ Narration über.

Das Resultat einer solchen prädiktiven Narration wird hier gemäß der zugrundegelegten Definition nicht als „Erzählung“ bezeichnet. $\mathrm{Zu}$ beachten ist, dass in der englischsprachigen Exegese die Unterteilung des Diskurs-Typs „narrative“ in „story“ auf der einen Seite und „prophecy“ auf der anderen öfter begegnet (siehe Abb. 4). ${ }^{271}$ Verweise auf die „Zukunft in der Vergangenheit“ sind letztlich als Elemente der späteren Narration zu verstehen. ${ }^{272}$

Der letzte Typ, die eingeschobene Narration, ist der „komplexeste, da es sich um eine Narration mit mehreren Instanzen handelt und da sich die Geschichte und die Narration hier dergestalt verwickeln können, dass letztere auf erstere

267 Vgl. Weinrich, Tempus, 15 der sich gegen eine „Gleichsetzung von erzählten und von vergangenen Gegenständen“ ausspricht und im Rahmen seiner Tempustheorie ganz vielfältige „Anderszeite[en]“ berücksichtigen möchte.

268 Duden 708.

269 Für die „spätere“ Narration ist auch die Bezeichnung als „,nachzeitiges“ Erzählen gebräuchlich, statt des Adjektivs „simultan“ verwendet man oft „gleichzeitig.“ Diese Terminologie wird hier vermieden, da aus textgrammatischer Perspektive mit diesen Bezeichnungen anderes gemeint ist: So kann ein Geschehen als „gleichzeitig“ zu einem anderen Geschehen erzählt werden, aus der Perspektive des Erzählers jedoch in der Vergangenheit liegen: „Als er kam, ging ich fort.“ Ebenso können nachzeitige Verhältnisse im Modus der prädiktiven Narration zum Ausdruck kommen: „Ich werde ins Bett gehen. Zuvor werde ich die Zähne putzen.“ Siehe zu dieser Terminologie unten, Abschnitt 2.3.2.

270 Siehe unten, Abschnitt 3.4.2.2 zum „perfektiven/aoristischen Präsens.“

271 Für eine Übersicht der Einteilung von Longacre und Hwang, Holistic Discourse Analysis siehe oben, Kapitel 3, Abschnitt 3.4.4.2.

272 Es wird auf das Phänomen unten, Kapitel 11, Abschnitt 6 eingegangen. 
reagiert." 273 Dies gilt umso mehr, wenn es sich bei dem Erzähler zugleich um eine Figur des Erzählten handelt. So ist es möglich, dass ein Tagebuchschreiber einerseits die Ereignisse des Tages als vergangen notiert, andererseits aber die emotionalen Folgen der Geschehnisse bis in die Gegenwart der Niederschrift reichen. ${ }^{274}$ Es kann so zu einem nicht immer einfach nachzuvollziehenden Wechsel und unterschiedlich starkem Überlappen zwischen der Fokalisierung (siehe oben, Kapitel 3, Abschnitt 4.3) auf das erzählte und das erzählende Ich kommen.

Im Folgenden sollen die verschiedenen Tempora besprochen werden, die einem Erzähler für die jeweiligen Narrationstypen in der Koine zur Verfügung standen. Dabei ist die Klassifizierung immer auf den Zeitpunkt der Äußerung der jeweiligen Erzählebene bezogen. Die Verschiebung des deiktischen Zentrums innerhalb des Textes kann zu einer Variation der Tempora führen, betrifft aber nicht die temporale Relation des Erzählers zum Erzählten.

Der Schwerpunkt der Analyse wird auf der Frage liegen, wie die verschiedenen zur Verfügung stehenden Tempora eines Narrationstyps aufeinander bezogen sind. Dabei spielt die Tatsache, dass die „konkurrierenden“ Tempora (teilweise) aus verschiedenen Stämmen gebildet werden, eine wichtige Rolle.

Es wurde oben bereits darauf hingewiesen, dass es eine Funktion des Aspektes ist, den von einer Verbkonstellation ausgedrückte Situationstyp zu modifizieren. Abhängig davon, wie der Erzähler ein Ereignis der erzählten/zu erzählenden Welt betrachtet und dargestellt haben möchte, stehen ihm für diese Konzeptualisierungen in ihrer Aktionsart variierende Verben und die Reinterpretation der von ihnen evozierten Situationen durch den Aspekt zur Verfügung. ${ }^{275}$

Interpretativ „auffällig“ ist ein erzähltes Ereignis dann, wenn seine Darstellung (resultierend aus Aktionsart und Aspekt) nicht der gängigen Konzeptualisierung des Geschehens entspricht. Von einem grundsätzlich unauffälligen Erzähltempus der späteren Narration kann daher eigentlich keine Rede sein. Wenn beispielsweise für die gemeinten Charakteristika der Situation keine geeigneten Lexeme zur Verfügung stehen (z. B. Verbkonstellationen mit spezifischen Zustandsverben), dann ist hier die Wahl des „ungewöhnlicheren“ (d. h. seltenen) Resultativ-Stammes entsprechend auch nicht als auffällig anzusehen.

Von dieser Wahl der verschiedenen Typen an Situations-Schemata, die für die Darstellung eines tatsächlichen Geschehens (der erzählten und/oder realen Welt) herangezogen werden, und die unter anderem (d. h. zusätzlich zur lexikalischsemantisch basierten Aktionsart) durch die Aspektfunktion realisiert werden, ist

273 Genette, Erzählung, 140.

274 Das Beispiel stammt von Genette, Erzählung, 141.

275 Sowie natürlich weitere sprachliche Mittel, wie etwa die Verwendung bestimmter Adverbien. 
die rein subjektive aspektuelle Wahl zu unterscheiden, welche denselben Situationstyp unterschiedlich konzeptualisiert, also unterschiedliche Charakteristika in den Fokus rückt.

\subsection{Erzähltempora der späteren Narration}

\subsubsection{Hinführung}

An Vergangenheitstempora stehen der späteren Narration grundsätzlich die Tempora Indikativ Aorist, Imperfekt und Plusquamperfekt zur Verfügung. In den Paulusbriefen ist eine Plusquamperfekt-Form ausschließlich in Röm 7,7 belegt und vertritt dort das Ipf. von oĩ $\alpha$ (Ind. Perf. mit Ind. Präs.-Bedeutung) in einem irrealen Konditionalgefüge. ${ }^{276}$

Im Folgenden liegt daher der Schwerpunkt auf der Frage, in welchen Fällen ein Erzähler tatsächlich zwischen durativem und aoristischem Aspekt - beziehungsweise zwischen den Erzähltempora Ind. Aor. und Ipf. - im Hinblick auf die Verbkonstellation eine freie Wahl hat und welche Konsequenzen sich für die Erzählung ergeben.

Zunächst muss jedoch auch kurz auf die Möglichkeit eingegangen werden, dass Verben, die der Form nach Gegenwartstempora zuzuordnen sind, für Ereignisse herangezogen werden, die aus Sicht des Erzählers in der Vergangenheit liegen. Sprachliche Möglichkeiten, Vergangenes als aus früherer Perspektive zukünftig aber aus Sprecherperspektive geschehen darzustellen, werden hier ausgeklammert und unten (Kapitel 11, Abschnitt 6) kurz besprochen.

\subsubsection{Gegenwartstempora mit Vergangenheitsbezug}

\subsubsection{Historisches Präsens}

Das „historische/narrative“ (indikativische) Präsens „hebt typischerweise Neues und Entscheidendes hervor und steigert dadurch die Lebhaftigkeit der Darstellung."277 Dies wird erreicht, indem die Wahl des Gegenwartstempus für Vergangenes den Eindruck erweckt, ,as if an action that occurred in the past occurs in the present.“"278 Es findet also eine Verschiebung der Orientierungszeit statt, was in einer geringeren temporalen Distanz resultiert - und damit einhergehend, so

276 Vgl. NSS 921. Vgl. Gal 4,3 für die periphrastische Konjugation: „wir waren unter die Elemente

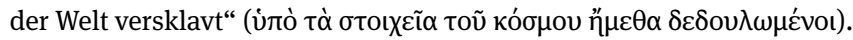

277 GGNT 197d.

278 CGCG 33.54. 
die allgemeine Ansicht, in einer „Lebhaftigkeit,“ die man narratologisch auch mit geringerer narrativer Distanz erfassen könnte. ${ }^{279}$

Diese Ansicht scheint zumindest im Hinblick auf telische Verbkonstellationen plausibel. ${ }^{280}$ Es liegt dann in der Regel keine aspektuelle Opposition vor, da der Erzähler hier im strikteren Sinn „simultan“ gleichzeitiges berichtet, sodass keine Zeit für eine lineare Binnenperspektive bleibt. ${ }^{281}$ Primär ist dabei aber der rhetorische Effekt „that is ... based on a mismatch of time.“282

Andere Forscher/-innen betrachten das historische Präsens primär als ein Mittel der Reliefgebung, ${ }^{283}$ was bei Verben des Sprechens damit begründet werden kann, dass die Sprechhandlung selbst oft nicht besonders hervorgehoben, sondern vor allem den Inhalt der Äußerung betont zu werden scheint. Teilweise wird dafür argumentiert, diese pragmatische Funktion generell auf das historische Präsens zu übertragen. ${ }^{284}$ Narratologisch könnte man das historische Präsens dann als Fokalisierungssignal verstehen, welches eine Wertung des Erzählers erkennen ließe:285

At least in Matthew, the [historical present] is an editorial device to indicate thematic prominence: an aid to the reader or listener to discern the hierarchy of themes present, and in particular to know which themes are of intrinsic interest to the author himself.

Es ist aber sehr fraglich, ob das klassisch mit „Lebhaftigkeit“ Gemeinte tatsächlich durch eine Übersetzung in die Kategorie der „prominence“ sinnvoll eingefangen wird. ${ }^{286}$ Im Hintergrund steht oft die Annahme, das historische Präsens sei

279 Vgl. auch oben, Kapitel 3, Abschnitt 4.3. Siehe jedoch auch für Details Abschnitt 3.3.3.2 in diesem Kapitel.

280 CGCG 33.54 beschränken den Gebrauch mehr oder weniger auf solche Verben: „It occurs nearly exclusively with telic verbs.“

281 Vgl. CGCG 33.20. Es handelt sich hierbei letztlich um einen (durch die Verschiebung der Orientierungszeit ergänzten) Sonderfall des „perfektiven/aoristischen“ Ind. Präs. Siehe unten, Abschnitt 3.3.2.2.

282 Buth, „Participles,“ 305, der feststellt, dass abhängige Partizipien den durativen Aspektwert durchgängig beibehalten. Dies sei nicht zu erwarten, wäre die Hauptfunktion des historischen Präsens, imperfektive Aspektualität einzuführen.

283 Siehe hierzu auch unten, Abschnitt 3.3.4.

284 Vgl. James, „Imperfects,“ 210-212.

285 Robar, „Historical Present,“ 351 . Hervorhebung nicht im Original.

286 Dafür argumentiert Robar, „Historical Present,“ 333: „If we rechristen vividness as prominence, that is, attracting additional processing time than the surrounding material, we can make some headway as Runge indicated. The ,vividness ' is not temporal transport, from the past to the present, but it does arrest the attention and force the reader to evaluate what is happening within the text, whether it be structural prominence or thematic prominence." Der Zusammenhang zwischen temporaler Distanz, narrativer Distanz (Anschaulichkeit) und Reliefgebung erscheint 
übersprachlich nicht mit einer Nivellierung von Aspekt und Tempus verbunden, sondern sei schlicht auf einer anderen Ebene angesiedelt. ${ }^{287}$ Der Duden gibt allerdings für das Deutsche den temporalen Transport weiterhin als Grundfunktion an: „Beim szenischen Präsens ... wird das vergangene Geschehen dadurch gleichsam in die Gegenwart - oder das Jetzt des Sprechers umgekehrt in die Vergangenheit - transportiert (Vergegenwärtigung, Verlebendigung)." ${ }^{288}$ Auch das historische Präsens im Griechisch lässt sich zufriedenstellend (und in Übereinstimmung mit etwa dem Gebrauch epistolarischer Tempora; siehe oben, Abschnitt 3.2.2) als Resultat der Deixisverschiebung verstehen. ${ }^{289}$ Die im Einzelnen bei den Lesern erzielte/zu erzielende Wirkung sollte demgegenüber wohl letztlich abhängig vom Genre/der Textsorte und unter Einbeziehung pragmatischer Gesichtspunkte vor dem Hintergrund der primären temporalen Verschiebung diskutiert werden.290

Auch innerhalb der neutestamentlichen Erzählwerke varriiert der Gebrauch des historischen Präsens stark (151 bei Mk, gut die Hälfte davon mit Verben des Sprechens; 9 Vorkommnisse bei Lk). ${ }^{291}$ In seiner Durchsicht sämtlicher indikativischer Präsens-Formen im NT identifiziert Battle Jr. keinen historischen Präsens in den Paulusbriefen. ${ }^{292}$

Diskutiert wird der Gebrauch immer wieder für Röm 7,14-24, was die Interpretation einer Erzählung aus der Perspektive des erlebenden Ichs der Vergangenheit anstelle einer simultanen Narration erlauben würde. Das Urteil Wallaces,

nicht so deutlich, wie er in der Literatur öfter dargestellt wird. Eine gründliche Untersuchung, welche die verschiedenen Ebenen zunächst sorgfältig auseinanderhält, um sie dann sinnvoll aufeinander beziehen zu können, wäre wünschenswert.

287 Robar, „Historical Present,“ 332.

288 Duden 724. Vgl. Breindl, „Temporalität, Aspektualität und Modalität“ 209-210: „Die vergangenheitsbezogene Verwendung kann dann als eine sprechzeitbezogene ,Verschiebung einer der Koordinaten beschrieben werden. Das kann nach dem allgemeineren Konzept der Deixisverschiebung (oder Origoverschiebung) modelliert werden, bei der das deiktische Zentrum - im Fall von Tempus $\mathrm{S}$ - auf ein sekundäres, von $\mathrm{S}$ zu unterscheidendes deiktisches Zentrum verschoben wird ... Eine quasi umgekehrte Lösung schlägt Ek[, Präsens] vor, die von einer ,Bildfunktion` ausgeht, bei der der Sprecher den Sachverhalt, über den er spricht, wie ein zu beschreibendes Bild als eine ,fiktive Gegenwart‘ sozusagen in das Sprechzeitintervall hereinholt.“

289 Vgl. Thomson, „Aspect,“ 27.

290 Vgl. bereits KG 382: „Auch diese Ausdrucksweise ist allen Sprachen gemein, und zwar nicht bloss als Form der lebendigen und anschaulichen Schilderung, sondern auch in dem nüchternen Stile der Chroniken und Genealogieen; denn auch der Chronist versetzt sich in das Jahr zurück, dessen Ereignisse er aufzählt.“

291 GGNT 197d.

292 Battle Jr., Present Indicative, 220 klassifiziert so кpíveı in Röm 2,1 als historisches Prä-

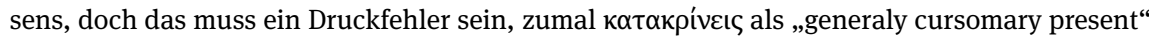
(vgl. S. 49) eingeordnet wird. 
da Paulus hier in der 1. Sg. Spreche, sei diese Einordnung „not at all likely,“293 überzeugt wenig. Seine Einschätzung, das historische Präsens könnte generell in der griechischen Literatur auf die 3. Sg./Pl. Beschränkt sein, ${ }^{294}$ ist auf jeden Fall unzutreffend. 295

Entscheidendes Gegenargument sind vielmehr die zum Ausdruck kommenden Situationstypen: Das Handeln und Wollen in V. 15 hat beispielsweise

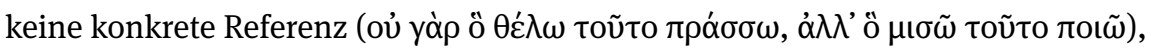
sondern hat Wiederholtes im Blick. Das telische Geschehen in Röm 7,21 hingegen könnte, in Isolation gelesen, durchaus als historisches Präsens gelesen werden

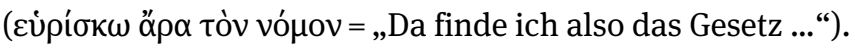

Bedenkenswert wäre wohl letztlich lediglich die an Petrus gerichtete Frage

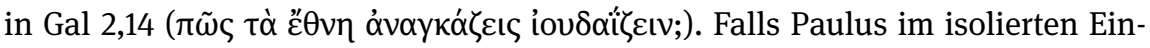

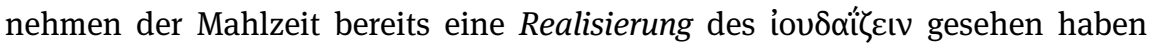
sollte, ist durchaus möglich, dass sein Vorwurf hier zu paraphrasieren wäre als: „Ich finde es unglaublich, dass Du die Heiden zum jüdischen Lebensstil gezwungen hast.“ Kühner/Gerth merken zumindest die Möglichkeit des Gebrauchs „im Dialog, wenn eine Person sich lebhaft in die von der anderen geschilderte Situation versetzt“" an. ${ }^{296}$ Das Präsens wäre dann natürlich gerade nicht konativ zu verstehen. ${ }^{297}$

\subsubsection{Aoristischer Indikativ Perfekt?}

Die finale Phase der Entwicklung des griechischen Ind. Perf. führt zu einer funktionellen Verschmelzung mit dem Ind. Aor., durch welchen das Tempus dann vollends verdrängt wird. ${ }^{298}$ Die Frage, wann der Ind. Perf. sich zum Vergangenheitstempus - ohne aspektuelle Unterscheidung von Ind. Aor. - entwickelt hat, ist nach wie vor sehr umstritten. ${ }^{299}$ (Die Entwicklung ist, entgegen der Behauptung von Evans, keine Voraussetzung für den „epistolarischen Perfekt“; siehe oben, Abschnitt 3.2.2.)

293 Wallace, Grammar, 531.

294 Wallace, Grammar, 528.

295 Vgl. CGCG 33.54 mit Verweis auf Lysias, Orat. 1.24-25. Siehe aber bereits auch die Beispiele bei KG 382.

296 KG 382.

297 Siehe unten, Abschnitt 3.4.1.1.

298 GGNT 200f.

299 Allan, „Tense,“ 112 verweist auf Duhoux (Abschluss im 2. Jh.) und Porter/McKay (4./5. Jh.), legt sich selbst jedoch nicht fest. Für eine Diskussion der vorgebrachten Stellen im NT siehe etwa Fanning, Verbal Aspect, 299-303. Er legt sich auf folgende Stellen für diese Kategorie fest: Mt 13,46, Apg 7,35, 2. Kor 2,13 und 11,25, Offb 5,7, 7,14, 8,5, $19,3$. 
Immer wieder wird argumentiert, dass auch bereits einige Perfekt-Formen im NT diesen Wandel belegen, Ind. Perf. also lediglich im Austausch zum Ind. Aor. steht. ${ }^{300}$ So wäre dann etwa in 2. Kor 12,8-9 schlicht erzählt, dass in der Vergangenheit Paulus drei Mal zum Herrn betete (Ind. Aor.; $\pi \alpha \rho \varepsilon \kappa \alpha ́ \lambda \varepsilon \sigma \alpha \alpha)$ und dieser ihm

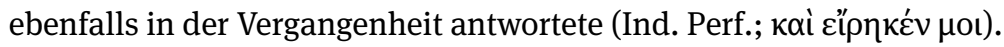

Allerdings geht die morphologische Angleichung der beiden Tempora ihrer funktionellen Vereinigung wohl deutlich voraus und ist Teil einer umfassenderen Vereinfachung der griechischen Verbformen. ${ }^{301}$ Es ist daher auch schlicht die Option einer unzureichenden Differenzierung der Form durch den Autor zu erwägen, ohne dass eine generelle Auflösung der Aspektunterscheidung (welche erstaunlich erscheinen würde, da „im NT [Ind. Aor. und Ind. Perf.] fast durchweg sorgfältig auseinandergehalten“302 werden) angenommen werden müsste: „Da es sich durchgängig um nicht eindeutig reduplizierte Formen handelt, wurden sie in der Volkssprache weiterhin nicht als Perfekte, sondern als Aoriste eingeordnet und verwendet.“303

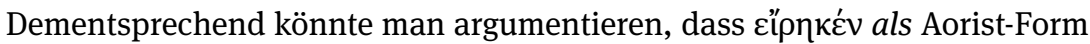
verwendet wurde. Entsprechend läge hier (wie freilich auch im Fall einer funktionellen Verschmelzung) keine aspektuelle Schwerpunktsetzung in der Konzeptualisierung des Geschehens vor. Allerdings ist neben der besagten Perfekt-Form in 2. Kor 12,9 in den Paulusbriefen der Ind. Aor. von $\lambda \varepsilon \dot{y} \omega$ immerhin 6 Mal belegt

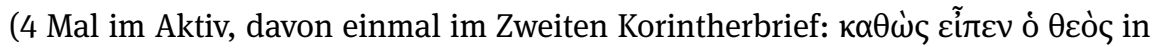

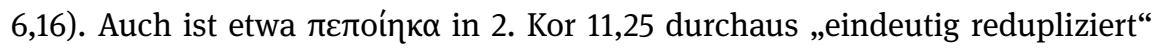
und erscheint im Kontext einer aoristisch formulierten späteren Narration.

\subsubsection{Historischer Indikativ Perfekt?}

Eine alternative Lösung ${ }^{304}$ stellt daher die These dar, der Ind. Perf. brächte in vielen der vorgebrachten Stellen einen „Zustand in der Vergangenheit“ zum Ausdruck, ${ }^{305}$ stünde also für das Plsqpf. Analog zum „historischen Präsens“ wird ein solcher Gebrauch auch als „historischer Perfekt“ bezeichnet.

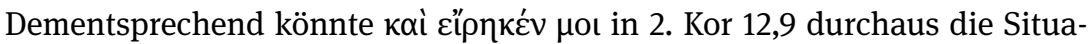
tion der Antwort des Herrn als „,vorliegender Entschluss“ (und nicht nur als Akt

300 Sehr ausführlich hat dafür Caragounis, New Testament, 136-168 argumentiert.

301 Evans, „Ghost,“ 202-206.

302 GGNT 200e.

303 GGNT $200 f$.

304 Evans, „Ghost,“ 205 erklärt so etwa einen Teil der für die aoristische Interpretation des Ind. Perf. vorgebrachten Evidenz. GGNT 200g nennt schlicht die Möglichkeit und bezieht diese nicht auf GGNT 200f.

305 GGNT 200g. 
der Mitteilung im Ind. Aor./Ipf.) konzeptualisieren, wobei die Situation aber eben in der Vergangenheit zu verorten wäre und in späterer Narration eine Sequenz (aus Bitte und vorliegender Antwort) dargestellt wäre.

Crellin (und Runge?) ${ }^{306}$ wenden sich mit folgendem Argument gegen die von Robertson als Belege des historischen Perfekts angeführten Gebrauch des Ind. Perf., etwa in Mt 13,46 (vgl. Abb. 23): „Eine besonders wertvolle Perle gefun-

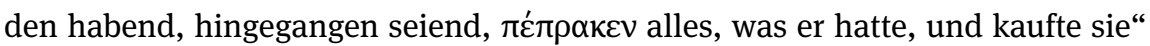

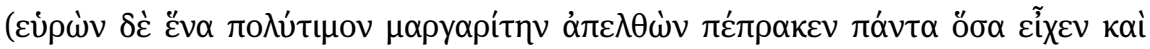

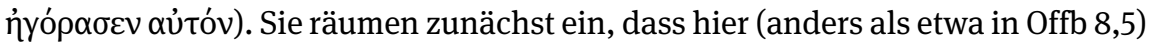
die Betonung (,prominence“) durch das eigentliche Gegenwartstempus durchaus nachvollziehbar wäre. Andererseits: ${ }^{307}$

[I]t is hard to see why in these examples one would use the perfect to do this, in preference to the historic present, since the latter would unambiguously place focus on the event of selling, as opposed the postevent situation, as would be the case if the perfect had its former semantic value.

Dieser Einwand scheint nur teilweise berechtigt. Es ließe sich nämlich durchaus anmerken, dass die Konzeptualisierung des Geschehens mit Fokus auf dem Zustand, in welchem bereits alles verkauft ist und der potenzielle Käufer somit das notwendige Kapital für den anstehenden Kauf besitzt, durchaus Sinn macht,

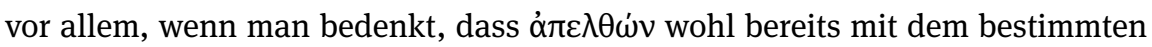
Ziel des Verkaufens zu denken ist, dessen Erreichen im Ind. Perf. = Plpqpf. dann bereits vorausgesetzt wäre. ${ }^{308}$

Beachtenswert ist die Tatsache, dass als Belege für das „historische“ Perfekt diskutierte Formen gerade in Schriften auftauchen, in welchen das Plusquamperfekt unterrepräsentiert ist. Es ist im NT insgesamt nur $86 \mathrm{Mal}$ in 35 verschiedenen Formen belegt, zieht man hiervon die Formen von oĩ $\delta \alpha$ und ǐ $\sigma \tau \mu \mathrm{t}$ ab

306 Crellin, „Semantics,“ 453 beschließt die Fußnote mit den Worten „My thanks to Steve Runge for these references." Es ist nicht klar, inwiefern Runge auch die Beurteilung beigesteuert hat.

307 Crellin, „Semantics,“ 453.

308 Die Wahl des Gegenwartstempus könnte dadurch erklärt werden, dass für das Verb keine Plsqpf.-Form existiert. Allerdings ist das gewählte Beispiel wohl ohnehin nicht sonderlich reprä-

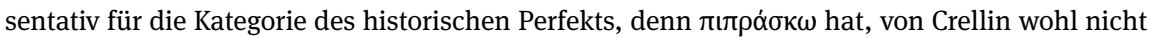
bemerkt, auch keinen (nennenswert belegten) aktivischen Ind. Aor., sodass die Wahlmöglichkeit des Evangelisten hier ohnehin sehr eingeschränkt ist (vgl. oben, Abschnitt 2.3.4) und argumentiert werden kann, dass in diesem speziellen Fall der Resultativ wohl anstelle des Aorists verwendet wird. NSS 87 nimmt daher einen Gebrauch anstelle des Ind. Aor. an. Allerdings kennt der Autor zumindest den Aorist-Stamm des augenscheinlich synonymen $\pi \omega \lambda \varepsilon \dot{\varepsilon} \omega$ (siehe Mt 19,21), sodass die Wahl dieser Verbform doch erklärungsbedürftig bleibt und nicht allein aus mangelnden Alternativen begründet werden kann. 
(die Ipf.-Bedeutung haben), so bleiben nur insgesamt 39 Vorkommnisse. Außerhalb der Erzählwerke ist ein solches Plsqpf. im NT aber sogar nur in 1. Joh 2,19 ( $\mu \varepsilon \mu \varepsilon v \eta ́ \kappa \varepsilon ı \sigma \alpha v)$, im Rahmen eines irrealen Konditionalgefüges, belegt. In den

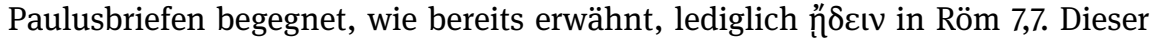
Verteilung steht die auffällige Häufung von als mögliche Belege herangezogenen Vorkommnissen des Ind. Perf. mit Plusquamperfekt-Bedeutung gerade im Zweiten Korintherbrief spannungsvoll gegenüber. Dasselbe gilt für die Johan-

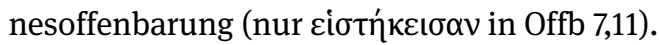

Diese Datenlage könnte dahingehend ausgewertet werden, dass die Verfasser dieser Schriften mit dem Plusquamperfekt nicht gut vertraut waren und daher auf den Ind. Perf. als Ersatz zurückgriffen. ${ }^{309}$ Allerdings ist gerade im JohannesEvangelium, welches ebenfalls in besonderem Maß „historische“ Perfekt-Verben aufweisen soll, ${ }^{310}$ auch ein ausführlicher Plusquamperfekt-Gebrauch belegt. ${ }^{311}$ Eventuell unterscheiden sich Johannesoffenbarung und Paulusbriefe daher vom vierten Evangelium dahingehend, dass die Autoren insgesamt selten auf vergangene Situationen Bezug nehmen, die sie (anders als im Ipf.) zusätzlich als Resultat konzeptualisiert haben wollen. Dann müsste freilich eine andere Erklärung dafür gefunden werden, weshalb der Ind. Perf. oft „untypisch“ gebraucht wird, zumal, wenn man den Gebrauch anstelle des Ind. Aor. ebenfalls für nicht plausibel hält.

Aufschlussreich ist in dieser Hinsicht, wie Robertson das „historische Perfekt“ für 2. Kor 2,13 (gegenüber der Alternative einer aoristischen Interpretation) begründet:312

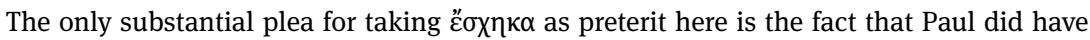

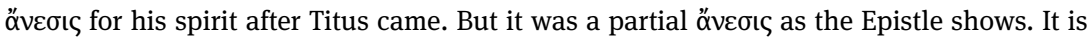
therefore possible that in 2 Cor. 2:13 we do have a present perfect = preterit punctiliar (cf. $\dot{\varepsilon} \xi \tilde{\eta} \lambda \theta$ ov), possible but not quite certain. Paul may have wished to accent the strain of his anxiety up to the time of the arrival of Titus. The aorist would not have done that. The imperfect would not have noted the end of his anxiety. It was durative plus punctiliar. Only the past perfect and the present perfect could do both. The experience may have seemed too vivid to Paul for the past perfect. Hence he uses the (historical dramatic) present perfect.

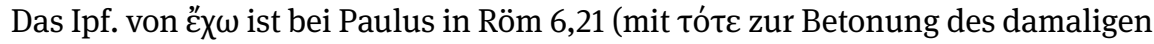
Habens) belegt. Plsqpf. ist selten, aber etwa in Res. Gest. 10 belegt ('A $\rho x \varepsilon \rho \omega \sigma u ́ v \eta v$,

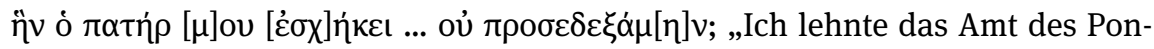

309 Siehe oben, Abschnitt 2.3.4 zum „systematic gap.“

310 Robertson, Grammar, 897: „This vivid perfect is found in John's Gospel in particular. One only needs to have some imagination himself.“

311 Joh 4,8, 6,17, 7,30, 8,30, 9,22, 11,13.19.30.44.57.

312 Robertson, Grammar, 900-901. 
tifex Maximus ab [Ind. Aor.], welches mein Vater innegehabt hatte [Plsqpf.]“). Paulus hätte also durchaus Wahlmöglichkeiten für die verschiedenen Konzeptualisierungen des Geschehens gehabt.

Bemerkenswert ist, dass Robertson die Kategorie des historischen Perfekts hier explizit an die Erfahrung des Autors zurückbindet, der als Erzählfigur seiner eigenen Erzählung auftritt. Der Gebrauch des Tempus scheint also durchaus mit einer starken Gegenwartskomponente begründet, der Tatsache, dass dem Erzähler das Erzählte noch „lebhaft“ vor Augen steht. Der Ind. Perf. ist hier also letztlich gerade nicht ,wie das Plsqpf“313 gebraucht, da dieses in Robertsons Sicht zu sehr ein Ende der in die Gegenwart auf gewisse Weise fortbestehenden Situation impliziert hätte.

Primär scheint also zumindest in dieser Darstellung das „historische Perfekt“ gar nicht eine alternative Konzeptualisierung vergangener Situationen darzustellen (sich anschließender Zustand vs. vorausgehendes Ereignis im Ind. Aor.), die gegenüber dem hierfür ebenfalls möglichen Plsqpf. mit einer stärkeren Betonung aufgrund der Verschiebung der Orientierungszeit in die Vergangenheit verbunden wäre.

Vielmehr scheint die Betrachtzeit so verschoben, dass sie die mit der Sprechzeit koinzidierende Orientierungszeit umfasst - was genau der standardmäßigen Verwendung des Ind. Perf. entspricht. „Historisch“ ist an diesem Ind. Perf. dann nur der sich auf die Vergangenheit beziehende Kontext. Es soll daher unten (Abschnitt 3.5) bedacht werden, inwiefern das hier diskutierte Phänomen nicht eher als ein Wechsel des Narrationstyps (von der späteren in die simultane Narration) verstanden werden sollte, der durch das Medium des Briefes erklärt werden kann.

Zumindest einige Vorkommnisse des Ind. Perf. scheinen allerdings „echte“ historische Perfekt-Formen zu sein, also analog zum Plsqpf. gebraucht zu werden. Am deutlichsten scheint dies beim untypischen Gebrauch des Ind. Perf. von yívo $\mu \alpha{ }^{314}$ in Gal 3,24 der Fall zu sein: V. 23 erzählt von einem früheren Zustand der

\section{GGNT 200g.}

314 Der Gebrauch des Perfekts von yívouaı im Temporalsatz in 1. Kor 13,11 scheint auf den ersten Blick ebenfalls sehr ungewöhnlich. Übersetzt wird die Verbform in der Regel wie ein Ind.

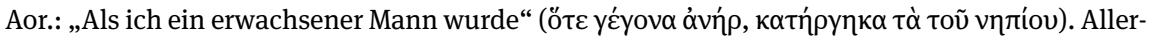

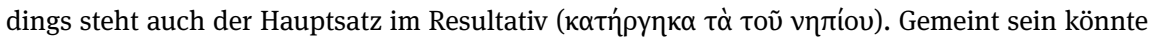
also durchaus: „Für den Zeitraum, in welchem gilt, dass ich ein Mann ,bin“ (vgl. Aristoteles, An.

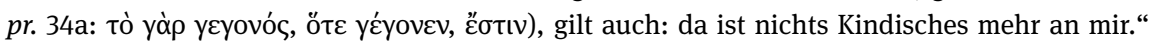
Grundsätzlich muss man dann aber wohl annehmen, dass Paulus hier von sich als einem „repräsentativen“ Menschen spricht (GGNT $255 \mathrm{f}$ wäre also auch um die 1. Sg. zu ergänzen; vgl. etwa Robertson, Grammar, 402 für andere Stellen): „Wenn man erwachsen ist, ist man nicht mehr 
Verwahrung unter das Gesetz bis zu einem damals ${ }^{315}$ noch zukünftigen Zeitpunkt

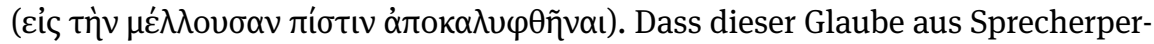

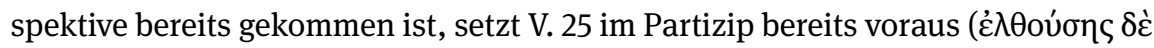

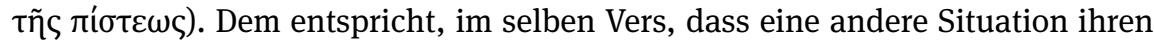
temporalen Endpunkt erreicht hat: „Wir sind nicht mehr unter dem Lehrmeis-

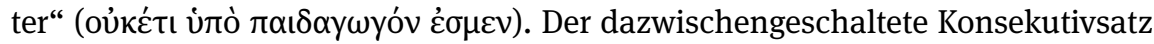
in V. 24 muss sich folglich auf eine zeitlich begrenzte Situation in der Vergangenheit beziehen: „... sodass das Gesetz unser Lehrmeister geworden war, auf

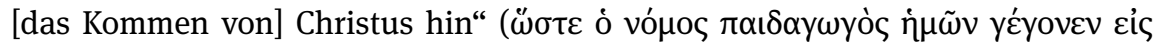
Xрıбтóv). Eine Aussage über die gegenwärtige Situation trifft Paulus hier wohl kaum, zumal wenn man દis Xpıбтóv als auf einen Zeitpunkt in der Vergangenheit verweisen sieht („,bis Christus kam“). ${ }^{316}$ Unter der Annahme, dass die Situation in V. 23 „unter dem Gesetz,“ welche im Ipf. erzählt wird (ùiò vó $\mu$ ov $\varepsilon \varphi \rho o u \rho o u ́ \mu \varepsilon \theta \alpha$

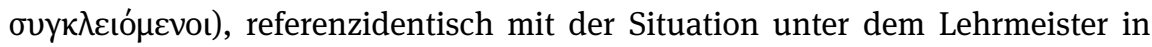
V. 24 ist, erscheint ein aoristisches Erzählen des Eintritts in diesen Zustand in V. 24 redundant. ${ }^{317}$ Im Sinn eines Plsqpf. und Äußerungsbezug der konsekutiven Konnexion ergibt der Vers jedoch einen guten Sinn: „Das heißt also, dass wir uns zu diesem Zeitpunkt in der Situation befanden, solche zu sein, welche das Gesetz als Lehrmeister ${ }^{318}$ hatten.“

\subsubsection{Aspektuelle Opposition von Indikativ Aorist und Imperfekt}

\subsubsection{Hinführung}

Die Aspektfunktion von Aorist und Durativ wird in der Koine sorgfältig differenziert. Dennoch „,begegnet man manchmal auch einem Imperfekt, an dessen Stelle durchaus ein Indikativ Aorist stehen könnte, besonders bei Verben der Bewegung

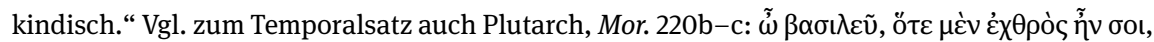

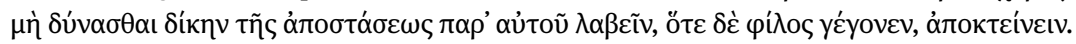

315 Vgl. GGNT 203d zur „Zukunft in der Vergangenheit“ (futurum in praeterito). Das Gesagte scheint auch für nicht-indikativische Formen von $\mu \varepsilon \dot{ } \lambda \lambda \omega$ zu gelten, wenn der Vergangenheitsbezug kontextuell deutlich ist.

316 Dann ist eine Deutung im Sinne Crellins nicht möglich. Zwar kann Paulus natürlich in der Gegenwart des Briefschreibens sagen, dass er jemand ist, für den gilt, dass er in der Vergangenheit unter dem Lehrmeister war. Diese Eigenschaft kann aber ja gerade nicht zu einem Ende kommen, wie es durch die temporale Lesart der Präpositionalphrase impliziert wäre.

317 Vgl. aber die Variante عуєveтo.

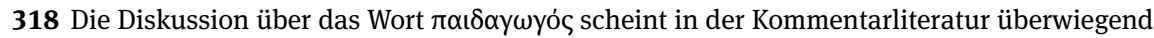
dazu geführt zu haben, dass die Merkwürdigkeit der Verbform übersehen wird. 
sowie solchen des Forderns und Fragens.“319 Bei Verben des Sprechens und der Fortbewegung ergibt sich eine zufriedenstellende Erklärung darüber, dass die entsprechende Handlung im Text noch nicht zum Abschluss gekommen ist, die anschließende direkte Rede also als Teil der Situation begriffen wird. ${ }^{320}$

Ganz grundsätzlich bedeutet die Tatsache, dass sowohl Ind. Aor. und Ipf. möglich wären (vgl. „... stehen könnte“) auch nur, dass der Situationstyp mit beiden Aspekten kompatibel ist. Auch hier können trotzdem, in reiner aspektueller Opposition, Unterschiede in der Konzeptualisierung bestehen. Immer zu beachten ist dabei die Feststellung von James: ${ }^{321}$

We may not be able either to determine or the express why an aorist has been used in preference to an imperfect, but it does not follow that there was no reason behind the decision.

\subsubsection{Aspektuelle Wahl zwischen linearem Imperfekt und komplexivem Indikativ Aorist}

Eine von der Aktionsart unberührte Gegenüberstellung der Aspekte ist, wie oben gezeigt wurde, nur sehr eingeschränkt möglich (Abschnitt 2.3.7). Tatsächliche Wahlfreiheit setzt nach Bache voraus, dass die Situation, auf welche Bezug genommen wird, als + durativ, + atelisch und + nicht-statisch gilt. ${ }^{322}$ Dies lässt in der Systematik Vendlers lediglich „activities“ bzw. die (zusätzlich als agentiv charakterisierten) Tätigkeitsverben des Dudens für freie Aspektwahl in Frage kommen.

Da atelische Situationen keinen Endpunkt enthalten, kann hier im Aorist folglich auch nicht sinnvoll einem „Abschluss“ die Rede sein. ${ }^{323}$ In diesem Sinn kom-

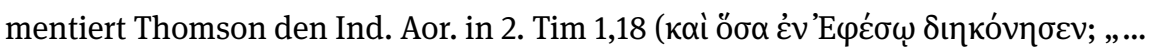
und wie viel er in Ephesus diente“):324

\section{GGNT 1981.}

320 Vgl. BDR 328-329. GGNT 1981: „Das Imperfekt wird dabei ... gern in Situationen verwendet, in denen das Angestrebte nicht oder noch nicht erreicht wird.“ Vgl. die kritische Diskussion bei Fanning, Verbal Aspect, 283-290.

321 James, „Imperfects,“ 191.

322 Bache, „Aspect and Aktionsart,“ 68. Für „gelten als“ steht im Englischen „,... is conceived of as objectively ..." Vgl. zusätzlich S. 67-77 zur (temporalen oder modalen) Distanz.

323 ,[P]erfective forms in conjunction with atelic verb constellations do not imply completion, because the situation has no natural endpoint.“ Besser sei also von „completeness“ zu reden. Horrocks, „Envoi,“ 631 noch schärfer: ,perfective forms ... of a nonterminative verb most naturally indicate that the action has merely stopped at some arbitrary point.“

324 Thomson, „Aspect,“ 63. 
The aorist tense, being perfective, expresses the totality of the situation of Onesiphorus's serving expressed by the verb constellation, but since this situation does not include an inherent endpoint, the use of the aorist does not imply that such an endpoint is reached.

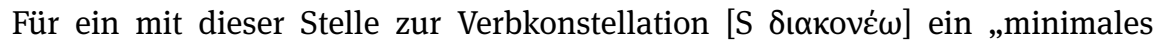
Paar“325 bildendes Vorkommnis verweist Thomson auf Mk 15,41. Hier (wie auch in Mk 1,31 - vgl. Mt 8,15 und Lk 4,39; mit DatO) liegt wohl also ein durch den Aorist in 2. Tim 1,18 nicht gegebener Fokus auf dem Dienen in seinem Vollzug. ${ }^{326}$

Man könnte also in dieser Konstellation in der Tat sagen, dass durch den Durativ als „markierte[m] Aspekt etwas grammatisch herausgestellt wird, was in der gemeinten Wirklichkeit zwar konkret enthalten ist (weil es Teil des Verbinhalts ist ... bzw. durch den Kontext signalisiert wird), aber durch (unmarkierten) Aorist sprachlich nicht speziell zum Ausdruck kommt."327

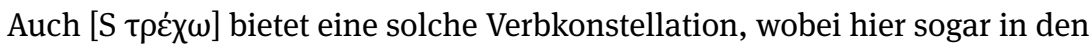
Paulusbriefen selbst ein Vergleichs-Paar vorliegt (wenn auch in unterschiedlichem Modus). In Gal 5,7 attestiert Paulus den Galatern mit linearer Betonung: „Ihr seid (doch die ganze Zeit [Ipf.])328 gut gelaufen!“329 In Phil 2,16 ist demgegenüber schlicht davon die Rede, dass Paulus „nicht umsonst ${ }^{330}$ lief.“331 Dass Paulus hier seinen gesamten Dienst vor Augen hat, ergibt sich aus dem Kontext, ${ }^{332}$ es wird die gesamte andauernde Tätigkeit jedoch komplexiv vom Aorist zusammengefasst.

325 Vgl. Bache, „Aspect and Aktionsart,“ 67 (Hervorhebung ursprünglich in Kapitälchen): „Thus it seems to me that if we want to discover the ,true nature of aspect,' not only must we minimize the purely lexical differentiation between a perfective construction and an imperfective construction, we must also minimize differences relatable to other categories, to syntax and to context: we must set up, as it were, minimal pairs with respect to aspect." Vgl. generell Bache, Study, 107-108.

326 Die Annahme eines speziell „,inchoativen“ Ipf. für Stellen wie Mk 1,13/Mt 4,11 scheint vor diesem Hintergrund unnötig. Siehe für die Kategorie grundsätzlich unten, Abschnitt 3.3.3.4.

327 GGNT 195.

328 Vgl. die Paraphrase zu Lk 22,6 in GGNT 194n: „Er suchte (über längere Zeit hinweg) nach einer günstigen Gelegenheit.“ Allerdings kommt „die eigentliche Dauer der Handlung ... bei dem Imperfekt ebenso wenig wie bei dem Präsens in Betracht“ (KG 381). Die lineare Aspektnuance macht also keine quantitative Aussage, kann aber indirekt durch die Betonung der Linearität im Kontext gegebene Aussagen über die Dauer hervorheben.

329 Menge: „Ihr hattet einen so schönen Anlauf genommen.“ NGÜ: „Ihr kamt so gut voran.“

330 Aufgrund der Präposition wäre hier eventuell das Vorliegen einer anderen Verbkonstellation (mit Zielpunkt) zu erwägen (vgl. z. B.: Offb 9,9). Siehe aber Robertson, Grammar, 550 zur Gleichwertigkeit mit Adverbien.

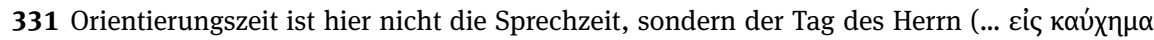

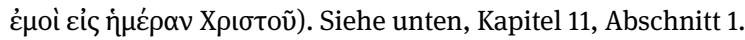

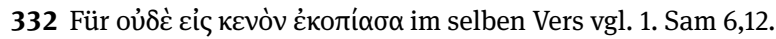


Instruktiv ist für eine Aktivität im Hinblick auf Paulus auch die Verbkons-

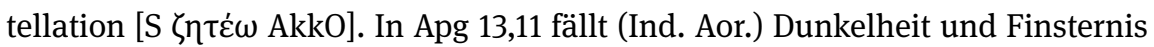

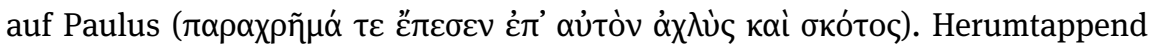

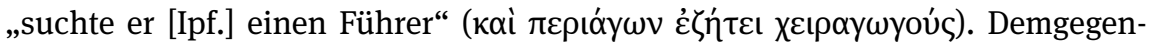
über ist in 2. Tim 1,17 über Onesiphorus gesagt: „er suchte [Ind. Aor.] mich eifrig“

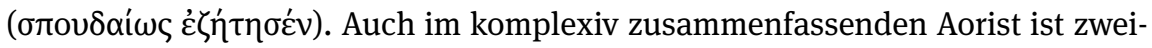
felsfrei an eine Aktserie gedacht, doch das Ipf. bewirkt die lineare Aspektnuancierung explizit durch die Form. ${ }^{333}$

Das durch das Ipf. grammatisch zum Ausdruck Gebrachte, ist freilich auch im Fall des Aorists grundsätzlich nicht ausgeschlossen - und in diesem Fall

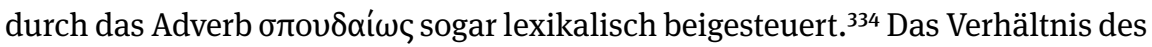
Aorists als gegenüber dem Durativ unmarkiert, was die interne temporale Struktur von Aktivitäten angeht, zeigt sich gerade an solchen beim komplexiven aoristischen Gebrauch üblichen Spezifikationen, welche meist die Dauer betreffen. ${ }^{335}$

Man kann diese häufige adverbiale Näherbestimmung als Symptom dafür betrachten, dass in diesen Fällen immer eine Uminterpretation („,coercion“) der Verbkonstellation erfolgt. Im Hintergrund steht die Annahme von Bary und Egg, dass im imperfektive Aspekt im Griechischen die Betrachtzeit eine uneigentliche Menge der Situationszeit darstellen, also auch deren temporale Begrenzungen umfassen kann, sodass die Betrachtzeit mit der Situationszeit identisch ist (anders als im obigen Entwurf von Thomson). ${ }^{336}$ Um nun Überschneidungen mit der Funktion des Aorists zu vermeiden, postulieren die Autoren eine Beschränkung der Verben, mit welchen der Aspekt kombiniert werden kann: ,we conclude that the only remaining feasible aspectual selection restriction is one of the imperfective

333 Zum selben Verb siehe GGNT 194n.

334 Wäre Onesiphorus durch das Stadttor spaziert und hätte an der erstbesten Türe klopfend direkt den Apostel gefunden, so hätte er nicht (in einem konventionell anerkannten Sinn) Mühe bei seiner Suche aufgewendet.

335 Vgl. CGCG 33.30. Armstrong, „Aorist“ argumentiert auf der Grundlage von Adverbien der unbestimmten Frequenz (mit dem Ipf. verbunden) bzw. der konkreten Anzahl (mit dem Aor. verbunden), dass der perfektive Aspekt im Griechischen für Zahlbarkeit markiert sei (S. 11). Zu

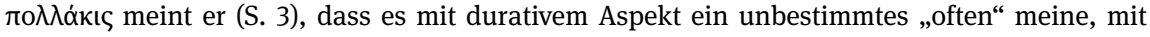
dem aoristischen Aspekt hingegen „a certain number of times“ bedeute. Es scheint aber zweifel-

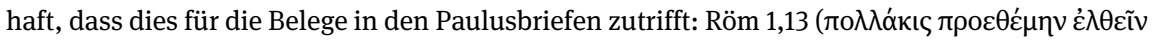

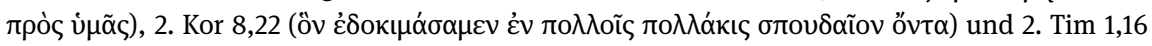

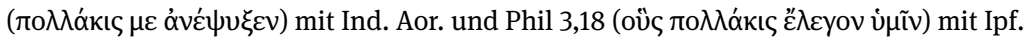

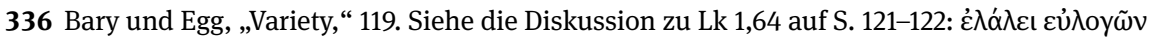
Tòv $\theta \varepsilon o ́ v$. Durch das Ipf. wird die Betrachtzeit festgelegt. Da das Partizip Gleichzeitiges zum Ausdruck bringe, sei in diesem Fall beim durativen Partizip Situations- und Betrachtzeit identisch. 
for unbounded predicates.“337 Analog wird der Aorist auf beschränkte Prädikate eingegrenzt. ${ }^{338}$ Bei der Verwendung von unbeschränkten Verbkonstellationen im Aorist beziehungsweise von beschränkten Verbkonstellationen im Durativ muss es folglich zu einer Uminterpretation kommen. ${ }^{339}$ Diese betrifft manchmal den Situationstyp (siehe oben, Abschnitt 2.3.5), immer jedoch die Darstellung der Situation mit/ohne temporalen Endpunkt. Basis hierfür ist die Differenzierung zwischen Beschränktheit („,boundedness“) und Telizität. Depreatere fasst den Unterschied wie folgt zusammen: ${ }^{340}$

A clause is telic if the situation is described as having a natural ... or an intended endpoint ... which has to be reached for the situation as it is described in the sentence to be complete and beyond which it cannot continue. Otherwise it is atelic ... A sentence is bounded if it represents a situation as having reached a temporal boundary, irrespective of whether the situation has an intended or inherent endpoint or not. It is unbounded if it does not represent a situation as having reached a temporal boundary.

Auch wenn also durch die Aspektfunktion keine Transformation des Situationstyps (entsprechend der oben explizierten Kriterien nach Vendler) erfolgt, so wird dennoch im Aorist eine temporal unbeschränkte Verbkonstellation in eine beschränkte Verbkonstellation überführt.

Umstandsangaben mit temporaler Implikation tragen hier einen wichtigen Beitrag dazu bei, das Gemeinte zu klären. ${ }^{341}$ Teilweise müssen jedoch für die Entscheidung zusätzliche kontextuelle Erwägungen herangezogen werden, wenn die Spezifikation der Dauer im Satz mit verschiedenen (beschränkten) Auflösungen des Geschehens kompatibel ist. ${ }^{342}$

Die Wahl zwischen linearem Ipf. und komplexivem Aor. kann somit also teilweise auch als unterschiedliche Konzeptualisierung des Geschehens im Hinblick auf den Endpunkt verstanden werden. Eine solche Beurteilung orientiert sich am

337 Bary und Egg, „Variety,“ 122.

338 Bary und Egg, „Variety,“119-121.

339 Bary und Egg, „Variety,“ 123-128.

340 Depraetere, „Distinguishing,“ 3. Siehe auch Liu, „End-Point Approach“ für einen neueren Überblick.

341 Bary und Egg, „Variety,“128-131.

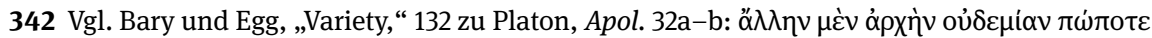

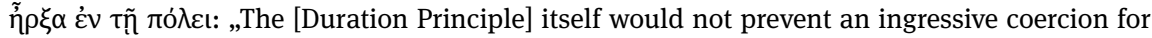
this sentence, because the topic time is the previous life of Socrates, which could encompass the runtime of Socrates' whole term as a senator just as easily as the runtime of the beginning of this term. But in the given context (Socrates having to defend himself and trying to adduce evidence in his favour), it makes much more sense to assume a complexive coercion: Only his term as a senator as a whole (and his conduct during that period) and not the mere beginning of such a term could provide evidence in favour of his personality.“ 
Unterscheidungskriterium der grammatisch spezifizierten Begrenztheit der Situationen.

Alternativ $^{343}$ zur Orientierung am im Ind. Aor. gegebenen Endpunkt kann der Unterschied in der Konzeptualisierung auch am Proprium des Ipf. eines stärkeren Fokus auf die interne Struktur der Situation verstanden werden. Nach Kühner und Gerth erscheint das Ipf. für ,wiederholte Handlung in der Vergangenheit, ein Pflegen“

nur deshalb öfter als der Aorist, weil eine wiederholte Handlung gleichsam eine zusammenhängende Reihe von Handlungen darstellt, bei der der Beschauer weit öfter den Verlauf als den Abschluss ins Auge fasst. ${ }^{344}$

Im Hinblick auf Aktivitäten kann die Aspektwahl des Erzählers also mit einem spezifischen Erzählinteresse verbunden werden: das Ipf. ist in diesen Fällen (zumindest sofern der Ind. Aor. seine Unterbestimmtheit nicht lexikalisch ausgleicht) mit einer geringen Distan $z^{345} \mathrm{zu}$ den erzählten Situationen verbunden, indem es ,a process in detail“ statt „,an event in summary““346 präsentiert: ${ }^{347}$

Wenn Begebenheiten in ihren einzelnen Momenten und Zügen in lebhafter Darstellung durch Aoriste in rascher Aufeinanderfolge erzählt werden, so wird allerdings die ganze Handlung wie ein Gemälde vor unsere Augen gerückt; allein alsdann wird das Malerische nicht durch den Aorist bewirkt, sondern liegt lediglich in der lebendigen Fassung der Erzählung ... Das Imperfekt hingegen versetzt uns mitten in die Handlung hinein und lässt dieselbe gleichsam vor unseren Augen sich entwickeln.

343 Die Definition der Aspekte über Relationen von Situations- und Betrachtzeiten ist nicht völlig unstrittig. Die ältere Metapher der Binnen-/Außenperspektive vermag manche Phänomene unkomplizierter zu integrieren (z. B. ingressiven Ind. Aor. bzw. inchoativen Ipf.; in die Gegenwart fortbestehender Ind. Aor.; Ind. Aor. mit Zustandsverben etc.). Die mit den Aspekten assoziierten Konzeptualisierungen in der Forschung scheinen nicht zuletzt mit diesen konkurrierenden definitorischen Herangehensweisen zusammenzuhängen.

344 KG 383. Hervorhebung hinzugefügt.

345 Die große „Mittelbarkeit“ des Erzählten kann narratologisch als eine stärke „Anleitung oder Einschränkung der Vorstellungsaktivitäten“ der Leser verstanden werden (Köppe und Kindt, $E$ rzähltheorie, 195). In diesem Sinn ist die Schilderung im Ipf. „anschaulicher“ (vgl. S. 193) und weist somit eine geringere Distanz auf.

346 James, „Imperfects,“ 188.

347 KG 386. Hervorhebung nicht im Original. Vgl. GGNT 1991 mit Verweis auf Apg 21,20: „[D]er Indikativ Aorist [bezeichnet] in sachlicher Kürze die wesentlichen Momente eines Geschehens ..., während das Imperfekt ... mehr bei den Einzelheiten verweilt und diese breit entwickelt und ausmalt.“ 
Diese geringere Distanz zum Erzählten kann unter Umständen als Indiz für interne Fokalisierung auf eine Erzählfigur gewertet werden. ${ }^{348}$

\subsubsection{Aspektuelle Wahl zwischen iterativem Imperfekt und komplexivem Indikativ Aorist}

Der Gebrauch des komplexiven Ind. Aor. für Andauerndes und sich Wiederholendes wird in Grammatiken häufig zusammen präsentiert, was sich auch insofern rechtfertigen lässt, als der Aorist in beiden Fällen „zwecks schlichter Nennung des Ganzen gleichsam zu einem Punkt ,zusammenfasst.““349

Allerdings besteht ein relevanter Unterschied, der die betroffenen Verbkonstellationen angeht. Das Ipf. kann keine punktuellen Situationstypen zum Ausdruck bringen. Der Ind. Aor. kann sowohl mit punktuellen Situationstypen als auch durativen Situationstypen verwendet werden. Das Ipf. in Kombination mit „achievements“ und „semelfactives“ drückt somit automatisch Iteratives aus. Der Ind. Aor. kann in diesen Fällen jedoch auch das Einzelereignis versprachlichen. Während also bei iterativer Darstellung von Verbkonstellationen mit durativer Aktionsart in beiden Aspekten kontextuelle Klärung nötig ist, ${ }^{350}$ bedarf es im Fall der Darstellung von iterativen punktuellen Geschehnissen lediglich im Aorist eine Spezifizierung.

Ein Beispiel für Letzteres bei Paulus wäre etwa 2. Kor 11,25, wo die Rede vom

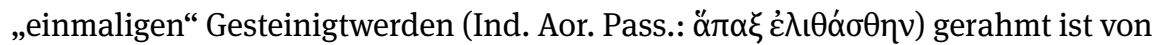
der Aussage, Paulus sei „drei Mal mit Stöcken geschlagen“ worden (Ind. Aor.

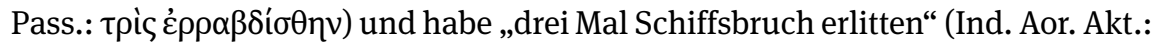

348 Bentein, „Aspectual Choice,“ 51 kommt zu folgendem Schluss: „I have argued that by choosing the aorist or the imperfect for foregrounded events, Herodotus can create a ,perspectival effect': while the aorist conveys an external perspective (the perspective of the author), the imperfect creates the illusion of an internal perspective, of a character witnessing the events." Die kritischen Aussagen zu „point of view“ und „remoteness“ (S. 49-52) als Kategorien für die Beschreibung dieses Phänomens scheinen die differenziertere Darstellung zumindest von Köppe und Kindt, Erzähltheorie nicht zu betreffen. Die Beobachtung (S. 51), dass das Ipf. entweder die Perspektive einer spezifischen Erzählfigur oder eines „virtual character“ widerspiegle, könnte eventuell dahingehend ausgewertet werden, dass das Ipf. auch als Hinweis auf die Existenz eines fiktiven Erzählers gewertet werden kann (siehe oben, Kapitel 3, Abschnitt 5.2 zur Annahme fiktiver Erzähler; vgl. dort auch Abschnitt 4.3 und 5.4).

349 Vgl. GGNT 199c.

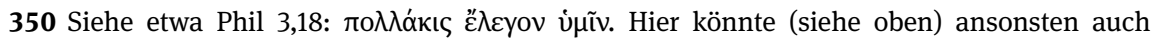

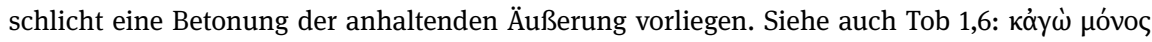

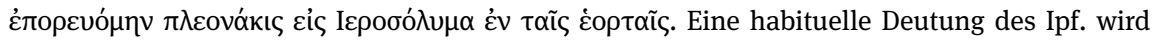
auch deutlich, wenn die kontextuell spezifizierte Betrachtzeit $z u$ lange für eine einmalige Implikation wäre. Vgl. Bary und Egg, „Variety,“ 131 im Hinblick auf Xenophon, Cyr. 8.5.10. 


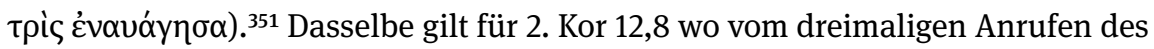

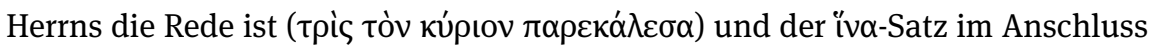
den Inhalt der dreifach zum Ausdruck gebrachten Bitte formuliert. Mustergültig im corpus Paulinum ist auch 2. Tim 1,16, wo der Autor über Onesiphorus schreibt,

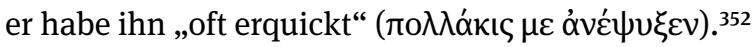

Levinsohn argumentiert, dass der Gebrauch der periphrastischen Konjugation gegenüber der einfachen Verbform eine „statischere“ Konzeptualisierung vornimmt. Dies könne die Funktion haben, Material als weniger wichtigeren Hintergrund der Erzählung zu markieren (siehe unten, Abschnitt 3.3.4 zur aspektbedingten Reliefgebung). Mit iterativem Geschehen sei die Darstellung ebenfalls „weniger dynamisch.“353 Gegenüber dem einfachen Ipf. werde die zusammengesetzte Form gebracht, ,to present iterative events, with the actor portrayed as performing the action from time to time during the period envisaged, rather than continuously.“354 Andere sind zurückhaltender, was zusätzliche Aspektnuancierungen im periphrastisch konjugierten Ipf. angeht. ${ }^{355}$

\subsubsection{Aspektuelle Wahl zwischen inchoativem Imperfekt und ingressivem Indikativ Aorist?}

Neben dem komplexiven Gebrauch des Aorists für atelische Verbkonstellationen ist auch der ingressive Gebrauch möglich (siehe oben, Abschnitt 2.2.2). Allerdings besteht hier schon deswegen keine rein aspektuelle Wahl im Vergleich zum inchoativen Imperfekt, da es dabei zu einer Verschiebung der Betrachtzeit im Aorist kommt. Ob im Durativ Stamm überhaupt eine Fokussierung auf den im Aorist exklusiv ins Auge gefassten Anfangspunkt möglich ist, ist umstritten.

Manche Grammatiken gehen von einem sogennanten inchoativen Ipf. aus, der sich auf das „Einsetzen eines länger andauernden Geschehens“ beziehe,

351 Zum Indikativ Perfekt im selben Vers siehe unten, Abschnitt 3.5 und bereits oben, Abschnitt 3.3.2.2.

352 Für eine ausführlichere Analyse dieser Textstelle siehe Kapitel 10, Abschnitt 3.2. Das Adverb in Verbindung mit Indikativ-Verbformen tritt in den unumstrittenen Paulusbriefen sowohl im Imperfekt (Phil 3,18) als auch im Aorist (Röm 1,13) auf.

353 Levinsohn, „Functions,“ 313. Von einer „Betonung“ möchte er jedoch nicht sprechen, „as the copular form may well be the default way of presenting an iterative event.“

354 Levinsohn, „Functions,“ 323. Vgl. S. 322 für sein iteratives Verständnis von Gal 1,23 und Phil

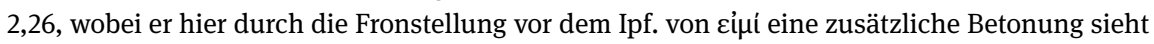

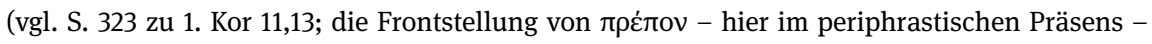
könne schlicht Standard sein).

355 GGNT 203a. Vgl. CGCGC 52.21 für „various reasons, “ die zur Wahl der periphrastischen Konjugation geführt haben könnten. 
während der ingressive Ind. Aor. auch „den Anfangspunkt bezeichn[e], aber das Andauern des Geschehens offen lasse.“356 Im Englischen entspricht dies (grob) der Gegenüberstellung von „S began doing“ und „S began to do.“357

Letztlich ist diese große Uneinigkeit wohl auf unterschiedliche zugrundegelegte Defintionen des durativen Aspekts zurückzuführen, also darauf, ob man den Einschluss der Betrachtzeit innerhalb der Situationszeit inklusive oder exklusive der temporalen Grenzen der Situation versteht. Besteht die Möglichkeit von vollständiger Deckungsgleichheit von Betrachtzeit mit der Situationszeit, so kann grundsätzlich auch eine Fokussierung auf dem Beginn der Handlung vorgenommen werden..$^{358}$

\subsubsection{Reliefgebung}

\subsubsection{Weinrichs Tempustheorie}

In der textlinguistischen Diskussion über die strukturierende Funktion der Tempora hat die Tempustheorie von Harald Weinrich eine besonders vielfältige Wirkung entfaltet, die auch die deutschsprachige Exegese erfasst hat. ${ }^{359}$ Der viel zitierte (später gestrichene) Spitzensatz „Tempus hat nichts mit Zeit zu tun“360 bringt zum Ausdruck, dass Tempora bei Weinrich primär Kommunikationssignale sind. Dabei unterscheidet er grundsätzlich zwischen „besprechenden“ und „erzählenden“ Tempora. Im Deutschen stehen sich daher Präteritum, Plusquamperfekt, Konditional I und Konditional II (erzählend) und Präsens, Perfekt, Futur und Futur II (besprechend) gegenüber. ${ }^{361}$ Dem entsprechen im Griechischen - zu dem sich Weinrich nur sehr vorsichtig (wenn auch mit sensationellen Thesen)

356 GGNT 198e. Heinrich von Siebenthal gibt eine Übersicht über die Diskussionslage in gängigen Grammatiken und entscheidet sich selbst letztlich gegen diese Kategorie.

357 Wallace, Grammar, 544-545.

358 CGCG 33.6 schließt gegenüber der Definition von Thomson, „Aspect“ etwa beide Begrenzungen (,beginning and end“) aus. Grundsätzlich scheint die in der älteren Grammatik-Tradition beheimatete Metapher der „Perspektive“ unkomplizierter mit einem inchoativen Verständnis des Ipf. verbindbar: Auch wer eine Situation „,von innen“ heraus betrachtet, wird dabei den Anfangspunkt in gewisser Weise „vor Augen haben“ - zumindest eher, als das noch ausstehende Ende der Situation.

359 Weinrich, Tempus. Siehe Zifonun, „Textkonstitutive Funktionen,“ 321-323 für eine Verortung innerhalb der gegenwärtigen linguistischen Debatten und einen Überblick über Änderungen und teilweise Abschwächungen in späteren Auflagen. Für eine aktuelle problembewusste Anwendung auf neutestamentliche Texte siehe Hoegen-Rohls, ,Johannesbriefe“ (vgl. die Diskussion oben, Kapitel 2, Abschnitt 1).

360 S. 14 der ursprünglichen Auflage.

361 Weinrich, Tempus, 30. 
äußert ${ }^{362}$ - Präsens, Perfekt und Futur (I/II) (beschreibend) und Imperfekt, Aorist und Plusquamperfekt (erzählend).

Die Dominanz einer der beiden Tempusgruppen korreliere mit einer bestimmten Sprechhaltung, mit welcher der Sprecher dem Rezipienten vermittelt, wie er sich gegenüber dem Ausgedrückten verhalten solle: Erzählende Tempora legen eine Rezeption im „Modus der Entspanntheit“ nahe, besprechende hingegen, dass der Sprecher „beim Hörer für den laufenden Text eine Rezeption in der Haltung der Gespanntheit für angebracht“ hält. ${ }^{363}$

Darüber hinaus korrespondierten die beiden Tempusgruppen mit verschiedenen Sprecherperspektiven. So seien das deutsche Präsens (besprechend) und Präteritum (erzählend) „Null-Stellen,“ die jeweils eine Nicht-Thematisierung des Verhältnisses von Textzeit (Achse des Textes in seinem Verlauf) und Ereigniszeit (Zeitpunkt-/Verlauf des Kommunikationsinhaltes) signalisierten. Die anderen Tempora brächten im Kontrast dazu ein Vorausschauen beziehungsweise Rückschauen vom Standpunkt der Textzeit aus zum Ausdruck.

Drittens - und an dieser Stelle von Interesse - könne anhand der Erzähltempora zwischen „Vordergrund“ und „Hintergrund“ unterschieden werden: ${ }^{364}$

Hintergrund ist im allgemeinsten Sinne das, was nicht unerhörte Begebenheit ${ }^{365}$ ist, was für sich alleine niemand zum Zuhören bewegen würde, was dem Zuhörer jedoch beim Zuhören hilft und ihm die Orientierung in der erzählten Welt erleichtert.

Im Griechischen liefere entsprechend das Imperfekt den Hintergrund, der Aorist den Vordergrund. Dadurch komme es zu einer Reliefgebung im Text durch die Tempora. ${ }^{366}$

Weinrich versteht die Wirkung der Tempora auch im Griechischen als von Temporalität und Aspektualität explizit losgelöst, da er beide Kategorien für

362 Vgl. die Vorbehalte bei Weinrich, Tempus, 278.

363 Weinrich, Tempus, 47. Hervorhebungen entfernt. Vgl. auch die Aufnahme in die DudenGrammatik (Duden 1836; Hervorhebungen entfernt): „Besprechende Tempora kennzeichnen eine Situation, wie sie typischerweise bei der direkten mündlichen Kommunikation vorliegt. Der Hörer prüft den Geltungsanspruch des Vorgetragenen Satz für Satz und kann, falls er bestimmte Inhalte infrage stellt, den Sprecher sofort zur Rechtfertigung seiner Äußerungen auffordern. Eine solche Diskurssituation ist auch in schriftlichen Texten anzutreffen und wird als ,gespannt‘ bezeichnet. Erzählende Tempora dagegen werden in ,entspannten` Situationen verwenden, in denen einzelne Reaktionen des Hörers oder Lesers zunächst aufgeschoben sind, da die Erzählung erst als Ganzes eine abgeschlossene Einheit bilden und dem Diskurs zugänglich ist.“

364 Tempus, Weinrich, 118.

365 So Goethe, „Goethe im Gespräch“ als Definition der Novelle. Vgl. bei Weinrich, Tempus, 146. 366 Vgl. Weinrich, Tempus, 115-120. 
unangebracht hält: „Die Aspektlehre überzeugt mich ... im Griechischen ebenso wenig wie in anderen Sprachen; ein sinnvolles System der Verbformen ist für mich von der Aspektlehre her nicht sichtbar.“367 Das Verständnis der augmentischen Sekundärtempora als „Zeitformen der Vergangenheit“ sei ebenfalls „kaum aufrechtzuerhalten.“368 Die aspektuelle Differenzierung in der Konzeptualisierung von $\beta \alpha \sigma \iota \lambda \varepsilon v ́ \omega$ im Ipf. und Ind. Aor. weigert er sich, wahrzunehmen: „Das Verb bedeutet ... in beiden Fällen: es herrschte der König.“"369

„Alle Anzeichen“ sprächen dafür - und dies tritt, wie gesagt, in expliziter Konkurrenz zur aspektuellen Differenzierung - dass sich anhand der beiden Tempora „Hintergrund und Vordergrund der Erzählung unterscheiden.“ Als „semi-finit“ bezeichnet er Konjunktiv, Optativ, Imperativ, Infinitiv und Partizip, da sie „indifferent gegenüber der Sprechhaltung“ seien. ${ }^{370}$ Die Korrelation der Stämme mit Hintergrund und Vordergrund bleibe jedoch bestehen, sodass hier die Reliefgebung ,keine bloß erzählerische, sondern eine allgemein sprachliche Reliefgebung“ sei. ${ }^{371}$

\subsubsection{Reliefgebung als Epiphänomen der Aspektbedeutung}

Diese Darstellung wird dem griechischen Verbsystem nicht gerecht. ${ }^{372}$ Die Rede von der „Reliefgebung“ greift aber dennoch eine grundsätzliche linguistische Beobachtung auf und kann auch sehr gut mit der Aspektbedeutung der Stämme verbunden werden. So nimmt auch die Cambridge Grammar of Classical Greek eine solche Vorstellung auf und formuliert: ${ }^{373}$

Aorist indicatives and imperfects are the main tenses of Greek narrative; both tenses locate an action in the past, but they differ aspectually. Imperfects, by suggesting that the action they express are incomplete (imperfective aspect), typically do not ,push a story forward': rather they are used to ,set the stage' or to create a background/framework in which main events take place which do move the story forward. These main events, in turn, appear in the aorist indicative.

367 Weinrich, Tempus, 279. Vgl. demgegenüber die Darstellung oben, Abschnitt 2.

368 Weinrich, Tempus, 279-280. Daher: „Alle Tempora des Griechischen sind zeitlos.“

369 Weinrich, Tempus, 281.

370 Weinrich, Tempus, 282.

371 Weinrich, Tempus, 283.

372 Für eine gelungene Diskussion aus exegetischer Sicht siehe Frey, Zeitverständnis, 42-49.

373 CGCG 33.49. Hervorhebung hinzugefügt. Vgl. bereits KG 386e: „Durch diesen Wechsel der Zeitformen wird der historischen Erzählung die grösste Lebhaftigkeit der Darstellung und die feinste Schattierung des Ausdruckes verliehen. Indem der Aorist die Hauptereignisse und Hauptthatsachen anführt, die übrigen Zeitformen auf verschiedene Weise Nebenhandlungen und begleitende Umstände veranschaulichend darstellen, tritt auf dem historischen Gemälde Licht und Schatten hervor.“ 
Auch Stephen H. Levinsohn ${ }^{374}$ widmet sich diesem Verhältnis in seinem Beitrag zu The Greek Verb Revisited. Für die genaue Definition dessen, was bei Weinrich „Reliefgebung“ heißt, knüpft er an die Arbeit von Kathleen Callow an, welche Vordergrund mit thematischer Information verbindet: „[It] carries the discourse forward, contributes to the progression of the narrative or argument ... develops the theme of the discourse." 375 Von nicht-thematischem beziehungsweise Hintergrundmaterial („nonthematic or background material“) spricht er ebenfalls Callow folgend demgegenüber in folgendem Sinn: „[It] serves as a commentary on the theme, but does not itself contribute directly to the progression of the theme ... [it] fills out the theme but does not develop it. “376

Vor diesem Hintergrund setzt sich Levinsohn kritisch mit Beiträgen von Stanley E. Porter ${ }^{377}$ und Robert E. Longacre ${ }^{378}$ auseinander. Beide Autoren seien sich zwar einig, dass die „storyline“ (oder „mainline“) eines narrativen Diskurses in der Koine überwiegend durch den Aorist charakterisiert sei, hätten aber grundsätzlich unterschiedliche Ansichten darüber, wie sich Imperfekt and Aorist hinsichtlich der Hintergrund/Vordergrund-Unterscheidung verhielten. ${ }^{379}$ Levinsohn gesteht zu, dass die beiden Autoren mit der Rede vom „background“ nicht exakt Gleiches meinten, sieht aber doch eine große Gemeinsamkeit: „A key feature of the approach of both Porter and Lonacre to the prominence of different tense-forms is that they equate a particular degree of prominence with individual tense-forms such as the imperfect.“380

Demgegenüber spricht sich Levinsohn für eine bloße Korrelation zwischen Tempora und dem Gesichtspunkt des ,grounding“ aus. ${ }^{381}$ Da der Aorist die Standardoption für die Darstellung eines Ereignisses als Ganzes sei, sei es in dieser Funktion unmarkiert - und daher im Kontext von narrativen Texten: Vordergrund. ${ }^{382}$ Analog gebe es sprachübergreifend ${ }^{383}$ eine Tendenz, dass ,imperfectives in narratives typically encode information of a background nature, without

374 Levinsohn, „Verb Forms.“

375 Levinsohn, „Verb Forms,“ 163 mit Verweis auf Callow, Discourse, 52-53.

376 Levinsohn, „Verb Forms,“163-164.

377 Porter, „Prominence.“

378 Longacre, „Complexity.“

379 Levinsohn, „Verb Forms,“"166-167.

380 Levinsohn, „Verb Forms,“ 167.

381 Levinsohn, „Verb Forms, “ 168.

382 Vgl. Levinsohn, „Verb Forms,“ 168 zum Konzept der Markiertheit und die Schlussfolgerungen auf S. 172.

383 Levinsohn, „Verb Forms,“ 167 zitiert Foley und Valin, Syntax, 373.397 und Hopper, „Aspect,“ 215-216. 
requiring that every event described with the imperfect in a Greek narrative be viewed as background.“384

Auch wenn Levinsohns Darstellung an diesem Punkt nur schwer zu durchdringen ist, setzt wohl auch er schlicht voraus, dass die standardmäßige Aspektbedeutung $\mathrm{zu}$ einer Darstellung von Geschehnissen führe, welche diese als „weniger wichtig“ präsentiere. Dies wird in einer anderen Arbeit allerdings deutlicher, wo er im Hinblick auf Lk 2,36-38 kommentiert: ${ }^{385}$

The above passage shows that the primary function of the imperfect in Greek is not to mark background but to portray events as incomplete. Habitual actions and other events of secondary importance in a narrative are often viewed as incomplete. It is this tendency that explains the correlation between the imperfect and background information.

Nicht kompatibel hiermit scheinen allerdings einige Beobachtungen von Levinsohn, die viel Näher an der Konzeption Weinrichs zu stehen scheinen: Werde Ipf. verwendet, obwohl dies aufgrund des kommunizierten Sachverhaltes nicht ,the natural way of portraying an event" sei, ${ }^{386}$ so sei diese Form markiert und der Leser suche nach der Relevanz des zusätzlichen „Aufwandes“ in der Verarbeitung der Form. ${ }^{387}$ Etwas überraschend expliziert Levinsohn, das Imperfekt habe dann ,added implicatures and may be backgrounded or highlighted.“388

Hier scheinen verschiedene, nur schwerlich kompatible Erklärungen vermischt zu werden. Einerseits verweist Levinsohn auf die Tendenz, dass das Ipf. aufgrund der Aspektbedeutung Geschehnisse imperfektiv darstelle, welche also wohl aufgrund ihrer z. B. gewohnheitsmäßigen Charakters oder aufgrund ihrer Unabgeschlossenheit als weniger zentral und den Hintergrund für konkrete Einzelereignisse bildend aufzufassen seien. Hier jedoch ist gerade die Situation im Blick, dass ein Geschehen nicht im Rahmen der normalen Aspektbedeutung verstanden werden kann (?) und auf diese Weise - noch dazu äußerst ambivalent (es könnte ja auch das Gegenteil bedeuten!) - eine Imperfekt-Form Reliefgebung bewirkt, ohne dass dies etwas mit Aspektualität zu tun hätte.

Demgegenüber soll hier das Phänomen der Reliefgebung aufgrund der Interaktion von Indikativ Aorist und Imperfekt ausschließlich als eine sekundäre Erscheinung der Aspekt-Unterschiede aufgefasst werden. Entsprechend relativiert sich vor diesem Hintergrund allerdings auch der heuristische Wert der Kategorie erheblich, da hierdurch nicht eine vom Aspekt unabhängige weitere

384 Levinsohn, „Verb Forms, “ 168.

385 Levinsohn, „Analysis of Narrative Texts,“ 79.

386 Levinsohn, „Verb Forms,“ 172.

387 Levinsohn, „Verb Forms,“"169 verweist hierzu auf Gutt, Translation, 103.

388 Levinsohn, „Verb Forms,“ 169. Hervorhebung hinzugefügt. 
Beschreibungsebene des Textes eröffnet wird. Die Beobachtung, dass das Ipf. in Erzählungen häufig verwendet wird, um den Hintergrund auszudrücken, unterscheidet sich daher nicht grundlegend von der Wahrnehmung, dass Verben, die von ihrer Aktionsart her Aktivitäten und Zustände ausdrücken, (übereinzelsprachlich) eine Tendenz aufweisen, den Hintergrund in Erzählungen zu liefern. ${ }^{389}$

\subsubsection{Grenzen der aspektbedingten Tendenz}

Es ist freilich keinesfalls ausgeschlossen, dass ein Erzähler gerade Zustände oder anhaltende Handlungen oder Prozesse für besonders „erzählenswert“ hält. ${ }^{390}$ Die genannte Tendenz hat folglich, sowohl was die Aktionsart ${ }^{391}$ als auch den Aspekt angeht, vor allem mit der Häufigkeit bestimmter Typen an Erzählungen (bzw. der Prominenz ihrer Kontexte) ${ }^{392}$ zu tun. Gerade bei weniger umfangreichen narrativen Vertextungen sind demgegenüber je nach dargestelltem Inhalt und abhängig von der Schwerpunktsetzung durch den Erzähler ganz verschiedene Konstellationen möglich.

Beispielsweise kann die Differenzierung sicherlich aufrechterhalten werden, wenn es sich um Erzählungen handelt, in denen ein Ereignis einen Zeithinweis bietet, mit Bezug auf welchen dann ein anderes Ereignis entfaltet wird, wie etwa in Gen 42,21: „Wir schauten über seine Seelenqual hinweg [Ind. Aor.], als er uns

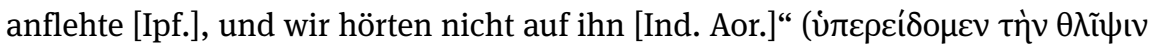

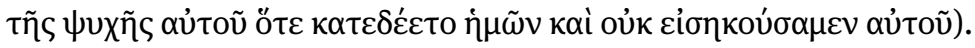

Ebenso sind aber natürlich auch Erzählungen häufig, in welchen konsekutiv die Folge eines Geschehens betont hervorgehoben wird. Die Kombination, dass der Aorist den (Hinter-)Grund zum Ausdruck bringt und der Durativ das

389 Levinsohn, „Analysis of Narrative Texts,“ 77 führt diesen Umstand an. Umso erstaunlicher ist, dass Levinsohn an anderer Stelle die Position vertritt, Ereignisschilderungen in Erzählkontexten sei mit der Grundannahme zu begegnen, dass diese vordergründige/thematische Information kommunizierten - es sei denn, dass sie explizit anderweitig markiert wären. Begründet wird das damit, dass Erzählungen ja aus Ereignissen bestünden („narratives are made up of events“): „In other words, when a narrative sentence describes an event, the default interpretation is that it is foreground. For it not to be interpreted as foreground, a backgrounding device must be used“ (Levinsohn, „Verb Forms, “ 164). Offenbar vertritt er hier ein sehr viel spezifischeres Ereignis-Verständnis. 390 Vgl. auch oben, Abschnitt 2.3.4 zu erfolgreichen und misslungenen Fluchten und dem Niederschlag der Verteilung bestimmter Handlungen in einem Korpus an Erzählungen auf der Ebene des Aspekts.

391 Siehe oben, Abschnitt 1.5.5 zu durativen Situationen als Erzählhintergrund.

$392 \mathrm{Im}$ Alltag begegnen uns beispielsweise überwiegend faktuale Erzählungen über vom Erzähler selbst Erlebtes mit informierend-feststellender Funktion (siehe unten, Kapitel 8, Abschnitt 5.2.2.2 für ein Brief-Beispiel). 
betonte Resultat, ist dann durchaus erwartbar, wenn ein singuläres Geschehen eine andauernde Folge nach sich zieht (vgl. für ein paulinisches Beispiel Röm 7,6): „Und er stand auf [Aor.] und ... ging vor aller Augen hinaus [Aor.], sodass

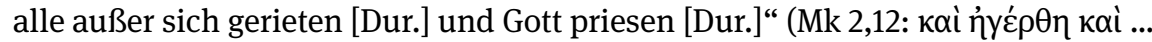

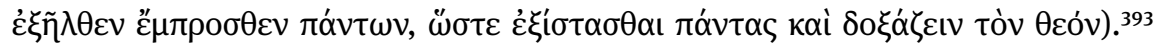
Der Aorist kann natürlich auch in solchen Zusammenhängen komplexiv wiederholtes Geschehen zum Ausdruck bringen: „... er heilte viele [Aor.], sodass alle

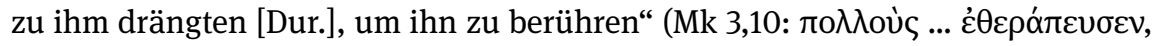

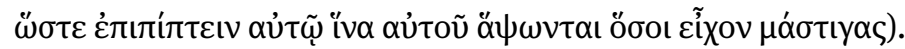

Für Erzählungen im hier definierten Sinn lässt sich folglich nicht einmal eine allgemeine Korrelation zwischen Aspekten und Reliefgebung ableiten. Es ist daher auch nicht unproblematisch, dass die Autoren in der CambridgeGrammatik sehr allgemein von „narrative“ als einem „type of text“ spechen:394

\footnotetext{
Narrative text is storytelling: it relates (usually in chronological order) a sequnce of actions that took place in the (real or fictive) past, and how they are related to each other. It normally contains a mix of imperfects/pluperfects and aorist indicatives together with less frequent historical presents.
}

Bei einem solch weiten Verständnis von ,narrative“ ist es mehr als fraglich, ob man wirklich wie folgt von einer „division of labour“ sprechen sollte: „within narrative each of these tenses [Aorist, Imperfekt, Plusquamperfekt, historisches Präsens] performs specific roles.“395 Für die sehr konkreten Erzählungen und Erzählwerke, welche die Autoren wohl eigentlich vor Augen haben, sind ihre Beobachtungen zur Reliefbildung durch das Zusammenspiel von Ipf./Aor. sehr viel unproblematischer. ${ }^{396}$

Grundsätzlich sollten Annahmen über aspektuelle Tendenzen der Reliefgebung nicht über die sich primär stellende Frage hinweggehen lassen, ob im konkreten Fall eine rein aspektuelle Wahl möglich gewesen wäre oder nicht und ob der Erzähler somit durch die verwendete Verbform eine Schwerpunktsetzung in der Konzeptualisierung der Situation vornimmt oder nicht. Versteht man kommunikative Gewichtung als ein Phänomen, das überwiegend pragmatisch bedingt ist und mit bestimmten Konnexionstypen korreliert, ${ }^{397}$ ist der Blick frei,

393 Für den Indikativ vgl. z. B. die Abfolge Ind. Aor. und Ipf. in Apg 5,40-41.

394 CGCG 33.13. Vgl. auch Smith, Parameter, 92-94 mit Warnhinweisen bezüglich einer zu simplen Gleichsetzung von Aspekt und Reliefgebung in Narrativen.

395 CGCG 33.48 .

396 Vgl. dazu den Verweis auf CGCG 61.1-3, wo die Passage Lysias, Orat. 12.5-12 (Teil einer narratio) besprochen wird.

397 Siehe dazu oben, Kapitel 4, Abschnitt 8.1. 
aspektuelle Differenzierungen innerhalb dieses so zumindest tendenziell vorgegebenen Reliefs wahrzunehmen.

Dies soll kurz anhand der konsekutiven Konnexion verdeutlicht werden. In Mk 15,5 heißt es beispielsweise: „Jesus aber antwortete gar nichts mehr, sodass

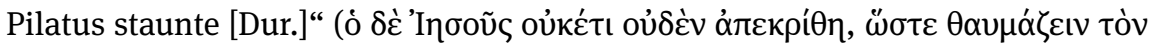

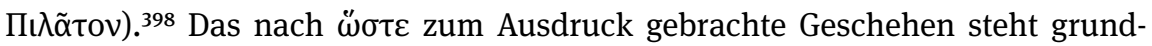
sätzlich - also völlig unabhängig von der Wahl des Stammes für den Infinitiv gegenüber dem im Hauptsatz zum Ausdruck gebrachten konsekutiven Ereignis/der Ereigniskomplex tendenziell im Vordergrund. ${ }^{399}$ Es gibt keinen Grund zur Annahme, dass ein als anhaltend markiertes Resultat weniger prominent sein sollte als es in seiner Rolle als Resultat gegenüber dem angeführten Mittel ohnehin ist (vgl. auch oben zu Mk 2,12; 3,10).

Dass die Wahl des Durativs aufgrund seiner größeren Spezifität vom Leser einen zusätzlichen Aufwand in der Auflösung des gemeinten Geschehens - der Konzeptualisierung der Situation - erfordert, heißt andererseits aber auch nicht, dass diese Wahl des Stamms die Aussage überhaupt erst in den Vordergrund holen würde. Denn auch in Mt 15,30-31 darf man wohl mit Recht davon ausgehen, dass hier ein Resultat prominent einem Geschehen gegenübersteht: „Und er heilte sie, sodass

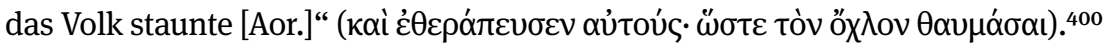

Man kann also durchaus sagen, dass der Verfasser in Mk 15,5 die Verwunderung des Pilatus „hervorhebt“ - wobei dies aber nicht im Hinblick auf den im Text (syntagmatisch) vorausgehenden Aorist gilt, sondern im Vergleich mit der an der Stelle nicht gewählten (paradigmatischen) aoristischen Form, was nämlich zu einer größeren Anschaulichkeit des Erzählten führt (vgl. oben, Abschnitt 3.3.3.2).

In Mk 4,37 wird demgegenüber zunächst der Beginn eines Sturmes im histo-

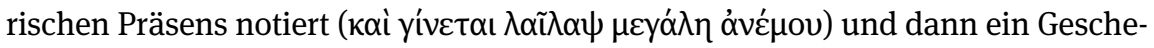

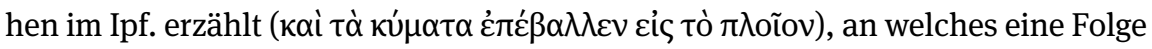

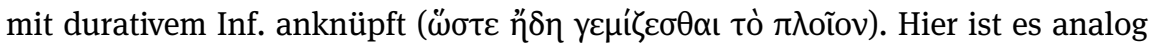
nicht das Ipf., welches den entsprechenden Hauptsatz in den Hintergrund rückt. Vielmehr ist unabhängig von der gewählten Indikativ-Form eine größere Gewichtung des Resultats - des drohenden Untergangs des Schiffes - zu erwarten. Narratologisch relevant ist das Ipf. vielmehr, weil der Autor es anstelle eines komplexiven Aorists wählt. ${ }^{401}$

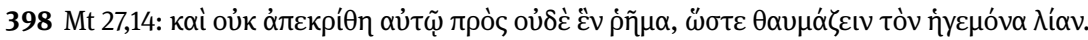

399 Vgl. oben, Kapitel 5, Abschnitt 3.4.

400 Das Beispiel wird in GGNT 334b angeführt. Vgl. auch 2. Makk 1,22: „Ein großes Feuer ent-

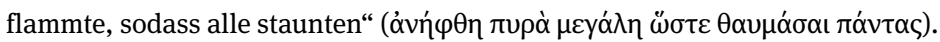

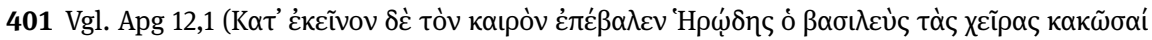

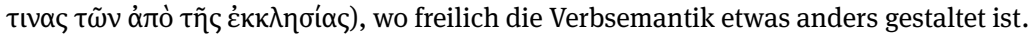


Auch für das Zusammenspiel von Aorist und Durativ - bzw. speziell auch Ind. Aor. und Ipf. - gilt daher, was Buth in The Greek Verb Revisited gegenüber den anderen Autoren in einer Fußnote festhält: „,T]he foregrounding question seems to obscure the structural choices that are being made by Greek authors." ${ }^{402}$ Die Fokussierung auf das Relief des Textes unter dem Gesichtspunkt der Aspektwahl lässt leicht relevante Beobachtungen zur Konzeptualisierung des Geschehens aus dem Blickfeld geraten.

Auch umgekehrt erscheint es im Hinblick auf die Globalstruktur des Textes viel sinnvoller, zunächst die hierarchische Propositionalstruktur zu bestimmen (und dabei auf Satzebene u. a. den Aspekt zu berücksichtigen), um dann auf dieser Grundlage anhand der herauskristallisierten Makropropositionen Aussagen über die Struktur des Textes und seine Informationsgewichtung zu treffen. Betrachtet man stattdessen schlicht einzelne Phänomene der Verbgrammatik (Modus, Diathese, Aspekt), ohne den Platz der aufgerufenen Situation im größeren propositionalen Gefüge $\mathrm{zu}$ beachten, gelangt man leicht $\mathrm{zu}$ Bewertungen, welche einer umfassenden Betrachtung der Textstruktur (vgl. auch Abb. 52) diametral gegenüberstehen. So schreibt etwa Yoon in seiner Analyse von Gal 1,11-17:403

Paul returns to background material in v. 17, with the use of $\dot{\alpha} v \tilde{\eta} \lambda \theta$ ov, $\dot{\alpha} \pi \tilde{\eta} \lambda \theta$ ov, and

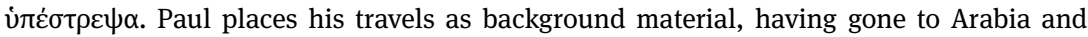
Damascus and having avoided Jerusalem. These facts are not points of emphasis in Paul's narrative, but serve to fill in the necessary details to move the story along.

Die textgrammatische Analyse dieser Passage zeigt, wie irreführend dieses auf Porter zurückgehende Analyseverfahren ist. ${ }^{404}$

\subsubsection{Grenzen der linguistische Beschreibung}

Ein grundsätzliches Problem, welches zum Abschluss zumindest noch kurz erwähnt werden sollte, stellt die im Zusammenhang mit der Diskussion der Reliefbildung durch Tempora oft nicht klare Grenzziehung zwischen grammatischer Analyse und Beschreibung interpretativer Entitäten dar. So fällt in der CGCGDefinition auf, dass „push a story forward“ und „set the stage“ in Anführungszei-

402 Buth, „Participles,“ 281. Relativ in einer Linie mit der hier gebotenen Einschätzung ist auch die Schlussfolgerung von James, „Imperfects, “ 218: „This discussion of the alternation of aorists, imperfects, historic presents, and perfects and pluperfects has demonstrated that the aspectual values of these forms are uppermost, not any indication of background or foreground that they might involve. The presence of these particles [ $\delta \dot{\varepsilon}$, oũv] suggests that the verb forms did not perform the function of marking background and foreground by themselves.“

403 Yoon, „Prominence,“ 17.

404 Siehe oben, Kapitel 5, Abschnitt 5.1.2. 
chen erscheint, wohl weil den Autoren bewusst ist, dass sie mit dieser Redeweise eher Vokabular der literarischen Interpretation aufgreifen. Analog könnte man kritisch noch weiter nachhaken, was aus der Perspektive der Grammatik denn genau mit Ereignissen gemeint sei, ,which do move the story forward“ beziehungsweise mit „main events,“ welche vorgeblich im Aorist im Text erscheinen.

Was für eine Erzählung „Hauptereignis“ ist, hängt - wie oben diskutiert (Kapitel 3, Abschnitt 5.3) - eben auch vom grundsätzlichen Verständnis der Erzählung ab, welches wiederum aufgrund verschiedener interpretationstheoretischer Vorannahmen zum Teil gewaltig divergieren kann. Ebenso können Erzählungen in unterschiedlichen Kontexten ganz verschiedene Funktionen erfüllen (siehe auch unten, Kapitel 8, Abschnitt 4), sodass ganz unterschiedliches Erzähltes im Vordergrund stehen kann. Es gibt beispielsweise keine Basis für die Annahme, Formen des Resultativ-Stamms seien aufgrund ihres „statischen Wesens“ grundsätzlich Hintergrundinformationen in Erzählungen..$^{405}$

Es ist also Zurückhaltung angezeigt, wenn die textlinguistische Diskussion der Reliefgebung durch Tempora/Stämme mit der Kategorie des Plots zusammengebracht wird, wie dies etwa im Aufsatz von Steve Runge in The Greek Verb Revisited auch terminologisch explizit geschieht, der von der im Aorist erfolgenden „plot progression“ spricht. ${ }^{406}$

Man kann hier einerseits die kritische Anfrage anbringen, ob hier nicht Kategorien, die eigentlich in den Bereich der umfassenden Interpretation literarischer Werke gehören, in die Analyse der Syntax eingetragen werden. ${ }^{407}$ Andererseits stellt die Analyse des Informationsflusses durchaus eine etablierte und sogar zentrale Fragestellung der Textlinguistik dar ${ }^{408}$ und die Frage, welche Rolle die Wahl verschiedener Verbformen für die Kommunikation von unterschiedlichen Gewichtungen von Ereignissen spielt, ist daher durchaus naheliegend.

405 Siehe etwa Levinsohn, „Verb Forms,“ 170. Vgl. Runge, „Discourse Function,“ 482 schlussfolgert auf empirischer Basis (Lk, Röm, Hebr), „that most perfects offer expositional support for a more salient element.“

406 Runge, „Grounding Status,“ 222.

407 Buth, „Participles,“ 281 empfindet wohl auch Unbehagen angesichts der Vermischung linguistischer und interpretativer Kategorien: „Although analyzing events in a text as foregrounded and backgrounded can be a useful technique as a literary analysis, I do not think that grounding itself is a broad linguistic feature of Greek grammar or syntax ... Grounding appears to be a secondary, analytical construct. This is especially the case if the definition of foreground and background becomes a confluence of semantic features of a particular event, rather than a syntactic, structural marking.“

408 Vgl. auch die Diskussion oben, Kapitel 4, Abschnitt 8.1.3. 
Der Vorbehalt ist daher aus umgekehrter Perspektive zu formulieren: Es muss darauf geachtet werden, dass in die Beschreibung der Reliefgebung anhand der Interaktion der Tempora nicht Aspekte eingetragen werden, welche umfassende Interpretationen voraussetzen. Mit der Rede vom „Plot“ kann hier beispielsweise nicht an eine gehaltvolle Konzeption gedacht sein, wie sie im erzähltheoretischen Verständnisrahmen üblich ist: Es ist eine Sache, $\mathrm{zu}$ beobachten, dass viele (/die meisten?) Imperfekt-Formen Geschehnisse zum Ausdruck bringen, welche die Haupthandlung lediglich „stützen.“409 Doch dies lässt einen immer noch mit einer großen Anzahl Aorist-Formen und der Frage zurück, ob diese denn nun allesamt vordergründig seien, in dem Sinn, dass sie den „Plot“ konstituierten. Levinsohn scheint dies zumindest nahezulegen, denn er spezifiziert als Ausnahme lediglich Aorist-Vorkommnisse in yá $\rho$-Sätzen, welche er naturgemäß für stützend hält. ${ }^{410}$ Es ist fraglich, was für die Textdeutung mit einer so immer noch sehr (!) umfangreichen „Handlung“ gewonnen wäre.

Andersherum formuliert: Es muss gefragt werden, ob Diskussionen über die Rolle von Tempora für die Reliefgebung bzw. den „grounding status“ in Erzählungen in der exegetischen Praxis nicht häufig zu viel Gewicht beigemessen wird. Einerseits wird dieses dadurch erzielt, dass mit ungenügenden Gründen über die Aspektbedeutung hinausgegangen, wird, wenn es um den Beitrag der Ipf./ Aor.-Folge für den Informationsfluss einer narrativen Passage geht. Andererseits scheint aber gerade auch der Gebrauch von Vokabular, welches ansonsten für die Beschreibung komplexer Interpretationsvorgänge Verwendung findet, das erzielte Resultat in seinen Gehalt übertrieben darzustellen.

\subsection{Erzähltempora der simultanen Narration}

\subsubsection{Der durative Indikativ Präsens als Standardtempus der simultanen Narration}

\subsubsection{Durative Aspektnuancen im Indikativ Präsens}

Standardtempus der simultanen Narration ist der Ind. Präs. Dem Aspekt entsprechend wird es (a) mit linearer Nuance gebraucht, „to refer to actions which are ongoing at the moment of speaking, “411 wobei der Beginn des Geschehens auch

409 So die bevorzugte Terminologie von Runge, „Grounding Status,“ 221.

410 Levinsohn, „Verb Forms,“ 171: „[I]n narrative, sentences introduced with yó $\rho$ never present foreground events, even when the aorist is used.“ Vgl. die Übersicht auf S. 179.

411 CGCG 33.14. 
weit in der Vergangenheit liegen kann. ${ }^{412}$ Teilweise überlappt dieser Gebrauch mit dem Englischen „ing-present.“ Dies ist etwa in 2. Kor 3,18 der Fall: „Wir werden

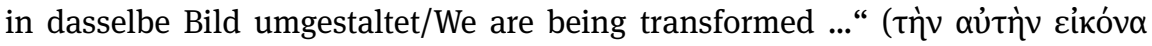
$\left.\mu \varepsilon \tau \alpha \mu \rho \rho \varphi{ }^{\prime} \mu \varepsilon \theta \alpha\right) .{ }^{413}$

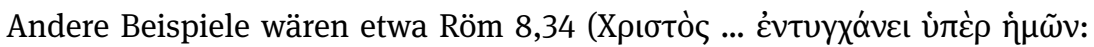

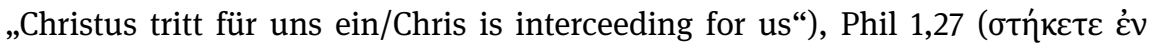

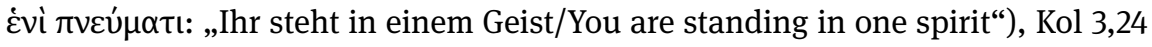

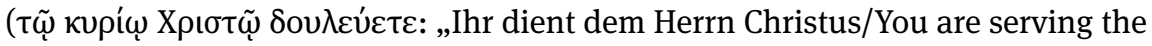

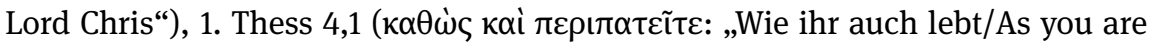
living ...“; vgl. auch 4,10 und 5,11), 2. Thess 1,4 („,... in allen Bedrängnissen, die ihr

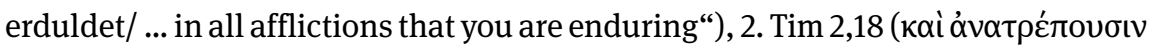

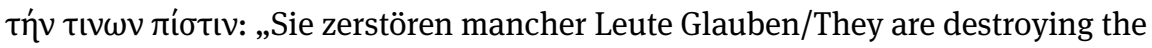
faith of some“; vgl. Tit 1,11).

Ist nicht eine andauerende Aktivität im Blick, sondern ein anhaltender Zustand, so steht aber auch im Englischen ,simple present“ (vgl. Röm 16,19: દ $\varphi$ ”

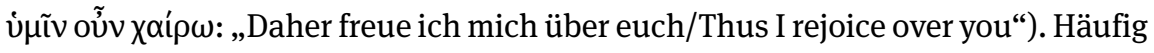
wird in Grammatiken diese Verbindung des Ind. Präs. mit Zustandsverben der Kategorie der (b) iterativen Aspektnuance zugeordnet. ${ }^{414}$ Es ist aber wohl sinnvoller, diese Kategorie tatsächlich für „repeated or habitual actions“415 zu reservieren, also für mehrfache Realisierungen der von der Verbkonstellation bezeichneten Situation, mit der Implikation, dass ,the habit is in effect at the moment of speaking." ${ }^{116}$ Teilweise ist schwer zu entscheiden, ob eine Verbkonstellation eine punktuelle oder durative Situation impliziert und ob dementsprechend eine lineare oder iterative Nuancierung vorliegt (vgl. 1. Kor 15,31: $\kappa \alpha \theta^{\prime} \dot{\eta} \mu \varepsilon \dot{\rho} \rho \alpha$

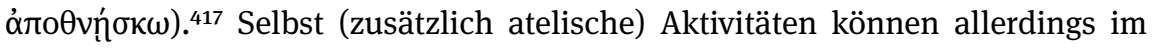
Ind. Präs. wiederholt realisiert dargestellt werden, wobei dann in der Regel eine adverbiale Markierung vorliegt. ${ }^{418}$ Beispiele für iterative Gebrauchsweisen

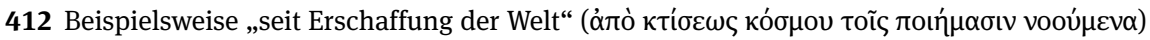

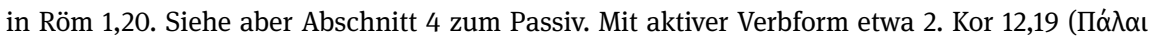

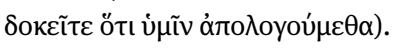

413 GGNT 197a a).

414 KG 382, die auf der „einer Handlung ..., die im Augenblicke des Sprechens sich vollzieht“ auf der anderen Seite „eine gegenwärtig wiederholte Handlung, einen gegenwärtig dauernden Zustand" gegenüberstellen.

415 CGCG 33.15.

416 CGCG 33.15.

417 Vgl. Robertson, Grammar, 827.

418 Dies ist im Deutschen nicht anders (Duden 568). Vgl. „Ich rauche“ mit „Ich stehe (gerne/seit Monaten/jeden Morgen) früh auf.“ Siehe im Griechischen etwa das linear nuancierte Beten in

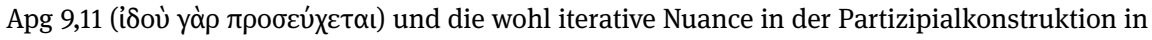




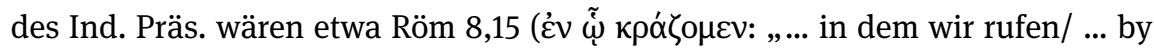

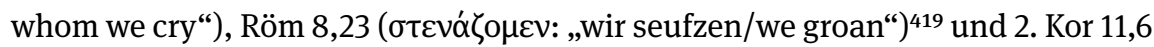

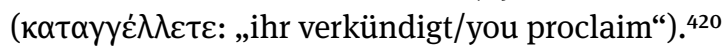

Teilweise wird hier auch zwischen iterativer und habitueller Aspektnuancierung unterschieden:421 So könnte man etwa erwägen, $\sigma \tau \varepsilon v \alpha \dot{\zeta} \omega$ als „,semelfactive“

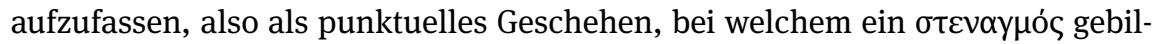
det wird. ${ }^{422} \mathrm{Im}$ Ind. Präs. wäre dann $\mathrm{u}$. U. nicht genügend Zeit, um ein einzelnes Seufzen zu bezeichnen, sodass an eine Serie an Seufzern zu denken wäre. Dies kann nun als mehr oder weniger durchgehendes Verhalten vorgestellt werden. Gestützt wird dieses Verständnis von 2. Kor 5,2, wo das Seufzen mit einem anhal-

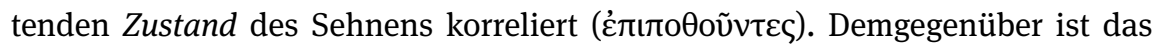

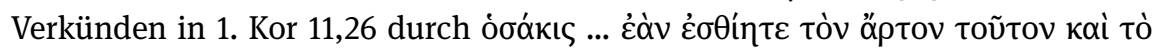

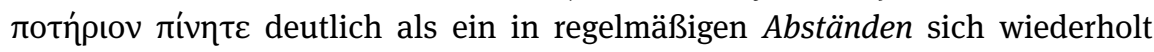
ereignendes Geschehen charakterisiert.

Freilich handelt es sich auch beim „kontinuierlichen“ Seufzen letztlich um ein „interruptatives“ Geschehen, ${ }^{423}$ die Durchgängigkeit bezieht sich lediglich auf die „relevant psychological moments“ - wie auch die Aussage „Die ganze letzte Stunde über habe ich nichts getan, als dieses Buch zu lesen.“ problemlos in diesem Sinn verstanden werden kann. ${ }^{424}$ Entsprechend sind auch die zahlrei-

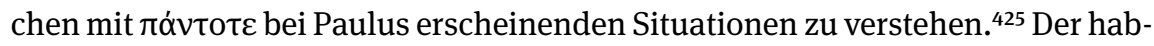
ituelle/kontinuierliche Ind. Präs. mit punktuellen Verbkonstellationen kommt dem linearen Gebrauch mit Aktivitäten sehr nahe, gerade wenn diese konzeptuell oder referentiell (vgl. Röm 1,9: „... dem ich in meinem Geist im Evangelium

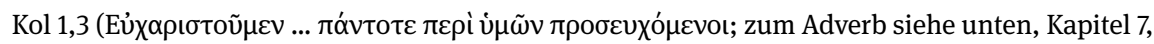
Abschnitt 4.3.3). Ohne Markierung ist teilweise nicht klar, ob das Schreiben des Gebetsinhalts selbst als ein Akt des gegenwärtigen Betens zu verstehen ist oder ob auf ein regulär stattfinden-

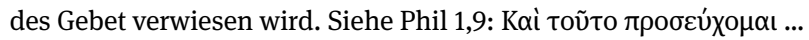

419 Eine habituelle Deutung ergibt sich hier aus der Tatsache, dass die Betrachtzeit sehr weit

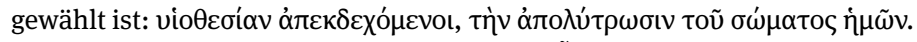

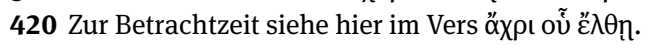

421 Z. B. Wallace, Grammar, 520-522, der allerdings 1. Kor 11,26 gerade nicht dem iterativen Ge-

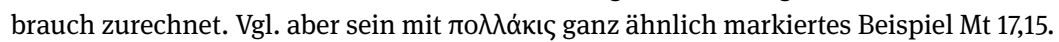

422 Allerdings scheint das Substantiv meistens auch mehr als eine Verlautbarung zu meinen und das Verb erscheint nie mit är $\alpha \xi$. Mk 7,34 könnte im Sinn eines Seufzers gemeint sein.

423 So die passende Charakterisierung bei Bache, „Aspect and Aktionsart,“ 66.

424 Vgl. Bache, „Aspect and Aktionsart,“ 65-66. Die Darstellung blendet nicht nur den zwischenzeitlichen Blick auf das Smartphone und den Gang zur Toilette aus, sondern natürlich auch sämtliche parallel laufenden physiologischen Prozesse (wie das Atmen etc.).

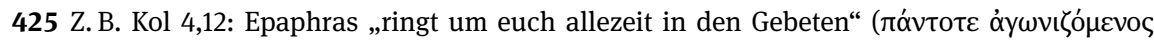
ن่rè 


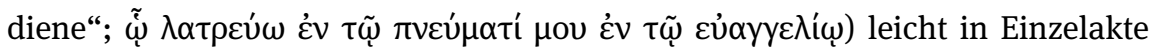
zerlegt werden können. ${ }^{426}$

In der Regel zusätzlich angenommen wird ein (c) konativer Gebrauch des Präsens: ${ }^{427}$ „With telic verbs such as $\pi \varepsilon i \theta \omega \ldots, \delta i \delta \omega \mu \mathrm{...,} \beta \dot{\alpha} \lambda \lambda \omega . . .$, the present stem may refer to an (unsucessful) attempt, because it indicates that the end-point of the action has so far not been reached.“ ${ }^{428}$ Die Nähe zum linearen Gebrauch liegt hier allerdings auf der Hand, wenn man bedenkt, dass auch die von Heinrich von Siebenthal als Beispiel für die lineare Nuance angegebene Verbkonstellation [S

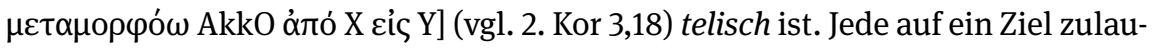
fende Situation, die nicht als wiederholt realisiert dargestellt wird, ist im Ind. Präs. im Moment der Äußerung noch nicht abgeschlossen und damit - noch nur ,versucht.“

Die konative Kategorie lässt sich für das Präsens also eigentlich nur auf zwei Weisen aufrechterhalten. Einerseits kann man sie recht weit fassen (und die lineare Nuancierung auf nicht-telische Zustände und Aktivitäten beschränken), sodass etwa auch 2. Kor 3,18 hier mit erfasst würde. Andernfalls kann man sie auch enger fassen (und die lineare Nuancierung dafür auch auf manche telische Situationen beziehen), sodass 2. Kor 3,18 nicht erfasst würde. Allerdings muss man den konativen Gebrauch dann wohl als ein lediglich kontextuell gegebenes Phänomen betrachten. ${ }^{429}$ Es handelt sich dann um eine in der konkreten Passage gegebene Voraussetzung, dass der Kulminationspunkt der telischen Situation nicht oder eventuell nicht erreicht werden wird. Dabei gibt es Grenzfälle, wie etwa

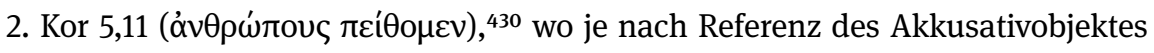
durchaus festgehalten werden kann, dass „wir (habituell) Menschen überzeugen.“

Ein Faktor, der bei telischen Verben eine in diesem Sinn verstandene konative Interpretation nahelegt, ist sicherlich auch die Diathese. So ist in 2. Kor 3,18 im Deutschen die Ergänzung mit „versuchen“ etc. weniger naheliegend, da die Konstruktion im Mediopassiv steht, also das - eventuell erfolglose - Bemühen des Agens nicht im Zentrum zu stehen scheint. Im Kontrast dazu steht in Röm

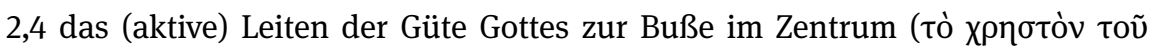

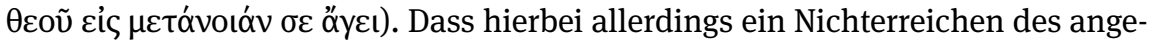
strebten Zustands vorausgesetzt ist, ergibt sich auch hier nur aus dem Kontext,

426 Im Englischen steht dann auch eher „... whom I serve.“

427 GGNT 197a c).

428 CGCG 33.17.

429 Entsprechend zurückhaltend KG 382, wobei sie dies auch auf das Ipf. ausweiten.

430 Von Wallace, Grammar, 534-535 als Beispiel einer konativen Nuance angeführt, allerdings ohne die (nach ihm nur häufige) Implikation, dass das Ziel nicht erreicht werden wird. Er ordnet hier auch Röm 2,4 ein. 
nämlich daraus, dass im Vorvers explizit gesagt wird, dass der angesprochene

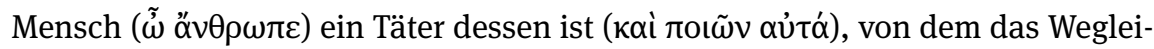
ten geschieht - also geschehen sollte.

Demgegenüber ist Gal 5,4 trotz passiver Verbform vom Kontext her ganz

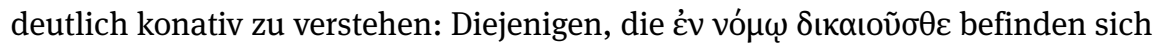
im Versuch, den Zustand der Rechtfertigung zu erlangen, doch ist diesmal eine Übersetzung mit „... die im Gesetz gerechtfertigt werden wollen“ etc. eigentlich unerlässlich, denn aufgrund von Gal 2,16 ist das Erreichen dieses Ziels als Ding

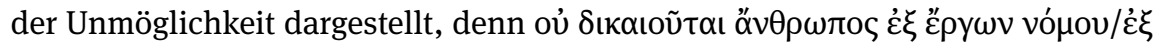

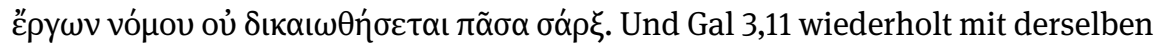

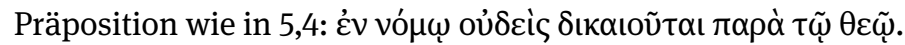

In Gal 2,14 wiederum ist die Interpretation des Vorwurfs des Paulus an

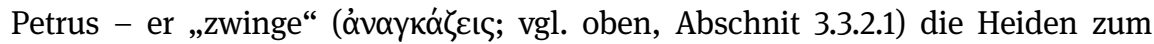

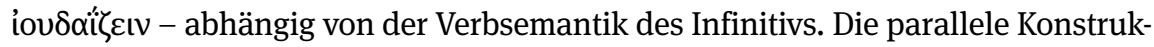

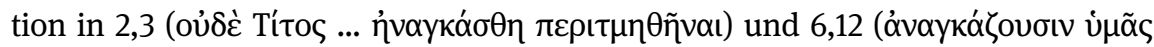

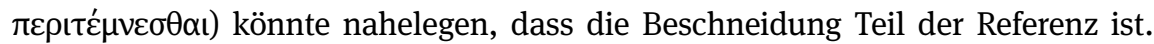
Dann wäre Petrus' Handeln ein (nicht-intentionaler) Versuch, der noch nicht zum Abschluss gekommen ist. Die Semantik des Verbs umfasst allerdings lediglich ,to customarily practice Jewish patterns of behavior.“431 Insofern man hierauf recht grundsätzlich als ,jüdische Lebensweise“ (NGÜ) Bezug nimmt (vgl. direkt zuvor

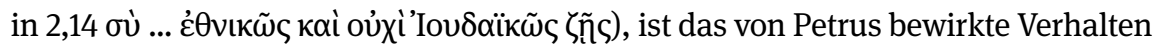
der heidnischen Mitchristen wohl auch hier noch nicht in einem solch umfassenden Sinn transformiert worden, zumal sie in der bisherigen Erzählung vollständig passiv bleiben, als alleine gelassen dargestellt werden (2,12-13). Allerdings ist die Qualifizierung als „customarily“ letztlich auch sehr graduell und wie viele Mahlzeiten in der Entwicklung in V. 13 vorausgesetzt sind und ob einzelne Heidenchristen bereits Schritte unternommen haben, um die für die gemeinsame Mahlzeit notwendige Reinheit zu erreichen, ${ }^{432}$ bleibt letztlich unklar. Es ist also durchaus möglich, dass Paulus hier im isolierten Einnehmen der Mahlzeit bereits eine - wenn auch

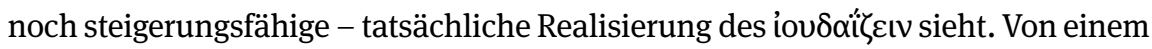
lediglich „versuchten“ Zwingen könnte dann nicht gesprochen werden.

Auch in 6,12 ist die Sachlage letztlich ganz ähnlich. Zwar läuft der Akt des Beschneidens auf einen klaren Zustandswechsel der Betroffenen hinaus. Die Vermutung liegt also nahe, dass der Zwang noch nicht zu seinem Abschluss kam, also lediglich ein Versuch vorliegt, wenn vom Zwingen in der Gegenwart die Rede

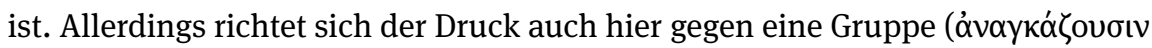

431 LN 41.32.

432 Siehe hierzu ausführlicher Heilig, „New Perspective.“ 


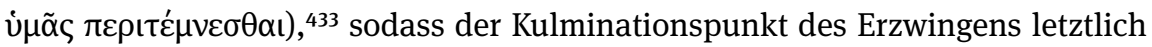
erst erreicht wäre, wenn ,ihr“ (alle?) beschnitten worden wärt. Ob einzelne Individuen bereits beschnitten worden sind, ist hier also nicht ausgeschlossen, auch wenn das (causative) ,accomplishment“ noch nicht zu seinem Abschluss gekommen ist und konativ übersetzt wird.

\subsubsection{Zur Abgrenzung von Indikativ Präsens und Imperfekt}

Der Ind. Präs. teilt sich mit dem Ipf. denselben durativen Stamm. Es ist daher nicht verwunderlich, dass es für das Augmenttempus keine großen Überlappungen mit der Gegenwartsform gibt, während der Ind. Aor. aufgrund der möglichen Aspektunterscheidung teilweise vom prototypischen in die Vergangenheit weisenden Gebrauch abweicht (s. u.).

Überschneidungen im Gebrauch ergeben sich also nicht aus einer aspektuellen Opposition, sondern, im Gegenteil, aus dem imperfektiven Charakter des gemeinsamen Stammes: Da das Ipf. das eventuelle Ende einer Situation nicht im Blick hat und der Ind. Präs. gegenwärtig Gültiges ausdrücken kann, das aber schon vor langer Zeit angefangen haben mag, können die jeweils als vergangen bzw. gegenwärtig ausgesagten Situationen oft auch wahrheitsgetreu im jeweils anderen Tempus ausgedrückt werden - was dann die Entscheidung für einen anderen Narrationstyp darstellen würde.

Diskontinuierlich wirkt eine Präsensform in der Regel vor allem im Hinblick auf die Zielsprache. So wird etwa der in 2. Kor 12,19 ausgedrückte Zusammenhang

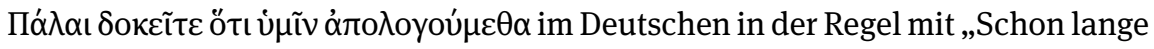
denkt ihr ..." übersetzt, ${ }^{434}$ während das Englische hierfür das „present perfect“ bevorzugt: „For a long time, you have been thinking ...“435

Unter dem Gesichtspunkt der Fokalisierung ist allerdings der Gebrauch des Ipf. an Stellen, an denen im Deutschen das Präsens bevorzugt würde, durchaus narratologisch interessant: ${ }^{436}$

Das Imperfekt scheint bisweilen statt des Präsens zu stehen, indem die durch dasselbe ausgedrückte Handlung in der Gegenwart fortbesteht. Der Redende nimmt alsdann keine Rücksicht auf das Fortbestehen der Handlung in der Gegenwart, sondern versetzt sich in den Zeitpunkt der Vergangenheit zurück, in welchem er dieselbe erkannte oder von ihr die Rede war.

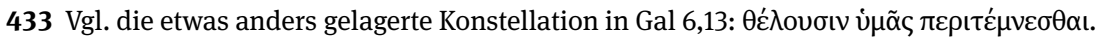

434 Duden 722: „Das Geschehen kann sich nach beiden Seiten weit über sein Jetzt hinausdeh-

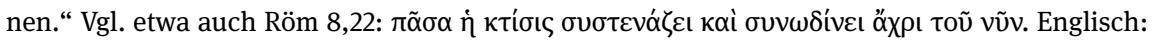
„... has been groaning ... until now.“

435 GGNT 197e. Vgl. ebenso CGCG 33.14.

436 KG 383. 
Es handelt sich dabei oft um Aussagen, die im Rahmen einer ausführlicheren späteren Narration erfolgen, wie etwa in Homer, Odyssee 3.292-293, wo zunächst

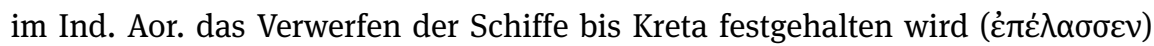
und der Ort dann spezifiziert wird als „wo die Kydonen wohn(t)en.“ So steht in

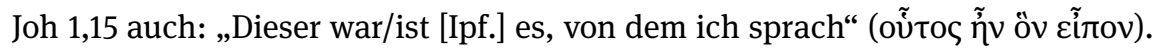

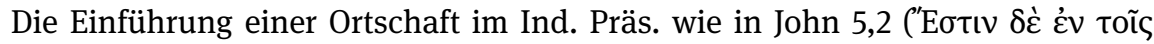

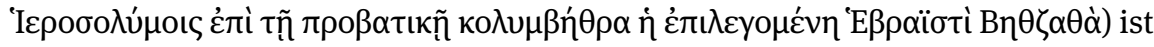
vor diesem Hintergrund als auffälliger Wechsel in die Perspektive des Erzählers $\mathrm{zu}$ werten. ${ }^{437}$

In den Paulusbriefen bedingt die häufige Thematisierung von Zuständen, die als explizit nicht-anhaltend kommuniziert werden, den recht durchgängigen

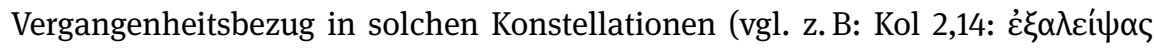

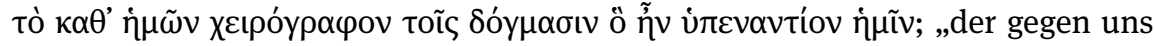
war"). ${ }^{438}$ Aber etwa in Phil 2,26 lässt sich gerade vor dem Hintergrund des brieflichen Aorists in V. 25 durchaus ein noch anhaltendes Sehnen und Unruhigsein annehmen. ${ }^{439}$ Erst danach folgt im Ind. Aor. eine komplexive Binnenerzählung

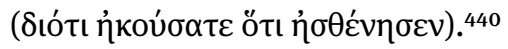

\subsubsection{Aspektuelle Opposition von Indikativ Präsenes und Indikativ Aorist}

\subsubsection{Linearer Indikativ Präsens und epistolarischer Indikativ Aorist (und Imperfekt)}

In den meisten Verbformen der 1. Sg. Ind. Präs. nimmt Paulus auf die eine oder andere Weise auf seine momentane Tätigkeit des Briefschreibens Bezug. Diese Tempus-Wahl ist aufgrund des im Moment des Schreibens ja nie schon fertigen Briefes sehr nachvollziehbar. Die lineare Aspektnuance ist etwa in 2. Kor 11,2 gut ersichtlich, wo das „Eifern“ des Paulus wohl mit seinem so eigentümlich gestal-

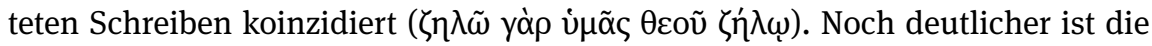
lineare Nuance, wenn Paulus durch entsprechende Verbformen auf noch zu

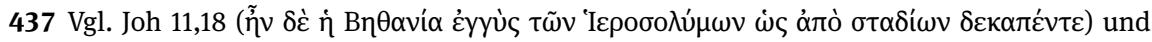

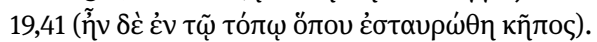

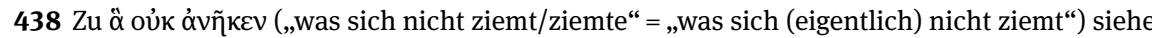
GGNT 198i.

439 Vgl. Lohmeyer, Briefe, 118: „Ich halte es für nötig, Epaphroditos ... zu euch zu schicken, da er nach euch allen verlangt und sich quält.“

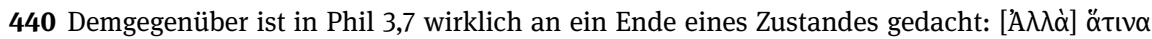

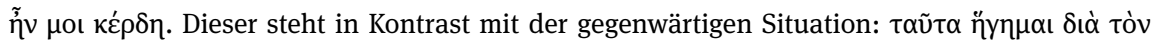

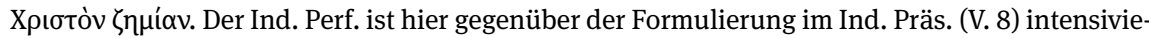
rend. Ind. Aor. $\dot{\varepsilon} \zeta \eta \mu \omega \dot{\omega} \theta \eta v$ in V. 8 markiert den Umschlagspunkt. 
Schreibendes vorausweist, da die Äußerung hier notwendigerweise noch nicht

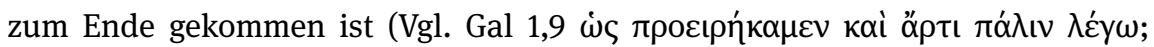

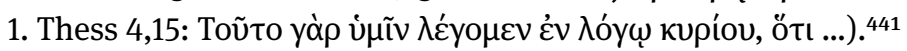

Der Gebrauch des Ind. Präs. in dieser linearen Aspektnuance überlappt mit dem „epistolarischen“ Aorist. ${ }^{442}$ Ist innerhalb eines Briefes von einem „Schreiben“ im Ind. Aor. die Rede, so liegt die Vermutung nahe, dass schlicht das bis dahin bereits Geschriebene gemeint ist. ${ }^{443}$ Teilweise zeigt sich aber an der temporalen Beziehung des Schreibers zu den Ereignissen, auf die durch den Ind. Aor. Bezug genommen wird, dass simultane Narration vorliegen muss. Besonders deutlich ist dies in Gal 6,11: „Seht, mit welch großen Buchstaben ich euch mit

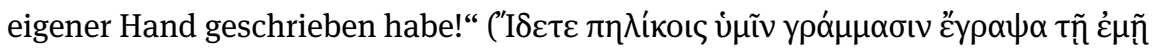

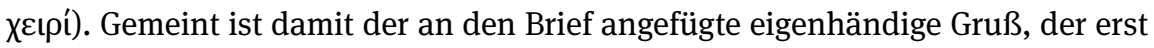
mit diesem Vers beginnt. 444

Das Vergangenheits-Tempus erklärt sich hier wohl aus der Tatsache, dass die Perspektive der Empfänger eingenommen wird. (Entsprechend kann der Schreiber im Ind. Aor. auch auf Ereignisse Bezug nehmen, die sogar noch jenseits des Abschlusses des Schreibens liegen, siehe unten, Kapitel 11, Abschnitt 1.) Letztlich korreliert die Wahl zwischen Ind. Präs. und Aor. also mit der präsentischen oder futurischen Festlegung der Orientierungszeit (dazu im Detail oben, Abschnitt 3.2).

Aus dieser primären Opposition ergibt sich sekundär in der Tat eine aspektuelle Opposition zwischen einer imperfektiven beziehungsweise perfektiven Darstellung des Schreibens. Im in den Papyri durchaus gebräuchlichen epistolarischen Ipf. hingegen fällt auch die sekundäre aspektuelle Differenzierung in der Konzeptualisierung des Schreibens weg, da auch hier der Vorgang aus der Binnenperspektive betrachtet wird. ${ }^{445}$

\subsubsection{Perfektiver/Aoristischer Indikativ Präsens und dramatischer Indikativ Aorist}

Auf das Fehlen einer aoristischen Option mit Gegenwartsbezug aufgrund der „logical imcompatibility of the perfective aspect with reference to a process or activity in process at the time of speaking“"446 wurde bereits oben (Abschnitt 2.3.2)

441 Vgl. oben, Abschnitt 3.3.3.1 zum analogen Gebrauch des Ipf. in der späteren Narration.

442 GGNT 199j.

443 Vgl. Ellis, „Aspect-Prominence,“156 zu 1. Petr 5,12.

444 Siehe nun Reece, Large Letters für Vergleichsmaterial aus den Papyri. William Varner verdanke ich den faszinierenden Hinweise, dass der Schreiber von $\mathrm{P}^{46}$ an dieser Stelle in der Tat, wenn auch kaum merklich, die Änderung in der Schriftgröße nachvollzieht.

445 Vgl. Evans, „Ghost,“ 197 für Beispiele.

446 Bache, „Aspect and Aktionsart,“ 66. 
hingewiesen. Allerdings muss einschränkend hinzugefügt werden: „speakers can refer to a single action begun and completed at (approximately) the moment of speaking." ${ }^{447}$ Heinrich von Siebenthal sieht hierin lediglich eine Variation des linearen Gebrauchs, die als eigene Kategorie für das Englische angemessen ist, da hier nicht mit „-ing present“ übersetzt wird. ${ }^{448}$

Der „instantaneous present“ 449 kommt vor allem mit performativen Sprechakten vor, wo also mit der Äußerung auch das darin enthaltene Geschen verwirklicht wird - etwa wenn Paulus in Apg 25,11 sagt: „(Hiermit) berufe ich mich auf

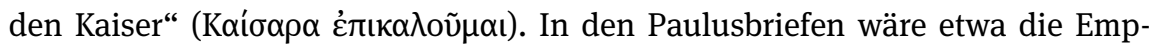
fehlung der Phoebe zu nennen, die sich dadurch ereignet, dass Paulus in Röm

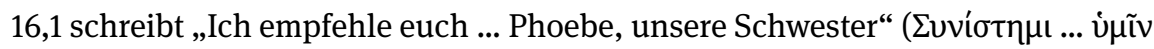

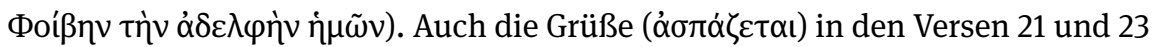
sind hier zu nennen.

In der CGCG wird dieser Gebrauch von der Situation abgegrenzt, dass ein Sprecher/Schreiber über im engeren Sinn gleichzeitige Geschehnisse Bescheid gibt, ${ }^{450}$ etwa in einer Mauerschau - wobei dann die so erfassten Ereignisse in der beobachteten Welt in der Regel bereits zu ihrem Abschluss gekommen sind. Auch im Deutschen fälllt (z. B. in Sportreportagen) „der Gipfel eines telischen Geschehens direkt in den Sprechzeitpunkt.“451

CGCG spricht hier von „simultaneous narration“ und grenzt diesen Gebrauch von der Verwendung mit performativen Sprechakten ab. Es scheint aber sinnvoller, die obigen Beispiele als Sonderfall der simultanen Narration aufzufassen, in denen also zusätzlich eine performative Dimension der Sprechhandlung zur Feststellung des gegenwärtig Geschehenden hinzutritt. ${ }^{452}$ Häufiger ist der Gebrauch des Ind. Präs. im perfektiven/aoristischen Sinn in der späteren Narration („historisches Präsens“; siehe oben, Abschnitt 3.3.2.1), wobei die Übergänge fließend sein können, da in der Regel auch die „Live“-Berichterstattung leicht verzögert geschieht. ${ }^{453}$

$\mathrm{Zu}$ einer Wahl zwischen durativem und aoristischem Stamm für die Bezugnahme auf performative Sprechakte kann es im Griechischen kommen, jedoch

447 CGCG 33.20.

448 GGNT 197a. Vgl. AGG 197a.

449 CGCG 33.20. Wallace, Grammar, 517: „aka Aoristic or Punctiliar Present.“

450 CGCG 33.20 definieren simultane Narration wie folgt: „when a speaker narrates a sequence of actions as they occur in the present.“

451 Duden 722.

452 Der zeitliche Versatz wird bei vielen performativen Sprechhandlungen „simultaner“ sein als beim Bezug auf gerade Gesehenes.

453 Vgl. „Was lässt Du meinen Blumenstrauß fallen?!“ = „Was hast Du meinen Blumenstrauß fallen gelassen?!“ 
ist der „dramatische“ Aorist ${ }^{454}$ sehr stark auf „answers and reactions in tragic and comic dialogue“ beschränkt. ${ }^{455}$ Letztlich handelt es sich hierbei auch nicht um eine Wahl zwischen verschiedenen aspektuellen Betonungen, sondern um eine Entscheidung am Festhalten an der gemeinten Zeit oder dem gemeinten Aspekt: ${ }^{456}$

The use of the aorist indicative for performatives, which are by definition perfective (by uttering the act, it is complete), makes sense given the lack of a present-tense perfective form in the tense/aspect framework of classical Greek ...: the aorist is chosen for its aspectual value, in spite of its tense. Performatives may, however, also be expressed by the present indicative ... The co-existence of these two uses suggests that either tense (pres. ind.) or aspect (aor. ind.) could be emphasized.

\subsubsection{Indikativ Präsens und Indikativ Aorist bei Zustandsverben?}

Der Gebrauch von Zustandsverben im perfektiven Aspekt stellt sprachübergreifend einen recht komplexen Sachverhalt dar. Bereits Bache weist daraufhin dass, die Unterscheidung zwischen atelisch-dynamischen Situationen und statischen Situationen oft „spekulativ“ sei. ${ }^{457}$ Mit strikt statischen Verben komme es in Sprachen, die eine solche Kombination mit dem perfektiven Aspekt grundsätzlich erlaubten, ${ }^{458}$ meist zu einer Transformation der Aktionsart, ,especially inception of the state in question." ${ }^{459}$

Ein Beispiel wäre etwa Röm 14,9, wo zunächst das Sterben des Messias

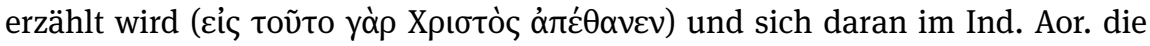

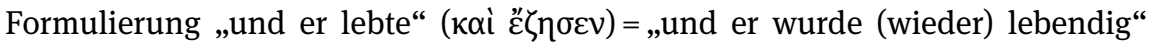
anschließt. ${ }^{460}$ Gegenüber diesem ingressivem Gebrauch ist in Apg 26,5 eine in der Vergangenheit in der Tat definitiv zum Abschluss gekommene Situation im

454 GGNT 197m.

455 CGCG 33.32. Heinrich von Siebenthal verweist auf Lk 16,4 als ein mögliches NT-Beispiel.

456 CGCG 33.32. Vgl. mit allgemeinerem Bezug Horrocks, „Envoi,“ 632: „Given that the aorist was aspectually appropriate in such situations but temporally misleading, and that the present was temporally appropriate but aspectually misleading, the emergence of a competition between the two forms in these contexts is completely predictable. Each requires an element of compromise

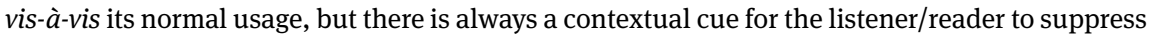
the unwanted component of meaning."

457 Bache, „Aspect und Aktionsart,“ 69. Er diskutiert als Beispiel den Satz „Er regierte für zehn Jahre. " und weist auf unterschiedliche Klassifizierungen durch Comrie und Lyons hin.

458 Für Sprachen, die eine solche Kombination nicht zulassen, siehe die Tabelle bei Bybee, Perkins und Pagliuca, Evolution of Grammar, 93.

459 Bache, „Aspect und Aktionsart,“ 69.

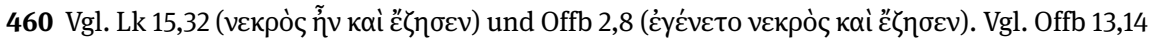
und 20,4.5. 


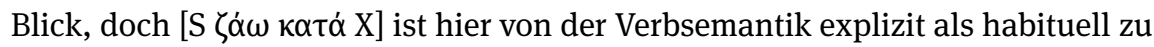
verstehen. ${ }^{461}$

Fresch räumt in seinem Aufsatz zum protoypischen Charakter des Aorist ein, dass der Ind. Aor. auch herangezogen werden könne, um vergangene Zustände

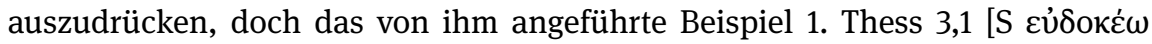
Inf.] ${ }^{462}$ demonstriert wohl eher, dass in diesen Fällen oft (vgl. eben Apg 26,4) ein weiteres nicht-statisches Situationskonzept mit der Verbkonstellation verbunden ist. ${ }^{463}$ Es ist hier vielmehr das Ipf. kurz zuvor, in 2,8, das tatsächlich ein in

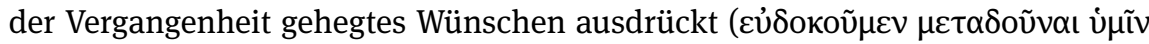

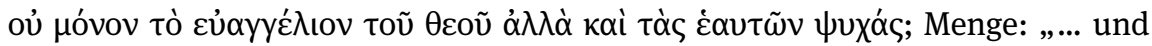
hegten den Wunsch, euch nicht nur die Heilsbotschaft Gottes, sondern auch unsere eigenen Seelen darzubringen“).

Generell ist die Beobachtung jedoch richtig, dass im Griechischen Zustandsverben im Aorist „,series of states“ in der Vergangenheit ausdrücken können. ${ }^{464}$

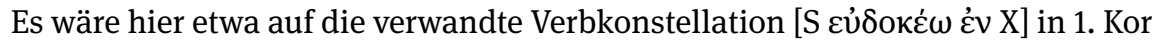
10,5 zu verweisen, wo tatsächlich der vergangene Zustand des göttlichen Wohl-

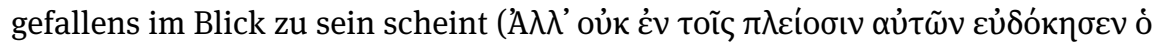

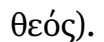

Grundsätzlich hält Fresch gegenüber diesem in die Vergangenheit weisenden Gebrauch jedoch fest, dass aus typologischer Sicht für das Griechische durchaus zu erwarten wäre, dass der Ind. Aor. in Kombination mit statischen Verbkonstellationen gebraucht wird, um einen gegenwärtigen Zustand zu kommunizieren. ${ }^{465}$ Es ist daher zu erwägen, ob das Auftreten von Zustandsverben mit Ind. Aor. als ein Phänomen der simultanen Narration aufgefasst werden kann. Es wäre dann die Unterscheidung zwischen Ind. Präs. und Ind. Aor. mit demselben Verb in der Tat vor allem aspektuell und nicht temporal-deiktisch. 466

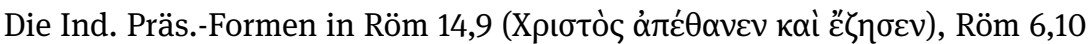

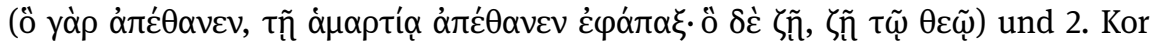

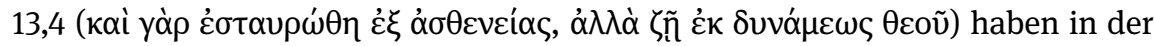

461 BDAG 3363,3: ,to conduct oneself in a pattern of behaviour.

462 Röm 15,26.27, Gal 1,15, Kol 1,19 und wohl auch 1. Kor 1,21.

463 Er übersetzt (Hervorhebung im Original): „So, not being able to endure it any longer, we were happy to be left in Athens alone.“ Vgl. ESV: „we were willing.“ Die meisten Übersetzungen entscheiden sich jedoch für das Vorliegen eines „achievement“ (vgl. auch Thomson, „Aspect,“

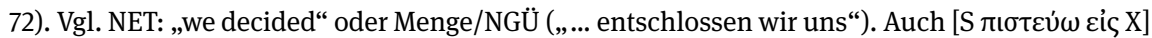
in Gal 2,16 ist wohl so zu verstehen. Vgl. auch Thomson, „Aspect,“ 72.

464 Vgl. Fanning, Verbal Aspect, 138.

465 Fresch, „Typology,“ 397.

466 Vgl. Fresch, „Typology,“ 397: „The difference ... between the aorist applied to a stative predicate and the present applied to a stative predicate is to be found in their aspectual values.“ 
Tat ein Geschehen im Blick, das in der Gegenwart anhält und im Kontrast mit einem in der Vergangenheit stattgefunden habenden Ereignis steht. Allerdings ist der in der Vergangenheit liegende Umschlagspunkt im Ind. Präs. eben tatsächlich nicht im Blick. Andersherum scheint der Ind. Aor. mit Zustandsverben - sofern hier keine in der Vergangenheit zum Ende gekommenen Zustände ausgedrückt werden - im ingressiven Aorist ausschließlich den Beginn des Zustandes auszudrücken, ohne etwas über das Andauern auszusagen.

In der traditionellen Grammatik wird ein solcher Gegenwartsbezug des Ind. Aor. (dort auch an Zustandsverben vorgeführt) entsprechend eher der Pragmatik zugeordnet: ${ }^{467}$

Das, was in der Vergangenheit eintrat, kann in der Gegenwart noch bestehen; aber dies liegt nicht im Aorist, sondern nur im Gedankenzusammenhange, wie [Homer, Ilias 1.64]

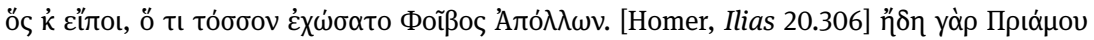

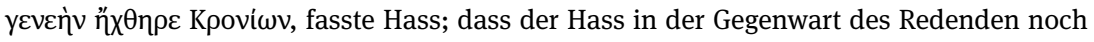
fortbestehe, liegt lediglich im Gedankenzusammenhange.

Auch Gentry ${ }^{468}$ scheint ein solches Verständnis vorauszusetzen, wenn er gegen-

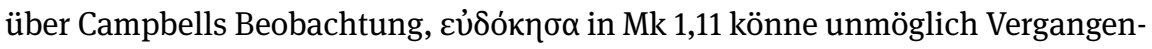
heitsbezug (,in you I was well pleased“) ${ }^{469}$ haben, meint, dieser unterscheide nicht adäquat zwischen ,semantic meaning and pragmatic effect“ und 1. Kor 1,21 als analogen Text anführt, ${ }^{470}$ wo die Übersetzung „God was pleased“ (NIV) durchaus üblich ist.

Es sollen die - wenigen - Vorkommnisse von Zustandsverben mit Ind. Aor. daher hier als Phänomen des späteren Narrationstyps verstanden werden, wobei entweder komplexiv oder ingressiv erzählt wird. Sie stehen im ingressiven Gebrauch dann allerdings auch nicht in rein aspektueller Opposition zu anderen Formen desselben Verbs. Die eigentliche Entscheidung des Erzählers findet hier also folglich zwischen verschiedenen Narrationstypen statt, nämlich zwischen simultanem und späterem.

Dieses Verständnis legt sich auch nahe, wenn man Verben, die keine Zustände, sondern punktuellere Situationen, zum Ausdruck bringen, betrachtet: Auch hier ist teilweise durchaus der erreichte Zustand als gegenwärtig vorausgesetzt (oft

467 KG 386.

468 Gentry, „Function,“ 373.

469 Campbell, Basics, 36.

470 Die Verbkonstellation in Mk 1,11 entspricht allerdings in der Tat dem eben angeführten 1. Kor 10,5! Die Kritik von Fresch/Gentry an Campbell greift hier daher zu kurz. 
sogar durch vṽv etc. markiert), ${ }^{471}$ wobei aber die Grammatik schlicht ein Ereignis in der Vergangenheit codiert. Ein Beispiel wäre etwa die Verbkonstellation

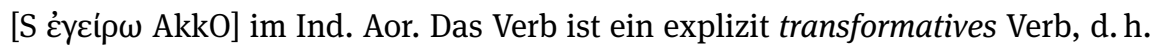
ein Verb, welches telisch ist und auf einen neuen Zustand der im Objekt ausgedrückten Entität hinläuft. Es ist hier also keine ingressive Uminterpretation nötig, um den Fokus auf einen Umschlagspunkt zwischen zwei Zuständen zu erreichen. Ein Anhalten des erreichten Zustands in der Gegenwart ist hier unzweifelhaft nicht durch die Grammatik vorgegeben. Dennoch kann dieser Aspekt kontextuell

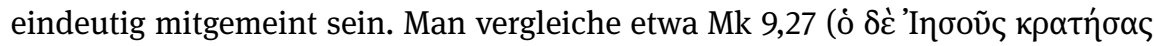

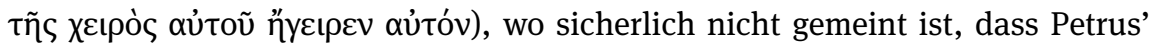

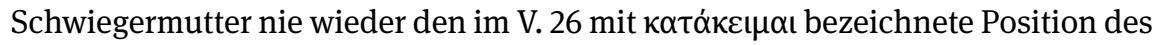
Liegens einnahm. Je nach (1) Kontext kann hier vom Übergang vom Liegen zum Stehen, vom Schlafen zum Wachen oder vom Totsein zum Leben gemeint sein. Abhängig davon, welche Art der „Auferweckung“ gemeint ist, kann nun schon aufgrund der (2) Referenz klar sein, dass damit ein Zustand erreicht ist, der nicht wieder rückgängig gemacht werden kann. Röm 6,9 bringt dies im Hinblick auf die Auferweckung Jesu explizit zur Sprache „... wissend, dass Christus, nachdem

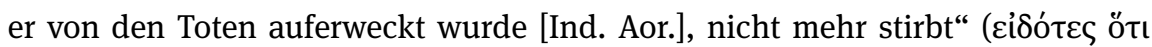

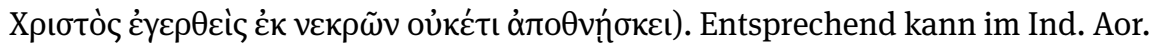
im konkreten Fall durchaus vorausgesetzt sein, dass der den Kulminationspunkt darstellende Zielzustand auch in der Gegenwart noch anhält. Dies ist etwa ganz eindeutig 1. Thess 1,10 der Fall, wo direkt im Anschluss vom zukünftigen Handeln des (offensichtlich noch immer „,auferweckten“ = „lebendigen“!) Jesus die Rede ist und bereits im Eingang des Verses gesagt wird, dass diese Person erwartet wird. ${ }^{472}$ Grammatisch zum Ausdruck kommt dieses gegenwärtige Vorliegen des Zustands explizit durch den Ind. Perf. etwa in 1. Kor 15,4.473

\subsubsection{Allgemeingültiger Indikativ Präsens und gnomischer Indikativ Aorist}

Der Ind. Präs. wird auch im Griechischen verwendet, um „eine allgemein für alle Zeiten geltende Behauptung“ aufzustellen. ${ }^{474}$ Es stellt sich hierbei natürlich die Frage, ob das sogenannte „,zeitlose“ Präsens ${ }^{475}$ überhaupt ein Mittel des Erzählens

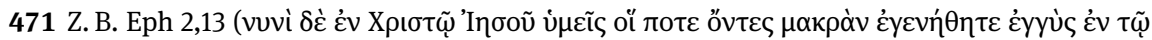

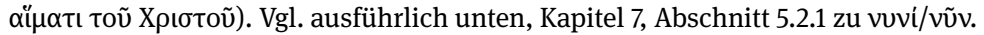

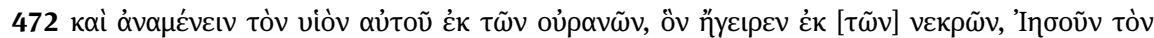

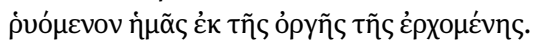

473 Vgl. GGNT 200de. Vgl. im Hinblick auf Johannes den Täufer etwa auch Mt 11,11 und Mk 6,14. Für die irreguläre temporale Bestimmung in 1. Kor 15,4 siehe aber auch unten, Abschnitt 3.5.

474 KG 382.

475 GGNT 197b. 
ist, da hier gerade etwas Allgemeingültiges ausgesagt wird, das für Gegenwart, Vergangenheit und Zukunft gleichermaßen gilt, und keine spezifische Ereignisfolge festgehalten wird.

Ein solcher Fall liegt etwa in Gal 4,1 vor: „Solange der Erbe unmündig ist,

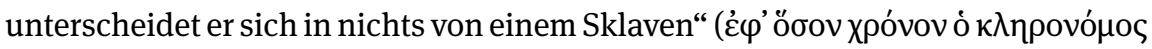

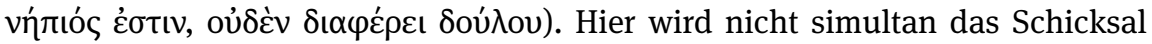
eines bestimmten Erben erzählt, sondern eine allgemein gültige Relation zweier Sachverhalte angeführt (vgl. auch etwa Röm 7,1 und 1. Kor 7,39 für denselben Temporalsatz; siehe auch z. B. Röm 8,14).

Für die Analyse bestehen grundsätzlich zwei Möglichkeiten: (1) Man kann diese Passagen als deskriptive Vertextungen auffassen, in denen eine allgemein gültige Relation festgehalten wird. Es handelt sich zwar um Prozesshaftes, doch wird das Einzelgeschehen in der Zusammenschau gleichsam zu einer statischen Beziehung. (2) Andererseits ist die „Allgemeingültigkeit“ der Relation natürlich oft auf einen bestimmten sozialen, zeitlich beschränkten Kontext begrenzt (auch wenn dies dem Sprecher unter Umständen nicht bewusst ist oder nichteinmal bewusst sein kann). So gilt etwa die Aussage zur Bindung an den lebenden Ehemann in 1. Kor 7,39 nicht universell. ${ }^{476}$ Es spricht also auch nichts dagegen, in solchen präsentisch formulierten Satzgefügen mehr oder eben auch weniger allgemeingültige Zusammenfassungen regelmäßig wiederkehrender Konstellationen zu sehen.

Entsprechend reflektiert Gal 4,1 ein Muster, das die Galater aus eigener Erfahrung in zahlreichen Fällen beobachten konnten. Man könnte folglich auch sagen, dass hier durchaus Narration vorliegt, auch wenn statt konkreten Ereignissen Ereignisbündel (iterativ) erzählt werden - und dies auf eine generalisierende Weise. Als Sonderfall der simultanen Narration werden Aussagen mit Verbformen, die (gleich in welchem Tempus) Allgemeingültiges ausdrücken hier betrachtet, da sich eine solche Verwendung „,als ein Sonderfall des Gegenwartsbezugs“ (mit zeitlich unbegrenztem Zustand) betrachten lässt. ${ }^{477}$

Analoges gilt für die im NT seltener belegten Formen des „gnomischen“ (von

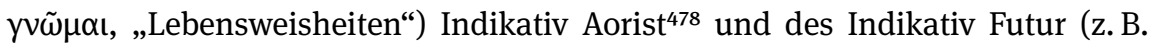
Röm 5,7). ${ }^{479}$ Ältere Grammatiken sehen den Gebrauch des aoristischen Vergangenheitstempus ohne konkreten Vergangenheitsbezug in der Regel entweder in

476 Dies gilt natürlich umso mehr bei Bezug auf Verlobte. Vgl. Schrage, Brief II, 204-205 und S. 158.

477 Duden 722 zum Präsens.

478 GGNT 1991.

479 GGNT 202i. 
einer Konzentration auf die Aspektbedeutung des Stammes oder der Verwurzlung der Aussage in Erfahrungsursachen begründet. ${ }^{480}$

Kühner/Gerth vereinigen in ihrer Erklärung beide Elemente, indem sie einerseits die Herleitung aus tatsächlichem Vergangenheitsbezug betonen, andererseits jedoch die ihrer Meinung nach recht unspezifische Aspekt-Bedeutung des

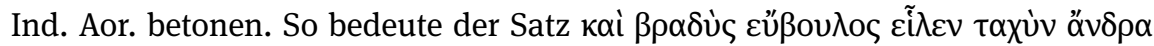

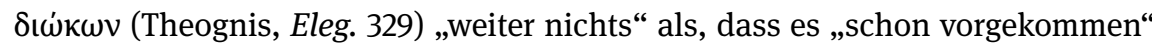
sei, dass ein langsamer, aber dabei kluger Mann den behänden Mann auf der Verfolgung eingeholt habe. ${ }^{481}$

Eine vollkommene Aufgabe des Zeitbezugs des Ind. Aor. wird dabei aber abgelehnt. ${ }^{482}$ Vielmehr tritt zur referentiellen Unbestimmtheit der auf die Vergangenheit bezogenen Aussage eine pragmatische Komponente: „Dass es gegebenenfalls jederzeit wieder vorkommen kann, ist nicht ausgesprochen, wird aber vom Hörer ebenso wie vom Redenden hinzuergänzt.“ Es liegt also kommunikativ das „Hauptgewicht auf der Nutzanwendung für die Gegenwart oder Zukunft,“ wobei dies einerseits unausgesprochen bleibt, andererseits „dem Redenden aber in Gedanken vorschwebt.“

Nach wie vor plausibel ist an dieser Einschätzung, dass die „Wahrheiten und allgemeine Urteile“ letztlich „,auf Erfahrung gegründet sind, sowie Erscheinungen, welche in der Vergangenheit öfters wahrgenommen sind“ darstellen. ${ }^{483}$ Schließlich haben Generalisierungen zumindest indirekt eine Beziehung zu vergangenem (und gegenwärtigem und zukünftigem) Geschehen:

A structural generalization is of virtual character: it involves a mental construction of a generic event type. A generalization, however, also indirectly invokes actual instantiations (tokens) experienced in the past. The generalization is, after all, based on the observation of structural similarities between the actually experienced events. A structural generalization also has a future dimension: a generalization can be employed to project the occurrence of similar events in the future.

Der Gebrauch des Vergangenheitstempus für Allgemeingültiges lässt sich also in der Tat gut aus dem auch die Vergangenheit betreffenden Anspruch erklären, tatsächliche Erfahrungen aufzugreifen. ${ }^{484}$

480 Fresch, „Typology,“ 382-384.

481 KG 386.

482 KG 386, Fußnote 7.

483 KG 386.

484 Vgl. Allan, „Tense,“ 99-100, der den (von KG nicht vom „gnomischen“ Gebrauch differenzierten) empirischen Aorist als Bindeglied betrachtet. 
Ebenso wie der irreale Gebrauch der Augmenttempora stellt die so erklärbare kontextspezifische überzeitliche Verwendung des Ind. Aor. letztlich eine mit der prototypischen temporalen Bedeutung gut verträgliche Entwicklung dar: In allen drei Fällen ist das Geschehen der direkten Erfahrung von Sprechern und Hörern entzogen. Diese nur indirekte Zugänglichkeit stellt eine Distanz dar, die im prototypischen Fall temporal interpretiert wird, im kontrafaktischen Fall als epistemische Kluft und im Fall des gnomischen Ind. Aor. als eine Distanz, die darin begründet ist, dass das genannte Ereignis nicht direkt wahrnehmbar ist, sondern der Verweis auf ein ,virtual event epistemically at a distance from reality“ erfolgt. ${ }^{485}$

Aus narratologischer Perspektive fällt im Hinblick auf den Ind. Aor. also einerseits die große Nähe zu Erzählungen konkreter Ereignisse auf, andererseits aber auch der letztlich hypothetische Charakter. ${ }^{486}$ Beide Elemente kommen zusammen, wenn im Griechischen sogar der Erfahrung grundsätzlich nicht zugängliche Ereignisbündel der Anschaulichkeit wegen im Ind. Aor. dargestellt werden. ${ }^{487}$

Gegenüber der Annahme von Kühner/Gerth kann vor diesem Hintergrund am grundsätzlich geltenden Vergangenheitsbezug des Ind. Aor. festgehalten werden, ohne diesen auch im gnomischen Gebrauch vorausgesetzt zu sehen. Vor dem Hintergrund des prototypischen Charakters des Ind. Aor stellt sich der Gebrauch in Kontexten ohne Vergangenheitsbezug schlicht als nicht im Widerspruch zu einer prototypisch grammatisch codierten absoluten Zeitbedeutung dar. ${ }^{488}$

Korrekt erfasst wird von Kühner/Gerth jedoch, dass der Parameter der Allgemeingültigkeit nicht primär durch die gewählte Verbform kommuniziert wird, sondern in der Regel kontextuell gegeben ist (womit die Wahl des Stamms

485 Allan, „Tense,“ 97.

486 Vgl. Horrocks, „Envoi,“ 632: „In other words, in these unreal, i. e., atemporal and future/ hypothetical, domains, the relevant occurrence is conceptualized in such a way as to require the use of a perfective present, even though the event in question never takes place in the actual, real-world present.“

487 Vgl. KG 386: „Selbst in der Anführung von Dingen, welche nicht aus der Erfahrung, sondern nur aus der Phantasie entnommen sind, gebraucht der Grieche, um dieselben zu versinnlichen und zu veranschaulichen, den Aorist.“

488 Fresch, „Typology,“ 404: „The fact that the aorist indicative can occur in nonpast contexts in order to express a gnomic idea, a present state, a performative action, or a future event is not evidence that the aorist indicative is not semantically encoded for + PAST. It simply demonstrates that it is polysemous. What I am claiming is not all that different from the historical grammarians. Their intimate knowledge of the Greek language often led them to accurate conclusions even if they did not have the linguistic theory to back it up. The only difference may be that while they viewed these nonpast aorist indicatives as exceptions to the rule, I view them as typologically expected and pragmatically or lexically motivated deviations from the prototypical usage.“ 
letztlich aspektuell zu erklären ist). ${ }^{489}$ Gegenüber dem „allgemeingültigen“ (auch teilweise „gnomisch“ genannten) Ind. Präs. lässt sich dann auch eine recht weitgehende Austauschbarkeit feststellen. Die CGCG hält jedoch fest, dass für „truly ,timeless“ truths“ lediglich das durative Tempus gebraucht werde ${ }^{490}$ (wobei der Umkehrschluss wohl nicht gezogen werden darf). ${ }^{491}$ Dies spiegelt wohl die Tatsache wider, dass der gnomische Aorist auf telische Verben beschränkt zu sein scheint $^{492}$ (und somit die Äußerung von Zuständen der durativen Form vorbehalten bleibt).

In der immer noch großen Schnittmenge stellt die Entscheidung zwischen Ind. Präs. und Ind. Aor. somit tatsächlich eine Differenzierung ausschließlich in der Konzeptualisierung der Situationen dar:493

The contrast between a gnomoc aorist and a generic present can also be described in terms of construal. The gnomic aorist focuses the attention on the fact that each individual instance (subevent) out of all potential iterations is bounded. For example, in $\alpha$ $\lambda \lambda \omega \mu \varepsilon \dot{v} v$ yò $\rho$

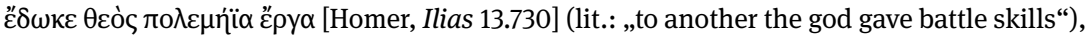
the aorist signals that each individual instance of the act of giving is viewed as bounded. In the second way of construal - involving a generic present i. e., imperfective aspect) - , the speaker defocuses (,zooms out“) from the individual subevents and conceptualizes the series of subevents as a higher-order macroevent. The speaker does not wish to be specific about the internal structure of the subevents but construes the chain of subevents as a macroevent without initial or final boundaries: the open-ended chain of iterations is regar-

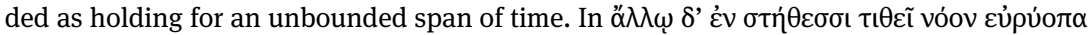

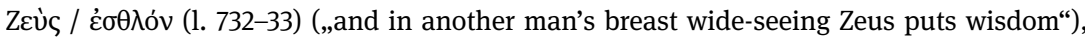
for example, the present (imperfective) aspect conveys that the series of iterations extends indefinitely into the past and into the future.

Vorkommnisse des gnomischen Ind. Aor. in den Paulusbriefen sind meist strittig und können oft anderweitig erklärt werden. In Röm 3,23a folgt die Aussage „Denn

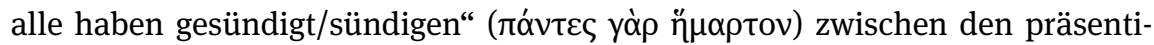

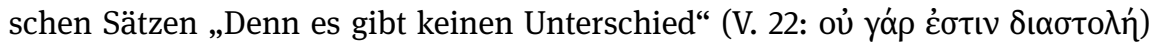

489 Allan, „Tense,“ 93.

490 CGCG 33.31. Vgl. KG 386: „Auch die griechische kann [den Ind. Präs.] gebrauchen, und gebraucht sie regelmässig, wenn ein zu allen Zeiten absolut gültiges und entweder auf Vernunftgründen beruhendes oder durch häufige Erfahrung zur allgemeinen Wahrheit gewordenes Urteil, oder Sitten und Gewohnheiten, welche in der Gegenwart des Redenden fortbestehen, angeführt werden.“

491 KG 386: „Aber auch in Erfahrungssätzen von nicht absoluter Gültigkeit wird sehr häufig die Präsensform wie im Deutschen gebraucht.“

492 Vgl. Allan, „Tense,“ 92 und CGCG 33.31.

493 Allan, „Tense,“ 92-93. Vgl. GGNT 1991: Der gnomische Aorist ist „im Gegensatz zum zeitlosen Indikativ Präsens nicht durativisch.“ 


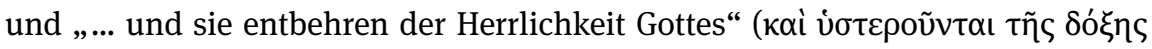

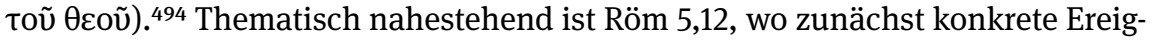

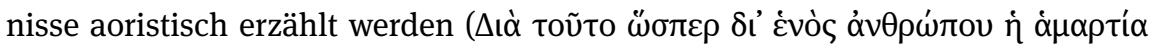

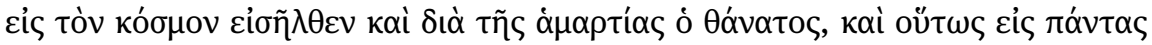

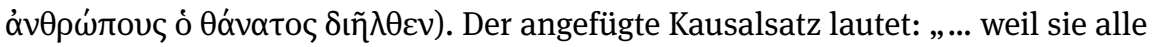

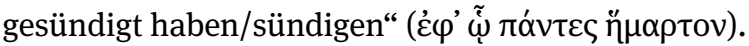

Dass schlicht komplexiv Ereignisse zusammengefasst werden, ist in der zuweilen auch angeführten Passage Röm 6,4-6 schon aufgrund der 1. Person Pl.

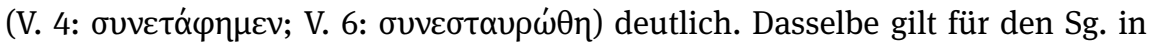

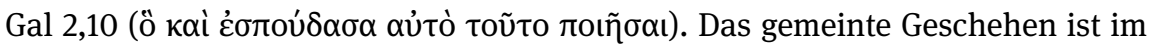
ersten Fall durch mehrere Personen verwirklicht und im letzten Fall eine lange andauernde Realisierung, gemeint sind jedoch konkrete Situationen in der Vergangenheit, die dann im Aor. lediglich zusammengefasst werden. ${ }^{495}$

In Röm 8,30 wird für die abschließende „Verherrlichung“ der Aktreihe (oü $\delta \dot{\varepsilon}$

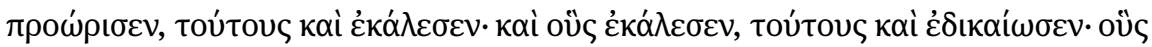

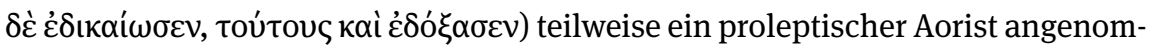
men. ${ }^{496}$ Denkbar wäre allerdings auch ein gnomischer Gebrauch im gesamten Vers. ${ }^{497}$ Auch für 1. Kor 15,49 wird erwogen, ob hier von einem zeitlosen „Tragen“ die Rede ist oder proleptischer Gebrauch vorliegt. ${ }^{498}$

Tatsächlich plausibel ist 2. Tim 2,19, wo vom durch Gott etablierten Funda-

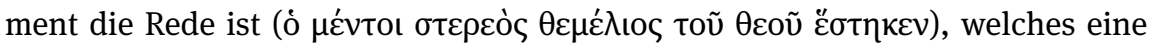

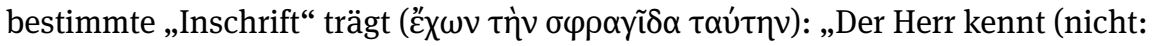

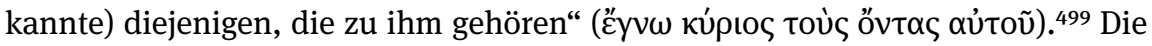
allgemein gehaltene Adressatenschaft im zweiten (imperativischen) Teil bestätigt diese Lesart: „Wer sich zum Herrn bekennt, der trenne sich von allem, was

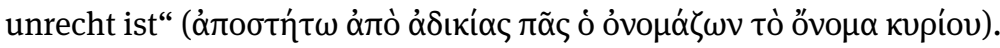

494 Robertson, Grammar, 837.

495 Vgl. demgegenüber Jak 1,11. Vgl. aber auch GGNT 1991.

496 Wallace, Grammar, 564.

497 So Robertson, Grammar, 837.

498 NSS 995. Vgl. zum anhaltenden (klar linear nuancierten) Herumtragen 2. Kor 4,10 (...

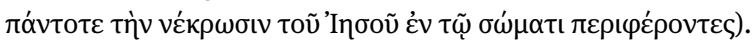

499 Im ursprünglichen Kontext in Num 16,5 ist der Ind. Aor. mit einer Ind. Perf.-Form koordiniert

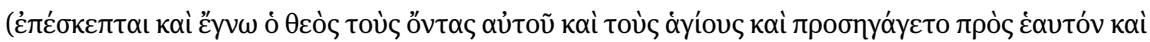

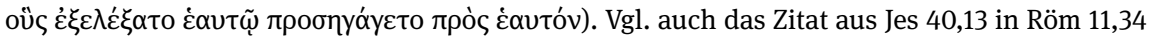
und 1. Kor 2,16. Vgl. zu LXX-Zitaten GGNT 1991. 


\subsubsection{Aspektuelle Opposition von Indikativ Präsens und Indikativ Perfekt}

\subsubsection{Resultativer Indikativ Präsens und Indikativ Perfekt}

Im Fall des epistolarischen Aorist führt eine Verschiebung der Orientierungszeit dazu, dass der Ind. Aor. Bezug nehmen kann auf ein Ereignis, dessen Situationszeit mit der Sprechzeit koinzidiert, das Vergangenheitstempus also anstelle des Ind. Präs. verwendet werden kann.

Umgekehrt tritt der Ind. Präs. allerdings an einigen Stellen auch im Hinblick auf bereits Geschriebenes auf. Gerade im Fall von y $\rho \dot{\alpha} \varphi \omega$ wählt Paulus häufig den Ind. Präs. obwohl er auf Teile des Textes Bezug nimmt, die bereits abgeschlossen

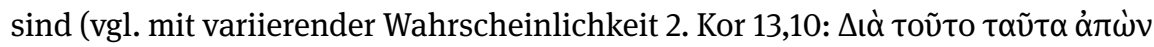

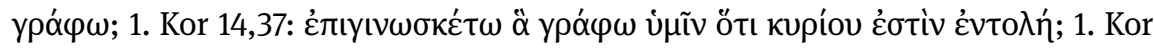

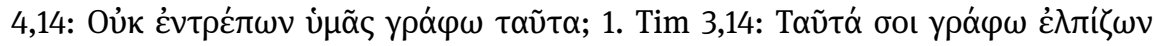

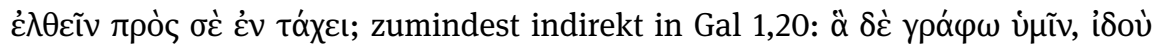

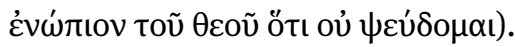

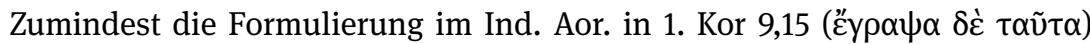
scheint zurückzuverweisen, nimmt also das erfolgt habende Schreiben aus der aoristischen Außenperspektive wahr. ${ }^{500}$ Letztlich scheint der Ind. Präs. hier aber

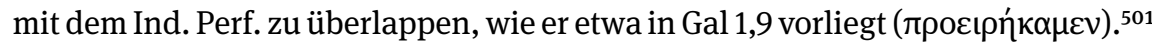
Besonders deutlich ist der Rückverweis innerhalb desselben Schriftstücks in 2. Kor 7,3: „Ich habe ja schon vorhin gesagt, wie sehr ihr uns ans Herz gewach-

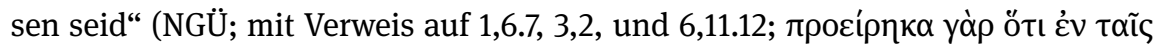

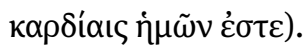

Dieser „resultative“ Gebrauch des Ind. Präs. ist auf einige wenige Verben

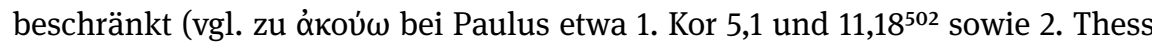
3,11). Es ist fraglich, ob hierfür tatsächlich eine vom linearen Gebrauch verschiedene Kategorie angenommen werden sollte. Auch wenn Demonstrativpronomen etwa auf bereits „Geschriebenes“ zurückverweisen, ist doch zugleich der Prozess des „Briefschreibens“ nicht abgeschlossen. Diese verschiedenen Situationszeiten

500 Ein duratives Gegenstück gibt es bei Paulus anscheinend nicht. Vgl. lediglich 2. Kor 9,3 (wo

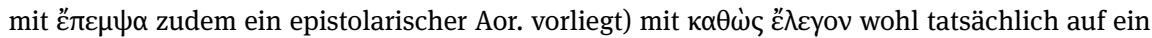

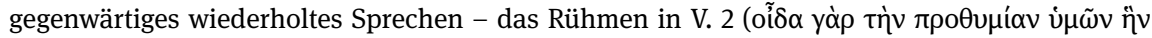

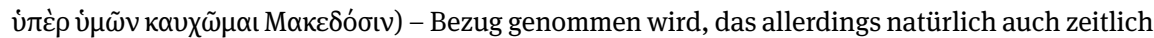
bis vor den Brief zurückreicht.

$501 \mathrm{Vgl}$. CGCG $33.18 \mathrm{zum}$,use of the present indicative of some verbs of hearing, learning, saying etc., to refer to the content of an earlier speech or message.“

502 Paulus setzt hier interessanterweise die simultane Narration im Ind. Präs., seine Reaktion

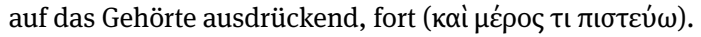


kommen in dieser spezifischen Kommunikationssituation vermutlich zusammen und kreieren den Eindruck eines „untypischen“ Gebrauchs. ${ }^{503}$

Der etwas anders gelagerte Fall von telischen Verben, bei denen im Altgriechischen zuweilen neben dem Ereignis selbst auch das anhaltende Resultat im Blick sein kann, ist bei Paulus nicht belegt. ${ }^{504}$ Bereits von der Verbsemantik her

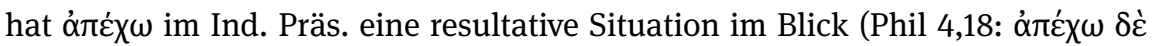

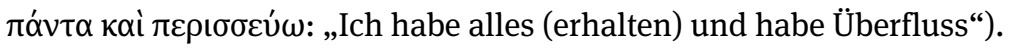

\subsubsection{Rein aspektuelle Wahl bei analogen Zustands-Situationen}

Eine rein aspektuelle Wahl zwischen Ind. Präs. und Ind. Perf. trifft ein Sprecher im Hinblick auf die Ausgestaltung einer Verbkonstellation, wenn diese in beiden Fällen einen Zustand ausdrückt (siehe Abb. 48) - wobei dann wohl von einer intensivierenden Aspektnuancierung im Resultativ auszugehen ist:

\begin{tabular}{|c|c|c|}
\hline & Ind. Präs. & Ind. Perf. \\
\hline 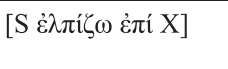 & 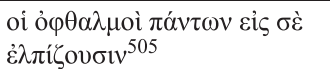 & 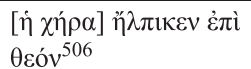 \\
\hline$\left[\mathrm{S} \dot{\varepsilon} \lambda \pi \mathrm{i} \zeta \omega \varepsilon \dot{\varepsilon} \varsigma^{507} \mathrm{X}\right]$ & 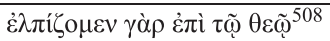 & 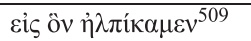 \\
\hline
\end{tabular}

Abb. 48: Intensivierende Aspektnuancierung im Resultativ.

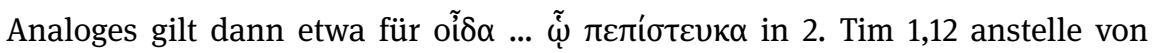

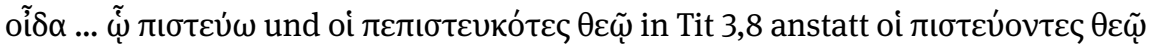
(vgl. Apg 2,44).

503 Das Deutsche scheint hier ganz ähnlich „Ich schreibe Dir dies“ und „Ich habe Dir dies geschrieben“ zuzulassen.

504 Vgl. CGCG 33.18 für eine Liste von im klassischen Griechisch betroffenen Verben. GGNT 197e

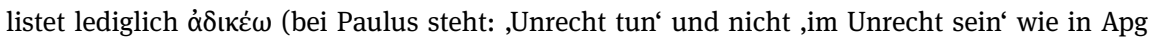
25,11) und vıкów (,Sieger sein` z. B. in Offb 2,7 statt ,siegen'). Es ist jedoch fraglich, ob hier nicht ebenfalls lexikalisch-semantische Erklärungsansätze genügen (so Stephen C. Carlson in noch unveröffentlichter Forschung).

505 Ps $144,15$.

506 1. Tim 5,5.

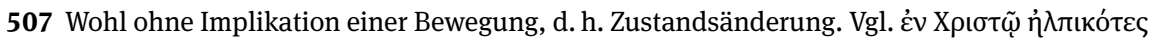
in 1. Kor 15,19.

508 2. Makk 2,18.

509 2. Kor 1,10. 


\subsubsection{Wahlmöglichkeiten bei transformativen Situationen}

Bei transformativen Verben hingegen sind letztlich nicht deckungsgleiche Situationen im Blick, da im Ind. Präs. im linearen Normalfall der Endpunkt der Situation als noch nicht erreicht dargestellt wird. Hier betrifft die im Hinblick auf den Aspekt mögliche „Wahl“ letztlich den Narrationstyp, ob also durch den Ind. Aor. in späterer Narration das Umschlagen der Zustände erzählt werden soll (wobei das Anhalten des Zustandes dann kontextuell vorausgesetzt sein kann) ${ }^{510}$ oder ob im Ind. Perf. in simultaner Narration ${ }^{511}$ der anhaltende Zustand festgehalten soll (wobei der Umschlag des Zustandes dann vorausgesetzt ist): Diese unterschiedlich ausfallende Entscheidung spiegelt sich etwa in Röm 6,4 („Christus wurde

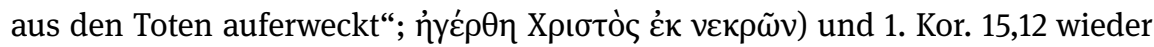

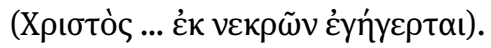

Innerhalb der simultanen Narration besteht nun im Hinblick auf Ind. Präs. und Ind. Perf. wiederum die Möglichkeit, dieselbe Situation mit unterschiedlicher Aspektnuance durch die Wahl verschiedener Lexeme im Ind. Präs. auf der einen und Ind. Perf. auf der anderen Seite zum Ausdruck zu bringen: So wird die Rede

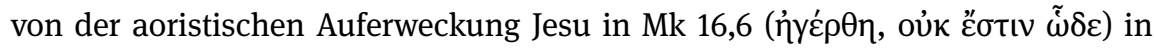
V. 11, also dem späteren Schluss, im Ind. Präs. vom Zustandsverb $\zeta \alpha \omega^{512}$ aufgegrif-

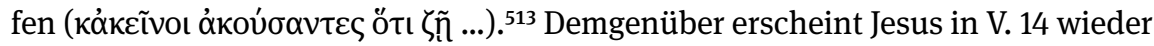

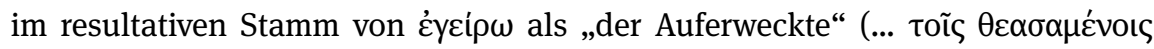

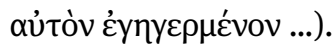

Es zeigt sich hier, dass die Wahl eines Lexems, welches im Ind. Perf. denselben Zustand ausdrücken kann, wie das Zustandsverb im Ind. Präs., in der Regel mit einer größeren Offenheit für intertextuelle Bezüge auf tatsächliche Erzählungen des Vorausgehenden einhergeht (vgl. die Binnenerzählung in Mk 16,6). Dementsprechend ist Aubrey zwar einerseits recht zu geben, dass in Jer 9,4 der Ind. Perf. von $\mu \alpha v \theta \alpha \dot{v} \omega$ das Resultat im Hinblick auf den Lernenden bezeichnet: „,kno-

510 Siehe oben, Abschnitt 3.4.2.3.

511 Aubrey, „Greek Perfect“ differenziert hier (im Hinblick auf 2. Tim 4,7 und Mt 7,28) zwischen narrativem Diskurs, welcher den Ind. Aor. bevorzuge, und direktem Diskurs. Es ist aber wohl eher zwischen späterer Narration (welche typisch für viele Erzählwerke ist) und simultaner Narration zu differenzieren. Vgl. hierzu die sehr dichte Einbettung in weitere Ereignisse in 2. Tim 4,6

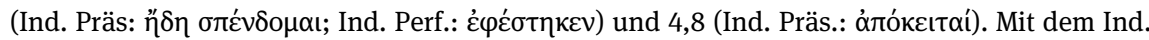

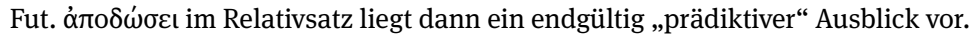

512 Es wurde bereits darauf hingewiesen (siehe oben, Abschnitt 3.4.2.3), dass der Gebrauch des Ind. Aor. für diese Zustandsverben keine aspektuelle Alternative bereitstellt, sondern vielmehr auf den Umschlagspunkt in der Vergangenheit fokussiert (und kontextuell ein Anhalten in die

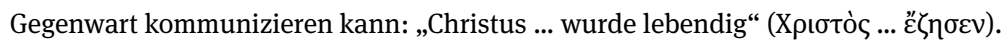

513 Vgl. Lk 24,5.23 und Röm 6,10. Teilweise kann natürlich auch eine andere inhaltliche Schwer-

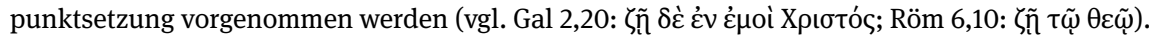


wing.“514 Die Frage „Wie kann es sein, dass er die Schrift so gut kennt, obwohl er

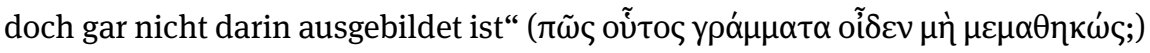
in Joh 7,15 zeigt demgegenüber aber auch, dass hier in der Resultativ-Form von $\mu \alpha v \theta \alpha \dot{v} \omega$ durchaus mehr mitschwingt, als nur das gegenwärtige „Wissen.“ Zwar ist Wissen in der Regel aktiv erworben (vgl. aber Gal 1,12!), doch liegt im Ind.

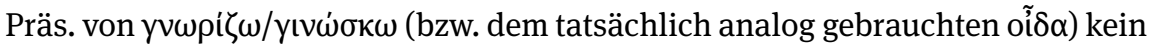
Fokus darauf. Der Resultativ bewirkt demgegenüber eine Reflexion der bisherigen Erzählung.

\subsubsection{Periphrastische Resultativ-Formen}

Eine zusätzlich mögliche Differenzierung in der Konzeptualisierung einer Situation besteht eventuell im Gebrauch periphrastischer Konjugation ${ }^{515}$ für Formen des Perfekt-Stamms. Levinsohn beschließt seine vergleichende Analyse wie folgt: „I conclude that it is indeed the case that copular perfects are less dynamic than their simple equivalents, with the copular form typically used for ongoing states (which result from completed events). "516 Dies beträfe in den Paulusbriefen etwa 2. Kor 4,3 und Eph 2,8 und 4,18.517

\subsection{Erzähltempora der „eingeschobenen“ Narration}

Die eingeschobene Narration weist keine eigenen Erzähltempora auf. Vielmehr ist für sie charakteristisch, dass sie abwechselnd auf Erzähltempora der späteren und dann wieder auf solche der simultanen Narration innerhalb desselben Textabschnittes zurückgreift. ${ }^{518}$ Diese Abfolge - gerade in Briefen und Tagebüchern - ist darin begründet, dass einmal die Perspektive des erzählten Ichs und

514 Aubrey, „Greek Perfect.“

515 Vgl. GGNT 200j.

516 Levinsohn, „Functions,“ 320. S. 324: Wegfallen der Kopula resultiert in „a loss of the dynamic contrast between simple and copular perfects.“

517 GGNT 203a beschränkt eine besondere Nuancierung auf den Gebrauch mit Ptz. der Kopula: In diesem Fall „kann das Andauern des erreichten Zustandes zusätzlich hervorgehoben werden."

518 Weinrich, Tempus würde hier eher davon sprechen, dass „Besprochenes in Erzähltes und Erzähltes in Besprochenes eingelassen ist“ (so S. 15 im Hinblick auf die Verschränkung der Tempora gerade im Alltag). Da hier an der absoluten Zeitbedeutung der griechischen Tempora festgehalten wird, ist das Phänomen aus der Interaktion der explizit temporal differenzierten simultanen und späteren Narration zu erklären. 
dann wieder die Perspektive des erzählenden Ichs maßgeblich für die Wahl der Orientierungszeit ist. 519

Charakteristisch für diesen Narrationstyp sind Überlagerungen in der Fokalisierung, die etwa zu Bewertungen des aus der Perspektive des erzählten Ichs geschilderten Geschehens führt, die diesem zum damaligen Zeitpunkt noch gar nicht vorlagen. So ist etwa in Gal 2,4 die Charakterisierung der Gegner (toù

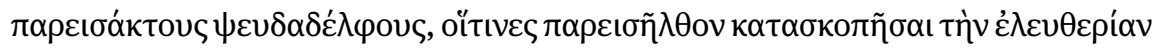
$\dot{\eta} \mu \tilde{\omega} v)$ vermutlich eine Darstellung, die Paulus so nach einer gewissen Reflexionszeit wählt. ${ }^{520}$ Sehr interessant ist auch das angebene Motiv für das Handeln des Petrus in Gal 2,12: „... weil er sich vor denen aus der Beschneidung fürch-

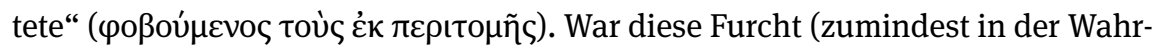
nehmung des Paulus) von außen sichtbar? Oder handelt es sich hierbei schlicht um einen bloß unterstellten mentalen Zustand? Oder aber fließt hier bereits die Erwiderung ein, die Petrus nach der in V. 15-21 erzählten Konfrontation als (in seinen Augen durchaus respektable) Begründung gegeben haben dürfte (unabhängig davon, ob er hier selbst von Furcht gesprochen haben mag oder Paulus seine Erklärung so auslegte)?

Anachronismen geschehen in der eingeschobenen Narration aber nicht nur im Hinblick auf die im Text gewählten Bewertungen. Auch die Bezüge auf textexterne Entitäten spiegeln zuweilen ein Verharren in einer der beiden Perspektiven wider, welches nicht mit dem im Text explizit angegebenen Perspektivenwechsel

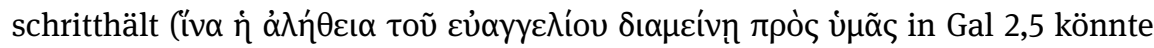
ein sehr markantes Beispiel hierfür sein; siehe unten, Kapitel 11, Abschnitt 6.2).

Genette bringt diese Spannung, welche auch die indikativischen Tempora betreffen kann, wie folgt zum Ausdruck:521

519 Im Rahmen einer Analyse, die von klassischen Kategorien der Rhetorik ausgeht, kann dieses Phänomen mit der Rede von der narratio partilis aufgegriffen werden (vgl. Wischmeyer, „Ich-Erzähler"). Allerdings ist aus narratologischer Perspektive doch daran festzuhalten, dass zwischen tatsächlichen Unterbrechungen der narrativen Vertextungen (siehe dazu etwa unten, Kapitel 8, Abschnitt 6) und einem Wechsel lediglich des Narrationstyps zu unterscheiden ist.

520 Genette, Erzählung, 141 scheint hier zu wenig mit unbewussten nachträglichen Rationalisierungen und Neubewertungen zu rechnen, bringt ansonsten aber die temporale Verschachtelung gut auf den Punkt: „Der Erzähler ist hier, und zwar zugleich, noch der Held und schon ein anderer: Die Ereignisse des Tages sind schon vergangen, und der point of view kann sich seitdem verändert haben; die Gefühle und Ansichten am Abend oder am folgenden Tag jedoch gehören völlig zur Gegenwart, und hier ist die Fokalisierung auf den Erzähler gleichzeitig eine Fokalisierung auf den Helden.“

521 Genette, Erzählung, 141. 


\begin{abstract}
Und schließlich bewirkt die sehr große Nähe zwischen Geschichte und Narration [d. h. zwischen Erzähltem und Erzählen] hier sehr oft, wenn ich so sagen darf, einen sehr subtilen Reibungseffekt zwischen der leichten Zeitverschiebung in der Erzählung von Ereignissen („heute ist mir dies und das passiert“) und der strikten Gleichzeitigkeit in der Mitteilung der Gedanken und Gefühle („heute abend denke ich so und so darüber“). Das Tagebuch und der vertrauliche Brief verknüpfen ständig das, was man in der Rundfunksprache Direktübertragung und Übertragung zu einem späteren Zeitpunkt [le direct et le différé] nennt, eine Art inneren Monolog mit einem nachträglichen Bericht.
\end{abstract}

Ein für das NT diskutiertes Phänomen in der Verwendung des Ind. Perf. könnte als Resultat dieser „Reibung“ verstanden werden. Wie oben (Abschnitt 3.3.2.3) erwähnt, erscheint der Ind. Perf. manchmal, wo man eigentlich das Plsqpf. erwarten würde, also für Resultatives, das vom Erzählzusammenhang eigentlich zu einem bestimmten Zeitpunkt in der Vergangenheit geltend ausgesagt sein sollte. Es stellt sich dann die Frage, weshalb ein Tempus gewählt ist, das im Normalfall Gegenwartsbezug aufweist.

So sticht etwa der oben bereits angeführte Vers 2. Kor 11,25 in dieser Hinsicht hervor: Auf die Schilderung der einmaligen Steinigung und der je dreimaligen Erfahrung der Auspeitschung und des Schiffbruchs im Aorist folgt im Perfekt die Aussage: „einen Tag und eine Nacht habe ich in Seenot zugebracht“

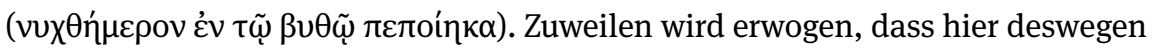
ein Tempus mit Gegenwartsbezug gewählt ist, weil die „bleibenden Auswirkungen des Geschehens" betont werden sollen. ${ }^{222}$ Gegenüber der eigentlich in der späteren Narration zu erwartenden Wahl des Plsqpf. (oder einer entsprechenden Ipf.-Form) soll also in der Tat ausgesagt werden, dass das Geschehen in der Vergangenheit auch noch in der Gegenwart nachwirkt. In diesem Fall könnte man die Wahl des Perfekts also als Begleiterscheinung der für die eingeschobene Narration typische Verwischung des temporalen Standpunktes des erzählenden und erzählten Ichs betrachten.

Wallace ${ }^{523}$ differenziert nicht weiter zwischen ,aoristischem“ und „dramatischem/historischem “ Perfekt, was zu der Situation führt, dass eine Kategorie, die gerade über die anhaltende Wirkung der vergangenen Situation definiert wurde, plötzlich daran erkennbar werden soll, dass dieses Element fehlt: „The key to detecting a dramatic perfect is the absence of any notion of existing results. "524 Robertson kommentiert demgegenüber $\tau \varepsilon \theta \varepsilon \dot{\alpha} \mu \alpha \iota$ in Joh 1,32 mit den Worten: „John still has that vision.“525 $\mathrm{Zu}$ Offb 5,7 schreibt er sogar: „A striking instance of

522 Vgl. etwa NSS 1028.

523 Wallace, Grammar, 578 mit Zitat von Robertson, Grammar, 896.

524 Wallace, Grammar, 578. Hervorhebung im Original.

525 Robertson, Grammar, 897. 
it is seen in Rev. 5:7, $\varepsilon \iota^{\prime} \lambda \eta \varphi \varepsilon v$, where John sees Jesus with the book in his hand. It is dull to make $\varepsilon \iota^{\prime \prime} \lambda \eta \varphi \varepsilon v$ here = $\varepsilon \lambda \alpha \beta \varepsilon v . " 526$ Im Hinblick auf Paulus meint er unter

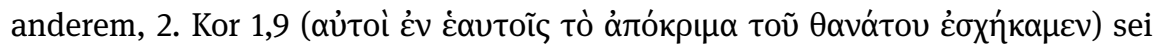
als „a dreadful memory to Paul“ zur Zeit des Briefschreibens noch gegenwärtig gewesen.

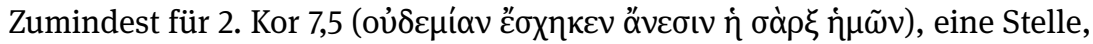
welche Robertson ebenfalls in diesem Zusammenhang anführt, und dementspre-

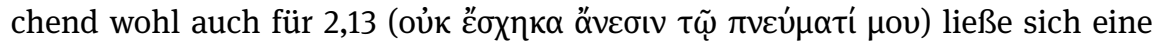
rhetorische Funktion dieser „vergegenwärtigten“ Sorge angeben, dass nämlich dadurch die klimaktische Tröstung Gottes durch die Ankunft des Titus (mit guter Nachricht aus Korinth) vorbereitet würde. Mit dem „Erleichterungsruf“ in 2,14 kann der Gebrauch in 2,13 allerdings nicht in Verbindung gebracht werden, da er ja in 7,5 wiederholt wird. Dass diese Spannung insgesamt im Text so aufrechterhalten werden soll ist fraglich, da die Versöhnung mit den Korinthern bereits vor 7,6 im Text vorausgesetzt (und den Lesern natürlich ohnehin bekannt) ist. ${ }^{527}$

Die Kategorie des „historischen Perfekts“ (Perfekt für Plusquamperfekt) ist unter anderem deswegen problematisch, da oft auch ein - sprachgeschichtlich im 1. Jh. nur eben strittiges - „aoristisches Perfekt“ (Perfekt für Aorist) den Gebrauch erklären könnte. Darüber hinaus ist aber vor dem Hintergrund der besonderen Konstellation zwischen erzählendem und erzähltem Ich in der eingeschobenen Narration auch zu überlegen, ob hier nicht oft tatsächlich ein - in konsequent späterer Narration sicherlich unerwarteter - uneingeschränkter Gegenwartsbezug vorliegt.

So ließe sich etwa 2. Kor 12,9 anführen, das Robertson ebenfalls unter dem historischen Perfekt anführt. Paulus erzählt in 2. Kor 12,8 im komplexiven Ind. Aor. von seinem dreifachen Beten zum Herrn bezüglich des „Stachels im Fleisch“

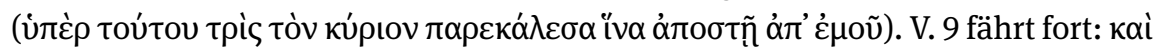

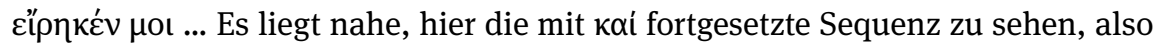

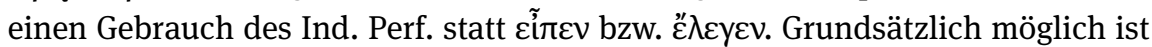
natürlich auch, dass Paulus die sich aus dieser sprachlichen Äußerung des Herrn ergebende Situation in der Vergangenheit analog zu cipńkєı ausdrücken wollte. ${ }^{528}$ Am einfachsten scheint jedoch die Option, dass hier schlicht ein Wechsel in die

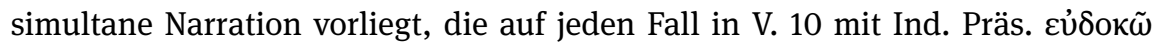
begegnet. Paulus würde dann den verbalen Akt des Herrn in der Vergangenheit übergehen und direkt zur gegenwärtigen Konsequenz übergehen. Auch in 2. Kor

526 Robertson, Grammar, 897.

527 Vgl. Heilig, Triumph, 157-158.

528 Das Missverständnis einer so ausgedrückten Vorvergangenheit (des Herrn Aussprach lag vor und dennoch betete Paulus) ist wohl aufgrund des Konnektors eher gering. 
12,17 hat Paulus vermutlich schlicht die Tatsache vor Augen, dass die in V. 18

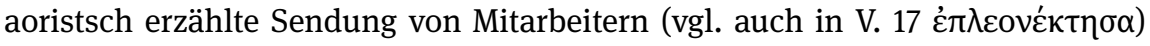
nach wie vor, in der Gegenwart des Briefschreibens, als seiner Verantwortung

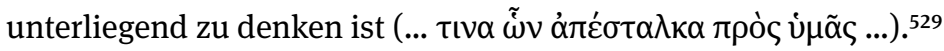

In 2. Kor 1,9, 2,13 und 7,5 scheint die Erklärung auf den ersten Blick wenig einleuchtend, da hier ja im Rahmen der Erzählung selbst das Ende des Zustan-

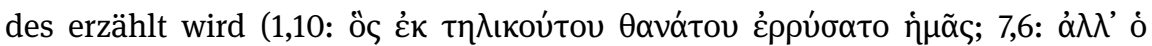

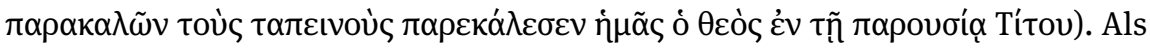
normaler Ind. Perf. kann die Form eigentlich nur gelten, wenn man von einem tatsächlich verhängten Todesurteil gegen Paulus ausgeht, denn auch im Fall einer anhaltenden Krankheit etc. wäre V. 10 ja als Beseitigung dieses Zustandes zu lesen (während ein Urteil fortbestehen kann, auch wenn die Flucht gelungen ist). ${ }^{530}$ Folgt man jedoch der Definition Crellins (s. o., Abschnitt 2.2.3.4) ist auch hier unabhängig von der genauen Referenz die Annahme eines Wechsels in die simultane Narration durchaus möglich. Paulus würde dann beispielsweise in 1,8 zunächst von den Beschwernissen in späterer Narration erzählen, um dann in 1,9 aus der Perspektive des erzählenden Ichs zu sprechen: Aufgrund dieser Vorkommnisse ist er nun jemand, der in der Vergangenheit schonmal am Leben verzweifelt war. Er ist in diesem Zustand der akuten Verzweiflung aber nun nicht mehr - wie die Leser in V. 10 erfahren, wo Paulus die Befreiung aus dieser Situ-

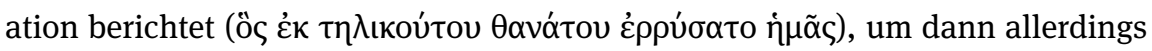

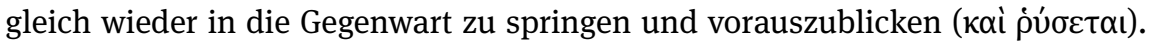

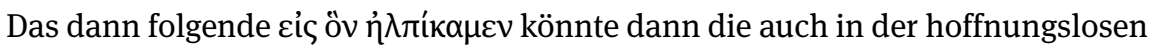
Situation V. 9 aufrechterhaltene Hoffnung meinen. Hält man das direkt folgende ǒtı für ursprünglich, wäre wohl eher (intensivierend) die gegenwärtige Hoffnung ausgedrückt, dass auch in der Zukunft des Briefschreibers Gott rettend eingreifen

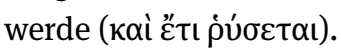

Sieht man in diesen Stellen kein „historisches Perfekt“ (also eine Verschiebung der Orientierungszeit in die erlebte Vergangenheit), ist das Auffällige also vielmehr ein überraschender Wechsel in die simultane Narration mit normaler Perfektbedeutung (im Rahmen der Definition von Crellin): Anstatt aus der Perspektive des erlebenden Ichs zu erzählen, was passierte, wird erzählt, was dies für das erzählende Ich bedeutet. Ein solcher Tempusgebrauch - ausgelöst durch den eingeschobenen Narrationstyp - wäre umso bemerkenswerter, als das Griechische

529 Vgl. Evans, „Ghost“ gerade zu ó̉ $\varepsilon \sigma \tau \alpha \lambda \kappa \alpha$ und dem angeblichen „epistolarischen Perfekt.“ Siehe hierzu oben, Abschnitt 3.2.2.

530 Gegen Schmeller, Brief I, 71. 
ansonsten ja durchaus die Tendenz aufweist, in der Vergangenheitsperspektive zu verharren, wo statt Ipf. auch Ind. Präs. möglich wäre (siehe oben, Abschnitt 3.4.1.2).

Allerdings gibt es durchaus Fälle, bei denen die Betrachtzeit - analog zum Aorist - zurück in eine Situationszeit verschoben scheint, welche vor der Sprechzeit $\mathrm{zu}$ einem Ende kommt. Besonders deutlich ist dies dort, wo nicht-finite Verbformen mit Bezug auf vergangene Ereignisse vom Ind. Perf. abhänig sind

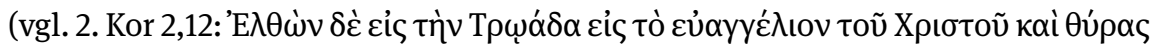

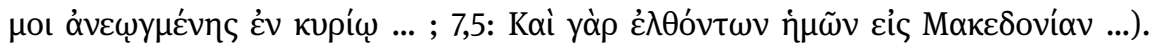
Ihren Zeitbezug erhalten diese Verbformen vom finiten Verb und ein aoristisches Verständnis des Ind. Perf. scheint daher kaum vermeidbar. Ähnliches gilt wohl auch für 1. Kor 15,4, wo die temporale Spezifizierung „am dritten Tag,“ welche deutlich vor dem „Heute“ liegt, mit dem Ind. Perf. verbunden ist. ${ }^{531}$ Anscheinend ist der beabsichtigte pragmatische Effekt der Einbeziehung der gegenwärtigen Situation in das Geschilderte so groß, dass Paulus hier die Grammatik missachtet und streng genommen eine Mischung aus Vergangenheitsbezug (temporale Bestimmung) und gegenwärtiger Auswirkung (Ind. Perf.) vornimmt, die den Ind. Perf. in Richtung Ind. Aor. verschiebt, ohne jedoch seine aspektuelle Besonderheit zu beseitigen, wie dies beim ,aoristischen Perfekt“ später der Fall sein sollte (vgl. Abschnitt 3.3.2.2).

\section{Diathese}

Damit ein Satz ein „Ereignis“ im hier geforderten Sinn zum Ausdruck bringen kann, muss das Verb, welches das Prädikat liefert, nicht notwendigerweise im Aktiv stehen. Auch passive Formen bringen Geschehnisse zum Ausdruck, wobei

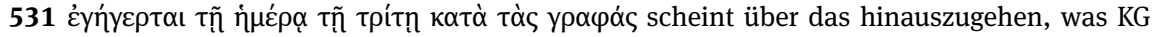
384 über die Verbindung des Ind. Perf. (und Ind. Präs.) mit „Zeitadverbien der Vergangenheit“ schreiben. Ind. Perf. mit „definite time adverbials“ ist wohl nur in nicht-literarischen Quellen belegt. Vgl. oben, Abschnitt 3.2.2 zu in P.Hib. 1.53. Siehe Crellin, Syntax and Semantics, 9-10.

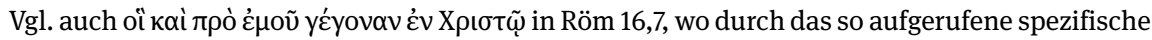
Ereignis vor Damaskus auch eine gewisse Spannung zum Gegenwartstempus entsteht. Interessanterweise wird die Ungewöhnlichkeit der temporalen Bestimmung mit dem Ind. Perf. in 1. Kor 15,4 selten wahrgenommen. GGNT 200e führt die Stelle lediglich zur Verdeutlichung des aspektuellen Unterschieds von Aorist und Resultativ an. Die NET merkt an, dass im Englischen Present Perfect und Temporalangabe grammatisch nicht zusammengehen - übersieht dabei jedoch, dass im Griechischen die Situation (aus etwas anderen Gründen) nicht unähnlich ist: „, he has been raised/is raised,' using a Greek tense that points to the present effect of the act of raising him. But in English idiom the temporal phrase ,on the third day ' requires a different translation of the verb.“ Auch im gesprochenen Englisch gibt es pragmatisch bedingte Abweichungen. 
im Griechischen das (im Fall des Aktivs im Subjekt enthaltene) Agens durch ن́ró + Genitiv optional mitangegeben werden kann. ${ }^{532}$ Eine gewisse Ausnahme stellt das sogenannte „tolerative“ Passiv dar. ${ }^{533}$ So stellt sich in Röm 1,20 die Frage, ob durch den Indikativ Präsens Passiv $\kappa \alpha \theta o \rho \alpha ́ \omega$ ein regelhaftes, tatsächlich stattfindendes Ereignisbündel erzählt wird („es wird gesehen“) oder im tolerativen Sinn lediglich die Möglichkeit dieses Geschehens betont wird (,es lässt sich erkennen“). ${ }^{534}$

Die „unmittelbare textfunktionale Relevanz der Genera Verbi“ aufgrund der „lineare[n] Ordnung der Argumentausdrücke“ im Text ${ }^{535}$ ist im Griechischen nur modifiziert anwendbar: Während im Deutschen die Wahl des Passivs die Vermeidung der expliziten Nennung des Agens ermöglicht, kann im Griechischen auch im Aktiv eine gewisse Defokussierung des Agens erfolgen, indem das Subjekt alleine durch die Personalendung des Verbs ausgedrückt wird. ${ }^{536}$ Soll das Agens jedoch gar nicht erwähnt werden, ist auch hier das Passiv die entsprechende Wahl, wobei dann zusätzlich das Patiens als Subjekt im Passivsatz entweder explizit erwähnt werden kann oder auch nicht. ${ }^{537}$

Wenn das Agens im Kontext genannt ist, stellt die Wahl des Passivs im Deutschen entsprechend einen Fall impliziter Themakontinuität dar, ,bei der der (noch) thematische Gegenstand als weniger relevant gegenüber neuer rhematischer Information in den Hintergrund gerückt, defokussiert wird. "538 Im Griechischen Aktiv kann hier schlicht, das bei expliziter Nennung direkt auf das Verb folgende thematische Element unerwähnt bleiben. ${ }^{539}$

Relevanter sind daher Fälle, in welchen das Agens nicht im weiteren Kontext erwähnt wird. Hier kann entweder auf Weltwissen aufgebaut (so wohl beim passivum divinum $)^{540}$ und unter Umständen eine Generalisierung vorgenommen werden ${ }^{541}$ - oder aber es liegt eine tatsächliche „Anonymisierung“(vgl. etwa 1. Kor 11,23) vor, die aber selbst wiederum sehr unterschiedliche diskursive Funktionen

532 GGNT 191a. Auch andere Präpositionen sind vereinzelt möglich. Siehe etwa 2. Kor 1,11 für

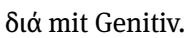

533 Vgl. GGNT 191h.

534 Vgl. NSS 902.

535 Zifonun, „Textkonstitutive Funktionen,“ 316.

536 GGNT 255d.

537 Vgl. GGNT 255a.

538 Zifonun, „Textkonstitutive Funktionen,“ 316.

539 CGCG 60.26. Siehe zur Thema-Rhema-Gliederung grundsätzlich oben, Kapitel 4, Abschnitt 8.1.3.

540 Vgl. zu diesem GGNT 296b. Das Gesagte gilt aber wohl auch für andersartige Einflüsse: vgl. Röm 15,22 und 1. Kor 12,2.

541 Siehe auch 255f zu Röm 10,10. 
erfüllen kann. ${ }^{542}$ Narratologisch kann grundsätzlich eine Korrelierung mit geringerer Anschaulichkeit (vgl. oben, Kapitel 3, Abschnitt 4.3) der Erzählung erwogen werden.

Die Reduktion des Informationsgehalts muss jedoch nicht notwendigerweise mit einer Abstufung in der Informationsstruktur verbunden sein: Wo die Nennung eines Agens aufgrund der konventionalisierten Konzeptualisierung des Geschehens standardmäßig zu erwarten wäre, kann die auffälligere Agens-freie Darstellung auch Aufmerksamkeit auf sich ziehen und ein Ereignis (bzw. seine Verursachung) ${ }^{543}$ als für das Erzählte besonders relevant markieren.

Grundsätzlich ist bei der Berücksichtigung der Diathese in der Analyse griechischer Texte zu beachten, dass anders als etwa im Englischen das Passiv keine stets vom Aktiv abgeleitete Form darstellt. ${ }^{544}$ Vielmehr stehen sich hier nicht-abgeleitete aktive und mediale Formen gegenüber (die daher auch ohne Entsprechung auf der anderen Seite existieren können), wobei die Formen im Aorist mit - $(\theta) \eta$ - innerhalb der Domäne des Mediums (an der Seite der sigmatischen medialen Formen) einen bestimmten semantischen Bereich abdecken ${ }^{545}$ und die Markierung von Passivität nur eine von mehreren Funktionen ist. ${ }^{546}$ (Dieser Vorbehalt muss in der hier weitestgehend beibehaltenen Rede von „Passiv-Formen“ mitgedacht werden.) Entsprechend wäre für die Texte des NT im Hinblick auf die Diathese auch vor allem die jeweilige Konzeptualisierung der Situation - im Hinblick auf Energie-Quelle und -Endpunkt ${ }^{547}$ und Fokussierung der Aufmerksamkeit auf Beteiligte - zu beachten, welche durch die im Aorist-Stamm vorliegenden unterschiedlichen medialen Formen erreicht werden kann. ${ }^{548}$ Eine sehr hilfreiche Übersicht über die unter diesen Gesichtspunkten zu differenzierenden Situationstypen bietet nun Rachel Aubrey. ${ }^{549}$

542 Vgl. Zifonun, „Textkonstitutive Funktionen,“ 326. Vor diesem Hintergrund ist die Wahl des Aktivs in 1. Kor 2,8 besonders relevant: Paulus hätte hier eine entsprechend vage Option zur Verfügung gestanden, wenn er aus Furcht vor Konsequenzen offene Kritik an römischen Offiziellen gescheut hätte. Vgl. Heilig, Hidden Criticism, 127-129.

543 Sehr fraglich erscheint daher die These, dass im Passiv grundsätzlich das Subjekt hervorgehoben werden soll (Yoon, „Prominence,“ 13).

544 Vgl. Aubrey, „Motivated Categories,“ 582-584. Zum Deutschen siehe S. 585.

545 Siehe für eine gute Übersicht Aubrey, „Motivated Categories,“ 612-620.

546 Was klassischerweise mit der Rede von „aktiven Passivformen“ eingefangen wird. Dies ist aber vor dem genannten Hintergrund aber nicht unproblematisch. Siehe Aubrey, „Motivated Categories,“ 619. Auch das „tolerative Passiv“ kann wohl letztlich als eine Erweiterung der „spontanen Prozesse“ verstanden werden.

$547 \mathrm{Zu}$ Transitivität als prototypischer Kategorie siehe auch oben, Abschnitt 2.2.3.

548 Vgl. Aubrey, „Motivated Categories,“ 617.

549 Aubrey, „Motivated Categories,“585-612. 


\section{Modus}

\subsection{Allgemeines zum Indikativ}

Innerhalb der (affirmativen) Behauptungssätze lässt sich anhand des verwendeten Modus weiter differenzieren, ob der Sprecher/Schreiber das Behauptete als wirklich, ${ }^{550}$ irreal oder potenzial darstellt. ${ }^{551}$ Der für Ersteres gebräuchliche Indikativ ${ }^{552}$ stellt somit den Standard-Modus für das Erzählen von Ereignissen im oben definierten Sinne dar. So schreibt schon Genette: 553

Die Erzählung hat ja nicht die Funktion, einen Befehl zu erteilen, einen Wunsch zu formulieren, eine Bedingung anzugeben usw., sondern bloß die, eine Geschichte zu erzählen, d. h. über (wirkliche oder fiktive) Tatsachen zu „berichten,“ so dass ihr einziger, jedenfalls ihr charakteristischer Modus streng genommen nur der Indikativ sein kann. Damit wäre auch schon alles zu diesem Thema gesagt, es sei denn, man wollte einen übertrieben dehnbaren Gebrauch von der linguistischen Metapher machen.

Entsprechend werden Verbformen in den Modi des Konjunktivs, Optativs und Imperativs generell hier nicht als Erzählungen konstituierende Elemente gewertet, da das durch das Verb zum Ausdruck gebrachte Geschehen „als etwas Gewolltes oder subjektiv Erwartetes, “ „als erfüllbare[r] Wunsch ... oder als nur gedachte Möglichkeit“ bzw. als an die 2. oder 3. Person ergehende „Aufforderung“ betrachtet wird. ${ }^{554}$

550 Grundsätzlich ist der „Wirklichkeitsbezug“ immer im Hinblick auf die jeweilige erzählte Welt zu verstehen. Diese kann die reale Welt des Autors sein, muss es aber nicht.

551 GGNT 267. Ebenso wie Aspektualität auch jenseits des Aspekts kommuniziert werden kann (vgl. oben, Abschnitt 2), ist der grammatische Modus lediglich ein Teilaspekt der größeren Kategorie der Modalität. Siehe grundsätzlich oben, Kapitel 4, Abschnitt 5.1.2 zur „epistemischen Minimaleinheit.“ Vgl. nun auch AGG 207d für eine sehr ausführliche Gegenüberstellung von griechischer und englischer Umsetzung der Modalisierung. Insbesondere Kapitel 12 (v. a. Abschnitt 7.4 zur lexikalischen Abschwächung) und Kapitel 14 (z. B. Abschnitt 3) liefern hier zahlreiche Einzelbeobachtungen zu Sprechhandlungen, die nicht für Erzählungen konstituierend sind, ohne dass dies am Modus erkannt werden könnte.

552 Dies schließt natürlich nicht aus, dass der Indikativ in bestimmten Fällen auch auf Nichtwirkliches Bezug nehmen kann (GGNT 209a). Zu irrealen Konditionalsätzen siehe etwa unten, Kapitel 12, Abschnitt 8.3. Ebenso kann der Verbinhalt in abhängigen Behauptungssätzen natürlich durch die Semantik der übergeordneten Konstruktion ganz unterschiedlich modalisiert werden (siehe unten, Kapitel 14, Abschnitt 3.3 mit zusätzlichem Zukunftsbezug; vgl. auch Kapitel 12, Abschnitt 7.4 für andere Formen der lexikalischen Abschwächung mit Vergangenheitsbezug).

553 Genette, Erzählung, 103.

554 GGNT 64d. 


\subsection{Beispiel konsekutive Konnexionen}

Beispielsweise wird in Gal 2,13 nach einem indikativischem Hauptsatz mit $̋ \sigma \tau \varepsilon$ und Indikativ eine konsekutive Konnexion formuliert, wobei das in der RESULTAT-Proposition im Indikativ ausgedrückte Geschehen als tatsächlich realisiert zu verstehen ist: „Und es heuchelten mit ihm [auch] die anderen Juden, sodass

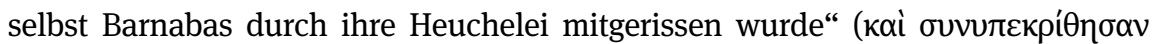

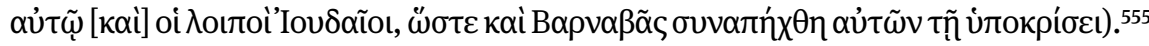

Im Kontrast dazu, stellt Gal 5,16 keine Erzählung im hier verfolgten Sinne dar: Das RESULTAT ist hier mit kaí consecutivum angefügt: „... und dann werdet

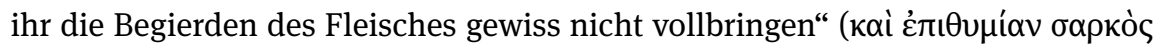
oủ $\mu$ ஸ่ $\tau \varepsilon \lambda \varepsilon ́ \sigma \eta \tau \varepsilon)$. Schon hier zeigt sich, dass die Konnexion keine Erzählung darstellt, da Zukunftsbezug vorliegt und noch dazu ein Ereignis vehement verneint wird. Damit stimmt überein, dass die Geschehens-Proposition hier im Modus des

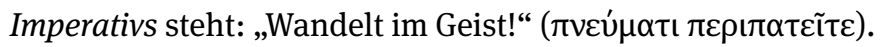

In 1. Kor 13,2 muss das Urteil ebenso ausfallen, denn hier steht das Geschehen im Konjunktiv, da es Teil eines prospektiven Konditionalsatzes ist, der den Sachverhalt eben nicht aussagt, sondern nur als mögliche Bedingung erwägt „Wenn ich allen Glauben habe ...“ ( die sich mit $̋ \sigma \tau \varepsilon$ anschließende RESULTAT-Proposition auch keine indikativische Verbform, sondern den Infinitiv für die lediglich gedachte Folge (, „... sodass

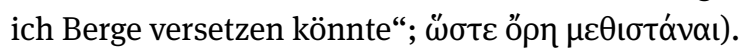

\subsection{Konjunktiv im Nebensatz}

Wo eine finite Verbform vorliegt, 556 liefert der Modus also durchaus ein wichtiges Kriterium, um der Frage nach Narrativität auf der Grammatik-Seite des Textes nachzugehen. Es muss jedoch an dieser Stelle auch eine Einschränkung vorgenommen werden: auch konjunktivische Verbformen können im Einzelfall Erzähl-Ereignisse im hier verfolgten Sinn ausdrücken. Weitaus am relevantesten sind hierbei die konjunktivischen Verbformen in Temporalsätzen, ${ }^{557}$ die nicht nur vorausschauend sind, sondern sich auch auf (besonders wiederholt) Vor- und Gleichzeitiges in der Gegenwart beziehen können und in manchen Fällen Ver-

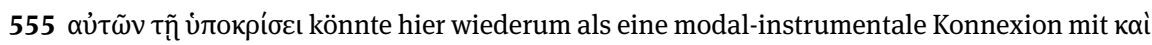

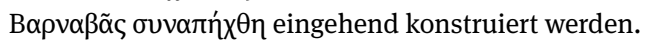

556 Siehe unten, Abschnitt 7 für infinite Verbformen.

557 GGNT 210i. 


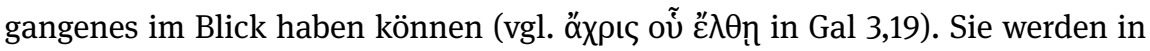
Kapitel 7 im Detail besprochen.

Konsekutives Ǐv (mit Konjunktiv) für als tatsächlich folgend Dargestelltes ist wohl (trotz aller Diskussionen) auch im NT belegt. ${ }^{558}$ Offb 13,13 „Und [das Tier] tat große Zeichen, __ es auch machte, dass Feuer vom Himmel auf die Erde vor die

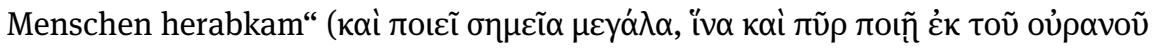

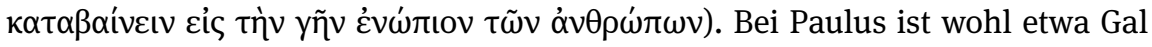
5,17 so zu verstehen: „[Geist und Fleisch] stehen einander gegenüber, __ ihr

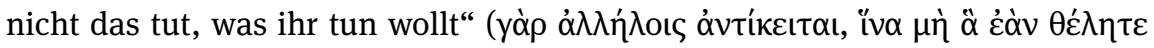

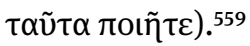

Besonders Caragounis hat sich außerdem dafür stark gemacht, unter Berücksichtigung der diachronen Entwicklung von ǐv (bzw. va), auch von einer Funktion als kausalem Konnektor auszugehen. ${ }^{560}$ Haubeck/von Siebenthal merken im Hinblick auf Mk 4,12 an, dass ein solches Verständnis (vgl. etwa NGÜ) „sprach1[ich] zwar z. T. nicht unmögl[ich]“ sei, aber doch „forciert“ erscheine. ${ }^{561}$ Für die hier verfolgte Fragestellung ist die Diskussion insofern relevant, als mit Röm 5,20 eine theologisch zentrale Stelle bei Paulus betroffen ist, die zudem unter narrativen Gesichtspunkten von Interesse ist: „Das Gesetz kam dazu, damit die Sünde

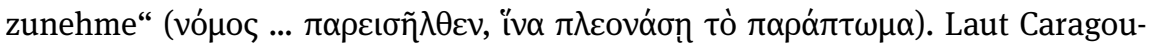
nis ist das zeitliche Verhältnis umgekehrt: Das Gesetz wurde gegeben, „weil die Sünde zugenommen hatte. “562 Caragounis verweist auf Gal 3,19: „Warum nun das Gesetz? Der Übertretungen wegen wurde es hinzugefügt“ (Tí oưv ò vó $\mu$ os; $\tau \tilde{\omega} v$

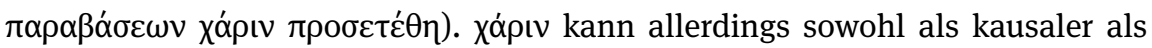
auch als finaler Konnektor auftreten, sodass Caragounis' folgende Beobachtung nicht zwingend ist: „According to this text the law becomes necessary because of the proliferation of sin. " ${ }^{653}$ Allerdings ist zumindest auch fraglich, ob umgekehrt

\section{GGNT 210i.}

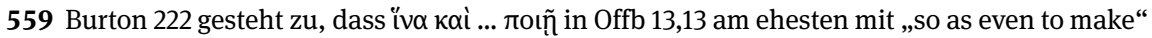
zu übersetzen ist. Seine Erklärung zu Gal 5,17 kann so verstanden werden, dass er das Fleisch als Fokalisierungsinstanz wählt ( Gal 5,17 ,is also best explained as expressing the purpose of the hostility of the flesh and the Spirit, viewed, so far as the iv clause is concerned, as a hostility of the flesh to the Spirit“). Ähnlich könnte man den „divine purpose“ verstehen, den Robertson, Grammar, 998 diskutiert, aber zumindest in Röm 11,11 unmöglich als Erklärung gültig ansieht.

560 Caragounis, Development, 221-224.

561 NSS 236.

562 Caragounis, Development, 223: „the law was given because sin had abounded.“

563 Caragounis, Development, 223. Erwogen werden kann, ob das Fragepronomen einen Hin-

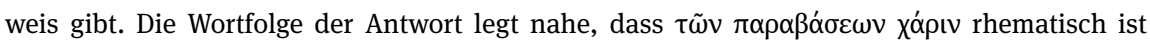
(vgl. CGCG 60.23). Die Frage hat also einen relativ engen Fokus: „Warum wurde also das Gesetz hinzugefügt“? Antwort: „Der Übertretungen wegen.“ 
die Interpretation der Galater-Passage mit Verweis auf die Römer-Stelle so einfach aufgelöst werden kann. ${ }^{564}$

Der Relativsatz mit konditionalem Nebensinn in Gal 5,17 (å $\pi$ กоп̃ $\tau \varepsilon)$ verweist zudem auf einen weiteren Gebrauch des prospektiven Konjunktivs. ${ }^{55}$ Die generelle Perspektive eignet sich jedoch kaum für Erzählungen mit Blick auf die Vergangenheit. ${ }^{566}$

\subsection{Modus und Differenzierung zwischen Vertextungsstrategien und Textsorten}

Die Analyse der Grammatik-Seite des Textes liefert nicht nur Kategorien zur Identifizierung narrativer Vertextungen. Vielmehr liefert auch die grammatische Konfiguration der den Propositionen zugrundeliegenden Sätze ein Unterscheidungskriterium zwischen (a) verschiedenen Vertextungsmustern und damit oft auch (b) zwischen verschiedenen Textsorten. Der Modus ist hier eines der grundlegendsten Eigenschaften der Grammatik-Seite des Textes, die es zu berücksichtigen gilt. ${ }^{567}$

So kann beispielsweise das von Gansel und Jürgens im Anschluss an Brinker vorgestellte deskriptive Vertextungsmuster („DESKR II“) $)^{568}$ auch anhand dieses Kriteriums von der Narration unterschieden werden: Das besagte Vertextungsmuster bezieht sich ,auf die Beschreibung eines Vorganges, eines Prozesses, der

564 Unter anderem an dieser Stelle zeigt die die Differenz zwischen den „Neuen Paulusperspektiven“ von Wright und Dunn: Für Letzteren ist das Gesetz gegeben, um als „boundary marker“ Israel vom sündigenden Heidentum abzuhalten, während Wright hier eine „klassischere“ Funktion sehen kann, das Sündigen aber nicht auf das Individuum, sondern auf das Volk als Kollektiv bezieht. Entsprechend wird der Status des unter dem „Fluch des Gesetzes“-Sein (Gal 3,10) ganz unterschiedlich aufgelöst. Vgl. Heilig, „New Perspective,“ 461-463.

565 GGNT 210j.

566 Siehe aber Offb 13,15: „Und es wurde ihm gegeben, dem Bild des Tieres Leben einzuhau-

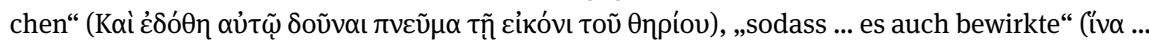

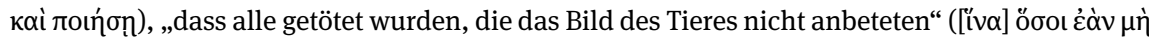

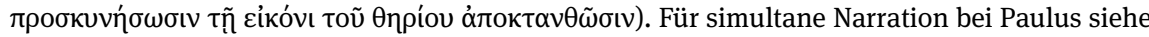

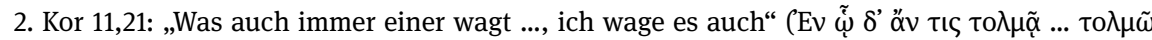
кỏy $(\dot{)})$.

567 Braun, Studien, 84 bemängelt, dass zwar in einigen Arbeiten deutlich herausgestellt werde, dass „drei der grammatischen Kategorien des Verbs, Tempus, Modus und Genus verbi, bei der Herstellung textueller Kohärenz bzw. ,Kohäsion“ eine wichtige Rolle spielen,“ dass „dieser Aspekt [gleichwohl] in gängigen Einführungen ... kaum bzw. nur in sehr kurzer und oberflächlicher Weise Beachtung“ finde.

568 Siehe Gansel und Jürgens, Textlinguistik, 155 und vgl. Brinker, Textanalyse [4. Aufl.], 63. 
sich generell durch Wiederholbarkeit auszeichnet."569 Beispielsweise macht die Textsorte „Bedienungsanleitung“ typischerweise von dieser Vertextungsstrategie Gebrauch: ${ }^{570}$

Schalten Sie das Radio durch Drücken von RADIO ON/OFF/TIMER OFF ein. Drücken Sie BAND zur Wahl des gewünschten Wellenbereichs. Drücken Sie die gewünschte Stationstaste. Zunächst erscheinen Wellenbereich, Frequenz, Einschalt-Anzeige und Stationsnummer im Display. Fünf Sekunden später erscheint die aktuelle Uhrzeit, die Stationsnummer bleibt weiter angezeigt.

Es handelt sich hierbei um ein deutlich chronologisch fokussiertes Propositionalgefüge (Sequenz: „Zunächst ... Fünf Sekunden später ...“; gleichzeitig: „weiter“). Dennoch handelt es sich deutlich nicht um eine narrative Vertextung, ${ }^{571}$ wie die formale Gestaltung zeigt. Die präsentischen Verbformen ließen sich zwar mit simultaner Narration vereinbaren, bereits die ersten beiden auf die Überschrift ${ }^{572}$ folgenden Sätze markieren durch den Modus jedoch bereits die deskriptive Vertextungsstrategie.

\section{Satzart}

Es wurde bereits darauf hingewiesen, dass die Frage „Hängt der Apfel am Baum?,“ die Aufforderung „Der Apfel hänge am Baum!“ und die Aussage „Der Apfel hängt am Baum“ einen gemeinsamen propositionalen Gehalt aufweisen. ${ }^{573}$ Es handelt sich dabei jedoch um verschiedene Satzarten, womit „typische Kombinationen von Äußerungsart und Satzform“ gemeint sind. ${ }^{574}$ Mit dem erstgenannten Aspekt ist dasjenige gemeint, was der Sprecher durch seinen Akt der Äußerung bewirken

569 Gansel und Jürgens, Textlinguistik, 153.

570 Das Beispiel wird bei Gansel und Jürgens, Textlinguistik, 154 angeführt.

571 Die Beurteilung des Musters DESKR III ist weniger eindeutig. Im Unterschied zur Narration stelle hier „der erste Satz des Textes den Leitsatz, die Neuigkeit“ heraus (Gansel und Jürgens, Textlinguistik, 155). Man könnte diesen Satz im Einzelfall jedoch auch als meta-narrativen Kommentar des Erzählers oder als Prolepse - und das Vertextungsmuster daher als eine komplexere Variante des narrativen Grundtyps - begreifen.

572 „Abrufen eines gespeicherten Senders.“ Bereits dieses Satzäquivalent (vgl. Duden 1404) liefert einen Hinweis auf die Textsorte, da man z. B. bei einer Überschrift eines Zeitungsartikels eher ein Aktionsnomen als den Infinitiv erwarten würde, z. B.: „Abberufung von Diplomat Schneider aus Beirut“ (vgl. Duden 391).

573 Vgl. GGNT 312a.

574 Duden 1388. 
möchte, also die kommunikative Funktion der Äußerung. Nur begrenzt entsprechen syntaktische Satzmuster dieser Einteilung. ${ }^{575}$

Grundsätzlich lässt sich anhand der kommunikativen Funktion von Sätzen zwischen Behauptungssätzen, Begehrsätzen und Fragesätzen unterscheiden. ${ }^{576}$ Damit ein Satz eine Ereignis-Repräsentation für eine Erzählung beisteuern kann, ist es eine notwendige Bedingung, dass es sich dabei nicht um einen Frage- oder Aufforderungssatz handelt. ${ }^{577}$ In der Syntax schlägt sich diese Funktion zumindest teilweise nieder (vgl. zum Modus in Aufforderungssätzen oben, Abschnitt 5, und zu Fragepartikeln unten, Kapitel 14, Abschnitt 2.1). Zudem muss es sich um einen affirmativen Satz handeln, es darf also das Prädikat des Satzes nicht negiert sein..$^{578}$

\section{Satzäquivalente und Textpragmatik}

\subsection{Hinführung}

Die im letzten Abschnitt kurz gestreiften Konsekutivsätze, eignen sich, um noch ein weiteres Phänomen zu besprechen: den Gebrauch von infiniten Satzäquivalenten in Erzählungen. Grundsätzlich gilt: Als Verbalnomina bringen Infinitive und Partizipien (neben Diathese und Aspekt) nur den Verbinhalt zum Ausdruck aber weder Modus noch (außerhalb des Futurs) Zeitbedeutung. ${ }^{579}$ Anders als indikativische Verbformen können sie in Isolation folglich nicht erzählen. ${ }^{580}$

575 Siehe auch CGCG 38.1-3 für das Griechische. Vgl. Duden 1389 für das Deutsche. 576 GGNT 266a.

577 Der Umkehrschluss gilt nicht: Behauptungssätze können z. B. Sachverhalte auch explizit als irreal darstellen. Bei abhängigen Behauptungssätzen spielt zudem die Semantik des übergeordneten Hauptsatzes eine große Rolle.

$578 \mathrm{Zu}$ Ereignisbezügen in solchen Sätzen siehe unten, Kapitel 10 und 12.

579 Zum Infinitiv: GGNT 213a und 214. Zum Partizip: GGNT 227a.

580 Selten (besonders im Zweiten Korintherbrief) ist bei Paulus das Partizip auch ohne finite Bezugsform verwendet (beziehungsweise ist der Bezug nicht immer klar rekonstruierbar; vgl. für die verschiedenen Grade Robertson, Grammar, 439-440). In diesen Fällen wäre also eigentlich eine finite Form zu erwarten und es liegt keine syntaktische Unterordnung vor (vgl. GGNT 231j). Siehe etwa für ein Beispiel in narrativem Kontext 2. Kor 7,5: „Und als wir nach Mazedonien kamen, hatten wir keine Ruhe in unserem Fleisch, sondern (wir waren) in allem bedrängt“

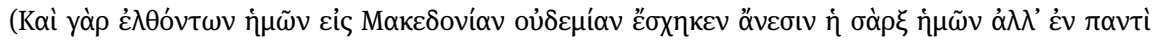
$\theta \lambda ı \beta o ́ p \varepsilon v o t)$. 


\subsection{Kontextuell gesicherter Vergangenheits- und Wirklichkeitsbezug}

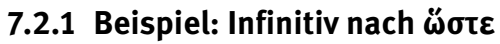

Dennoch kann der Infinitiv in bestimmten Kontexten für tatsächlich realisierte Ereignisse stehen, wie sich anhand der angesprochenen Konsekutivsätze zeigen lässt. Im klassischen Griechisch signalisiert der Gebrauch des Infinitivs im Konsekutivsatz, dass nicht spezifiziert ist, ob das Resultat tatsächlich geschieht oder geschehen ist, „merely that the action in the matrix clause is of a kind which enables or favours the bringing about of the result." 581

In der Koine ist die Differenzierung zwischen finiten (in der Regel indikativischen) und infiniten Verbformen jedoch so nicht mehr aufrechterhalten. Der

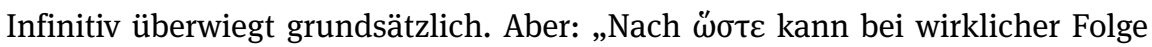
auch der Indikativ stehen."582 Der klassisch zu erwartende Indikativ steht außer in Gal 2,13 nur noch in Joh 3,16.583 So wird man beispielsweise auch 2. Kor 1,8 als Erzählung ${ }^{584}$ ansprechen dürfen: „Wir wurden über die Maßen, über (unsere)

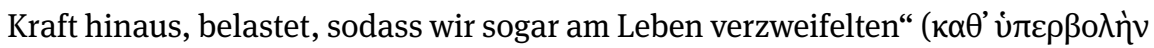

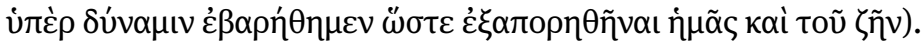

\subsubsection{Beispiel: innerliche Abhängigkeit}

Infinitive und Partizipien können auch in anderen Kontexten ohne erkennbaren Bedeutungsunterschied $\mathrm{zu}$ alternativen indikativischen Konstruktionen verwendet werden. Besonders deutlich ist dies in Fällen, in denen ,innerliche Abhängigkeit“ vorliegt, wenn also „die jeweilige Aussage oder Frage als Äußerung oder Gedanke der Subjektgröße der übergeordneten Konstruktion auftritt, die redet bzw. sinnlich oder geistig wahrnimmt.“585

Hier kann eine scheinbare Zeitbedeutung der infiniten Konstruktion ausgemacht werden, da das Gemeinte ohne erkennbaren Bedeutungsunterschied auch durch indikativische Vergangenheitstempora ausgedrückt werden kann. ${ }^{586}$ Zum hier durch die übergeordnete Konstruktion implizierten Vergangenheitsbezug kommt zudem dann indirekt Wirklichkeitsbezug hinzu, wenn das Verb der übergeordneten Konstruktion dies von seiner Semantik her impliziert. So ist

581 CGCG 46.7.

582 GGNT 221c.

583 Caragounis, Development, 183.

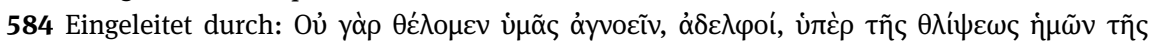

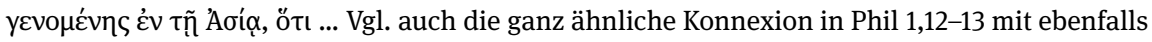
vergleichbarer Einleitung. Siehe dazu auch unten, Kapitel 8, Abschnitt 5.2.2.2.

585 GGNT 205.

586 GGNT 205. 


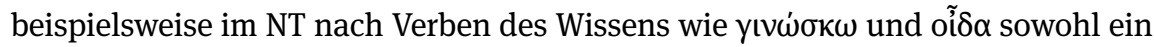
indikativischer ötı-Nebensatz möglich als auch die Konstruktion mit AcP oder AcI möglich. ${ }^{587}$ Die Situation ist folglich anders als im Deutschen, wo die innerliche Abhängigkeit durch den Konjunktiv I markiert werden kann, wodurch „der Sprecher seine Äußerung als referiert markiert, “588 sodass der uneingeschränkte Wirklichkeitsbezug der Aussage nicht mehr gegeben ist. Im NT ist eine solche Differenzierung grammatisch nur sehr bedingt möglich, da der klassisch gebräuchliche oblique Optativ bei indirekten Äußerungen im NT nur durch Lukas belegt ist. ${ }^{589}$

Es liegen hier also alternative Ausdrucksweisen einer Einleitung-INHALTKonnexion vor, die ein Erzähler verwenden kann, um seine Erzählung einzuleiten. So verwendet Paulus beispielsweise in 2. Kor 12,2 und 12,3-4, wie Abb. 49 zeigt, erst eine AcP-Konstruktion und dann einen ǒ $\tau$-Satz, um dasselbe Geschehen zu erzählen (im ötı-Satz jedoch ausführlicher).

\begin{tabular}{|c|c|}
\hline 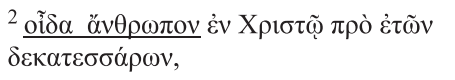 & 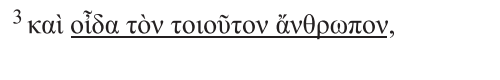 \\
\hline 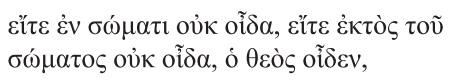 & 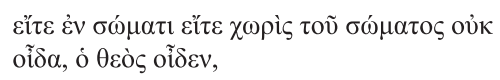 \\
\hline 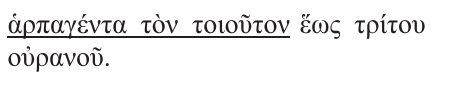 & 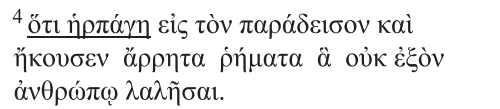 \\
\hline
\end{tabular}

Abb. 49: 2. Kor 12,2 und 2. Kor 12,3-4 im Vergleich.

Der Spezialfall der innerlichen Abhängigkeit zeigt einerseits, dass auch infinite Verbformen ohne erkennbaren Bedeutungsunterschied als Ersatz für indikativische Sätze gebraucht werden können. Zugleich wird jedoch auch die starke kontextuelle Abhängigkeit des kommunizierten Wirklichkeitsbezugs deutlich: In Lk 24,23 wird etwa von den das Grab besucht habenden Frauen gesagt: „Sie kamen und sagten, dass sie auch eine Erscheinung von Engeln gesehen hätten“

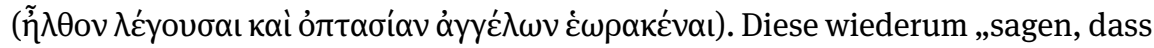

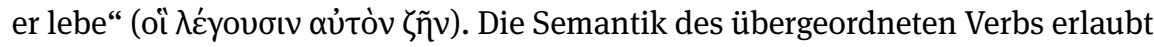
hier, dass die Wiedergabe des Berichs erfolgen kann, ohne dass die Sprecher sich auf die Tatsächlichkeit der Erscheinung festlegen müssten (vgl. Lk 24,11: „es

587 GGNT 233c.

588 Duden 1849. Vgl. Fußnote 53 bei GGNT 205.

589 GGNT 211fg. 


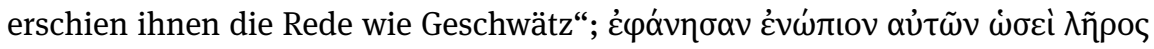
$\tau \grave{\alpha} \dot{\rho} \eta \dot{\mu} \tau \alpha \tau \alpha \tilde{v} \tau \alpha) .{ }^{590}$

\subsubsection{Adverbialer Gebrauch des Partizips}

Die zitierte Passage verweist durch das die erste Ebene der indirekten Rede ein-

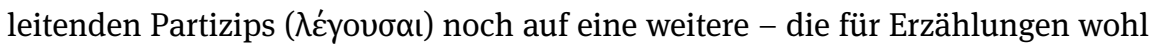
bei Weitem bedeutsamste - Verwendung infiniter Verbformen anstelle von Aussagesätzen mit indikativischer Verbform: das adverbial gebrauchte Partizip.

Zumindest bei temporaler, ${ }^{591}$ modaler ${ }^{592}$ und konzessiver ${ }^{593}$ Sinnrichtung ist es standardmäßig der Fall, dass tatsächlich Realisiertes zum Ausdruck kommt. Offener sind naturgemäß die konditionale ${ }^{594}$ und finale ${ }^{595}$ Sinnrichtung. Beim kausalen Gebrauch wird durch $\dot{\omega} s$ markiert, falls lediglich an einen subjektiven Grund (,in der Meinung, dass ...“ etc.) gedacht ist, ${ }^{596}$ womit der Erzähler zum Ausdruck bringen kann, dass der entsprechende kausale Zusammenhang lediglich in der Vorstellung der Erzählfigur existiert und nicht von ihm geteilt werden muss. ${ }^{597}$ Es handelt sich folglich um ein Signal der Fokalisierung.

Dabei ist vorausgesetzt, dass das Partizip auf ein im Modus des Indikativs formuliertes Verb bezogen ist. ${ }^{598}$ Aber auch dann ist die Gleichwertigkeit mit einem

590 Während das Deutsche ebenso wie wie das klassische Altgriechisch die Äußerung als (lediglich) Wiedergegebenes markieren kann, ist der Konjunktiv nicht notwendig, um einen entsprechenden Effekt zu erzielen: „[B]ei geeigneter expliziter Signalisierung (referatanzeigende Ausdrücke) in abhängigen (daß)-Sätzen [ist] auch der Indikativ mit Nicht-Faktizität und insbesondere Indirektheit vereinbar“ (Zifonun, „Textkonstitutitive Funktionen,“ 325).

591 GGNT 231d.

592 GGNT 231e.

593 GGNT 231g.

594 GGNT 231h.

595 GGNT 231i.

596 GGNT 231f. Vgl. 1. Kor 7,25 und 2. Kor 5,20.

597 Vgl. CGCG 52.39 .

598 CGCG 52.6 merken an: ,the modality (in a broad sense) of a participle is also usually (if not always) relative to the matrix verb.“ Sicherlich nicht ,immer.“ Ein Blick auf 2. Tim 4 verdeutlicht das: In V. 11 heißt es: „Markus mitnehmend, bringe ihn mit dir!“ (Ма̃

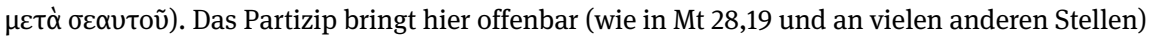
ein Geschehen zum Ausdruck, welches ebenso wie das im Imperativ formulierte Handeln als Teil der auffordernden Sprechhandlung verstanden werden soll. Die Konstellation in V. 13 ist

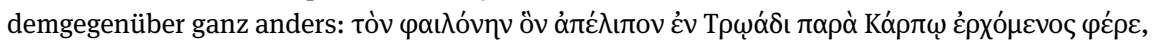

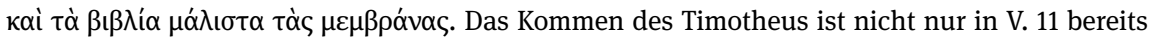

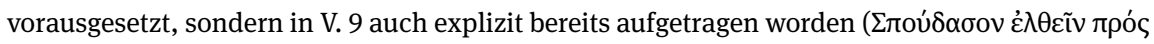
$\mu \varepsilon \tau \alpha \chi \varepsilon \dot{\varepsilon} \omega \varsigma)$. Entsprechend wird das Partizip in V. 11 universal als temporale Umstandsangabe übersetzt, die spezifiziert, wann das im Imperativ aufgegebene Handeln zu erfolgen hat. Das am 
indikativischen Nebensatz abhängig von der lediglich kontextuell erschließbaren gemeinten Sinnrichtung des Partizips: ${ }^{599}$

Ein adverbial gebrauchtes Partizip gibt zunächst (aufgrund seiner grammatischen Funktion) an, unter welchen Umständen, ganz allgemein, der Verbinhalt der übergeordneten Konstruktion verwirklicht wird ... Erst im Einzelkontext lässt sich mit Hilfe der dort operierenden (pragmatischen) Faktoren darauf schließen, welches Segment aus dem möglichen Umständespektrum im Blickfeld steht und in der Übersetzung zum Ausdruck kommen soll. Da es sich bei diesem Spektrum um ein Kontinuum handelt, empfiehlt sich ein eher flexibler Umgang mit den involvierten Kategoriegrenzen.

Wichtig ist, dass in der Koine auch im verneinten Fall kein klares syntaktisches Unterscheidungskriterium vorliegt, da der Gebrauch der Negationspartikel nicht mehr konsequent der Verwendung im analogen Nebensatz folgt, also $\mu$ ' auch dann gebraucht wird, wenn kein Begehren und keine Bedingung ausgedrückt wird. ${ }^{600}$ An einigen Stellen folgt Paulus jedoch dem klassischen Gebrauch, sodass ov̉ an diesen Stellen die Gleichwertigkeit mit einem Behauptungssatz markiert. ${ }^{601}$ Allerdings liegt etwa in der Formulierung 2. Kor 4,8 (,in allem bedrängt, aber

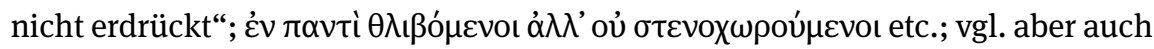
für nicht-klassischen Gebrauch von $\mu$ ' direkt in 4,18) keine eigentliche Erzählung vor, da der zweite Sachverhalt ja gerade negiert ist.

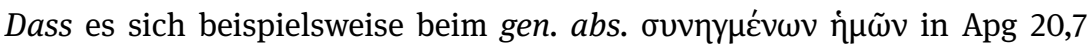
(siehe ausführlicher oben, Kapitel 4, Abschnitt 8.1.1) in Abb. 9 überhaupt um ein adverbiales Partizip mit temporaler Sinnrichtung handelt, muss also erst aus dem Kontext erschlossen werden. Allein auf der Ebene der Grammatik wäre folglich auch nicht ausgeschlossen, dass in Lk 24,23 lediglich das Kommen der Frauen festgehalten wird sowie ihre Intention einen Bericht $\mathrm{zu}$ geben $\left({ }^{\star}\right.$, ,sie

Satzanfang neue eingeführte Thema (vgl. CGCG 60.28; für die Bücher siehe 60.35) fungiert wie in V. 11 (vgl. auch Apg 21,24 etc.) als Akkusativobjekt des rhematischen Imperativs, aber nicht im Hinblick auf das intransitive Partizip. In Mt 2,13 etc. mit derselben Konstellation an Verbtypen

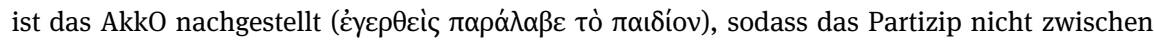

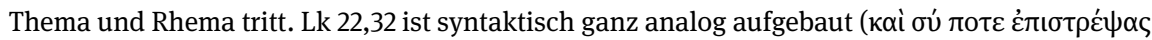

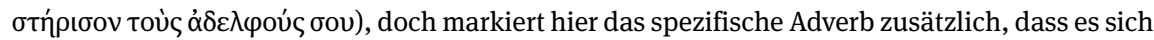
um ein ohnehin irgendwann eintretendes Geschehen handelt - dessen Realisierung dann den Kontext liefert, in welchem das Aufgetragene getan werden soll.

599 GGNT 231c.

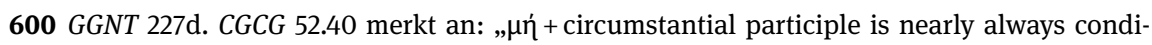
tional.“

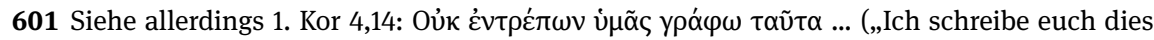
nicht, um euch zu beschämen ...“). 
kamen, um zu sagen ... [Aber sie taten es nicht ...]“"). ${ }^{602}$ Innerhalb der Rahmenerzählung ist aber natürlich explizit festgehalten worden, dass die Frauen das

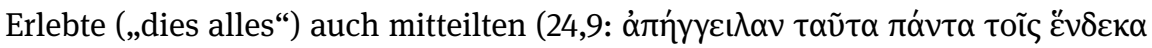

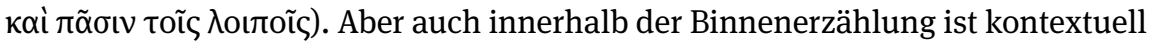
klar davon auszugehen, dass die Frauen nicht lediglich kamen, denn der Vorvers

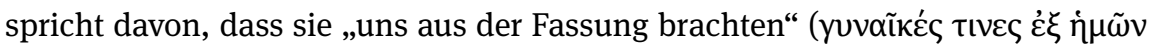

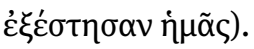

\subsubsection{Durch Verbalnomina aufgegriffene vorerwähnte/bekannte Ereignisse}

Sehr häufig greifen temporale Infinitiv- und Partizipialkonstruktionen in Erzählungen bereits Erzähltes auf, um für ein neues Geschehen auf diese Weise einen temporalen und lokalen Rahmen zu schaffen.

So spricht in Joh 1,48 Jesus zu Nathanael: „Vor dem Rufen ..., unter dem

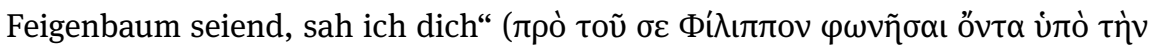

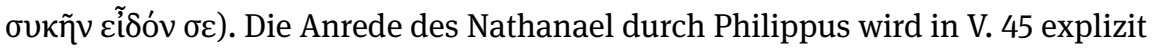
erzählt und in V. 46 als Aufforderung wiedergegeben. Der Verweis auf den Feigenbaum ist demgegenüber überraschend, denn im V. 45 ist nur davon die Rede, dass

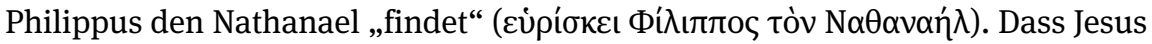
hier auch den Lesern bisher Unbekanntes als temporalen Hintergrund aufgreift, als wäre es bereits bekannt, unterstreicht seine umfassende Kenntnis. ${ }^{603}$

Vergleichbar ist auch Lk 2,21, wo die Namensgebung Jesu indikativisch

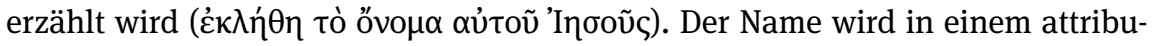
tiven Partizip spezifiziert als „vom Engel genannt“ (

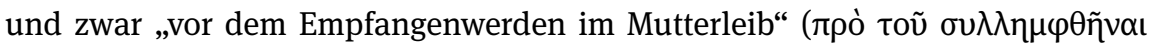

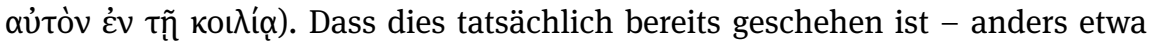

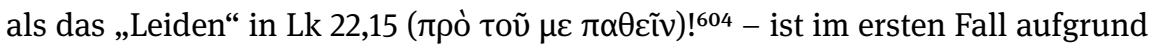
der expliziten Erzählung in Lk 1,31 erschließbar ${ }^{605}$ und im zweiten aufgrund der Reaktion der Elisabeth in Lk 1,42.

In solchen Fällen wird also eigentlich repetitiv erzählt, also mehrfach auf dasselbe Geschehen Bezug genommen. ${ }^{606}$ Dabei handelt es sich bei der erneuten Thematisierung allerdings eher um eine bloße „Erwähnung“ (ob nun mit demselben Verb oder anderen Ausdrücken mit offenbar gleicher Referenz) von bereits

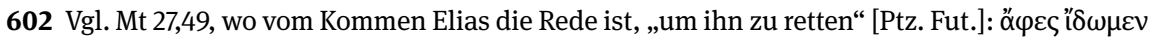

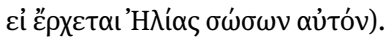

603 Siehe Tripp, ,Jesus’s Special Knowledge“ für die Relevanz für die Erzählung.

604 Ebenso Joh 13,9 und Apg 23,15.

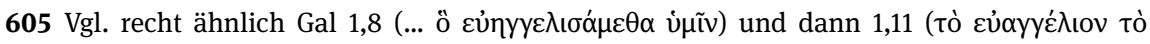

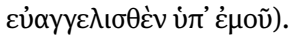

606 Siehe oben, Kapitel 3, Abschnitt 4.2. 
bekannter Information, welche die Kohärenz des Textes unterstützt. ${ }^{607}$ Da durch die indikativisch erfolgte Erzählung der Vergangenheits- (und Wirklichkeits-) Status bereits festgelegt ist, genügt die Nennung des Verbinhalts durch die infinite Form, um eine Verbindung herzustellen.

Relevant für die Analyse sind gerade solche Fälle, in welchen ein Geschehen als „setting“ eines Ereignisses aufgegriffen wird, aber noch nicht erzählt wurde. Das Gemeinte wird etwa anhand von Gal 2,12 deutlich, wo Paulus das Imper-

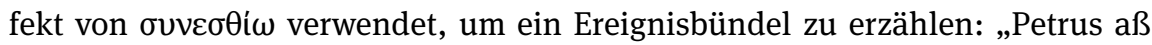
(immer wieder) mit den Heiden“ ( $\mu \varepsilon \tau \dot{\alpha} \tau \tilde{\omega} v \varepsilon \dot{\varepsilon} \theta v \tilde{\omega} v \sigma v v \eta ́ \sigma \theta \varepsilon v)$. Dieser Hauptsatz steht in temporalen Konnexion mit der vorangestellten Infinitiv-Konstruktion

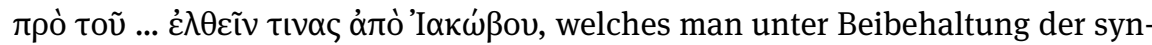
taktischen Besonderheit als „Vor dem Kommen einiger von Jakobus“ ins Deutsche übertragen könnte. Die Infinitivkonstruktion drückt einen Verbinhalt aus, impliziert aber nicht notwendigerweise, dass dieses Ereignis tatsächlich in der Vergangenheit stattgefunden hat. Erst im Fortgang des Textes wird dieses Ereignis auch indikativisch erzählt, das tatsächliche Eintreten des Ereignisses in der Vergangenheit also sprachlich eindeutig zum Ausdruck gebracht: „Als sie aber kamen ..." (ő $\delta \varepsilon \delta \dot{\varepsilon} \tilde{\eta} \lambda \theta o v)$. Auffällig ist in V. 12, dass die infinite Formulierung

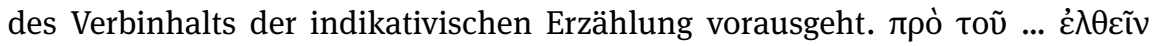
$\tau \iota v \alpha \varsigma$ árò 'I $\alpha \kappa \omega \dot{\beta}$ ov führt hier ein neues „Setting“ ein, wobei Paulus offenbar davon ausgeht, dass die Leser das so angesprochene Geschehen zeitlich verorten können (was dafür spricht, dass sie über das Erzählte bereits andere Erzählungen gehört haben). ${ }^{608}$

\subsection{Zur Pragmatik nicht-indikativischer Satzäquivalente}

Der Gebrauch von Partizipien statt indikativischer Verbformen in Erzählungen bringt einen großen Vorteil mit sich: „Participles make the communication lighter, requiring less processing energy in terms of Relevance Theory. The communication is more streamlined and audiences follow the main points more easily on the first presentation.“609 Zugleich ist diese „condensation of the communication“ auch mit gewissen Abstrichen verbunden: „the relationship between the clauses

607 Vgl. auch GGNT 350-351.

608 Siehe CGCG 60.32 zum Setting aus funktionaler Satzperspektive. Vergleichbar wäre etwa der gen. abs. in Xenophon, Cyr. 4.2.1, welcher jedoch gerade auf im Text bereits Erwähntes zurückverweist.

609 Buth, „Participles,“ 289. 
is no longer specified."610 So wäre in Apg 20,7 (vgl. Abb. 9) beispielsweise ein Nebensatz mit ő $\varepsilon$ syntaktisch aufwändiger, aber zugleich ein deutlicherer Indikator der gemeinten Konnexion. ${ }^{611}$

Es findet also durchaus eine „Hevorhebung“ statt, wenn ein Autor innerhalb der ihm oder ihr für die Realisierung zur Verfügung stehenden Ausdrucksmöglichkeiten eine Realisierung wählt, welche syntaktisch aufwändiger und semantisch gehaltvoller ist. So kommentiert Buth die Wahl eines iv $\alpha$-Satzes anstelle eines Infinitivs wie folgt: „A heavier structure means that the author considered it important to raise the structural importance of the intended event and that the effect was worthwhile even if it required epending more processing energy on the part of the author and the audience." 612

In dieser Arbeit wird davon ausgegangen, dass der Gebrauch von infiniten Satzäquivalenten in Erzählungen als Entlastung der Syntax zu verstehen ist, die durch die Pragmatik des Textes ausgeglichen wird. Als Beispiel ließe sich etwa die konsekutive Konnexion in 2. Kor 7,9 anführen. Die Geschehens-Proposition ist hier

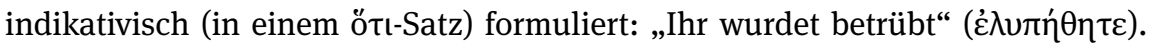
Die RESULTAT-Proposition wird lediglich durch die Formulierung „zur Buße“ (عiৎ

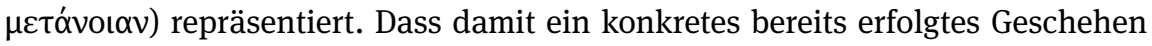
gemeint ist, ergibt sich aus dem Kontext und ist den Erstlesern natürlich auch so bewusst. Entsprechend übersetzt etwa die NGÜ indikativisch, dass der Schmerz „zur Umkehr gebracht hat.“

\subsection{Fazit: Konsequenzen für die Analyse}

Zunächst muss festgehalten werden, dass eine Proposition durchaus nicht durch einen Hauptsatz mit indikativischem Verb zum Ausdruck gebracht werden muss. Auch Nebensätze und „satzwertige Konstruktionen“ mit Infinitiv oder Partizip und selbst Verbalsubstantive nach Präpositionen konstituieren Propositionen. ${ }^{613}$

Entsprechend können sämtliche Konnexionen, die auf der Koordination oder Subordination von indikativischen Sätzen durch Konjunktionen beruhen, auch durch nicht-finite Konstruktionen umformuliert werden, ohne dass dies die Inhalts-Seite der Textstruktur beeinflussen müsste. Für das hierarchische Propositionalgefüge eines Textes ist es nicht notwendigerweise bedeutsam, ob

610 Buth, „Participles,“ 289. Vgl. im selben Band ähnlich auch Runge, „Grounding Status,“ 264. 611 Vgl. zu den Möglichkeiten GGNT 330a.

612 Buth, „Participles,“ 279.

613 Vgl. GGNT 312a. 
die grammatische Kohärenz durch finite oder infinite Verbformen hergestellt wird.

Die von Heinrich von Siebenthal zu den jeweiligen Konnexionen zusammengestellten Übersichten ${ }^{614}$ geben einen Einblick in die Vielzahl der sprachlichen Ausdrucksmöglichkeiten der jeweiligen Zusammenhänge. Das Griechische weist hier eine viel größere Bandbreite sprachlicher Ausdrucksformen auf als das Deutsche, wobei man natürlich auch die von Köppe und Kindt gelieferten Beispiele von minimalen Erzählungen temporal geordneter und sinnhaft verbundener Ereignisse in weniger übliche Konstruktionen umformulieren könnte, wie Abb. 50 zeigt.

\begin{tabular}{|l|l|l|}
\hline Indikativischer Normalfall & $\begin{array}{l}\text { Erst hing der Apfel am } \\
\text { Baum. } 615 \\
\text { Und dann fiel er herunter. }\end{array}$ & $\begin{array}{l}\text { Während die Polizei noch } \\
\text { nach ihm fahndete, } \\
\text { setzte sich Peter ins Ausland } \\
\text { ab. }\end{array}$ \\
\hline $\begin{array}{l}\text { Mit substantiviertem } \\
\text { Infinitiv }\end{array}$ & $\begin{array}{l}\text { Nach dem Hängen am Baum } \\
\text { fiel der Apfel herunter. }\end{array}$ & $\begin{array}{l}\text { Während des noch } \\
\text { anhaltenden Fahndens der } \\
\text { Polizei nach Peter } \\
\text { setzte sich dieser ins Ausland } \\
\text { ab. }\end{array}$ \\
\hline $\begin{array}{l}\text { Mit satzwertiger } \\
\text { Partizipialphrase } 616\end{array}$ & $\begin{array}{l}\text { Am Baum gehangen, } \\
\text { fiel der Apfel herunter. }\end{array}$ & $\begin{array}{l}\text { Immer noch von der Polizei } \\
\text { gesucht, } \\
\text { setzte sich Peter ins Ausland } \\
\text { ab. }\end{array}$ \\
\hline $\begin{array}{l}\text { Mit Aktionsnomen } \\
\text { hor seinem Sturz }\end{array}$ & $\begin{array}{l}\text { Während der noch } \\
\text { anhaltenden Fahndung der } \\
\text { Polizei nach Peter } \\
\text { setzte sich dieser ins Ausland } \\
\text { ab. }\end{array}$ \\
\hline
\end{tabular}

Abb. 50: Variation der Beispielserzählung von Köppe und Kindt.

614 GGNT 325-345. Vgl. die alphabetische Übersicht bei GGNT 354.

615 Zur Markierung der Hauptsätze wurde hier gegenüber Köppe und Kindt, Erzähltheorie, 50 ein Punkt eingefügt.

616 Köppe und Kindt, Erzähltheorie, 65 nutzen diese Ausdrucksmöglichkeit in ihrem Versuch, die Ereignisse der Erzählung in Beziehung zum mentalen Innenleben des Apfels in Beziehung zu setzen: „Erst hielt sich der Apfel noch am Baum, dann stürzte er, von seinen Kräften verlas- 
Auch wenn auf der Inhalts-Seite des Textes das Propositionalgefüge identisch ist, bedeutet dies allerdings nicht, dass die Varianz auf der Grammatik-Seite des Textes kommunikativ irrelevant wäre. Die Wahl nicht-indikativischer Verbformen in Erzählungen bieten dabei vor allem die Möglichkeit, Rückschlüsse über die pragmatische Situiertheit des Textes anzustellen (vgl. für Details den vorangehenden Abschnitt 7.3). Es bietet sich daher an, narrative Vertextungsmuster nicht nur im Hinblick auf ihre Propositionalstruktur hin zu untersuchen, sondern auch zu beachten, inwiefern die Verortung der erzählten Ereignisse in der tatsächlichen (erzählten) Vergangenheit grammatisch festgelegt ist und wo sich diese Aspekte lediglich aus dem kommunikativen Kontext erschließen lassen.

An dieser Stelle wäre es durchaus möglich, ein prototypisches Verständnis der grammatischen Textstrukturseite von Erzählungen zu erwägen: Eine Erzählung, welche die minimalistische Definition erfüllt, würde dann im Normalfall in einem solchen Sinn von Ereignissen ,handeln,“ dass diese durch indikativische Verben zum Ausdruck gebracht würden. Läge - abweichend von diesem Normalfall - eine nicht-indikativische Realisierung vor, so müsste man diesen Spezialfall aus dem konkreten Kommunikations-Kontext heraus erklären.

Allerdings dürfte es bei tatsächlich geschehenden Erzählakten gerade der Standardfall sein, dass immer auch bereits eine (wie auch immer geartete) zuvor bestehende Beziehung der beteiligten Parteien vorausgesetzt werden kann, sodass eigentlich beim Vollzug des Erzählens immer mit (unterschiedlich weit gehender) „pragmatischer Entlastung“ zu rechnen ist. Dazu kommt noch der durchaus gängige Bezug auf im Text bereits Erwähntes. Der erwogene Prototyp dürfte daher kaum in Reinform realisiert vorliegen.

Es bietet sich daher ein alternatives definitorisches Vorgehen an: Ebenso wie man die Menge der die Minimalbedingungen erfüllenden Erzählungen durch das Hinzufügen pragmatischer Bedingungen weiter eingrenzen kann, um ein gehaltvolleres Verständnis der Kategorie ,Erzählung‘ zu entwickeln (so Köppe und Kindt), ${ }^{617}$ lässt sich auch eine weitere (eine andere Teilmenge bildende) Eingrenzung auf syntaktischer Ebene vornehmen, indem man die sprachliche Realisierung der Ereignisbezüge auf Indikativ-Verbformen beschränkt.

Ein Text wäre dann nur dann als ,Erzählung، in diesem speziellen Sinn zu bezeichnen, wenn er ausschließlich ${ }^{618}$ aus Propositionen bestünde, deren Kerne

sen, ab.“ Die Autoren fügen damit ein zusätzliches Ereignis ein, das in kausaler Beziehung zum „Sturz“ steht. Entsprechend könnte man die satzwertige Phrase auch umformulieren (siehe Duden 1326): „Da den Apfel die Kräfte verlassen hatten, stürzte er ab.“

617 Vgl. aber auch oben, Kapitel 3, Abschnitt 3.4.5 für eine kritische Anfrage.

618 Die Definition von Köppe und Kindt lässt zu, dass ein Text auch sprachliche Elemente enthält, die nicht von Ereignissen handeln. Denn einerseits erfüllt eine Erzählung im minimalen 
indikativisch ausgedrückt wären. Um Verwechslungen mit der ebenfalls eine geringere Menge umfassenden Kategorie der Erzählung im gehaltvolleren (d.h. pragmatisch signifikanteren) Sinn nach Köppe und Kindt zu vermeiden, wird diese Kategorie im Folgenden als „strikte“ Erzählung bezeichnet. Es handelt sich dabei nicht um eine Erzählung, welche die Mindestbedingungen von Narrativität erfüllt (wie dies auch für Miniaturerzählungen gilt, sodass die Menge hier größer ist als im Fall der gehaltvolleren Definition), sondern eher um eine „minimalistische“ Erzählung im Sinne eines Textes, der keinerlei Elemente aufweist, bei welchen unter pragmatischen Gesichtspunkten die Verbgrammatik entlastet wird.

Das Verhältnis der strikten Erzählung zu den beiden verschieden eng gefassten Konzeptionen von Erzählung bei Köppe und Kindt ist ähnlich, wie die Beziehung, welche diese Autoren zwischen einer Erzählung im gehaltvolleren Sinn und einem (komplexen, literarischen) „Erzählwerk“ sehen: Ein Roman als Ganzer stellt einen Text dar, in welchem die definitorischen Eigenschaften der verschiedenen Erzählungs-Konzepte mehr oder weniger durchgehend umgesetzt sind. ${ }^{619}$ Eine „konzise Erzählung“ bildet dann in der Regel erst der Leser aus dem Stoff des Romans. ${ }^{620}$ Ebenso kann man sagen: Das Muster ${ }^{621}$ der strikten Erzählung wird in Texten, welche Köppe und Kindt als (minimale oder gehaltvollere) Erzählungen auffassen, in unterschiedlichem Umfang - d. h. mit unterschiedlicher Durchgängigkeit - umgesetzt.

Sinn zwar per Definition die Bedingung der Textualität (Köppe und Kindt, Erzähltheorie, 44), anders herum wird aber nicht gefordert, dass der als Erzählung klassifizierte Text nicht auch anderes als Ereignisrepräsentationen enthalten dürfte.

619 Köppe und Kindt, Erzähltheorie, 101.

620 Köppe und Kindt, Erzähltheorie, 101-102.

621 Vgl. Köppe und Kindt, Erzähltheorie, 101. 